\title{
Lipid-Nanodiscs Formed by Paramagnetic Polymers for Fast NMR Data Acquisition
}

\author{
by
}

\author{
Giacomo M. Di Mauro \\ A dissertation submitted in partial fulfillment \\ of the requirements for the degree of \\ Doctor of Philosophy \\ (Chemistry) \\ in The University of Michigan \\ 2021
}

Doctoral Committee:

Professor Ayyalusamy Ramamoorthy, Chair

Professor Zhan Chen

Professor Jingsang Kim

Professor Kenichi Kuroda 
Giacomo M. Di Mauro

gdimauro@umich.edu

ORCID iD: 0000-0002-6990-1304

(C) Giacomo M. Di Mauro 


\section{DEDICATION}

To my beloved wife, Debora, whose love and care supported me during this journey. 


\section{ACKNOWLEDGEMENTS}

During these four years, besides the research shown in this thesis, I have been involved in many extra-curricular activities. The culturally vibrant environment at the University of Michigan made me interact with many outstanding scholars and spectacular people. Either for a coffee break (one of the many), a lecture, a seminar, or any other formal and informal academic interactions, I received so much warmth and support that writing this section is quite challenging. My goal is to dedicate a special thanks to all of them.

First of all, I want to express my gratitude to my former research advisor and friend, professor Carmelo La Rosa, without whom I could not have been where I am. Thanks to his past and current research group at the University of Catania for all the adventures lived together.

I am thankful to my current research advisor, Prof. Ayyalusamy Ramamoorthy, who, throughout these years, taught me invaluable lessons that I will never forget.

Thanks to my committee members Prof. Zhan Chen, Prof. Jinsang Kim, and Prof. Kenichi Kuroda, for the crucial support and scientific comments in the present work. Thank you for preparing me for a successful career.

I am also thankful to all the scholars that passed from the Ramamoorthy lab. Together we created great science and shared fun moments dining together and sipping coffee (a lot!). Thus, thank you to all my lab mates, past and present, that made these years enjoyable in the lab. I learned a lot from you all.

If I am writing these notes today, this could not have happened without the Chemistry Department's support at the University of Michigan and its brilliant community. Being an international student is always challenging. Still, everyone has been incredibly supportive, contributed to my success and well-being. However, a special mention goes to the Doctoral Program Coordinator, Ms. Katie Foster, for the irreplaceable support. Moreover, being in the Ramamoorthy lab also made me part of the Biophysics department, populated by terrific 
researchers and amazing people. I want to thank them all for welcoming me into their "family" and for the incredible support and friendship.

I am immensely thankful to all my students, "victims" of my growing teaching skills, and all the GSIs with whom I shared valuable teaching tips and exciting grading moments.

Thanks to Prof. Alexander Poniatowski, my mentor in the Future Faculty GSI program, and all the professors and lecturers for the mentorship that I received since my early appointments as an instructor.

Special mention goes to the fantastic organizing committee of the Karle Symposium 2020. This event's organization required an incredible dose of adaptability toward a fluid situation since we had to face unexpected challenges due to a global pandemic. Thanks to the co-chair, Zachary Fejedelem, for sharing the burden with me. Thanks to the whole team and stakeholders for their dedication and support, and the faculty advisors Prof. Paul Zimmerman and Dr. Yulia Sevryugina, for their invaluable help. The event could not have taken place without the effort of all of them. A special thanks also go to the fantastic organizing committees of ALUM|NUM, and URAN|UM.

I want to express my gratitude to Chris Chiaravalli, Emilia Giralt, Francesca Terzoli, Joseph Jafri, all my friends from the Italian Student Association (ITSA), and all the fantastic students I met during seminars and conversation hours.

Thanks to all my friends from the "Science and Beer group" and the post-doc association. You have all been terrific friends and adventure buddies. You have also been real mentors and a proper anchor to maintain my mental health during the most challenging days of my Ph.D. adventure.

Thanks to my family and all my friends that supported me from Italy. I felt your love despite the distance. A wholehearted thanks go to Livio Sgarlata for his crucial support in making 3D models for many figures shown in my thesis.

Last but not least, thank you, Debora Di Pietro, for being the most beautiful, caring, and supporting wife. Without your unconditional love and support, I certainly would not have made it.

I am looking forward to starting my new adventure. Ad Maiora.

Giacomo M. Di Mauro, Ann Arbor, 15 December 2020 


\section{TABLE OF CONTENTS}

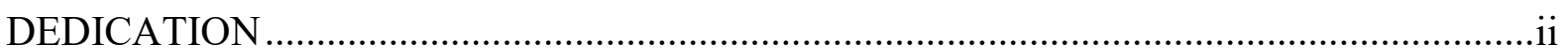

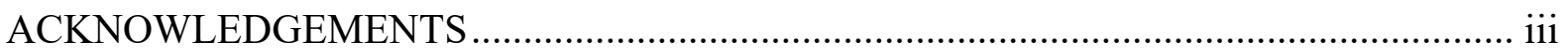

LIST OF ABBREVIATIONS ................................................................................... viii

LIST OF FIGURES …....................................................................................... xiii

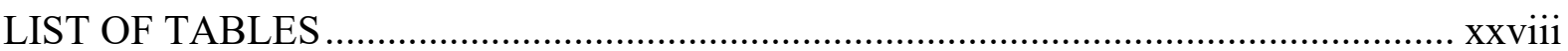

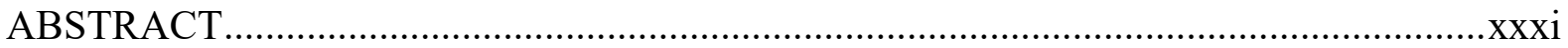

Chapter 1 Introducing SMALP for the Study of Membrane Proteins .................................... 1

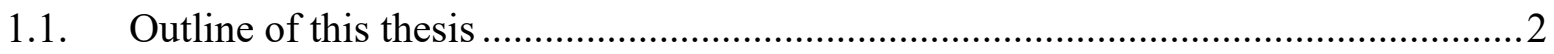

1.2 Biological membranes and membrane mimetics ...................................................5

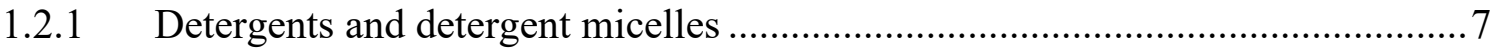

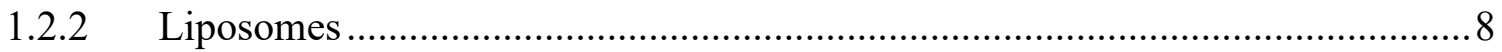

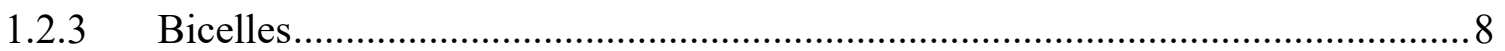

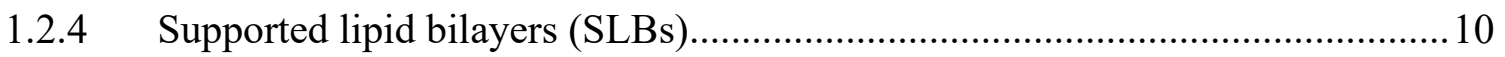

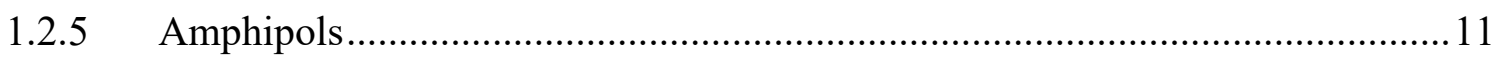

1.2.6 MSP-Nanodiscs, Salipro, peptidiscs, and copolymer-nanodiscs ....................... 12

1.2.7 Styrene-maleic acid lipoparticles and copolymer-nanodiscs ............................ 14

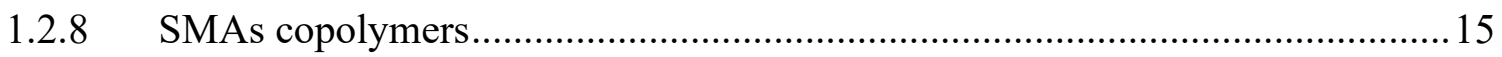

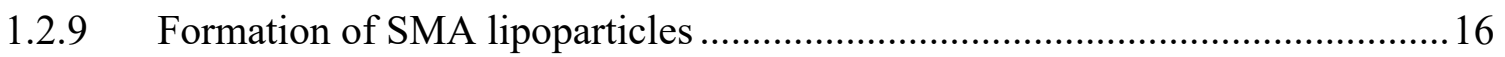

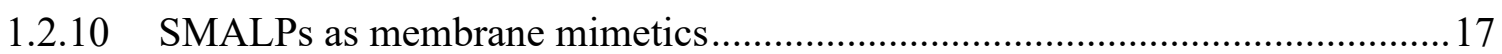

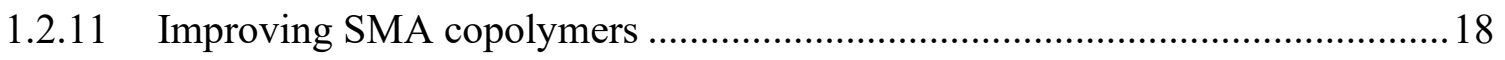

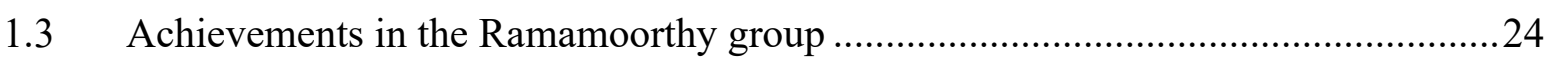

1.3.1 Modified SMALPs as membrane mimicking systems.....................................24 
1.3.2 Expanding the range of sizes nanodiscs by using SMA-EA.............................25

1.3.3 Acidic compatible polymer nanodiscs using SMA-ED and SMAd-A ..............26

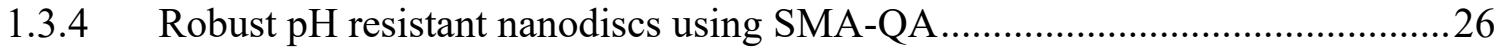

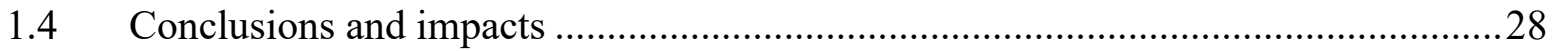

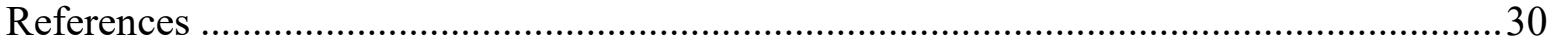

Chapter 2 Use of Paramagnetic Systems to Speed-up NMR Data Acquisition and for Structural

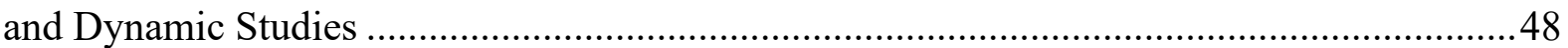

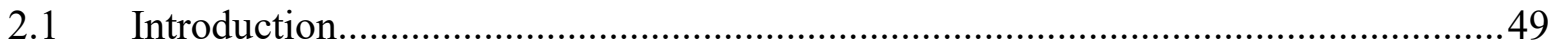

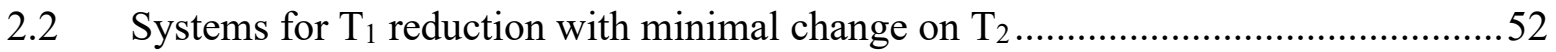

2.3 PRE molecules can be used to obtain structural information ..................................54

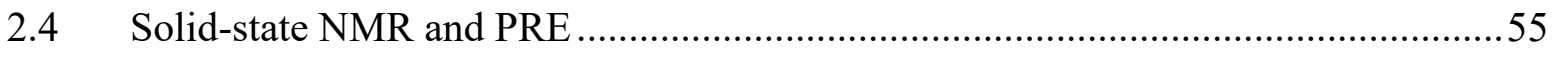

2.5 Biological membranes: bicelles and nanodiscs ..................................................57

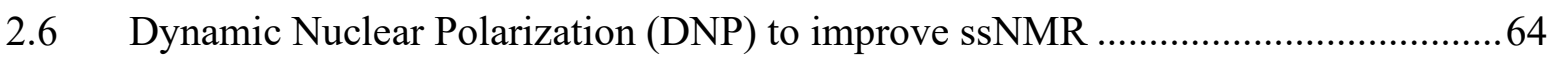

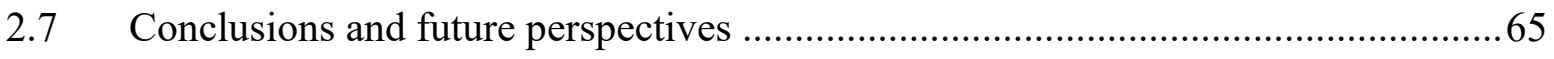

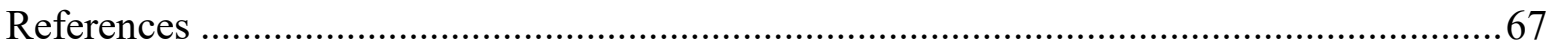

Chapter 3 Lipid-Nanodiscs Formed by Paramagnetic Metal Chelated Polymer for Fast NMR

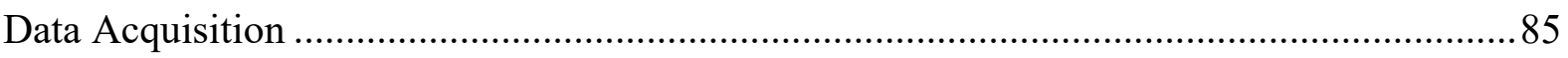

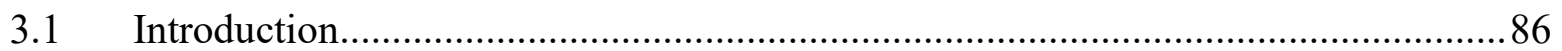

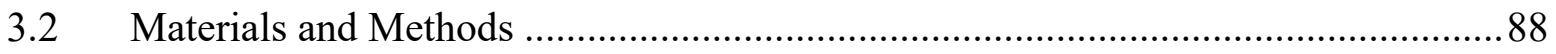

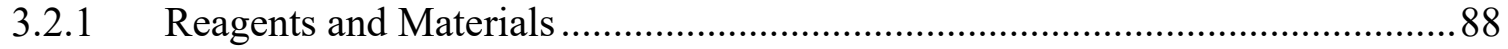

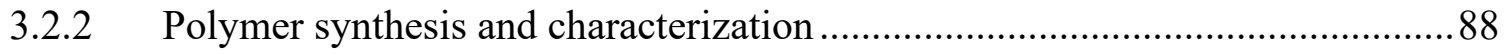

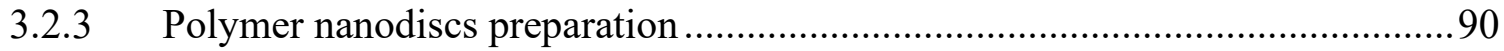

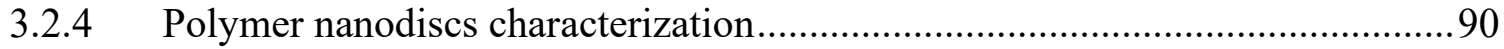

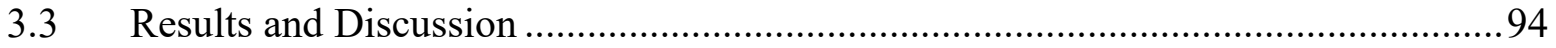

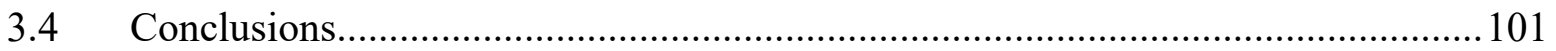

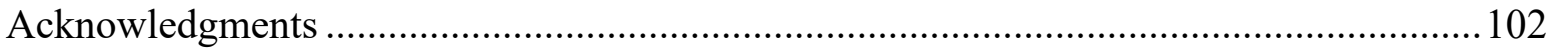

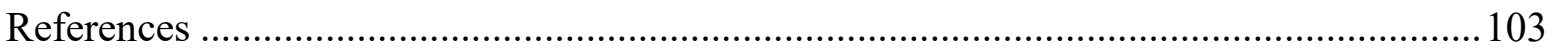


Chapter 4 Benchmarks of SMA-Copolymer Derivatives and Nanodiscs Integrity

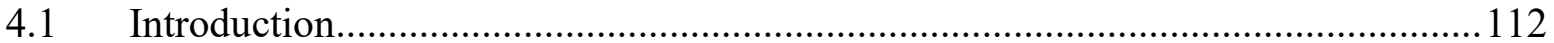

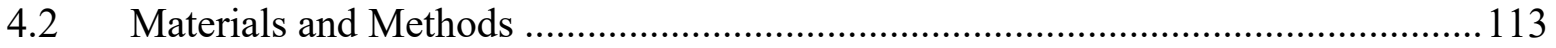

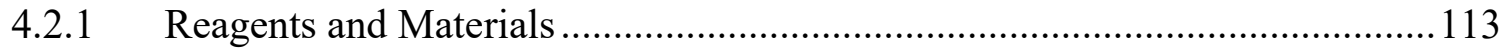

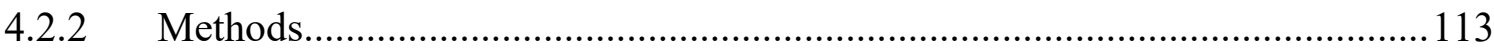

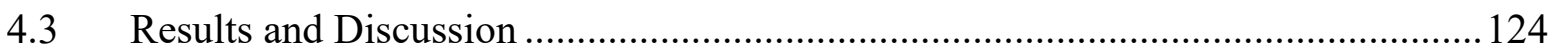

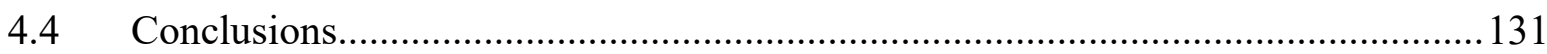

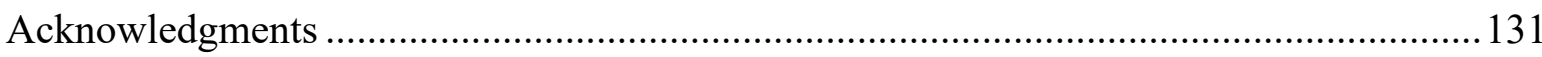

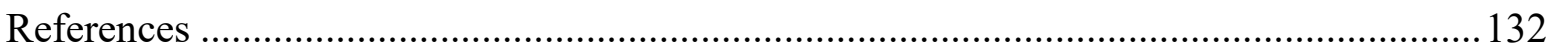

Chapter 5 Spin-Labeled Copolymer Nanodiscs for DNP Studies on Membrane Proteins .... 139

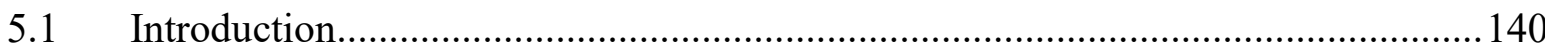

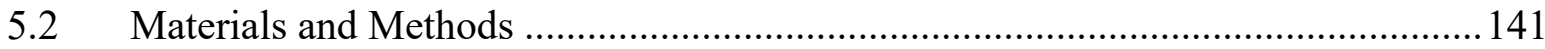

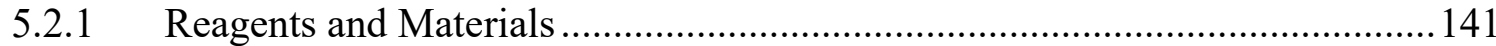

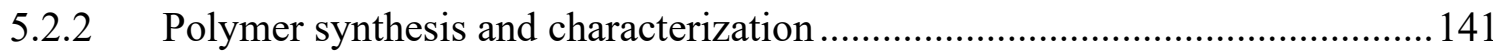

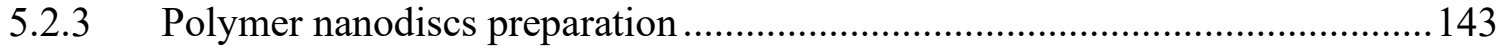

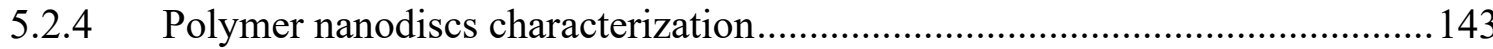

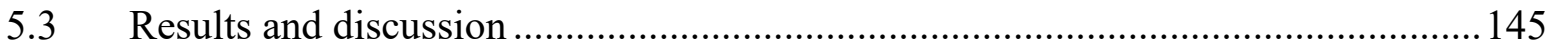

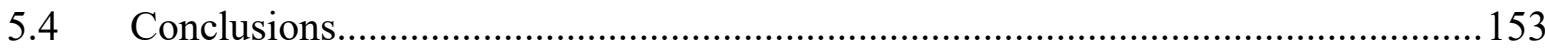

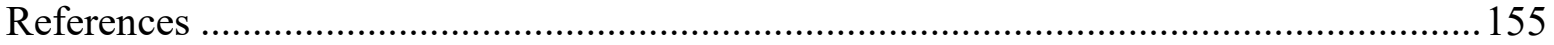

Chapter 6 Future Directions and Conclusions. Alternative Uses of Copolymer-Based

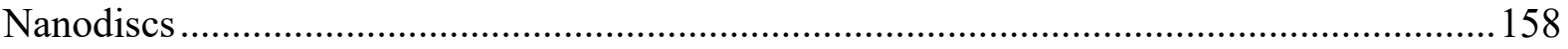

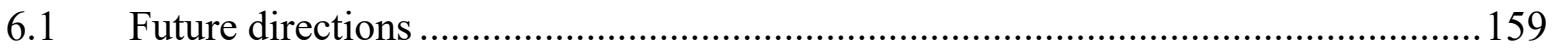

6.1.1 Polymer-nanodiscs in nanomedicine ......................................................... 159

6.1.2 Magnetic resonance imaging (MRI) and contrast agents ............................. 162

6.1.3 Nanodiscs as valid biomimetic MRI contrast agents ................................... 164

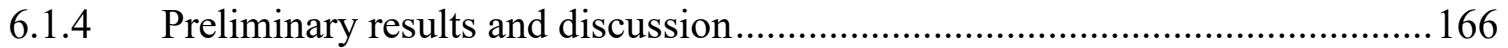

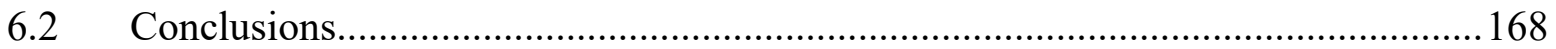

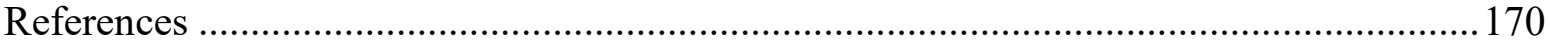

Appendix 179 


\section{LIST OF ABBREVIATIONS}

\section{Abbreviation Extended}

\begin{tabular}{ll}
\hline AFM & Atomic Force Microscopy \\
APols & Amphipols \\
$\begin{array}{l}\text { Boc (protecting } \\
\text { group) }\end{array}$ & Tert-butyloxycarbonyl (protecting group) \\
CA & Contrast Agent \\
CD & Circular Dichroism \\
CEST & Chemical Exchange Saturation Transfer \\
CG modeling & Coarse-grain modeling \\
c.m.c. & Critical Micelle Concentration \\
Cryo-EM & Cryogenic Electron Microscopy \\
CSA & Chemical Shift Anisotropy \\
CSTR & Continuous Stirred-Tank Reactor \\
Cyt b5 & Cytochrome b5 \\
CW-EPR & Continuous Wave-EPR (spectroscopy) \\
DIBMA & Diisobutylene Maleic Acid copolymer \\
DLS & Dynamic Light Scattering \\
DMPC & 1,2-dimyristoyl-sn-glycero-3-phosphocholine (14:0 PC) \\
DMPE-DTPA & \\
& \\
&
\end{tabular}


DNP

$\mathrm{DO} 2 \mathrm{~A}$

DOPC

DOTA

DPPC

DSPC

DTPA

EDTA

EPR (spectroscopy)

EPR (effect)

ePSMA

FDA

FID

FRAP

FT-IR

GBCA

GUV

HEPES

HETCOR

HDL

HMQC

HSQC

LUV

LTO

MAS

MD

MLV
Dynamic Nuclear Polarization

1,7-dicarboxymethyl-1,4,7,10-tetraazacyclododecane

1,2,-dioleyl-sn-glycero-3-phosphocholine [18:1 ( $\Delta^{9}$-Cis)PC]

1,4,7,10-tetraazacyclododecane-1,4,7,10-tetraacetic acid

1,2-dipalmitoyl-sn-glycero-3-phosphocholine (16:0 PC)

1,2-distearoyl-sn-glycero-3-phoshocholine (18:0 PC)

Diethylenetriaminepentaacetic acid

Ethylenediaminetetraacetic acid

Electron Paramagnetic Resonance (spectroscopy)

Enhanced Permeability and Retention effect

Poly(styrene-co-maleic acid) - Methyl esther

Food and Drug Administration (US Federal Agency)

Free Induction Decay

Fluorescence Recovery After Photobleaching (spectroscopy)

Fourier Transform Infrared (spectroscopy)

Gadolinium-based Contrast Agent

Giant Unilamellar Vesicle

4-(2-Hydroxyethyl)Piperazine-1-ethanesulfonic acid

Heteronuclear Correlation

High-Density Lipoparticle

Heteronuclear Multiple Quantum Coherence

Heteronuclear Single Quantum Coherence

Large Unilamellar Vesicle

Lithium-Titanium-Oxide Spinel

Magic Angle Spinning

Molecular Dynamics (simulations)

Multilamellar Vesicle 
Membrane Protein

MRI

Magnetic Resonance Imaging

MSP

Membrane Scaffold Protein

MTSL

(S-(1-oxyl-2,2,5,5-tetramethyl-2,5-dihydro-1H-pyrrol-3-yl)methyl methanesulfonothioate)

MW

Molecular Weight

NCS

Neocarzinostatin

NMP

N-Methyl-2-pyrrolidone

NMR

Nuclear Magnetic Resonance (spectroscopy)

NOTA

1,4,7-Triazacyclononane-1,4,7-triacetic acid

NSF

Nephrogenic Systemic Fibrosis

PAA

Poly(acrylic acid)

PARACEST

Paramagnetic Chemical Exchange Saturation Transfer

PCS

Pseudo-contact Shift

PCT

Proton Tomography Imaging

PDB

Protein Data Bank

PET

Positron Emission Tomography

PISEMA

Polarization Inversion Spin Exchange at the Magic Angle

PMA

Poly(methyl acrylate)

POPC

1-palmitoyl-2-oleoyl-glycero-3-phosphocholine (16:0-18:1 PC)

POPG

1-palmitoyl-2-oleoyl-glycero-3-phospho-(1'-rac-glycerol) (16:018:1)

PRE

Paramagnetic Resonance Enhancement

RDC

Residual Dipolar Coupling

RAFT

Reversible Addition-Fragmentation Chain-Transfer

Polymerization

RF

Radio Frequency

RFDR

Radio Frequency-driven Dipolar Recoupling

SANS

Small Angle Neutrons Scattering 
SAXS

SEC

SLB

SLF

SLN

SLS

SMA

SMAd-A

SMAs

SMA-EA

SMA-EA-DOTA

SMA-ED

SMALP

SMAnh

SMA-Glu

SMA-Neut

SMA-Pos

SMA-QA

SMA-SH

SMANCS

SMI

SOFAST-HMQC SSNMR

ST-10

ST-100

ST-X

STD
Small Angle X-Rays Scattering

Size-Exclusion Chromatography

Supported Lipid Bilayer

Separated Local Field

Sarcolipin

Static Light Scattering

Poly(styrene-co-maleic acid)

Poly(styrene-co-maleimide) - Amine

SMA polymer family

Poly(styrene-co-maleic acid) - Ethanolamine

Poly(styrene-co-maleic acid) - Ethanolamine - $(1,4,7,10$ tetraazacyclododecane-1,4,7,10-tetraacetic acid)

Poly(styrene-co-maleic acid) - Ethylene diamine

Poly(styrene-co-maleic acid) lipoparticles

Poly(styrene-co-maleic anhydride)

Poly(styrene-co-maleic acid) - Glucose

Poly(styrene-co-maleic acid) - Neutral

Poly(styrene-co-maleic acid) - Positively charged

Poly(styrene-co-maleimide) - Quaternary ammonium

Poly(styrene-co-maleic acid) - Aminoethanthiol

Poly(styrene-co-maleic acid) - Neocarzinostatin

Poly(styrene-co-maleimide)

Band-Selective Optimized Flip Angle Short Transient HMQC

Solid-State NMR

Poly(styrene-co-maleic acid) - 10-15\% TEMPO

Poly(styrene-co-maleic acid) - 100\% TEMPO

Poly(styrene-co-maleic acid) - X\% TEMPO

Saturated-Transfer Difference 
STMA

SUV

$\mathrm{T}_{1}$

$\mathrm{T}_{2}$

TAHA

TEM

TEMPO

TIRF

TFA

ThT

TROSY-HSQC

XIRAN®

zSMA
Poly(stilbene-co-maleic acid)

Single Unilamellar Vesicle

Spin-lattice relaxation time

Spin-spin relaxation time

Triaminohexaacetate

Transmission Electron Microscopy

(2,2,6,6-tetramethylpiperidin-1-yl)oxyl

Total Internal Reflection Fluorescence (microscopy)

Trifluoro acetic acid

Thioflavin-T

Transverse Relaxation-Optimized Spectroscopy HSQC

SMA formulations by Polyscience ${ }^{\circledR}$ (Geleen, Netherlands)

Zwitterionic SMA 


\section{LIST OF FIGURES}

\section{Chapter 1}

Figure 1.1. The cell membrane is a fluid mosaic model. The phospholipid bilayer comprises many different molecular components, including a variety of lipids, proteins, and cholesterol, some of which are with carbohydrate groups attached. This "fluid lipid sea" hosts other molecules such as membrane proteins ${ }^{1,2,13}$. This illustration is licensed under the Creative Commons Attribution 4.0 International ${ }^{14}$

Figure 1.2. Classification of cell membrane mimetics. On the left non-bilayer model systems such as detergent micelles, while on the right are lipid bilayer model systems such as liposomes, SLBs, bicelles, and nanodiscs

Figure 1.3. Molecular structures of widely employed detergents in the extraction of membrane proteins. Detergents are classified as ionic (either negatively or positively charged), zwitterionic, and non-ionic. A few examples of commonly used molecular systems for each of the categories are given.

Figure 1.4. Supramolecular architecture of micelles, bicelles, and liposomes. The figure shows the commonly used membrane mimicking systems. For example, detergent or lipid micelles and isotropic bicelles that tumble sufficiently fast in the NMR time scale are frequently used in solution NMR studies whereas the large-size membrane mimetics such as large-size bicelles and liposomes/vesicles are commonly used in solid-state NMR applications. While all of these membrane mimetics are commonly used in the structural studies of membrane-associated peptides (including antimicrobial peptides, toxins, fusion peptides, amyloid peptides) and proteins, the lack of non-bilayer structure and curvature of micelles are capable of inducing undesirable effects. We recommend some of the review articles that comprehensively cover all aspects of these mimetics to the readers ${ }^{39,45,49-52}$. 9 
Figure 1.5. Supported Lipid Bilayer (SLB) design and mechanics. (A) SLBs contain a bilayer separated from a rigid substrate by a thin layer of water. (B) Representative FRAP of labeled lipids illustrating SLB lateral fluidity. Lipids in SLBs freely diffuse within the plane on three representative substrates. Following photobleaching, diffusion causes photobleached lipids to be diluted, and the average fluorescence to increase. The disappearance of a visible bleached region indicates total recovery and a fluid bilayer. (C) SLB stiffness in comparison to tissue, hydrogels, and glass substrates. SLBs are anisotropic, behaving like fluids in the XYplane, but stiffer than hydrogels in the Z-direction. The figure is used with permission from ${ }^{54}$. Copyright 2017 Elsevier.

Figure 1.6. Chemical structures of four types of amphipols. (a) A polyacrylate-based APol, A8-35 (87); (b) a phosphorylcholine-based APol, C22-43 (26); (c) a nonionic, glucose-based APol (71); and (d) a sulfonated APol (23). The figure was used with permission from ${ }^{61}$. Copyright 2011 Annual Reviews.

Figure 1.7. Schematic representation of MSP-Nanodiscs. Nanodiscs are discoidal lipid bilayer stabilized by encircling amphipathic helical scaffold proteins termed as membrane scaffold protein (MSP). Reproduced with permission from ${ }^{52}$. Copyright 2014 CRC Press.... 12 Figure 1.8. SMALP: a growing community. a) shows the number of peer-reviewed publications per year since the first publication on SMALP appeared in May 2009. b) shows the type of publication. Since the first article, 8 patents have been deposited, 24 communications and 210 research articles have been published. Also, 46 students received their $\mathrm{Ph} . \mathrm{D}$. with dissertations on a topic that uses SMALP as a tool to investigate membrane proteins. Notably, due to the multidisciplinary nature of the field, there have been 57 review articles in the span of 10 years. Both graphs are updated until 31 December 2020. More details are available at http://www.smalp.net.

Figure 1.9. SMAs' most common formulations. SMA has been used in the styrene-to-maleic acid ratio that goes from 1:1 to 3:1 ratio of styrene:maleic acid. The advantages over micelles, liposomes, bicelles, and protein-based nanodiscs allow polymer nanodiscs to be applied to a broader variety of membrane protein studies.

Figure 1.10. An illustration of a three-step model for the formation of SMA based lipidnanodiscs. Step 1. Electrostatic interactions between the surface of the lipid bilayer and the copolymer chains. Step 2. Insertion of the styrene moieties in the hydrophobic core of the lipid bilayer, driven by the hydrophobic effect. Step 3. The destabilized bilayer evolves into nanodiscs because of the electrostatic repulsion of the hydrophilic maleic acid moieties of the copolymer. 16 
Figure 1.11. Representative differential scanning calorimetry (DSC) thermograms of di16:0 PC lipids self-assembled in MLVs, SMA 2:1 nanodiscs and SMA 3:1 nanodiscs (left panel). Nanodiscs were obtained at a SMA-to-lipid mass ratio of $\sim 1.7$. The inserted scale bar at the top (MLVs) corresponds to $5 \mathrm{kcal} / \mathrm{mol}{ }^{\circ} \mathrm{C}$ while the bar at the bottom (nanodiscs) corresponds to $0.5 \mathrm{kcal} / \mathrm{mol}^{\circ} \mathrm{C}$. (Right panel) Variation in A) $\mathrm{T}_{\mathrm{m}}$ and B) $\Delta \mathrm{H}_{\text {cal }}$ of the gel-tofluid phase transition of di-16:0 PC lipids in nanodiscs bounded by SMA 2:1 (blue circles) SMA 3:1 (green circles) or SMA 4:1 (red circles). Data are given as averages obtained from the 2 nd and 3rd heating cycle from 2 independent samples. Errors representing the standard deviation are covered by the markers. The figure is reproduced with permission from ${ }^{133}$. Copyright 2017 Elsevier.

Figure 1.12. Dispersity of polymers. Regardless of their composition, synthetic polymers are dispersed. Dispersity, defined as $Đ=M_{w} / M_{n}$, shows how the term "polymer" refers to a plethora of macromolecules with different molecular weights distribution. c) denotes the ideal case, typical of small molecules. b) refers to narrow molecular weight distribution, while a) simulates the actual synthetic polymers. The larger the value of $Đ$ is, the more disperse is the polymer sample.

Figure 1.13. Variants of functionalization of the copolymeric backbone. The maleic acid moiety can be "substituted" by maleimide rings (a) (e.g., SMI ${ }^{152}$, SMA-QA ${ }^{153}$ ) or functionalized with pending groups (b) achieving different charge sign and densities (e.g., zSMA $^{154}$, SMAd-A ${ }^{155}$, SMA-EA ${ }^{156}, \mathrm{SMA} \mathrm{ED}^{155}, \mathrm{SMA} \mathrm{SH}^{157}$, etc.). In addition, the styrene moiety might be replaced with other hydrophobic groups such as diisobuthyl groups or other

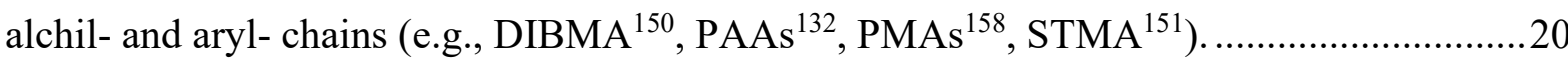

Figure 1.14. Chemical structures of SMA copolymer and its derivatives. In the past 11 years, a variety of formulation has been synthesized. Because of the maleic anhydride ring's reactivity, post-polymerization modifications were developed to expand the applicability of SMAs. Compared to SMA copolymer with equal styrene-to-maleic anhydride/acid moieties, SMA-SH ${ }^{157}$, SMA-EA ${ }^{156}$, and SMA-Glu ${ }^{164}$ formulations enable the perturbation of the charge density in the copolymer chains. SMAd-A ${ }^{155}, \mathrm{SMA}_{-} \mathrm{ED}^{155}, \mathrm{zSMA}^{154}$, and SMA-Neut ${ }^{164}$ are zwitterionic at neutral $\mathrm{pH}$ conditions. SMA-QA ${ }^{153}, \mathrm{SMI}^{152}$, and SMA-Pos ${ }^{164}$ are positively charged at neutral $\mathrm{pH}$ values. SMA-SH ${ }^{157}$ allows further functionalization with fluorescent tags, while SMA-EA-DOTA ${ }^{163}$ enables the insertion of paramagnetic ions. Finally, ST-10, and ST-100 (discussed in Chapter 5) are paramagnetic SMAs, opening to EPR, PRE- and DNPNMR studies. Table 1 summarizes a few features of each copolymer. The asterisks indicate the structures of the polymers discussed in this thesis. 
Figure 1.15. Non-SMA copolymers for membrane protein investigations. A variety of nonSMA copolymers has been proposed. DIBMA ${ }^{150}$ is widely employed in the direct extraction and stabilization of membrane proteins. AASTY ${ }^{165}$ is derived from the copolymerization of styrene and acrylic acid monomers. STMA ${ }^{151}$ uses stilbene in place of styrene monomers, tuning the rigidity of the copolymer chains. PMAs ${ }^{158}$ (formulation N-C4-60-4.7) and PAAs ${ }^{132}$ (formulations pentyl- and hexyl-PAA) were developed in the Ramamoorthy lab and have shown to be promising in membranes solubilization. Table 1.2 summarizes a few features of each copolymer. .23

Figure 1.16. SMA derivatives that form lipid nanodises. Hydrophilic functionalization to enhance polymer nanodisc features and applications. Molecular structures of SMA based polymer derivatives used to form lipid-nanodiscs (left panel) and illustrations of "sushi-like" lipid-nanodiscs (right panel). As reported in our publications ${ }^{153,155,173}$, the ability of a synthetic polymer to solubilize lipid aggregates is characterized by static light scattering (SLS) experiments on multilamellar vesicles (MLVs), TIRF based fluorescence experiments, and phosphorus-31 NMR experiments. Polymer-based nanodiscs are prepared via the selfassembly process by directly mixing an appropriate ratio of polymer and lipid(s) in a buffer. Then, the mixture is incubated and purified by size-exclusion chromatography (SEC). The purified nanodiscs are characterized by dynamic light scattering (DLS) experiments to determine the size distribution and transmission electron microscopy (TEM) images to evaluate the size homogeneity of polymer-based lipid-nanodiscs. Finally, the isotropic nanodiscs are further characterized by solution NMR spectroscopy, while the anisotropic macro-nanodiscs are analyzed using a variety of solid-state NMR experiments, including ${ }^{31} \mathrm{P},{ }^{14} \mathrm{~N}, 2 \mathrm{D}{ }^{1} \mathrm{H} /{ }^{1} \mathrm{H}$ RFDR, SLF, and 2D ${ }^{1} \mathrm{H} /{ }^{13} \mathrm{C}$ HETCOR. Based on the experience gained and different protocols used in the investigation of a variety of polymers, lipids, and proteins in the Ramamoorthy lab, the use of freeze-thaw cycles for some of the lipids, including POPC, POPG, and DSPC, is required in the preparation of polymer-based nanodiscs; further optimization may be required depending on the sample/system under investigation. In addition, sample preparation procedures for a successful reconstitution of a membrane protein need to be adapted based on the physicochemical properties of the protein under investigation. For a given membrane protein, magnetic alignment of macro-nanodiscs (large-size nanodiscs) and successful implementation of solid-state NMR experiments require optimization of various parameters, including the lipid:polymer ratio, concentration of nanodiscs, amount of paramagnetic (for example, lanthanide) salt, and temperature. Copyright 2018 Elsevier. 
Figure 1.17. pH stability of polymer nanodiscs. A schematic representation of the stability of various polymer nanodiscs under different $\mathrm{pH}$ values based on the experimental characterization as reported elsewhere ${ }^{153,155,185}$. Copyright 2018 Elsevier.

\section{Chapter 2}

Figure 2.1. The Zeeman effect and fundamentals of NMR spectroscopy. A) Shows the origin of the Zeeman effect. Giving an ensemble of spin-1/2 nuclei, in the absence of an applied magnetic field, all the spins are degenerate. If a magnetic field $\mathrm{B}_{0}$ is applied, the splitting of energy levels is generated, and the energy gap, $\Delta \mathrm{E}$, is proportional to the gyromagnetic ratio and the magnitude of the magnetic field. B) Applying a sequence of RF pulses of comparable energy to the $\Delta \mathrm{E}$, spins are excited, i.e., they flip. C) The free induction decay, FID, is then detected.

Figure 2.2. PRE and molecular systems. (A) Schematic representation of how acquisition time is affected by the recycle delay. Shorter $T_{1}$ reduces the recycle delay, shortening the experiment. (B) The dependence of relaxation times $\left(T_{1}\right.$ and $\left.T_{2}\right)$ on the tumbling rate of molecules. This scheme shows that the molecular size plays an active role in both tumbling and relaxation. An optimal range (in light green) is indicated where $T_{2}$ is not significantly affected by the decreased $T_{1}$. .50

Figure 2.3. Chemical structures of stable free-radicals and metal chelating agents commonly involved in PRE studies. (A) Nitroxide spin labels 1-oxyl-2,2,5,5-tetramethyl-3pyrroline-3-methyl-methanethiosulfonate (MTSL) and 2,2,6,6-tetramethyl-1-piperidinyloxy (TEMPO). (B) Linear metal chelators ethylenediaminetetraacetic acid (EDTA), and diethylenetriaminepentaacetic acid (DTPA). (C) Macrocyclic metal chelating agents 1,4,7triazacyclononane-1,4,7-triacetic acid (NOTA), and 1,4,7,10-tetraazacyclododecane-1,4,7,10tetraacetic acid (DOTA). .53

Figure 2.4. Schematic representation of the tagging strategies for PRE studies. (A) Direct or intramolecular labeling, (B) Indirect or intermolecular labeling, (C) Solvent PREs arising from random interactions between a macromolecule and paramagnetic co-solute molecules. Inspired by reference ${ }^{68}$. .55

Figure 2.5. $\mathrm{T}_{1}{ }^{1} \mathrm{H}$ relaxation times observed from collagen, powdered cortical bone, and demineralized bone in the absence and in the presence of Cu-EDTA $(30 \mathrm{mM})$. The $\mathrm{T}_{1}$ values were determined from ${ }^{1} \mathrm{H}$-spin-inversion recovery experiments, and the reported errors were estimated from the best-Fitting of experimental data. All measurements were performed 
on a $600 \mathrm{MHz}$ Bruker AVANCE solid-state NMR spectrometer. A, alanine; L, leucine; P, proline; E, glutamic acid; O, hydroxyproline; G, glycine; $\mathrm{CO}$, carbonyl. The signals from ( $\mathrm{P} \alpha$, $\mathrm{O} \alpha)$ and $(\mathrm{O} \alpha, \mathrm{E} \alpha)$ overlap in the ${ }^{13} \mathrm{C}$ NMR spectrum. Adapted with permission from ${ }^{100}$. Copyright 2012 American Chemical Society. .58

Figure 2.6. $2 \mathrm{D}{ }^{1} \mathrm{H} /{ }^{1} \mathrm{H}$ chemical shift correlation spectra of bicelles without (A) and with (B) $2.56 \mathrm{mM}$ copper-chelated lipid obtained under $5 \mathrm{kHz}$ MAS with total data collection times of 11 and $1.77 \mathrm{~h}$, respectively. A 6.2-fold reduction in data collection time with a similar $\mathrm{S} / \mathrm{N}$ ratio was made possible by the use of the copper-chelated lipid, as can be seen from the 1D spectral slices taken at (top) 1.34 and (bottom) $3.25 \mathrm{ppm}$ with (red) and without (black) the copperchelating lipid. An RFDR7 sequence with a $100 \mathrm{~ms}$ mixing time and a $100 \mathrm{~ms}$ low-power pulse for water saturation at $35{ }^{\circ} \mathrm{C}$ was used; $512 \mathrm{t} 1$ experiments with 32 scans were used, with recycle delays of $0.2 \mathrm{~s}$ (with copper-chelating lipid) and $2 \mathrm{~s}$ (without). (C) The structure of DMPE-DTPA (1,2-ditetradecanoyl-sn-glycero-3-phosphoethanolamine-Ndiethylenetriaminepentaacetic acid). (D) Molecular structure of DTPA (diethylenetriaminepentaacetic acid) chelated with a copper ion. DTPA is one of the common metal ion chelators. Adapted with permission from ${ }^{76}$. Copyright 2010 American Chemical Society.

Figure 2.7. (A) Representation of lipid bilayers containing a paramagnetic copper-chelated lipid and Subtilosin A, a 35-residue cyclic antimicrobial peptide that has been shown to interact with lipid bilayers with the membrane orientation depicted in (A). (B) ${ }^{15} \mathrm{~N}$ spectra of aligned 7:3 DMPC/DHPC bicelles containing 12-14\% uniformly ${ }^{15} \mathrm{~N}$ labeled (only 70-82 nmol) Subtilosin A (red) with and (black) without the $2.56 \mathrm{mM}$ copper-chelated lipid. The spectra were obtained on a $400 \mathrm{MHz}$ Varian NMR spectrometer using a ramped-amplitude crosspolarization (ramp-CP) sequence with a contact time of $0.8 \mathrm{~ms}$ under static conditions at 37 ${ }^{\circ} \mathrm{C}$. While the total data collection time was $8 \mathrm{~h}$ for both spectra, the recycle delay was different for samples without $(2 \mathrm{~s})$ and with ( $1 \mathrm{~s})$ the copper-chelated lipid. The transfer of the paramagnetic effect in $\mathrm{T}_{1}$ reduction for nuclei in the membrane via proton spin diffusion is also indicated in (A). (C) Primary structure of the antimicrobial peptide Subtilosin A. Adapted with permission from ${ }^{76}$. Copyright 2010 American Chemical Society.

Figure 2.8. Four-fold increase in the sensitivity of 2D SOFAST-HMQC experiments. (A) 2D SOFAST- ${ }^{1} \mathrm{H} /{ }^{15} \mathrm{~N}-\mathrm{HMQC}$ spectrum of a $9.3 \mathrm{mM}$ (unlabeled) MSI-78 (also known as pexiganan) incorporated in DMPC/DHPC isotropic bicelles $(\mathrm{q}=[\mathrm{DMPC}] /[\mathrm{DHPC}]=0.25$, DMPC: 1,2- dimyristoyl-sn-glycero-3-phosphocholine, DHPC: 1,2-dihexanoyl-sn-glycero-3phosphocholine) containing a $2.96 \mathrm{mM}$ copper-chelated DMPE lipid. (B) 3D structure of MSI- 
78 embedded in bicelles along with its amino acid sequence. (C) Signal-to-noise ratio obtained from 2D SOFAST-HMQC spectra of a $9.3 \mathrm{mM}$ unlabeled MSI-78 in $\mathrm{q}=0.5$ isotropic bicelles without copper-chelated lipid (black) and with a $2.96 \mathrm{mM}$ copper-chelated lipid (red). (D) 1D ${ }^{1} \mathrm{H}$ chemical shift projection spectrum obtained from $2 \mathrm{D}$ SOFAST- ${ }^{1} \mathrm{H} /{ }^{15} \mathrm{~N}-\mathrm{HMQC}$ spectra that were obtained with no copper (black) and a $2.96 \mathrm{mM} \mathrm{Cu}^{2+}$-DMPE-DTPA (red). All spectra presented in this study were obtained from a $900 \mathrm{MHz}$ Bruker NMR spectrometer at $35{ }^{\circ} \mathrm{C}$ using a cryoprobe. Each 2D SOFAST-HMQC spectrum was obtained from 64 t1 experiments, 256 scans, and a $100 \mathrm{~ms}$ recycle delay; the total data collection time (including the acquisition time and delays in INEPT) was $\sim 54 \mathrm{~min}$. Adapted with permission from ${ }^{45}$. Copyright 2011 American Chemical Society.

Figure 2.9. Site-specific ${ }^{1} H T_{1}$ relaxation times of the backbone resonances of $\left[\mathrm{U}-{ }^{15} \mathrm{~N}\right]-$ SLN oriented in lipid bicelles with $5 \% \mathrm{Cu}^{2+}$-chelated lipids measured by the 2D inversion recovery SAMPI4 experiment. B) Mapping of the site-specific $T_{1}$ relaxation times on SLN structure (blue), where unobservable/overlapped residues in the SAMPI4 spectrum are shown in white. C) 2D SE-SAMPI4 spectra of [U- $\left.{ }^{15} \mathrm{~N}\right]-\mathrm{SLN}$ without paramagnetic and d) with $5 \%$ $\mathrm{Cu}^{2+}$-chelated lipids. The average ${ }^{15} \mathrm{~N}$ linewidths for isolated peaks are $82.9 \mathrm{~Hz}$ and $86.7 \mathrm{~Hz}$ without and with $5 \% \mathrm{Cu}^{2+}$-chelated lipids, respectively. Adapted with permission from ${ }^{151}$. Copyright 2018 Elsevier. 62

\section{Chapter 3}

Figure 3.1. Reaction scheme for the synthesis of SMA-EA-DOTA: 1) The nucleophilic ring opening reaction using DOTA-t-butyl-acetate. 2) Nucleophilic ring opening reaction using ethanolamine. 3) TFA deprotection of DOTA

Figure 3.2. FT-IR spectra of copolymers. a) SMAnh (gray), SMA-EA (red), and SMA-EADOTA (blue); b) Expanded regions showing the $\mathrm{C}=\mathrm{O}$ stretching frequency $\left(2000-1400 \mathrm{~cm}^{-1}\right)$.

Figure 3.3. DLS of nanodiscs. DLS profiles of $1: 1 \mathrm{w} / \mathrm{w}$ DMPC:SMA-EA (in red) and DMPC:SMA-EA-DOTA (in blue). a) The mass profiles, and b) intensity profiles. Both were obtained on samples passed through SEC.

Figure 3.4. Static Light Scattering metal titrations. SLS traces obtained by titrating $1 \mathrm{mg}$ of SMA-EA nanodiscs (left column) and SMA-EA-DOTA nanodiscs (right column) with a solution of varying metal ion concentration. The concentration values were obtained by fitting the experimental points, obtained in triplicate. 
Figure 3.5. Characterization of polymers and polymer nanodiscs. a) Molecular structures of SMA-EA and SMA-EA-DOTA. b) FT-IR spectra of the starting material (SMAnh) and synthetic polymers. FT-IR results confirm the functionalization of the starting material (SMAnh in dark gray) and similarities among SMA-EA (in red) and SMA-EA-DOTA (in blue). Full spectra are included in Figure 3.2. c) Dissolution of multilamellar vesicles (MLVs) by SMA-EA (red) and by SMA-EA-DOTA (blue) for a 1:1 lipid:polymer weight ratio. d) Stability of DMPC:SMA-EA 1:1 w/w and DMPC: SMA-EA-DOTA 1:1 w/w macro-nanodiscs against different metal ions. Size exclusion chromatography (e) and DLS (f) profiles for 1:1 w/w ratio of DMPC:SMA-EA and DMPC:SMA-EA-DOTA samples. The DLS profiles were obtained after SEC purification for the nanodiscs fraction highlighted in (e). The intensity profile can be found in Figure 3.4.

Figure 3.6. Use of paramagnetic metal-chelated polymers to speed-up NMR data acquisition. A schematic representation of how SMA-EA-DOTA copolymer-based nanodiscs chelated with paramagnetic metals can be used to reduce $\mathrm{T}_{1}$ relaxation times and to shorten the recycle delay between the successive scans in NMR data acquisition. .96

Figure 3.7. NMR spectra of nanodiscs in the presence of $\mathbf{G d}^{3+}$ ions. a) Schematic representation of a macro-nanodisc. b) Molecular structure of 1,2-dimyristoyl-sn-glycero-3phosphocholine (DMPC). c) ${ }^{1} \mathrm{H}$ NMR spectra of 1:1 w/w DMPC:SMA-EA (red) and DMPC:SMA-EA-DOTA (blue) macro-nanodiscs. d) ${ }^{1} \mathrm{H}$ NMR spectra of 1:1 w/w DMPC: SMA-EA (red) and SMA-EA-DOTA (blue) nanodiscs titrated with the indicated amount of $\mathrm{Gd}^{3+}$ ions. All NMR were obtained using $4 \mathrm{mg}$ of lipids in $10 \mathrm{mM}$ phosphate buffer $(\mathrm{pH}=7.4)$ in $100 \% \mathrm{D}_{2} \mathrm{O}$ at $35^{\circ} \mathrm{C}$.

Figure 3.8. Measurement of $\mathbf{T}_{1}$ for protons. Spin-inversion NMR experimental data obtained from 1:1 w/w DMPC:SMA-EA-DOTA nanodiscs to determine $\mathrm{T}_{1}$ values of protons for varying concentrations of $\mathrm{Gd}^{3+}$ as indicated. Equation 1 was used to obtain the best-fitting values given in Table 1. .98

Figure 3.9. Efficiency of paramagnetic metal ions in shortening $T_{1} \cdot T_{1}$ comparison among the different investigated metal ions at $\left[\mathrm{Ln}^{3+}\right]=0.5 \mathrm{mM}$. The black bar represents the data obtained from a control sample, 1:1 (w/w) DMPC: SMA-EA-DOTA without paramagnetic metal ions. Data shown for ${ }^{1} \mathrm{H}$ peaks of a) styrene/aromatic group, b) $\gamma$ methyl groups in the quaternary ammonium of DMPC, c) the methylene groups from the acyl chains (C4-C13) of DMPC, and d) the terminal acyl-chain methyl groups (C14) of DMPC. Each of the NMR samples used in these measurements consisted of $4 \mathrm{mg}$ of DMPC in $10 \mathrm{mM}$ Phosphate buffer $\mathrm{pH}=7.4$ in $\mathrm{D}_{2} \mathrm{O}$. 100 


\section{Chapter 4}

Figure 4.1. Pyrene essay on copolymer solutions. Panels on the left show a selection of pyrene fluorescent spectra in the presence of various concentrations of copolymers. Gray arrows highlight the variation in the fluorescence intensity of the peaks $I_{1}$ and $I_{3}$ for varying the concentration of copolymer. The change is consistent among the whole spectrum. Panels on the right show the $I_{1} / I_{3}$ average of three independent experiments against the decimal logarithm of the polymer concentration (in $\mathrm{mg} / \mathrm{mL}$ ). Error bars correspond to the standard deviation among them. The yellow arrow points at the flex $\left(\mathrm{x}_{0}\right)$ of each curve obtained using a sigmoidal fitting 115

Figure 4.2. Pyrene essay on unpurified copolymer-lipid nanodiscs solutions as a function of the copolymer concentration $(\mathbf{m g} / \mathbf{m L})$. Panels on the left show a selection of pyrene fluorescent spectra in the presence of various concentrations of copolymers. Gray arrows highlight the variation in the fluorescence intensity of the peaks $I_{1}$ and $I_{3}$ for varying the concentration of copolymer. The change is consistent among the whole spectrum. Panels on the right show the $\mathrm{I}_{1} / \mathrm{I}_{3}$ average of three independent experiments against the decimal logarithm of the polymer concentration (in $\mathrm{mg} / \mathrm{mL}$ ). Error bars correspond to the standard deviation among them. The yellow arrow points at the flex $\left(\mathrm{x}_{0}\right)$ of each curve obtained using a sigmoidal fitting. 116

Figure 4.3. Pyrene essay on unpurified copolymer-lipid nanodiscs solutions as a function of phospholipids concentration (mM). Panels on the left show a selection of pyrene fluorescent spectra in the presence of various concentrations of copolymers. Gray arrows highlight the variation in the fluorescence intensity of the peaks $I_{1}$ and $I_{3}$ for varying the concentration of copolymer. The change is consistent among the whole spectrum. Panels on the right show the $I_{1} / I_{3}$ average of three independent experiments against the decimal logarithm of the lipid concentration (in $\mathrm{mM}$ ). Error bars correspond to the standard deviation among them. The yellow arrow points at the flex $\left(\mathrm{x}_{0}\right)$ of each curve obtained using a sigmoidal fitting.

Figure 4.4. Pyrene essay on SEC-purified copolymer-lipid nanodiscs solutions as a function of phospholipids concentration (mM). Panels on the left show a selection of pyrene fluorescent spectra in the presence of various concentrations of copolymers. Gray arrows highlight the variation in the fluorescence intensity of the peaks $I_{1}$ and $I_{3}$ for varying the concentration of copolymer. The change is consistent among the whole spectrum. Panels on the right show the $\mathrm{I}_{1} / \mathrm{I}_{3}$ average of three independent experiments against the decimal logarithm 
of the lipid concentration (in $\mathrm{mM}$ ). Error bars correspond to the standard deviation among them. The yellow arrow points at the flex $\left(\mathrm{x}_{0}\right)$ of each curve obtained using a sigmoidal fitting.

Figure 4.5. Size Exclusion Chromatograms of 1:1 w/w copolymer-DMPC nanodiscs. For each sample, the first peak is associated with the copolymer-lipid nanodiscs, while the second peak is associated with the micellar species formed by each copolymer in solution. 119

Figure 4.6. ${ }^{1}$ H-NMR spectra of SMA-EA:DMPC 1:1 (w/w) nanodiscs. Samples are recorded in the presence of $10 \mathrm{mM}$ sodium cholate. To assess the molar concentration, the peak associated with quaternary ammonium protons from DMPC $(\sim 3.3 \mathrm{ppm})$ is integrated vs. the peak at $\sim 0.75 \mathrm{ppm}$ from the cholate.

Figure 4.7. ${ }^{1} \mathrm{H}-\mathrm{NMR}$ spectra of SL25010 P:DMPC 1:1 (w/w) nanodiscs. Samples are recorded in the presence of $10 \mathrm{mM}$ sodium cholate. To assess the molar concentration, the peak associated with quaternary ammonium protons from DMPC ( $\sim 3.3 \mathrm{ppm})$ is integrated vs. the peak at $\sim 0.75 \mathrm{ppm}$ from the cholate.

Figure 4.8. ${ }^{1} \mathrm{H}-\mathrm{NMR}$ spectra of SL30010 P:DMPC 1:1 (w/w) nanodiscs. Samples are recorded in the presence of $10 \mathrm{mM}$ sodium cholate. To assess the molar concentration, the peak associated with quaternary ammonium protons from DMPC ( $\sim 3.3 \mathrm{ppm})$ is integrated vs. the peak at $\sim 0.75 \mathrm{ppm}$ from the cholate.

Figure 4.9. ${ }^{1} \mathrm{H}-\mathrm{NMR}$ spectra of SL40005 P:DMPC 1:1 (w/w) nanodiscs. Samples are recorded in the presence of $10 \mathrm{mM}$ sodium cholate. To assess the molar concentration, the peak associated with quaternary ammonium protons from DMPC $(\sim 3.3 \mathrm{ppm})$ is integrated vs. the peak at $\sim 0.75$ ppm from the cholate.

Figure 4.10. ${ }^{1} \mathrm{H}-\mathrm{NMR}$ spectra of $10 \mathrm{mM}$ Sodium Cholate in $100 \% \mathrm{D} 2 \mathrm{O}$. The peak used for integration is at $0.75 \mathrm{ppm}$, corresponding to the methyl group $\mathrm{C} 18$. 122

Figure 4.11. Raman vibrational spectrum of the $1 \mathrm{mg} / \mathrm{mL}$ unpurified SMA-EA:DMPC 1:1 (w/w) nanodiscs solution sample shown in Figure 4.15. 123

Figure 4.12. Chemical structures of SMA-based copolymers and copolymer-lipid nanodiscs. a) Chemical structured of the copolymers used in this study. The chemical structure of XIRAN ${ }^{\circledR}$ copolymers (SL25010 P, SL30010 P, SL40005 P) by Polyscience ${ }^{\circledR}$ are shown on the left and differ from each other for molecular weight and styrene-to-maleic acid (x:y) molar ratio. SMA-EA is a functionalized, low-molecular-weight derivative of SMA with a reduced charge density ${ }^{36}$. More details are provided in Table 1. All structures are shown in their fully protonated form. b) Simplified schematic representation of the chemical equilibrium between «nanodisc-bound» and «unbound/free-micellar» copolymer chains. 
Figure 4.13. Pyrene fluorescence spectra for (a) «free» copolymers and (b) copolymerlipid nanodiscs upon excitation at $331.5 \mathrm{~nm}$. a) Fluorescence spectra of pyrene in the presence of SMA-EA (black line), SL25010 P (red line), SL30010 P (blue line), or SL40005 (green line) copolymers «free» in solution and at the highest concentration used in this study. b) Fluorescence spectra of pyrene in the presence of unpurified SMA-EA-based nanodiscs (black line), SL25010 P based nanodiscs (red line), SL30010 P based nanodiscs (blue line), or SL40005 P (green line) at the highest concentration used in this study. In purple, both a) and b) shows the fluorescence spectrum of $1 \mu \mathrm{M}$ pyrene and can be associated with the most diluted sample, or the «infinite dilution sample.» Each sample was prepared in $10 \mathrm{mM}$ ammonium acetate buffer at $\mathrm{pH} 7$ at $25^{\circ} \mathrm{C}$, recorded at $25^{\circ} \mathrm{C}$, and pyrene concentration was kept constant at $1 \mu \mathrm{M}$.

Figure 4.14. Copolymers show drastic changes in the presence of phospholipids. a) Plots of the pyrene $\mathrm{I}_{1} / \mathrm{I}_{3}$ ratio against the decimal logarithm of the copolymers' concentration in $\mathrm{mg} / \mathrm{mL}$. As expected from the physicochemical properties listed in Table 4.1, the polymer SL25010 P (circles in red) results in the most hydrophobic, while SL40005 P (triangles in green) is the most hydrophilic. SMA-EA (black squares) and SL30010 P (blue triangles) show comparable responses. b) Plots of the pyrene $\mathrm{I}_{1} / \mathrm{I}_{3}$ ratio against the concentration of copolymerlipid nanodiscs in solution. The concentration is reported as a function of the polymer concentration's decimal logarithm in $\mathrm{mg} / \mathrm{mL}$. Interestingly, the differences observed in a) become negligible among all the copolymers when interacting with lipids. Both a) and b) show the average of three experiments obtained from independent samples. Each sample was prepared in $10 \mathrm{mM}$ ammonium acetate buffer at $\mathrm{pH} 7$ at $25^{\circ} \mathrm{C}$, recorded at $25^{\circ} \mathrm{C}$, and pyrene concentration was kept constant at $1 \mu \mathrm{M}$. Data, error bars, and fitting are reported in Figures 4.1 and 4.2 .

Figure 4.15. AFM images and depth profiles above and below the c.m.c. of SMA-EA copolymer. a) AFM image of a sample of $1 \mathrm{mg} / \mathrm{mL}$ DMPC:SMA-EA 1:1 w/w. I) and II) are the depth profiles indicated in a). b) AFM image of a sample of $0.001 \mathrm{mg} / \mathrm{mL}$ DMPC:SMAEA 1:1 w/w. III) and IV) are the depth profiles indicated in b). The Raman spectrum is shown in Figure 4.11. 128

Figure 4.16 - Comparison of the pyrene $I_{1} / I_{3}$ ratio against the concentration of unpurified (squares) and purified (circles) copolymer-nanodiscs in solution. By purification through SEC, removing the micellar polymer not bound to the nanodiscs edge causes a consistent horizontal shift for all of the copolymer formulations investigated, as reported from a) to d). The micellar polymer removed by SEC is either an excess or in chemical equilibrium among 
the states «nanodiscs-bound» and «unbound/free-micellar» in solution. Each sample was prepared in $10 \mathrm{mM}$ ammonium acetate buffer at $\mathrm{pH} 7$ at $25^{\circ} \mathrm{C}$, recorded at $25^{\circ} \mathrm{C}$, and pyrene concentration was kept constant at $1 \mu \mathrm{M}$. Data, error bars, and fitting are reported in Figures 4.3 and 4.4. The concentration of SEC-purified copolymer-lipid nanodiscs was assessed through ${ }^{1} \mathrm{H}$ NMR using sodium cholate as an internal standard and reported in Figures 4.6-4.9. 130

\section{Chapter 5}

Figure 5.1. Synthesis of ST-10 and ST-100 copolymers. The figure shows the scheme of reaction to obtain both ST-10 and ST-100 copolymers from SMAnh starting material. The synthesis involves a nucleophilic ring-opening reaction. For ST-10, step 1 involves the addition of $\sim 10-15 \%$ of 4 -amino-TEMPO before treatment with an excess of ethanolamine (EA). ST100 involves a 1-step functionalization with an excess of 4-amino-TEMPO. Both copolymers are synthesized in anhydrous NMP, using triethylamine $\left(\mathrm{Et}_{3} \mathrm{~N}\right)$ as the base.

Figure 5.2. Full FT-IR spectra of SMAnh, SMA-EA, ST-10, and ST-100 copolymers. 142 Figure 5.3. Characterization of polymers and polymer nanodiscs. a) Shows the structures of the SMAnh (the starting material), SMA-EA, ST-10, and ST-100 copolymers. b) FT-IR spectra of the starting material (SMAnh - purple), SMA-EA (light blue), ST-10 (orange), and ST-100 (red). FT-IR results confirm the functionalization of the SMAnh and show similarities among SMA-EA, ST-10, and ST-100. Full spectra are presented in Figure 5.2. 145

Figure 5.4. CW-EPR spectra of copolymers and copolymer nanodiscs. The top panels show data from a solution of ST-10 (top-left panel) and nanodiscs with DMPC in a 2:1 w/w ratio (top-right panel). The bottom panels show a solution of ST-100 (bottom-left panel) and ST100:DMPC nanodiscs in a 2:1 w/w ratio (bottom-right panel) 146

Figure 5.5. ${ }^{1} \mathrm{H}$ NMR spectra of paramagnetically labeled nanodiscs varying the free radical concentration. a) shows ${ }^{1} \mathrm{H}$ spectra of 1,2 , 4, and $10 \mathrm{mg}$ ST-10:DMPC $(1: 1 \mathrm{w} / \mathrm{w})$ nanodiscs. Significant broadening effects are observed for the $10 \mathrm{mg}$ sample. b) shows the ${ }^{1} \mathrm{H}$ spectra of 1, 2, 4, and $10 \mathrm{mg}$ of ST-100:DMPC (1:1 w/w) nanodiscs. Due to the larger concentration of free radicals in ST-100, broadening effects are more prominent, starting from lower copolymer concentrations.

Figure 5.6. Measurement of $\mathbf{T}_{1}$ for protons. The figure shows the experimental data obtained inversion recovery NMR experiments for 1:1 w/w DMPC:ST-10 nanodiscs (top) and 1:1 w/w DMPC:ST-100 nanodiscs (bottom) at $35^{\circ} \mathrm{C}$ for both to determine $\mathrm{T}_{1}$ values of protons varying 
the free radicals concentrations as indicated. Equation 1 from Chapter 3 was used to obtain the best-fitting values.

Figure 5.7. A comparison of $T_{1}$ for protons of ST-10 and ST-100 based nanodiscs. Spininversion NMR experimental data obtained from $4 \mathrm{mg}$ 1:1 w/w ST-10:DMPC and ST100:DMPC nanodiscs to determine $T_{1}$ values of protons. Equation 1 from chapter 3 was used to obtain the best-fitting values given in Tables 1-3, chapter 5 .

Figure 5.8. Efficiency in paramagnetic labeling of copolymer nanodiscs. Graphical comparison of the $T_{1}$ values reduction in the $1: 1 \mathrm{w} / \mathrm{w}$ copolymer-lipid nanodiscs reported in this thesis. SMA-EA-DOTA loaded with $\left[\mathrm{Gd}^{3+}\right]=0.5 \mathrm{mM}$ (reported in chapter 3), $4 \mathrm{mg}$ of ST10 , and $4 \mathrm{mg}$ of ST-100 all show $\mathrm{T}_{1}$ reduction because of the presence of paramagnetic labels in the outer rim of the copolymer-nanodiscs. Values are expressed in percentages for the diamagnetic SMA-EA copolymer $\left(\mathrm{T}_{1} 100 \%\right)$. 152

\section{Chapter 6}

Figure 6.1. Examples of nanomedicine. Different applications of nanoparticles involved in therapy and diagnosis. Figure reproduced with permission from reference ${ }^{5}$. Copyright 2017 Dove Press.

Figure 6.2. Structure of liposomes for drug delivery and diagnostics. Liposomes are colloidal drug carriers consisting of a phospholipid bilayer surface enclosing an aqueous core. Hydrophilic components can be entrapped inside the aqueous core, while the lipophilic components can be incorporated between the lipid bilayers. On the liposomes surface, different particles that target the interest cells can be attached. To avoid the immune system response, the liposomes surface is loaded with a polymer called polyethylene glycol. Thus, the cargo is protected and is discharged into the target cells. Reproduced with permission from reference ${ }^{5}$. Copyright 2017 Dove Press.

Figure 6.3. Polymer-based nanomedicine. Schematic showing: panel (a) the relationship between nanomedicines and polymer therapeutics, and typical structures of polymer conjugates and their routes of administration. Reproduced with permission from reference ${ }^{3}$. Copyright 2011 Elsevier. 161

Figure 6.4. Schematics of the EPR effect. Passive targeting relies on cell-specific functions or local environments specific to target the tissue to facilitate uptake and accumulation in tumor tissues and inflammatory sites. Reproduced with permission from reference ${ }^{5}$. Copyright 2017 Dove Press. 
Figure 6.5. SMA-EA-DOTA:lipids nanodiscs for magnetic resonance imaging. The HDLlike supramolecular architecture and the self-assembling nature makes copolymer nanodiscs good MRI CA candidates. All the components are considered to show low toxicity. 165 Figure 6.6 - Gd $^{3+}$-loaded DMPC:SMA-EA-DOTA 1:1 w/w nanodises as macromolecular CAs. The figure shows a comparison of the $T_{1}$ and $T_{2}$ times reduction of samples of DMPC:SMA-EA 1:1 (w/w) (top panels) and DMPC:SMA-EA-DOTA 1:1 (w/w) (bottom panels) in the presence of $\mathrm{Gd}^{3+}$-ions. As seen in the figure, chelated $\mathrm{Gd}^{3+}$ ions (bottom panels) affect $T_{1}$ and $T_{2}$ differently than the free in solution counterpart (top panel) 166 Figure 6.7 - $\mathrm{Mn}^{2+}$-loaded DMPC:SMA-EA-DOTA 1:1 w/w nanodiscs as macromolecular CAs. The figure shows a comparison of the $T_{1}$ and $T_{2}$ times reduction of samples of DMPC:SMA-EA-DOTA 1:1 (w/w) loaded with different concentrations of $\mathrm{Mn}^{2+}$ ions. 167

\section{Appendix}

Figure S1. T1 measurements for DMPC: SMA-EA 1:1 (w/w) macro-nanodiscs. a) the stacking of $1 \mathrm{D}{ }^{1} \mathrm{H}-\mathrm{NMR}$ spectra in the presence of different concentrations of $\left[\mathrm{Gd}^{3+}\right]$ ions. $\mathrm{b}$ ), c), d), and e) inversion recovery fitting plots of data for the selected ${ }^{1} \mathrm{H}$ peaks at various $\left[\mathrm{Gd}^{3+}\right]$ concentrations.

Figure S2. T1 measurements for DMPC: SMA-EA-DOTA 1:1 (w/w) macro-nanodiscs. a) the stacking of $1 \mathrm{D}{ }^{1} \mathrm{H}-\mathrm{NMR}$ spectra in the presence of different concentrations of $\left[\mathrm{Dy}^{3+}\right]$ ions. b), c), d), and e) inversion recovery plots for the selected ${ }^{1} \mathrm{H}$ peaks at various $\left[\mathrm{Dy}^{3+}\right]$ concentrations

Figure S3. T1 measurements for DMPC: SMA-EA-DOTA 1:1 (w/w) macro-nanodiscs. a) the stacking of $1 \mathrm{D}{ }^{1} \mathrm{H}-\mathrm{NMR}$ spectra in the presence of different concentrations of $\left[\mathrm{Er}^{3+}\right]$ ions. b), c), d), and e) inversion recovery plots for the selected ${ }^{1} \mathrm{H}$ peaks at various $\left[\mathrm{Er}^{3+}\right]$ concentrations.

Figure S4. T1 measurements for DMPC: SMA-EA-DOTA 1:1 (w/w) macro-nanodiscs. a) the stacking of $1 \mathrm{D}{ }^{1} \mathrm{H}-\mathrm{NMR}$ spectra in the presence of different concentrations of $\left[\mathrm{Eu}^{3+}\right]$ ions. b), c), d), and e) inversion recovery fitting plots for the selected ${ }^{1} \mathrm{H}$ peaks at various $\left[\mathrm{Eu}^{3+}\right]$ concentrations.

Figure S5. T1 measurements for DMPC: SMA-EA-DOTA 1:1 (w/w) macro-nanodiscs. a) the stacking of $1 \mathrm{D}{ }^{1} \mathrm{H}-\mathrm{NMR}$ spectra in the presence of different concentrations of $\left[\mathrm{Yb}^{3+}\right]$ ions. b), c), d), and e) inversion recovery plots for the selected ${ }^{1} \mathrm{H}$ peaks at various $\left[\mathrm{Yb}^{3+}\right]$ concentrations. 
Figure S6. NMR spectra of SMA-EA-DOTA in the presence of paramagnetic metals. Stacking of 1D ${ }^{1} \mathrm{H}-\mathrm{NMR}$ of DMPC:SMA-EA-DOTA 1:1 (w/w) macro-nanodiscs in the presence of different $\mathrm{Ln}^{3+}$ ions. Lanthanide trivalent ions such as $\mathrm{Eu}^{3+}, \mathrm{Gd}^{3+}, \mathrm{Dy}^{3+}, \mathrm{Er}^{3+}, \mathrm{Yb}^{3+}$ are respectively shown in a), b), c), d), and e). 185

Figure S7. Normalized fitting (left panel) and normalized $T_{1}$ values (right panel) for DMPC: SMA-EA-DOTA 1:1 (w/w) macro-nanodises in the presence of $0.5 \mathrm{M} \mathrm{Ln}^{3+}$. From top to bottom data relative to the aromatic, gamma, 2-13, and $14{ }^{1} \mathrm{H}$ peaks 186 


\section{LIST OF TABLES}

\section{Chapter 1}

Table 1.1. List of SMA-based copolymers. A list of SMA-based copolymers is shown in chronological order, from the first appearance of SMA to extract membrane proteins to the latest. The table does not show RAFT variants of CSTR synthesized copolymers and does not include formulations showing different styrene-to-maleic acid ratios. ST-10 and ST-100 will be discussed in Chapter 5

Table 1.2. List of non-SMA-based copolymers. A list of non-SMA-based copolymers is shown in chronological order of appearance. The table does not show RAFT variants of CSTR synthesized copolymers and does not include formulations showing different subunit (hydrophobic-to-hydrophilic) molar ratios .23

\section{Chapter 3}

Table 3.1. T 1 values of DMPC: SMA-EA-DOTA 1:1 (w/w) macro-nanodiscs for varying $\left[\mathbf{G d}^{3+}\right]$. Values marked as N/D are data not determined due to significant line-broadening because of the presence of paramagnetic ions.

\section{Chapter 4}

Table 4.1. Physicochemical data of the investigated SMA copolymers. Spanning a range of molecular weights that goes from $\sim 2$ to $\sim 10 \mathrm{kDa}$, each copolymer has comparable dispersity $\left(Đ=M_{w} / M_{n}>2.5\right)$ but different hydrophobicity/hydrophilicity ratios, as suggested from the styrene-to-maleic acid ratio. Additionally, SMA-EA shows a modified charge-density because of its modification if compared to any SMA-equivalent. The experimental curves $I_{1} / I_{3}$ vs. Log C shown in Figure 4.13a were fitted to a sigmoidal function. The c.m.c. values for each 
copolymer are obtained by taking the flex $\left(\mathrm{x}_{0}\right)$ of each fitted curve and converted from a decimal logarithmic scale to a linear scale. The coefficient of determination, $\mathrm{R}^{2}$, is also shown for each fitting. Experimental conditions are available in the «Materials and Methods» section

Table 4.2. Copolymer-lipid nanodiscs data: unpurified vs. purified. From left-to-right, the chart shows the data plotted, respectively, in Figures 4.2-4.4. The polymer-based nanodiscs were obtained by mixing the lipids and the copolymers in a 1:1 weight ratio, as detailed in the «Materials and Methods» section. Unpurified nanodiscs appear twice; as a function of the polymer concentration $(\mathrm{C}$ in $\mathrm{mg} / \mathrm{mL})$ and as a function of the DMPC concentration $(\mathrm{mM})$. The «c.m.c.-like» values for each copolymer-nanodiscs were obtained by taking the flex $\left(\mathrm{x}_{0}\right)$ of each fitted curve and converted from a decimal logarithmic scale to a linear range. The coefficient of determination, $\mathrm{R}^{2}$, is also shown for each fitting.

\section{Chapter 5}

Table 5.1. T1 values for SMA-EA:DMPC $(1: 1 \mathrm{w} / \mathrm{w})$ nanodiscs varying the free radicals' concentration in the sample. Highlighted in gray is the condition used in the experiments shown in chapter 3 148

Table 5.2. $T_{1}$ values for ST-10:DMPC $(1: 1 \mathrm{w} / \mathrm{w})$ nanodises varying the free radicals' concentration in the sample. Highlighted in gray is the condition used in the experiments shown in chapter 3 . The values labeled with the asterisks come from significantly broad peaks and not entirely reliable. Figure 5.3 shows the associated ${ }^{1} \mathrm{H}-\mathrm{NMR}$ spectrum 150

Table 5.3. $T_{1}$ values for $S T-100: D M P C(1: 1 \mathrm{w} / \mathrm{w})$ nanodiscs varying the free radicals' concentration in the sample. Highlighted in gray is the condition used in the experiments shown in chapter 3 . The values labeled with the asterisks come from significantly broad peaks and not entirely reliable. Figure 5.3 shows the associated ${ }^{1} \mathrm{H}-\mathrm{NMR}$ spectrum 


\section{Chapter 6}

Table 6.1. Chemical characteristics of Gadolinium-based contrast agents. Data were taken from reference ${ }^{64}$

\section{Appendix}

Table S1. T1 values measured from DMPC:SMA-EA 1:1 (w/w) macro-nanodiscs for varying $\left[\mathbf{G d}^{3+}\right]$. Values marked as N/D are data not determined due to significant linebroadening due to the presence of paramagnetic ions

Table S2. T1 values of DMPC: SMA-EA-DOTA 1:1 (w/w) macro-nanodiscs for varying $\left[\mathbf{D y}^{3+}\right]$. Values marked as N/D are data not determined due to significant line-broadening due to the presence of paramagnetic ions 181

Table S3. T1 values of DMPC: SMA-EA-DOTA 1:1 (w/w) macro-nanodises for varying $\left[\mathbf{E r}^{3+}\right]$. Values marked as N/D are data not determined due to significant line-broadening due to the presence of paramagnetic ions 182

Table S4. T1 values of DMPC:SMA-EA-DOTA 1:1 (w/w) macro-nanodises for varying $\left[\mathbf{E u}^{3+}\right]$. Values marked as N/D are data not determined due to significant line-broadening due to the presence of paramagnetic ions

Table S5. T1 values of DMPC:SMA-EA-DOTA 1:1 (w/w) macro-nanodises for varying $\left[\mathbf{Y b}^{3+}\right]$. Values marked as N/D are data not determined due to significant line-broadening due to the presence of paramagnetic ions 184 


\section{ABSTRACT}

Membrane proteins are critical components of any cell, and their malfunction is associated with numerous diseases. For this reason, they represent a primary target for various drugs on the market, but both academic and pharmaceutical research is hindered by the challenges associated with obtaining stable and functioning samples.

Artificial lipid membranes are crucial for the investigation of membrane proteins because of their ability to simulate the amphipathic native-like environment of the cell membrane. Recent studies have shown the dramatic advantages of using lipid/nanodiscs as compared to other types of membrane mimetics. While the nanodiscs prepared using scaffold proteins, peptides, and proteins have their advantages and limitations; there is significant interest in synthetic polymers because of the broad scope and feasibilities. Macromolecules such as copolymers of styrene and maleic acid (SMA) interact with lipids forming stable discoidal nanoparticles made of bilayer patches wrapped by the polymeric belt. These copolymers have also been used to extract membrane proteins directly from their native environment and isolate them into nanodiscs without using detergents. Despite the many successes reported in the literature, copolymer-nanodiscs still show several limitations, and new formulations are under development. The Ramamoorthy research group focused on the hydrophilic functionalization of a low molecular-weight SMA copolymer. This approach allowed for the tuning and enhancement of these polymers, particularly in the field of nuclear magnetic resonance (NMR) spectroscopy.

NMR is widely employed to study nanodiscs reconstituted membrane proteins but suffers from its intrinsic low sensitivity, which necessitates long data acquisition times. Paramagnetic resonance enhancement (PRE) is among the strategies that have been used to enhance the sensitivity of NMR by reducing the spin-lattice relaxation or $\mathrm{T}_{1}$, a key parameter in assessing the duration of the required data acquisition. However, PRE requires the introduction of PREagents in the sample that could alter the sample's stability and function. 
This thesis reports a novel PRE-agent that does not involve (i) direct labeling of membrane proteins, (ii) the alteration of the surrounding lipid composition, or (iii) the presence of free metal ions in the sample. Specifically, SMA-EA-DOTA copolymer allows the chelation of paramagnetic ions directly in the copolymer-lipid nanodiscs' outer rim without contaminating the nanodiscs' constituents such as lipids and proteins, enabling $\mathrm{T}_{1}$-reduction. A variety of lanthanide ions are investigated to quantify the PRE effects and for use in nanodiscs-enabled studies on membrane systems.

Since nanodiscs-forming copolymers act, de facto, as macromolecular detergents, this thesis also investigates the relationship between the critical micelle concentration (c.m.c.) of a set of SMA-copolymers and their ability to form nanodiscs. It was found that the interaction with phospholipids alters the copolymers' c.m.c. values, and the existence of an equilibrium between the «free» or «micellar» copolymer chains and the «nanodiscs-bound» copolymer chains. Because of this equilibrium, the thesis speculates the possibility of substituting inexpensive copolymers after membrane proteins isolation and purification with paramagnetically-tagged copolymers for magnetic resonance studies. Aside from PRE-NMR, membrane proteins reconstituted in paramagnetically-labeled nanodiscs, such as SMA-EA-DOTA, ST-10, ST-100, and mixed formulations, can be studied using other biophysical techniques including electron paramagnetic resonance and dynamic nuclear polarization NMR.

Finally, paramagnetically-tagged copolymer nanodiscs can find new applications outside the biophysical and biochemical fields. For instance, these bioinspired paramagnetic nanoparticles might find applications in the fields of drug delivery and magnetic resonance imaging as macromolecular contrast agents for better diagnosis of solid tumors. 


\section{Chapter 1}

\section{Introducing SMALP for the Study of Membrane Proteins}

The content for this chapter is partially included in the review article:

Ravula Thirupathi, Hardin Nathaniel Z., Di Mauro Giacomo M., Ramamoorthy Ayyalusamy, Styrene maleic acid derivatives to enhance the applications of bio-inspired polymer based lipidnanodiscs, European Polymer Journal, 108, 11/2018, 597-602,

DOI: $10.1016 /$ j.eurpolymj.2018.09.048 


\subsection{Outline of this thesis}

The study of structure, dynamics, and function of membrane proteins through biophysical and biochemical approaches heavily relies on membrane mimetics. Among the various alternatives, lipid nanodiscs are the latest development in the field and are increasingly used for multiple applications. Lipid-nanodiscs are lipid bilayer patches surrounded by an amphipathic belt and can be formed using either polypeptides or synthetic copolymers. This latter approach exhibits unique advantages. First, synthetic copolymers allow the direct extraction of membrane proteins from the cell membranes without requiring additional detergents that have been connected to proteins inactivation. Second, the ease of functionalization makes copolymer-nanodiscs highly customizable. For these reasons, recent advances in the development of nanodiscs-forming copolymers are attracting new attention to the field of membrane protein investigations. Chapter 1 intends to review membrane mimicking systems and the results of novel polymer modifications to overcome the current limitations and enhance polymer-based nanodiscs' applications to a broader variety of biophysical techniques used to study membrane proteins. Particular focus is given to the formulations developed in the Ramamoorthy research group.

Chapter 2 focuses on nuclear magnetic resonance (NMR) spectroscopy, a powerful technique that offers an atomic resolution. Unfortunately, its intrinsic low sensitivity results in long acquisition times that might exceed the sample's lifetime under investigation. Different paramagnetic agents have been used to decrease the spin-lattice (or $T_{1}$ ) relaxation times of the studied nuclei, which are the main cause for long acquisition times necessary for signal averaging to enhance the signal-to-noise ratio of NMR spectra. Consequently, most experimental time is "wasted" waiting for the magnetization to recover between successive scans. This chapter discusses how to set up an optimal paramagnetic relaxation enhancement (PRE) system to effectively reduce the $\mathrm{T}_{1}$ relaxation times, avoiding significant broadening of NMR signals.

Additionally, it describes how PRE-agents can be used to provide structural and dynamic information and can even be used to follow the intermediates of chemical reactions and speed-up data acquisition. Moreover, this chapter describes the unique challenges and the benefits associated with the application of PRE to solid-state NMR spectroscopy. A focus is given to explain how the use of PRE is more complex for membrane mimetic systems as PRE can also be exploited to change the alignment of oriented membrane systems. Furthermore, it is discussed how paramagnetic metals can be utilized further to increase the dynamic nuclear polarization (DNP) effects and preserve the enhancements when dissolution DNP is implemented. 
The PRE approaches involve introducing external paramagnetic probes in the system, which can induce undesired changes in the sample and the observed NMR spectra. For membrane proteins, it is possible to exploit PRE effects by (i) direct labeling with paramagnetic species on specific amino acid residue(s) in the membrane protein, (ii) using paramagnetically-labeled phospholipids, and (iii) using solvent PRE. Each of these ways offers both advantages and disadvantages, such as sample perturbations. Chapter 3 shows a fourth approach - SMA-EA-DOTA, a nanodiscs-forming SMAbased copolymer containing chelating units and able to host paramagnetic metals on the outer rim of the nanodiscs. This proposed modification shows a reduction in the $\mathrm{T}_{1}$ times with minimum-to-no alteration of the overall spectral quality in model systems. Among the advantages of using this fourth approach are (a) the retainment of the native-like lipid composition, (b) the absence of direct paramagnetic tags on the membrane protein, (c) the lack of unwanted solvent PRE that might cause significant peak broadening due to non-specific interactions between the investigated system and paramagnetic ions free in solution.

As mentioned, synthetic amphipathic copolymers are increasingly used to solubilize cell membranes directly and to reconstitute functional membrane proteins in native-like copolymer-lipid nanodiscs. However, the extraction requires large quantities of copolymers, and the use of paramagnetically labeled copolymers might be costly. In many studies reported in the literature, the right copolymer formulation's choice depends on many factors and experimental conditions, typically developed according to a trial-and-error process since each studied system requires adapted protocols. While the increasing number of nanodiscs-forming copolymers are reported to be useful, and they provide flexibilities in optimizing the sample preparation conditions, it is crucial to develop a systematic protocol that can be used for various applications. Nanodiscs-forming copolymers act, de facto, like «macromolecular detergents.» In this context, there is a vital necessity of benchmarking the performances of existing copolymer formulations, assessing crucial parameters for the successful extraction, isolation, and stabilization of membrane proteins. Chapter 4 presents a comparison among SMA-EA, anionic short-chained copolymer developed in our laboratory, and a set of anionic copolymer formulations commercially available under the names of XIRAN® SL25010 P, SL30010 P, SL40005 P. The reported results show how the critical micellar concentration (c.m.c.) of each copolymer is significantly altered in the presence of lipids and confirm the existence of an equilibrium between nanodiscs-bound and «free» or «micellar» copolymer chains in the solution. The speculation is that these findings can be exploited to optimize studies involving the necessity of special copolymers, which would simplify the applications and broaden the scope of polymer-based nanodiscs. 
Finally, Chapter 5 expands the findings discussed in Chapter 3 and introduces two new paramagnetically tagged SMA-based copolymers - ST-10 and ST-100. In place of the chelating units to host paramagnetic metals as previously discussed, ST-10 and ST-100 involve the insertion of spinlabels, i.e., stable organic free-radical paramagnetic species in the copolymer chains. This solution is intended to expand the applications of paramagnetically labeled copolymer-nanodiscs, for example as polarizing agents for DNP studies. Finally, Chapter 6 introduces the idea of using $\mathrm{Gd}^{3+}-$ and $\mathrm{Mn}^{2+}$ loaded SMA-EA-DOTA-nanodiscs as a macromolecular contrast agent for magnetic resonance imaging (MRI). 


\subsection{Biological membranes and membrane mimetics}

Biological membranes are vital components of any cell. They protect the organism from the external environment and provide functional barriers between subcellular compartments. Figure 1.1 shows an example of a eukaryotic cell membrane. The chemical scaffold is a phospholipid bilayer, but its composition is quite complex despite such an apparent structural simplicity ${ }^{1,2}$. In fact, different cells, and even organelles within the same cell, are made of various lipids (phospholipids, sphingolipids, and sterols). Their distribution can also be both symmetrical or asymmetrical in the two leaflets that constitute the bilayer ${ }^{3-5}$. As an example, mammalian cell membranes contain more than 1000 different phospholipids ${ }^{6}$.

The lipid bilayer is not only a semipermeable barrier, but it is involved in many biological processes. However, most properties ascribed to biological membranes cannot be explained merely considering the lipid moiety despite their crucial importance ${ }^{7}$. Among the essential components of the cellular membranes, membrane proteins are devoted to a plethora of cellular functions required for life, such as intercellular joining, enzymatic activity, transport (active/passive), cell-cell recognition, anchorage/attachment, signal transduction, etc. ${ }^{8-12}$

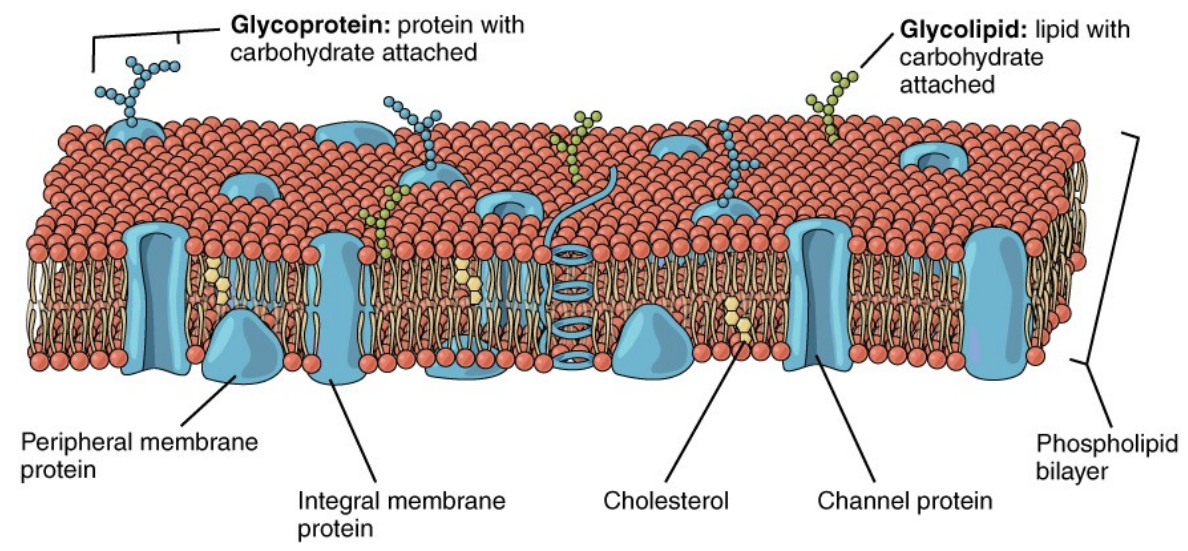

Figure 1.1. The cell membrane is a fluid mosaic model. The phospholipid bilayer comprises many different molecular components, including a variety of lipids, proteins, and cholesterol, some of which are with carbohydrate groups attached. This "fluid lipid sea" hosts other molecules such as membrane proteins ${ }^{1,2,13}$. This illustration is licensed under the Creative Commons Attribution 4.0 International ${ }^{14}$.

Membrane proteins are encoded in $\sim 30 \%$ of the human genome ${ }^{15}$, and they represent $\sim 60 \%$ of all drug targets ${ }^{16,17}$. Both academic and pharmaceutical research have focused on membrane proteins since, not surprisingly, their malfunctioning has been associated with numerous disease ${ }^{18}$. However, to date, only $\sim 2-3 \%$ of all protein structures reported in the Protein Data Bank (PDB) ${ }^{19}$ are membrane proteins, underlining how challenging this field of research is. 
The lack of membrane protein structural information limits our current understanding of cellular membrane biology ${ }^{20}$ and significantly hinders modern drug design ${ }^{21}$. Numerous challenges have plagued the study of functional membrane protein structures. Overexpression, extraction, and purification are all delicate steps in the production of membrane proteins sample. However, a crucial challenge in the structural biology investigation of membrane proteins is obtaining the sample's functional stability. Indeed, to be studied, membrane proteins must be extracted from the twodimensional solution they are in (i.e., the amphipathic lipid environment that constitutes the bilayer) to a three-dimensional solvent (i.e., buffer solutions). Unfortunately, due to their amphipathic nature, membrane proteins are not entirely soluble in aqueous solvents, thus the necessity of finding membrane mimicking systems able to host membrane proteins in their active form for the time necessary to perform the experiments ${ }^{22}$.

The functional and structural characterization of membrane proteins is a rapidly evolving field of research, thanks to technological advancements such as single-particle cryo-electron microscopy (cryo-EM). Among its advantages, cryo-EM does not require well-ordered single crystals for structural studies, and this is the primary reason for the increasing interest in this technology. However, as mentioned earlier, in optimal sample production, the challenges are still in place. Thus, the necessity of suitable membrane-mimicking systems ${ }^{23}$.

Membrane-mimetics are divided into non-bilayer systems, such as detergent micelles and amphipols, and bilayer systems, such as liposomes, supported lipid bilayers (SLBs), bicelles, and nanodiscs. The rest of this chapter provides a general overview of the various options, focusing on nanodiscs forming copolymers and polymer:lipid nanodiscs, the thesis's object.

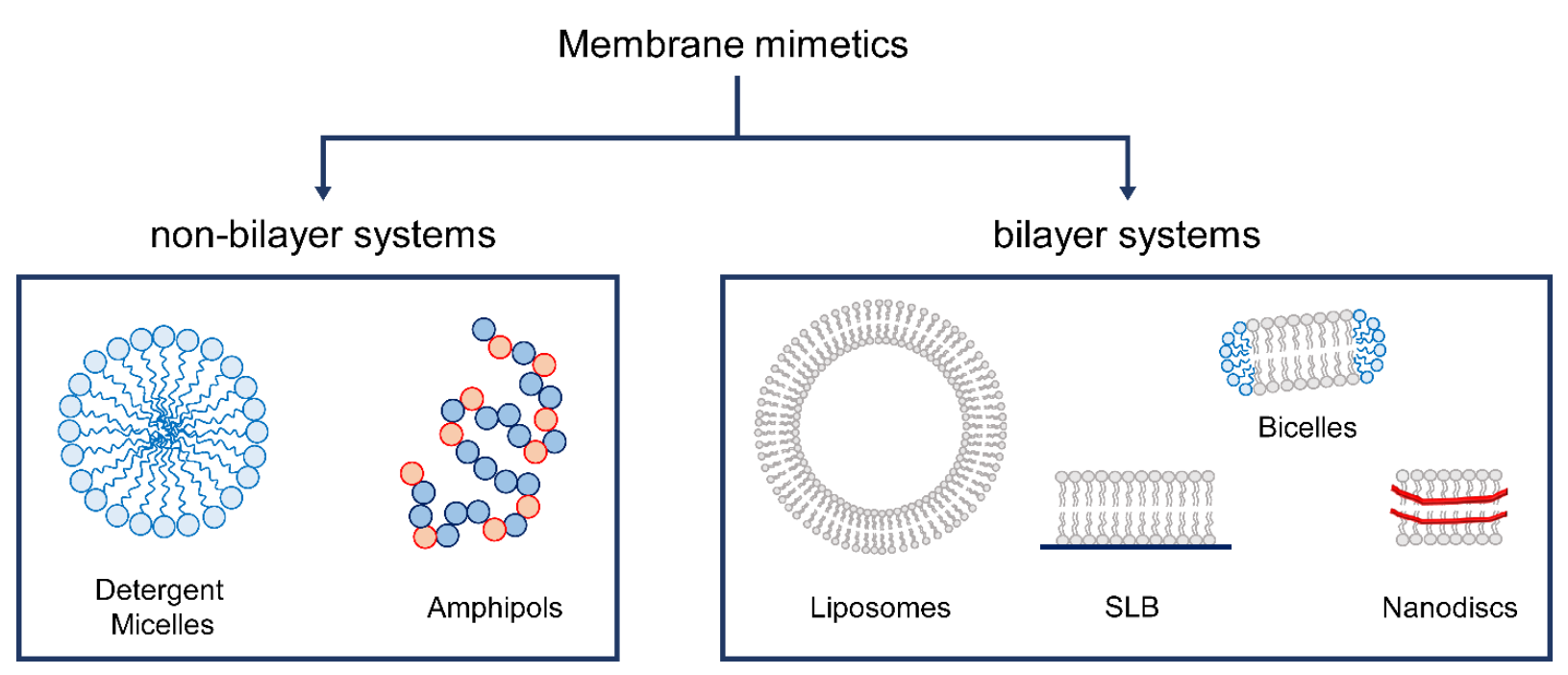

Figure 1.2. Classification of cell membrane mimetics. On the left non-bilayer model systems such as detergent micelles, while on the right are lipid bilayer model systems such as liposomes, SLBs, bicelles, and nanodiscs. 


\subsubsection{Detergents and detergent micelles}

One common method for membrane protein solubilization is the use of detergent micelles ${ }^{24,25}$ (Figure 1.3). Detergents are relatively small amphiphilic molecules that consist of long hydrophobic chains and a polar headgroup. This latter part is usually used to classify detergents in categories such as ionic (either positively or negatively charged), zwitterionic, and neutral. Figure 1.2 shows the chemical structures of some of the most used detergents per category. Figure 1.3 shows the supramolecular structure of detergent micelles in comparison with other model membrane-mimicking systems.

lonic detergents
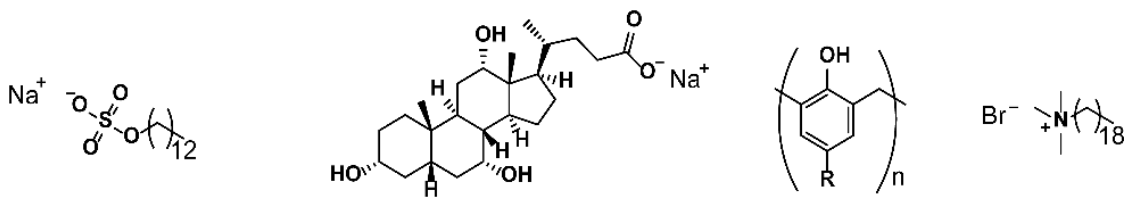

Sodium docecyl sulfate (SDS)

Sodium cholate

Calix[n]arene

CTAB

Zwitterionic detergents

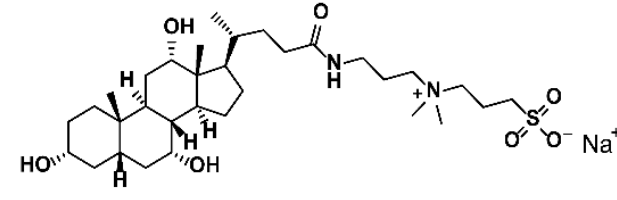

CHAPS

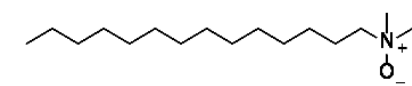

Lauryldimethylamine oxide (LDAO)

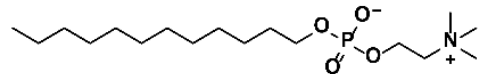

n-dodecylphosphocholine (DPC)

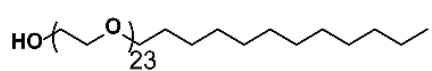

Polyoxyethylene lauryl ether

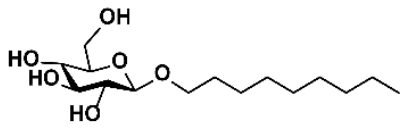

Non-ionic detergents
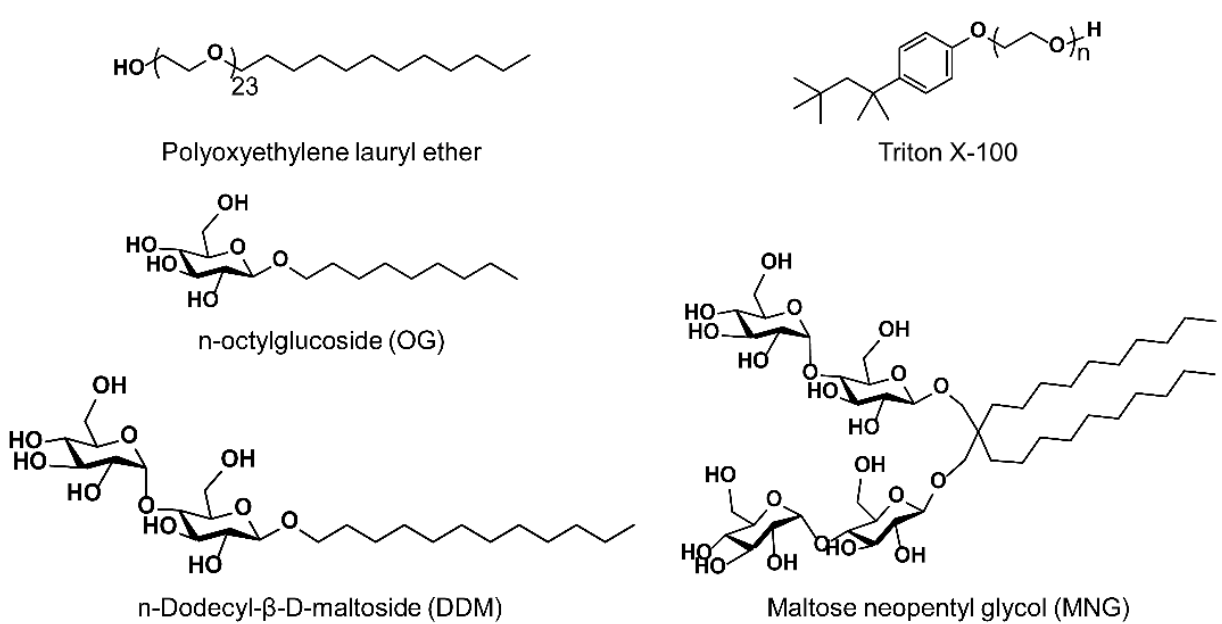

Figure 1.3. Molecular structures of widely employed detergents in the extraction of membrane proteins. Detergents are classified as ionic (either negatively or positively charged), zwitterionic, and non-ionic. A few examples of commonly used molecular systems for each of the categories are given.

Historically, detergents have been widely employed to extract, isolate, and purify membrane proteins from their native cellular environment ${ }^{26,27}$. However, detergents' membrane protein solubilization causes an undesirable perturbation of the native lipid bilayer, removing lipids that are tightly interacting with the membrane proteins and crucial for their function and structural integrity ${ }^{28-30}$. 
Moreover, the association of the detergent hydrophobic tails with the hydrophobic transmembrane region of the membrane protein cannot correctly mimic the bilayer environment. Although successful in many cases reported in the literature, this extraction and micelle formation commonly interferes with membrane protein folding, causing a loss in membrane protein native functionality ${ }^{31-35}$. Such a significant drawback greatly hinders the study of membrane proteins prompting the need for better membrane mimetics.

\subsubsection{Liposomes}

To circumvent the problems associated with the lack of a lipid bilayer, a common membrane mimetic used in membrane protein research is the liposome $e^{20,36}$. Liposomes are vesicles that consist of lipids that spontaneously assembled into a bilayer (e.g., DMPC, DPPC, POPC, DOPC, etc.) $)^{3}$. These are advantageous over detergents as the liposomal bilayer closely resembles the cellular membrane environment as compared to a detergent micelle ${ }^{36}$. Liposomes are classified based on parameters such as size and lamellarity. Figure 1.4 summarizes the main categories. Liposomes can be classified based on their size and lamellarity into multilamellar vesicles (MLVs), small unilamellar vesicles (SUVs), large unilamellar vesicles (LUVs), giant unilamellar vesicles (GUVs). The major drawback of liposomes as a membrane mimetic are (i) their relative instability, (ii) the limited use in various biophysical characterization techniques, and (iii) the need for detergent inclusion in the extraction process of membrane proteins ${ }^{37,38}$.

\subsubsection{Bicelles}

Another common membrane mimetic used to study membrane proteins is bilayer micelles, also known as bicelles ${ }^{39-42}$, disc-shaped phospholipid bilayers surrounded by a rim containing shortchained detergent molecules (Figure 1.3). The advantages of bicelles over liposomes are that bicelles have no membrane curvature, show higher stability and size tunability. Remarkably, the size tunability is achieved by changing the ratio of lipid to detergent (q-ratio) ${ }^{43}$.

By controlling the q-ratio, large bicelles $(q>2.5)$ have been shown to align in the presence of a magnetic field. These large bicelles are anisotropic as they do not tumble fast enough in the NMR time scale ${ }^{44}$. Bicelles have also been used for solution NMR studies when formed at lower sizes $(\mathrm{q}<$ 0.5 , also known as isotropic bicelles) $)^{45,46}$.

While advantageous over liposomes for NMR studies, bicelles still have the crucial problem of including detergents during the reconstitution of membrane proteins. The detergent molecules present 
in bicelles undergo diffusion from the rim to the planar lipid bilayer and lead to toroidal pores within the planar lipid bilayer of the bicelles ${ }^{47}$. The ability of the detergent molecule to diffuse into the lipid bilayer can denature an embedded protein ${ }^{20}$. To overcome these challenges, researchers have developed nanodiscs to better simulate a native-like membrane environment for membrane protein $\operatorname{research}^{48}$.
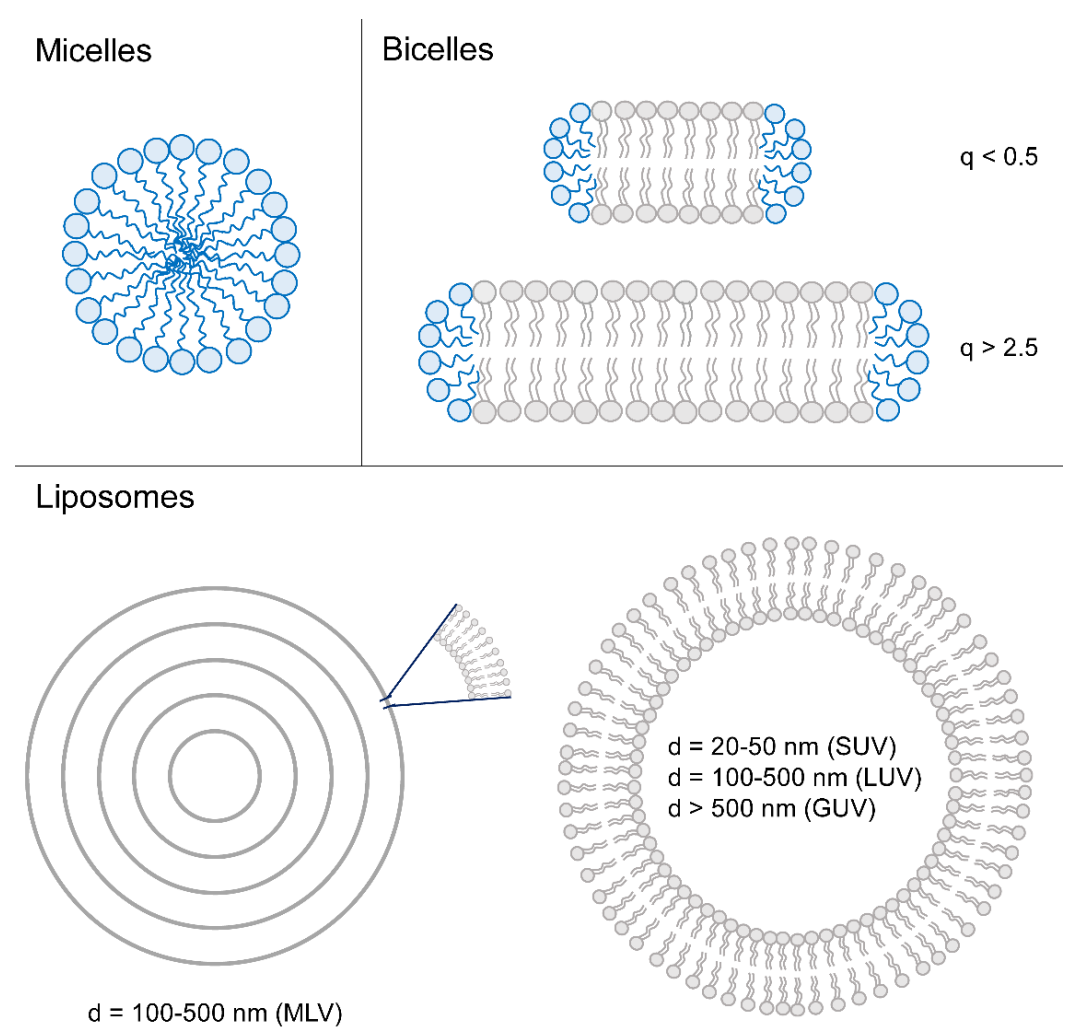

Figure 1.4. Supramolecular architecture of micelles, bicelles, and liposomes. The figure shows the commonly used membrane mimicking systems. For example, detergent or lipid micelles and isotropic bicelles that tumble sufficiently fast in the NMR time scale are frequently used in solution NMR studies whereas the large-size membrane mimetics such as large-size bicelles and liposomes/vesicles are commonly used in solid-state NMR applications. While all of these membrane mimetics are commonly used in the structural studies of membrane-associated peptides (including antimicrobial peptides, toxins, fusion peptides, amyloid peptides) and proteins, the lack of non-bilayer structure and curvature of micelles are capable of inducing undesirable effects. We recommend some of the review articles that comprehensively cover all aspects of these mimetics to the readers ${ }^{39,45,49-52}$. 


\subsubsection{Supported lipid bilayers (SLBs)}

SLBs are a cell-membrane-mimicking platform that consists of a lipid bilayer supported on a rigid substrate and can be used with surface-sensitive measurement techniques ${ }^{53}$. Used in imaging techniques, such as AFM, to mimic large flat lipid bilayers, they are also suitable for investigations on bilayers' mechanical properties and to mimic cell-cell junctions ${ }^{54}$.

\section{A}

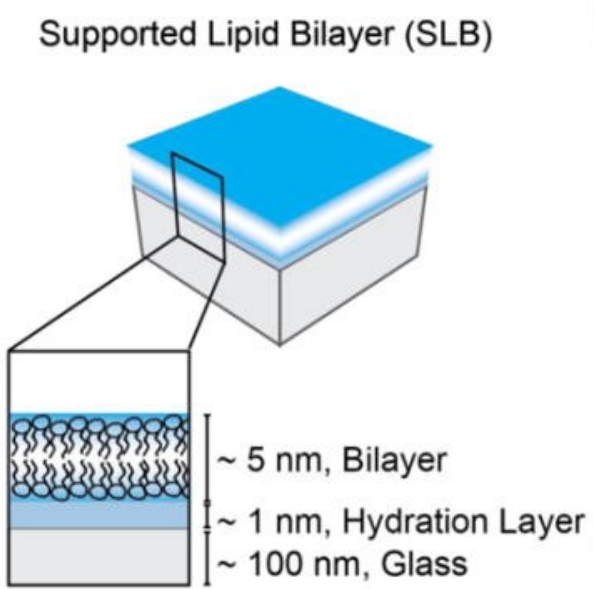

C

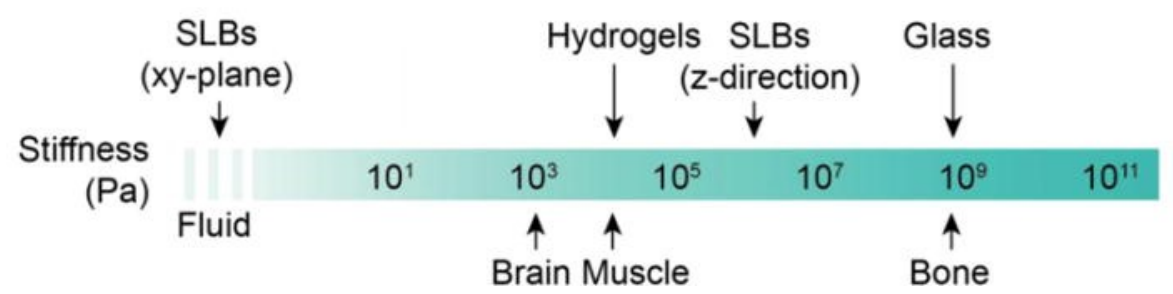

Figure 1.5. Supported Lipid Bilayer (SLB) design and mechanics. (A) SLBs contain a bilayer separated from a rigid substrate by a thin layer of water. (B) Representative FRAP of labeled lipids illustrating SLB lateral fluidity. Lipids in SLBs freely diffuse within the plane on three representative substrates. Following photobleaching, diffusion causes photobleached lipids to be diluted, and the average fluorescence to increase. The disappearance of a visible bleached region indicates total recovery and a fluid bilayer. (C) SLB stiffness in comparison to tissue, hydrogels, and glass substrates. SLBs are anisotropic, behaving like fluids in the XY-plane, but stiffer than hydrogels in the Z-direction. The figure is used with permission from ${ }^{54}$. Copyright 2017 Elsevier. 


\subsubsection{Amphipols}

Amphipols (APols) are amphipathic polymers carrying many hydrophilic and hydrophobic groups. They show various chain lengths and charge densities, constituting a class of surfactants known as "polysoaps" particles due to the favorable combination of a short backbone chain length and an appropriate number and size of hydrophobic/hydrophilic segments, flexibility, and a limited dispersity. APols interact with the transmembrane regions of membrane proteins, solubilizing and stabilizing them ${ }^{56}$. The chemistry of APols offers rich opportunities for labeling and functionalization, opening to a plethora of different solutions. The most employed amphipol for reconstituting membrane proteins is A8-35. Amphipols have been used to reconstitute and determine high-resolution cryo-EM structures of various membrane proteins: capsaicin receptor; transient receptor potential non-selective cation channel (TRPV1) ${ }^{57}, \gamma$-secretase ${ }^{58}$, V-type ATPase ${ }^{59}$, human polycystin-2 (PC2) ${ }^{60}$.

a

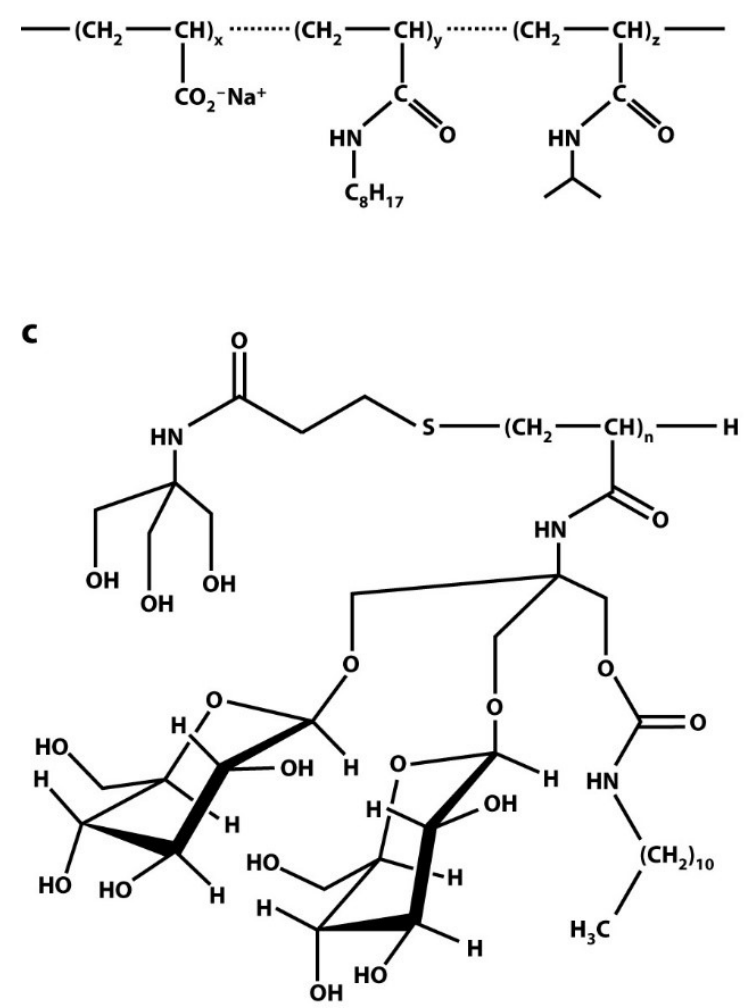

b

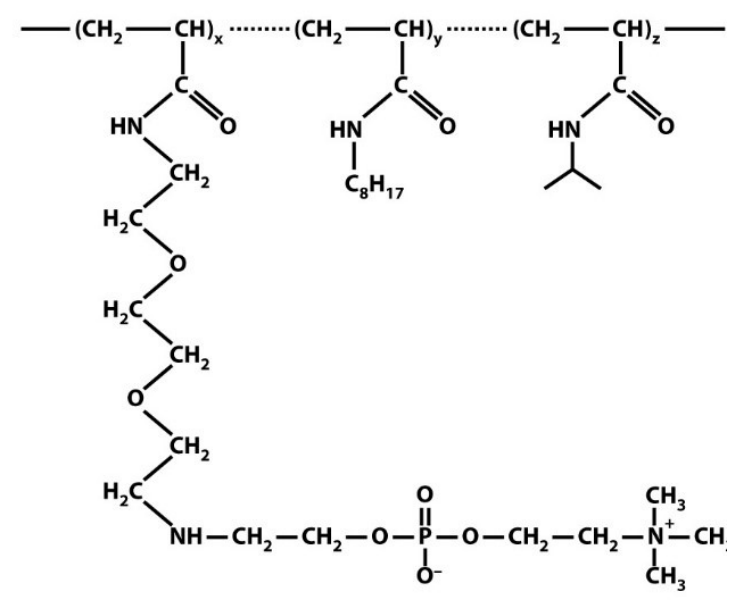

d

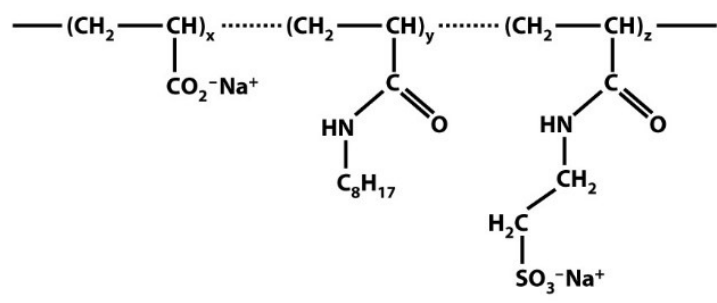

א Popot J-L, et al. 2011.

Annu. Rev. Biophys. 40:379-408

Figure 1.6. Chemical structures of four types of amphipols. (a) A polyacrylate-based APol, A8-35 (87); (b) a phosphorylcholine-based APol, C22-43 (26); (c) a nonionic, glucose-based APol (71); and (d) a sulfonated APol (23). The figure was used with permission from ${ }^{61}$. Copyright 2011 Annual Reviews. 


\subsubsection{MSP-Nanodiscs, Salipro, peptidiscs, and copolymer-nanodiscs}

Introduced by Sligar and coworkers in 2002, nanodiscs are noncovalent nanoparticles that consist of a disc-shaped lipid bilayer stabilized by an amphiphilic membrane scaffold protein (MSP) ${ }^{62-64}$, inspired from high-density lipoproteins (HDL) ${ }^{65}$. MSP nanodiscs can be made by mixing MSPs with detergent-solubilized protein and lipids ${ }^{48}$.

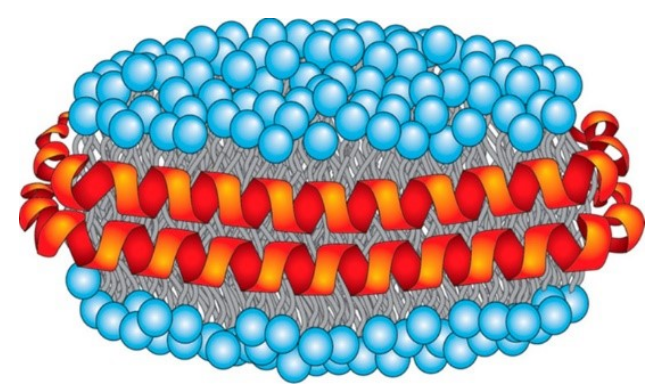

Figure 1.7. Schematic representation of MSP-Nanodiscs. Nanodiscs are discoidal lipid bilayer stabilized by encircling amphipathic helical scaffold proteins termed as membrane scaffold protein (MSP). Reproduced with permission from ${ }^{52}$. Copyright 2014 CRC Press.

Scaffold protein nanodiscs have been very successful in the study of several membrane proteins $^{52,63,65-69}$ and amyloid proteins ${ }^{70,71}$. The curvature-free nanodiscs have been demonstrated to be highly valuable to understand amyloid aggregation mechanism and to trap amyloid oligomers for high-resolution NMR based structural studies ${ }^{70}$. However, incorporating proteins into MSP nanodiscs still requires the use of detergents during the reconstitution process ${ }^{48}$.

MSP based nanodiscs also introduce some interfering properties during the study of reconstituted membrane proteins. Even though very recent studies reported the possibility of increasing the size of MSP-based nanodiscs ${ }^{67}$, which are otherwise small (typically $<15 \mathrm{~nm}$ diameter), there are difficulties in reconstituting large-size membrane proteins or protein-protein complexes. Some of these difficulties have been overcome using short amphipathic peptides engineered from the MSP $\operatorname{protein}^{72,73}$. These peptides have been shown to self-assemble with lipids to form nanodiscs and enable the reconstitution of protein-protein complexes. These nanodiscs undergo collision and exchange lipid contents, as demonstrated by a recent study using high-speed AFM and ${ }^{31} \mathrm{P}$ NMR experiments ${ }^{74-76}$. The peptide-based nanodiscs are useful for structural studies of membrane proteins using solution $\mathrm{NMR}^{72}$, $\mathrm{SAXS}^{77}$, solid-state $\mathrm{NMR}^{78}$, and also used for potential cancer immunotherapy ${ }^{79,80}$.

To expand the applicability of MSP nanodiscs, a diverse pool of MSP variants has been developed ${ }^{52}$. While peptide-based nanodiscs are increasingly utilized, their interference with biophysical studies of the embedded protein of interest and other possible undesired effects for in-vivo applications are 
inherent limitations for further biological and biomedical applications. Therefore, to overcome these limitations, there is a significant interest in developing different types of amphipathic molecules that can form nanodiscs. For completeness, two peptide-based alternatives to MSP and derivatives must be mentioned - saposin-lipoprotein (Salipro) ${ }^{81}$ and Peptidiscs. Saposins are small membrane-active proteins and can be used for the reconstitution of membrane proteins such as the bacterial peptide transporter PeptT $\mathrm{T}_{\mathrm{So}}$, investigated via cryo-EM ${ }^{82}$. Peptidiscs, unlike the MSP-based nanodiscs, only requires a short amphipathic bi-helical peptide $\left(\mathrm{NSP}_{\mathrm{r}}\right)$ and no extra lipids, opening to detergent-free peptide-based MPs solubilization ${ }^{83-85}$. Due to their amphipathic properties, in the presence of bilayerforming lipids, SMA and other copolymers form discoidal lipoparticles analogs to MSP-nanodiscs. Such a promising technology has been successfully used for isolation, purification, structural and functional characterization of membrane proteins ${ }^{86,87}$ and detailed in the next paragraphs. 


\subsubsection{Styrene-maleic acid lipoparticles and copolymer-nanodiscs}

Poly(styrene-co-maleic acid), SMA, is an amphiphilic hypercoiling copolymer obtained by hydrolyzation of the poly(styrene-co-maleic anhydride), SMAnh ${ }^{86,88,89}$. In the 1990s, Seki et al. found that in the presence of bilayer-forming lipids, SMA acts as a polymeric "detergent," capable of lysing DPPC vesicles. By the end of the decade, it was noted that hypercoiling copolymers such as SMA were able to produce lipid-polymer nanostructures analogous to lipoprotein assemblies such as highdensity lipoproteins nanoparticles (HDL) ${ }^{90}$.

In 2009, styrene-maleic acid lipoparticles (SMALPs), or polymer nanodiscs, were employed for the first time to study membrane proteins ${ }^{91}$. The molecular architecture of SMALP resembles MSPnanodiscs - lipid bilayer patches surrounded by an amphipathic belt. Still, the main advantage of using SMA copolymer is the possibility of direct extraction of membrane proteins from their native environment while retaining the native lipid membrane composition. Most importantly, this extraction and isolation process is accomplished without the use of any destabilizing detergents ${ }^{92-94}$. Since the report dated May 2009, several groups adopted SMALPs in their research. Figure 1.8 below shows the exponential growth of the field.

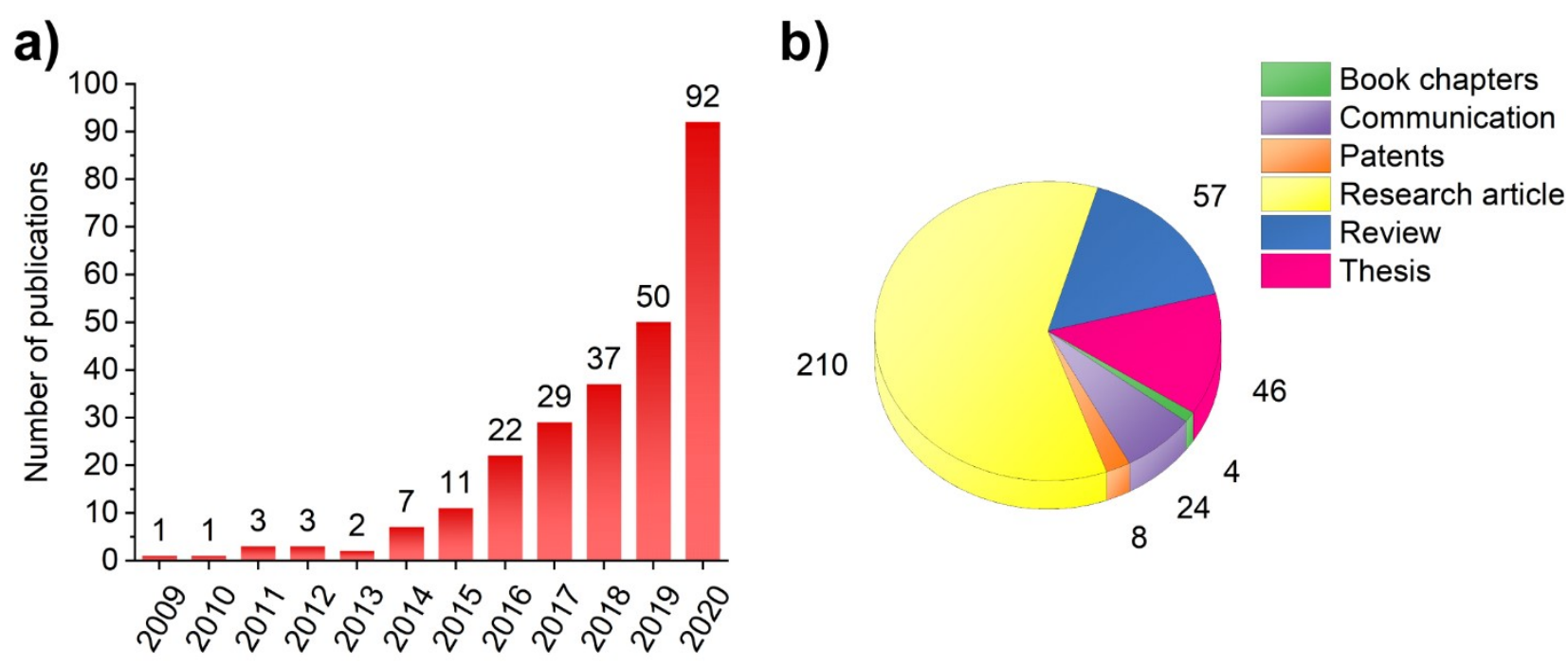

Figure 1.8. SMALP: a growing community. a) shows the number of peer-reviewed publications per year since the first publication on SMALP appeared in May 2009. b) shows the type of publication. Since the first article, 8 patents have been deposited, 24 communications and 210 research articles have been published. Also, 46 students received their Ph.D. with dissertations on a topic that uses SMALP as a tool to investigate membrane proteins. Notably, due to the multidisciplinary nature of the field, there have been 57 review articles in the span of 10 years. Both graphs are updated until 31 December 2020. More details are available at http://www.smalp.net. 


\subsubsection{SMAs copolymers}

The copolymerization of styrene and maleic anhydride has been known since the 1940s and has been one of the most extensively investigated reactions due to the strong tendency to form alternating copolymers $^{95}$. Obtained by free-radical polymerization, SMAnh is a thermoplastic copolymer with a high glass transition temperature, good mechanical properties (i.e., good impact, rigidity, and dimensional stability), high heat and chemical resistance. Since the 1960s, high-molecular-weight variants of SMAnh copolymer found wide-ranging applications from molding resin in the automotive, packaging, and construction industries to compatibilizer in plastic blends and alloys to improve the overall properties ${ }^{90,96}$.

In the 1980s, Maeda and coworkers demonstrated that SMA copolymer significantly improved the physicochemical, biochemical, and pharmacological properties of neocarzinostatin (NCS) ${ }^{97}$. Because of its macromolecular nature, the resulting conjugate SMANCS exhibited desirable features such as prolonged plasma half-life, pronounced tumor targeting efficiency, and lower acute toxicity in rats and mice ${ }^{98}$. Also, micellar nanoparticles of SMA have been proposed as drug carriers for tumortargeting with reduced general toxicity ${ }^{99-101}$.

Most of the recent SMA research applied to structural biology has involved investigating several different polymer formulations. The simple variation of molecular weights and styrene to maleic anhydride ratios leads to various commercially available polymers ${ }^{102,103}$. For membrane proteins investigations, small-molecular weight SMAs are used. With a variable molecular weight range (2$10 \mathrm{kDa}$ ) and a styrene-to-maleic anhydride ratio that varies from 1:1 to 2:1 and 3:1 (Figure 1.9), various formulations of SMAs were used to extract and isolate membrane proteins directly ${ }^{91,104-118}$.

$1: 1$

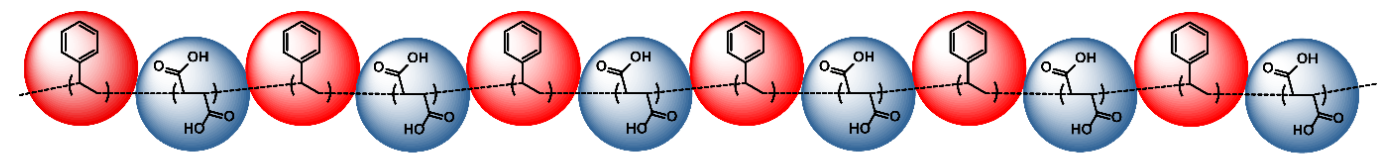

$2: 1$

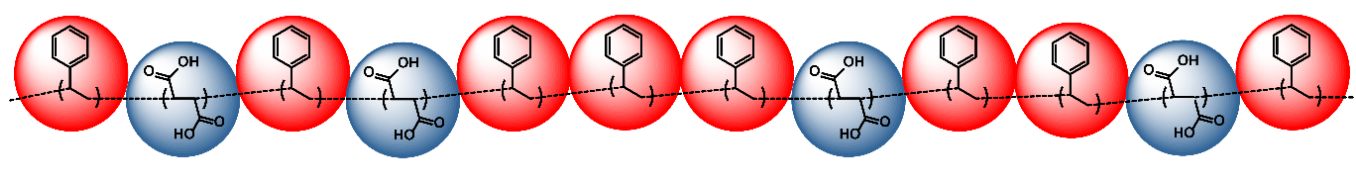

$3: 1$

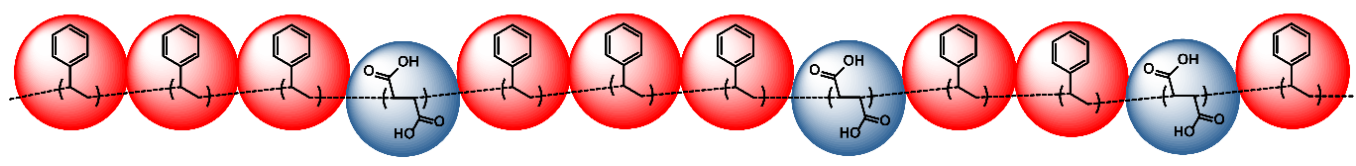

Figure 1.9. SMAs' most common formulations. SMA has been used in the styrene-to-maleic acid ratio that goes from 1:1 to 3:1 ratio of styrene:maleic acid. The advantages over micelles, liposomes, bicelles, and protein-based nanodiscs allow polymer nanodiscs to be applied to a broader variety of membrane protein studies. 


\subsubsection{Formation of SMA lipoparticles}

The ability to solubilize lipids without the necessity for detergents, transforming lipid bilayers into stable nanodiscs, is among the most significant advantages of SMA. This method preserves native interactions of membrane proteins with lipid species, offering a better membrane mimicking system. Still, the formation mechanism of the polymer nanodiscs is not fully understood. Scheidelaar et al. ${ }^{119}$ proposed a three-step model for the solubilization of lipid membranes by SMA copolymers (Figure 1.10). First, SMA binds to the hydrophilic surface of the bilayer. Then, it inserts its hydrophobic moiety into the hydrophobic core of the bilayer, driven by the hydrophobic effect. Finally, the destabilized bilayer evolves into polymer-lipid discoidal patches or SMA lipoparticles (SMALPs).
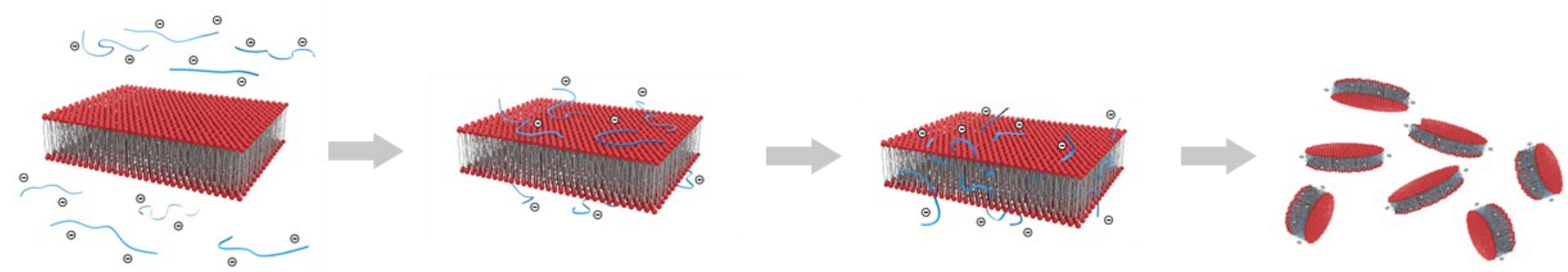

Figure 1.10. An illustration of a three-step model for the formation of SMA based lipid-nanodiscs. Step 1. Electrostatic interactions between the surface of the lipid bilayer and the copolymer chains. Step 2. Insertion of the styrene moieties in the hydrophobic core of the lipid bilayer, driven by the hydrophobic effect. Step 3. The destabilized bilayer evolves into nanodiscs because of the electrostatic repulsion of the hydrophilic maleic acid moieties of the copolymer.

The kinetics of the solubilization is a process modulated by the lipids' packing and bilayer thickness. Thus, the speculation is that the rate-determining step is the second. In most cases, solubilization can be improved by increasing temperature or by freeze-thaw cycles. It is observed that SMA induces maximal solubilization of bilayers at a temperature beyond the melting temperature of the lipids (i.e., lipids in the liquid-crystalline phase) for saturated lipids. In contrast, unsaturated lipids were more challenging to solubilize than saturated lipids in the fluid phase. The explanation can be related to the increased lateral pressure in the acyl-chain region, leading to a less efficient insertion of the polymers ${ }^{120,121}$. Besides, copolymer chain lengths, $\mathrm{pH}$, and ionic strength are two factors that influence SMA solubilization of membranes ${ }^{88,122}$. SMA's efficiency is $\mathrm{pH}$-dependent and is slower but overall more efficient at high $\mathrm{pH}$ values ${ }^{88,120,123-125}$

To shed light on the solubilization process, SMALP formation, and its supramolecular structure, MD simulations have been used. Although complete solubilization was not observed on the timescale of their CG MD simulations, Xue et al. ${ }^{126}$ findings seem to confirm the three-step model. In particular, the simulations predicted the growth of transmembrane pores due to the insertion of SMA copolymer 
chains as a crucial step in the membrane destabilization before the formation of SMALPs ${ }^{126}$. OrwickRydmark et al. ${ }^{127}$ observed the appearance of pores in the bilayer before the evolution into nanodiscs.

\subsubsection{SMALPs as membrane mimetics}

SMA gained interest in the field of membrane research because of the ability to form lipid nanodiscs, i.e., copolymer-lipid nanoparticles made of bilayer patches surrounded by copolymer chains act as an amphipathic belt, according to an HDL-like architecture (Figure 1.7).

Although the copolymer hypercoiling properties and membrane solubilization capacity are known to be affected by $\mathrm{pH}$ and ionic strength, the hydrodynamic diameters of SMALPs are independent of ionic strength ${ }^{123,128}$ and not related to $\mathrm{pH}$ values. Among the advantages of using SMAs is that the bilayer environment reflects the lipids' organization in the native membrane, and it does not show any lipids preference ${ }^{129}$. However, it has also been found that protein-containing SMALPs isolated from native membranes are enriched in certain lipid species as compared with protein-free SMALPs ${ }^{106,117}$. Reports show that the lipids incorporated into SMALPs do not show a highly cooperative phase transition from gel to liquid-crystalline phase, in contrast with MSP nanodiscs ${ }^{130-132}$.
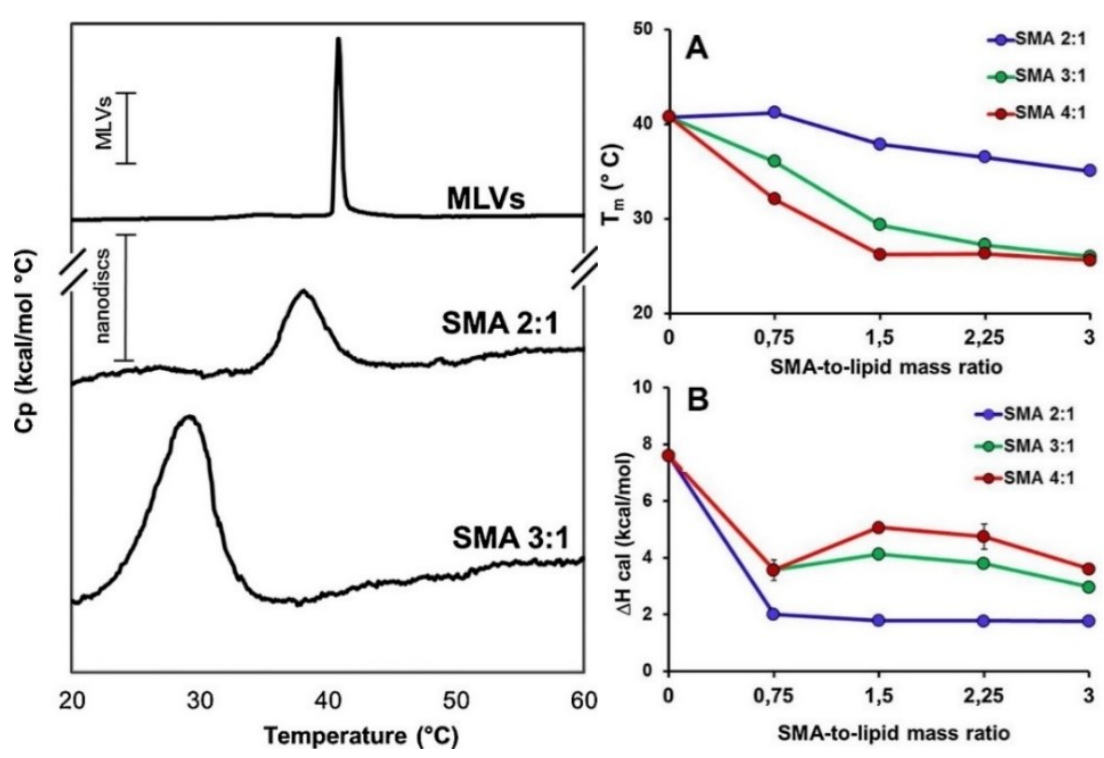

Figure 1.11. Representative differential scanning calorimetry (DSC) thermograms of di-16:0 PC lipids selfassembled in MLVs, SMA 2:1 nanodiscs and SMA 3:1 nanodiscs (left panel). Nanodiscs were obtained at a SMA-tolipid mass ratio of $\sim 1.7$. The inserted scale bar at the top (MLVs) corresponds to $5 \mathrm{kcal} / \mathrm{mol}{ }^{\circ} \mathrm{C}$ while the bar at the bottom (nanodiscs) corresponds to $0.5 \mathrm{kcal} / \mathrm{mol}{ }^{\circ} \mathrm{C}$. (Right panel) Variation in A) $\mathrm{T}_{\mathrm{m}}$ and $\mathrm{B}$ ) $\Delta \mathrm{H}_{\text {cal }}$ of the gel-to-fluid phase transition of di-16:0 PC lipids in nanodiscs bounded by SMA 2:1 (blue circles) SMA 3:1 (green circles) or SMA 4:1 (red circles). Data are given as averages obtained from the 2 nd and 3 rd heating cycle from 2 independent samples. Errors representing the standard deviation are covered by the markers. The figure is reproduced with permission from ${ }^{133}$. Copyright 2017 Elsevier. 
In fact, SMA-nanodiscs show a broad non-cooperative thermotropic phase transition, often anticipated by a pre-transition. It was also found that more hydrophobic formulations such as SMA 3:1 $(\sim 9.5 \mathrm{kDa})$ have shown to exert lateral pressure on the lipid tails without perturbing the center (terminal methyl groups $)^{130}$. SMA 2:1 ( 9.5 kDa) better preserves the lipid packing properties in the nanodiscs if compared to SMA 3:1 $(\sim 9.5 \mathrm{kDa})$, as it is observed in DSC thermograms ${ }^{134}$, and present slower lipid exchange than the 3:1 counterpart, still faster than MSP nanodiscs or liposomes ${ }^{123}$. SMALPs are highly dynamic colloids that readily exchange membrane proteins and lipids ${ }^{128,135}$. The hypothesis is that SMALPs quickly exchange lipids and that any enrichment must result from preferential protein/lipid interactions preserved in SMALPs ${ }^{136}$. Although SMALPs keep a lipidbilayer core, they appear to be more dynamic than other membrane mimics ${ }^{123,128,135}$. In fact, SMALPs can be described more according to a highly dynamic equilibrium rather than kinetically trapped membrane mimetics ${ }^{136}$. Thus, lipids composition in membrane proteins containing nanodiscs is not merely a "snapshot" of the situation in the original membrane at the time of solubilization but reflects relatively strong protein/lipid interactions ${ }^{136}$.

A similar situation is expected for copolymer chains in the outer rim of copolymer-nanodiscs. This aspect will be further discussed in Chapter 4.

\subsubsection{Improving SMA copolymers}

The most widely used synthetic method to obtain commercial SMAs uses the conventional radical copolymerization in a continuous stirred tank reactor $(\mathrm{CSTR})^{137}$. This technology allows narrow chemical composition distribution (i.e., in terms of styrene-to-maleic anhydride units) but inevitably produces dispersity of chain lengths $(Đ>2)^{138-140}$. All synthetic copolymers show dispersity, and SMA is not an exception. However, to reduce $Đ$ in chain lengths, it is possible to use the polymerization mediated by reversible addition-fragmentation chain transfer (RAFT) ${ }^{141-144}$. Unfortunately, this method allows less control in the chemical composition (particularly for non-1:1 styrene-to-maleic molar ratios), causing a comonomer gradient along the chain, and the presence of blocks of polystyrene, due to the high tendency of styrene monomers to homopolymerize ${ }^{138}$.

A recent study suggests that RAFT-produced SMA copolymers are less efficient than their polydisperse (CSTR) commercially equivalent ones in solubilizing E.coli membranes ${ }^{145}$. However, other literature results show comparable performances between CSTR-SMA and RAFT-SMA in terms of nanoparticles' sizes, membranes dissolution capabilities, and the ability to extract membrane proteins $^{140,146}$. Although unwanted because it introduces undesired complexity to the samples, speculations assert that copolymers' dispersity might play an important role in destabilizing 
membranes, promoting nanodiscs ${ }^{88,120,121}$. Clearly, more evidence is needed to clearly state whether the use of RAFT-produced copolymers is more advantageous.

a)

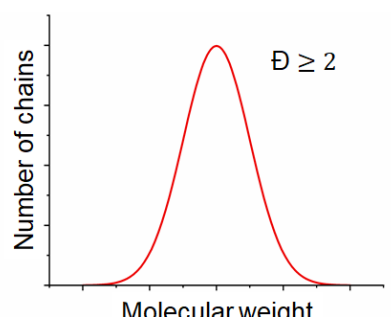

Molecular weight

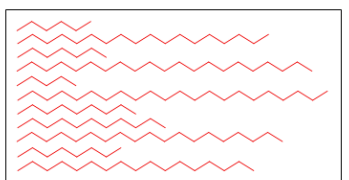

b)

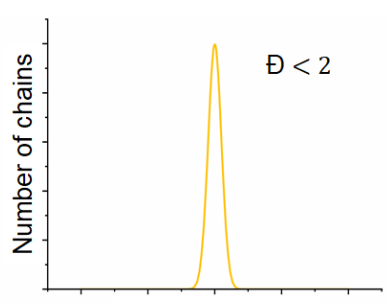

Molecular weight

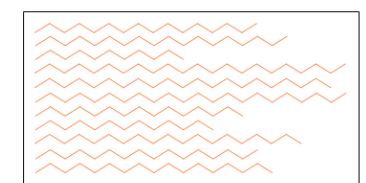

c)

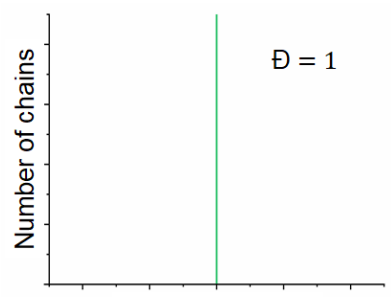

Molecular weight

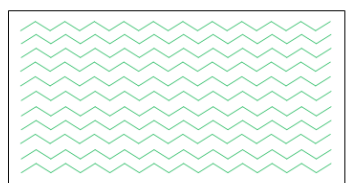

Figure 1.12. Dispersity of polymers. Regardless of their composition, synthetic polymers are dispersed. Dispersity, defined as $Đ=M_{w} / M_{n}$, shows how the term "polymer" refers to a plethora of macromolecules with different molecular weights distribution. c) denotes the ideal case, typical of small molecules. b) refers to narrow molecular weight distribution, while a) simulates the actual synthetic polymers. The larger the value of $Đ$ is, the more disperse is the polymer sample.

Among the advantages of using synthetic polymers is the possibility of customization ${ }^{96,103,147,148}$. For example, the maleic anhydride units of SMAnh copolymers react with primary amines via a nucleophilic ring-opening reaction ${ }^{149}$, allowing the modification of the hydrophilic moiety (Figures 13a-b). The hydrophobic moieties can also be changed, offering the possibility of tailoring the final product toward specific wanted applications. For example, Figure 1.13c shows how the styrene moiety can be substituted by introducing new hydrophobic groups such as diisobutyl (e.g., DIBMA $^{150}$ ) or stilbene (e.g., STMA ${ }^{151}$ ). 
a)

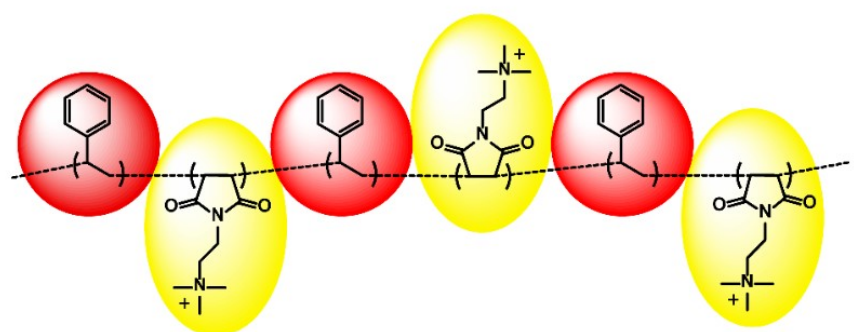

b)

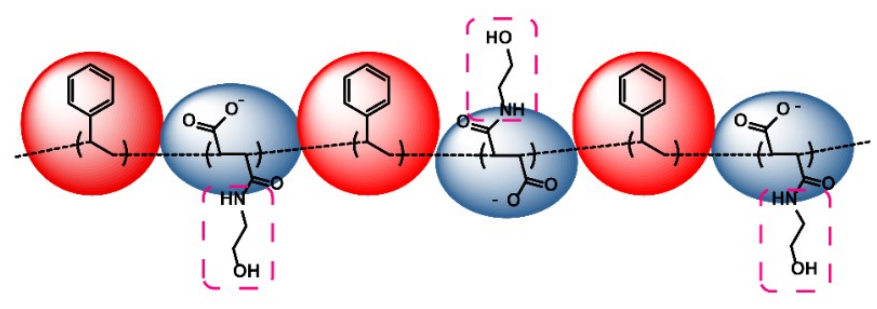

c)

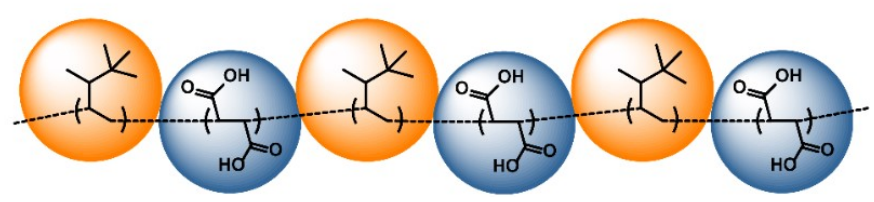

Figure 1.13. Variants of functionalization of the copolymeric backbone. The maleic acid moiety can be "substituted" by maleimide rings (a) (e.g., SMI ${ }^{152}$, SMA-QA ${ }^{153}$ ) or functionalized with pending groups (b) achieving different charge sign and densities (e.g., zSMA ${ }^{154}$, SMAd-A ${ }^{155}$, SMA-EA ${ }^{156}$, SMA-ED ${ }^{155}$, SMA-SH ${ }^{157}$, etc.). In addition, the styrene moiety might be replaced with other hydrophobic groups such as diisobuthyl groups or other alchil- and aryl- chains (e.g., DIBMA $^{150}$, PAAs $^{132}$, PMAs $^{158}$, STMA $^{151}$ ).

The first SMA copolymers used to form SMALPs $(\sim 7-9.5 \mathrm{kDa})$ led the way toward detergent-free extraction and isolation of membrane proteins but showed some limitations. For example, early SMAs did not allow for any significant size control over the nanodiscs ${ }^{89}$. MSP-based and copolymer nanodiscs are of about 10-20 nm diameter. Larger sizes would be preferred to host large MPs or large complexes. Moreover, all SMA based polymer nanodiscs are unstable to conditions that require low $\mathrm{pH}$ values or the presence of divalent metal ions ${ }^{88,92}$. The instability of SMA is due to the presence of carboxylic acids as the hydrophilic portion of the polymer. SMA is only soluble in water at $\mathrm{pH}$ values that are high enough to allow for carboxylate groups. Besides, carboxylate groups strongly bind divalent metal ions, forming chelates and leading to the precipitation of SMAs ${ }^{88,92}$. These limitations have restricted the applications of SMA-based nanodiscs. They do not allow for the active study of membrane proteins whose function requires low $\mathrm{pH}$ or the presence of divalent metal ions such as $\mathrm{Ca}^{2+}$ or $\mathrm{Mg}^{2+159,160}$. To overcome these limitations and expand SMAs' applicability, a variety of polymers has been proposed. The Ramamoorthy research group has focused on the hydrophilic functionalization of SMA (Figure 13a-b), employing a lower molecular weight SMA as our starting material. This choice allowed size control and improved the tolerance of SMALPs toward low $\mathrm{pH}$ values and a higher concentration of divalent cations. The following Table 1 contains a list of SMA- 
based copolymers able to form lipid-nanodiscs and summarizes some of the copolymers' most relevant features, whose structure is shown in Figure 1.14.

\begin{tabular}{lccccccc}
$\begin{array}{l}\text { Copolymer } \\
\text { name }\end{array}$ & Subunit ratio & $\begin{array}{l}\text { Charge } \\
\text { (at neutral pH) }\end{array}$ & $\begin{array}{l}\text { Molecular weight of } \\
\text { the starting material }\end{array}$ & $\begin{array}{c}\text { Reaction } \\
\text { method }\end{array}$ & Year & Reference \\
\hline SMA & $3: 1,2: 1$ & Negative & 9,500 & 3,050 & CSTR & 2009 & 161 \\
SMA-SH & $2: 1$ & Negative & 7,500 & N/A & CSTR & 2016 & 157 \\
zSMA & $1: 1$ & Zwitterionic & $>10,000$ & $>30,000$ & RAFT & 2017 & 154 \\
ePSMA & N/A & Negative & N/A & N/A & CSTR & 2017 & 162 \\
SMA-EA & $1.3: 1$ & Negative & N/A & 1,600 & CSTR & 2017 & 156 \\
SMA-ED & $1.3: 1$ & Zwitterionic & N/A & 1,600 & CSTR & 2017 & 155 \\
SMAd-A & $1.3: 1$ & Positive & N/A & 1,600 & CSTR & 2017 & 155 \\
SMA-QA & $1.3: 1$ & Positive & N/A & 1,600 & CSTR & 2018 & 153 \\
SMI & $2: 1$ & Positive & 7,500 & 2,700 & CSTR & 2018 & 152 \\
SMA-EA-DOTA & $1.3: 1$ & Negative & N/A & 1,600 & CSTR & 2019 & 163 \\
SMA-Pos & $2: 1$ & Positive & N/A & $>10,000$ & RAFT & 2020 & 164 \\
SMA-Neut & $2: 1$ & Zwitterionic & N/A & 6,900 & RAFT & 2020 & 164 \\
SMA-Glu & $2: 1$ & Negative & N/A & 42,100 & RAFT & 2020 & 164 \\
ST-10 & $1.3: 1$ & Negative & N/A & 1,600 & CSTR & 2020 & Unpubl.* \\
ST-100 & $1.3: 1$ & Negative & N/A & 1,600 & CSTR & 2020 & Unpubl.*
\end{tabular}

Table 1.1. List of SMA-based copolymers. A list of SMA-based copolymers is shown in chronological order, from the first appearance of SMA to extract membrane proteins to the latest. The table does not show RAFT variants of CSTR synthesized copolymers and does not include formulations showing different styrene-to-maleic acid ratios. ST-10 and ST-100 will be discussed in Chapter 5. 


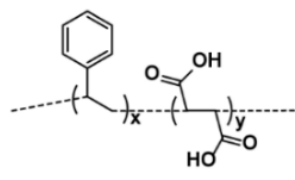

SMA*

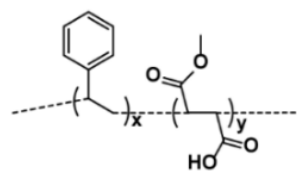

EPSMA

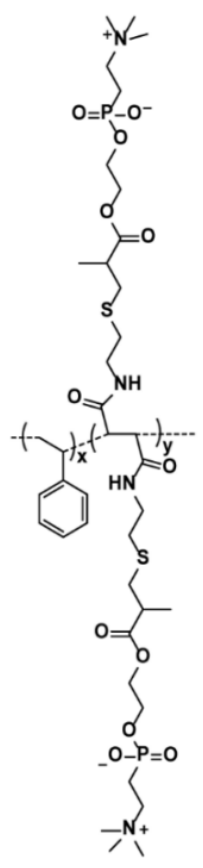

zSMA

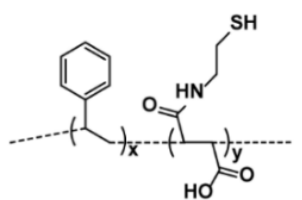

SMA-SH

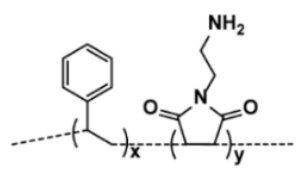

SMAd-A

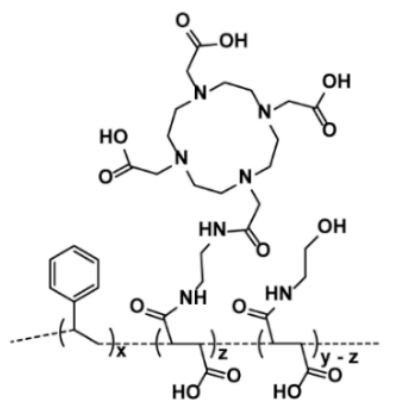

SMA-EA-DOTA *

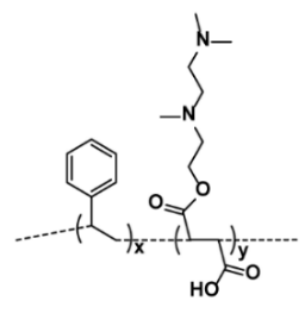

SMA-Pos

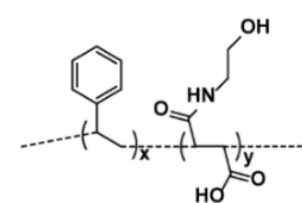

SMA-EA *

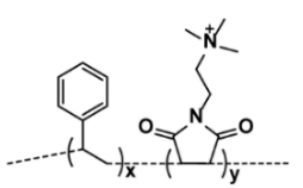

SMA-QA

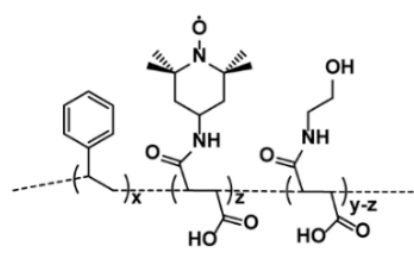

ST-10*

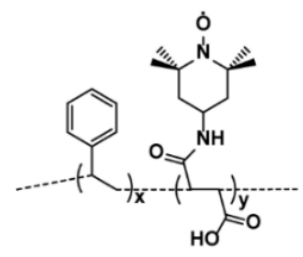

ST -100 *

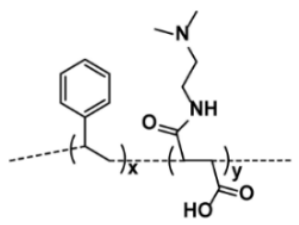

SMA-Neut

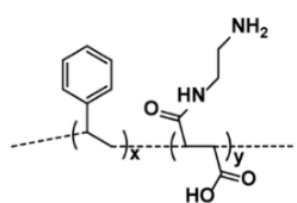

SMA-ED

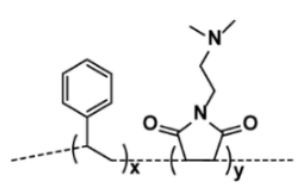

SMI

Figure 1.14. Chemical structures of SMA copolymer and its derivatives. In the past 11 years, a variety of formulation has been synthesized. Because of the maleic anhydride ring's reactivity, post-polymerization modifications were developed to expand the applicability of SMAs. Compared to SMA copolymer with equal styrene-to-maleic anhydride/acid moieties, SMA-SH ${ }^{157}$, SMA-EA ${ }^{156}$, and SMA-Glu ${ }^{164}$ formulations enable the perturbation of the charge density in the copolymer chains. SMAd-A ${ }^{155}, \mathrm{SMA} \mathrm{ED}^{155}, \mathrm{zSMA}^{154}$, and SMA-Neut ${ }^{164}$ are zwitterionic at neutral $\mathrm{pH}$ conditions. SMA-QA ${ }^{153}, \mathrm{SMI}^{152}$, and SMA-Pos ${ }^{164}$ are positively charged at neutral $\mathrm{pH}$ values. SMA-SH ${ }^{157}$ allows further functionalization with fluorescent tags, while SMA-EA-DOTA ${ }^{163}$ enables the insertion of paramagnetic ions. Finally, ST10, and ST-100 (discussed in Chapter 5) are paramagnetic SMAs, opening to EPR, PRE- and DNP-NMR studies. Table 1 summarizes a few features of each copolymer. The asterisks indicate the structures of the polymers discussed in this thesis.

For completeness, Figure 1.15 shows nanodiscs-forming non-SMA-based copolymers proposed in the literature by many research groups. 


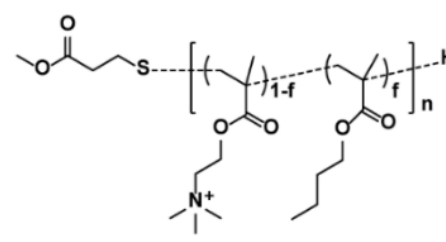

PMA

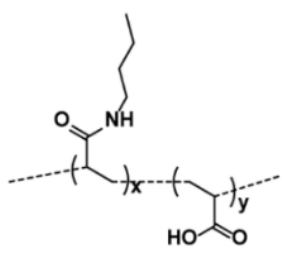

Butyl-PAA

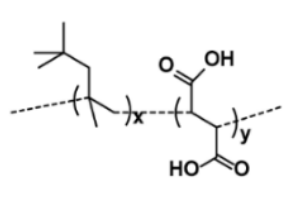

DIBMA

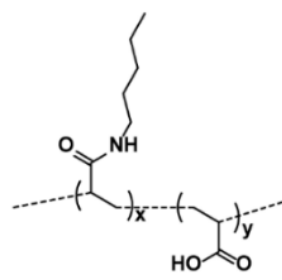

Pentyl-PAA

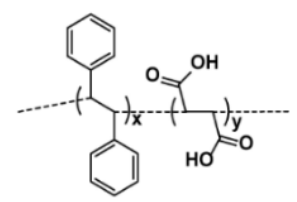

STMA

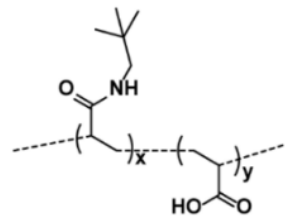

neo-pentyl-PAA

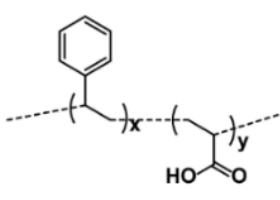

AASTY

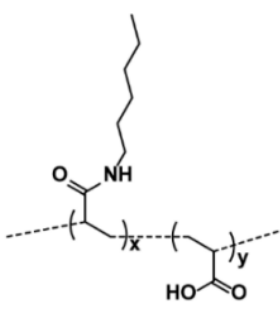

hexyl-PAA

Figure 1.15. Non-SMA copolymers for membrane protein investigations. A variety of non-SMA copolymers has been proposed. DIBMA ${ }^{150}$ is widely employed in the direct extraction and stabilization of membrane proteins. AASTY ${ }^{165}$ is derived from the copolymerization of styrene and acrylic acid monomers. STMA ${ }^{151}$ uses stilbene in place of styrene monomers, tuning the rigidity of the copolymer chains. PMAs ${ }^{158}$ (formulation N-C4-60-4.7) and PAAs ${ }^{132}$ (formulations pentyl- and hexyl-PAA) were developed in the Ramamoorthy lab and have shown to be promising in membranes solubilization. Table 1.2 summarizes a few features of each copolymer.

\begin{tabular}{|c|c|c|c|c|c|c|c|}
\hline \multirow{2}{*}{$\begin{array}{l}\text { Copolymer } \\
\text { name }\end{array}$} & \multirow{2}{*}{ Subunit ratio } & \multirow{2}{*}{$\begin{array}{l}\text { Charge } \\
\text { (at neutral pH) }\end{array}$} & \multicolumn{2}{|c|}{$\begin{array}{l}\text { Molecular weight of the } \\
\text { starting material }\end{array}$} & \multirow{2}{*}{$\begin{array}{l}\text { Reaction } \\
\text { method }\end{array}$} & \multirow[t]{2}{*}{ Year } & \multirow{2}{*}{ Reference } \\
\hline & & & $M_{w}(k D a)$ & $M_{n}(k D a)$ & & & \\
\hline DIBMA & $3: 1$ & Negative & 15.3 & 8.4 & N/A & 2017 & 150 \\
\hline PMA & $2: 1$ & Negative & $\mathrm{N} / \mathrm{A}$ & 4.7 & RAFT & 2017 & 158 \\
\hline Butyl-PAA & $1: 1$ & Negative & 1.8 & N/A & N/A & 2019 & 132 \\
\hline Pentyl-PAA & $1: 1$ & Negative & 1.8 & N/A & $\mathrm{N} / \mathrm{A}$ & 2019 & 132 \\
\hline neopentyl-PAA & $1: 1$ & Negative & 1.8 & N/A & $\mathrm{N} / \mathrm{A}$ & 2019 & 132 \\
\hline Hexyl-PAA & $1: 1$ & Negative & 1.8 & N/A & $\mathrm{N} / \mathrm{A}$ & 2019 & 132 \\
\hline STMA & $2: 1$ & Negative & N/A & $4.4-40$ & RAFT & 2020 & 151 \\
\hline AASTY & N/A* & Negative & 7.9-10.8 & $6.6-8.9$ & RAFT & 2020 & 165 \\
\hline
\end{tabular}

Table 1.2. List of non-SMA-based copolymers. A list of non-SMA-based copolymers is shown in chronological order of appearance. The table does not show RAFT variants of CSTR synthesized copolymers and does not include formulations showing different subunit (hydrophobic-to-hydrophilic) molar ratios.

Many groups synthesize their polymers. However, several vendors produce nanodiscs-forming copolymers. Currently, the commercial SMA-based products are sold as SMA® by Cray Valley (Beaufort, TX, USA), Xiran ${ }^{\circledR}$ by Polyscience (Geleen, NL), Lipodisq ${ }^{\circledR}$ by Sigma-Aldrich ${ }^{\circledR}$ (St.Louis, MO), and Scripset ${ }^{\circledR}$ by Soleniw (Wilmington, DE, USA). DIBMA is commercialized by Anatrace LLC. (Maumee, OH), and Cube Biotech® Inc. (Wayne, PA). PMA is sold by Avanti Polar Lipids Inc. (Alabaster, AL). 


\subsection{Achievements in the Ramamoorthy group}

\subsubsection{Modified SMALPs as membrane mimicking systems.}

SMA polymers have been very successful in the reconstitution of membrane proteins into nanodiscs $23,92,117,118,121,166-171$. The unique advantage over other membrane mimetics is that SMA has proven to extract membrane proteins from the cellular membrane directly ${ }^{86,92,172}$. SMA polymer nanodiscs have been shown to form in a variety of lipids with a size range of 10-15 nm diameter ${ }^{89,168}$.

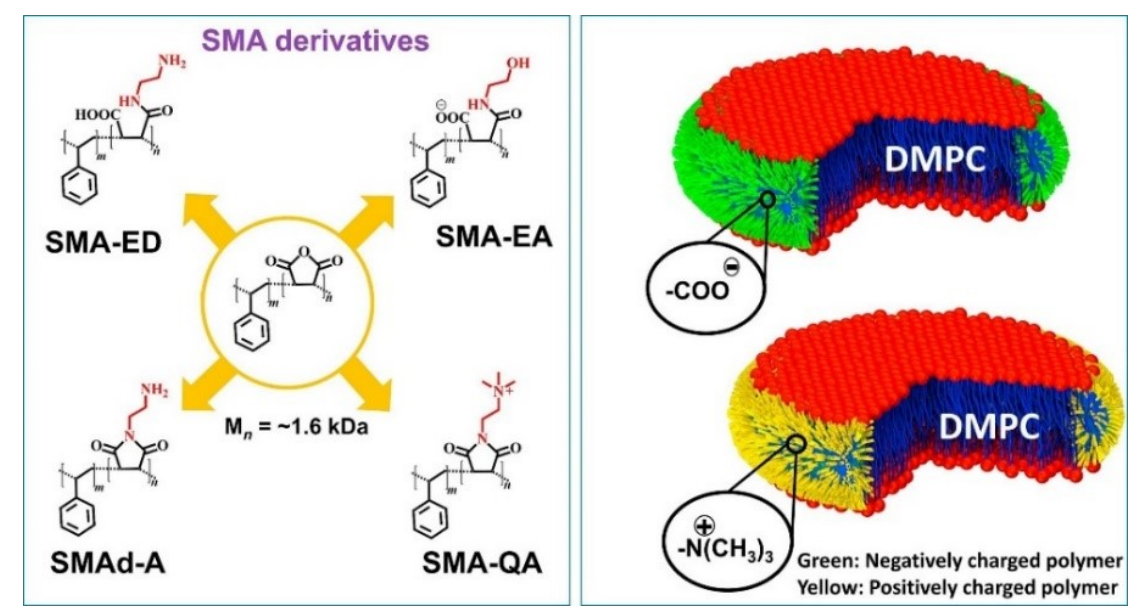

Figure 1.16. SMA derivatives that form lipid nanodiscs. Hydrophilic functionalization to enhance polymer nanodisc features and applications. Molecular structures of SMA based polymer derivatives used to form lipid-nanodiscs (left panel) and illustrations of "sushi-like" lipid-nanodiscs (right panel). As reported in our publications ${ }^{153,155,173}$, the ability of a synthetic polymer to solubilize lipid aggregates is characterized by static light scattering (SLS) experiments on multilamellar vesicles (MLVs), TIRF based fluorescence experiments, and phosphorus-31 NMR experiments. Polymerbased nanodiscs are prepared via the self-assembly process by directly mixing an appropriate ratio of polymer and lipid(s) in a buffer. Then, the mixture is incubated and purified by size-exclusion chromatography (SEC). The purified nanodiscs are characterized by dynamic light scattering (DLS) experiments to determine the size distribution and transmission electron microscopy (TEM) images to evaluate the size homogeneity of polymer-based lipid-nanodiscs. Finally, the isotropic nanodiscs are further characterized by solution NMR spectroscopy, while the anisotropic macro-nanodiscs are analyzed using a variety of solid-state NMR experiments, including ${ }^{31} \mathrm{P},{ }^{14} \mathrm{~N}, 2 \mathrm{D}{ }^{1} \mathrm{H} /{ }^{1} \mathrm{H}$ RFDR, SLF, and $2 \mathrm{D}{ }^{1} \mathrm{H} /{ }^{13} \mathrm{C}$ HETCOR. Based on the experience gained and different protocols used in the investigation of a variety of polymers, lipids, and proteins in the Ramamoorthy lab, the use of freeze-thaw cycles for some of the lipids, including POPC, POPG, and DSPC, is required in the preparation of polymer-based nanodiscs; further optimization may be required depending on the sample/system under investigation. In addition, sample preparation procedures for a successful reconstitution of a membrane protein need to be adapted based on the physicochemical properties of the protein under investigation. For a given membrane protein, magnetic alignment of macro-nanodiscs (large-size nanodiscs) and successful implementation of solid-state NMR experiments require optimization of various parameters, including the lipid:polymer ratio, concentration of nanodiscs, amount of paramagnetic (for example, lanthanide) salt, and temperature. Copyright 2018 Elsevier. 
A variety of biophysical studies reported the characterization of SMA based polymer nanodiscs ${ }^{128,174,175}$. NMR experiments have been used to show the formation of nanodiscs and to probe lipid dynamics ${ }^{176,177}$. Due to the lack of relative size control, the current NMR applications of polymer nanodiscs are limited to solution NMR. In order to expand the NMR applications of polymer nanodiscs to both solution and solid-state NMR, more control over nanodiscs size is needed ${ }^{39,69,178}$. Our hypothesis is that a lower molecular weight polymer would allow for size control of nanodiscs is based on the knowledge gained from the molecular weight (MW) difference between high MW MSPs, which do not qualify for size control, and low MW peptide-based nanodiscs, which have been demonstrated to enable size control ${ }^{52,72}$. Our lab has been focused on using low molecular weight polymers to achieve the necessary nanodiscs size control for various biophysical and structural biology studies.

\subsubsection{Expanding the range of sizes nanodiscs by using SMA-EA}

The first functionalized SMA based polymer developed in the Ramamoorthy lab was styrene-maleic anhydride-ethanolamine (SMA-EA) ${ }^{173}$. The used starting material was a commercially available poly(styrene-co-maleic anhydride) cumene terminated with $\sim 1.3: 1$ styrene:maleic anhydride molar ratio with a number average molecular weight of about $1.6 \mathrm{kDa}$. SMA-EA was synthesized by modifying the starting SMAnh via a nucleophilic ring-opening reaction using ethanolamine. The resulting polymer was shown to form nanodiscs with large sizes (10-60 nm diameter) by varying the lipid to polymer ratio. Small SMA-EA nanodiscs, with lipid:polymer $(\mathrm{w} / \mathrm{w}) \sim 2,(<20 \mathrm{~nm})$ were shown to be suitable for solution NMR experiments, whereas the largest-sized nanodiscs (macro-nanodiscs) (lipid:polymer 1:1 w/w) aligned in the presence of an external magnetic field enabling structural studies of membrane proteins using solid-state NMR spectroscopy ${ }^{179-181}$. The ability of nanodiscs to be used in the study of membrane proteins using solution as well as solid-state NMR were shown using Cyt b5 as an example. Uniformly- ${ }^{15} \mathrm{~N}$-labeled Cytochrome-b5 reconstituted in small nanodiscs $(10 \mathrm{~nm})$ exhibited well-dispersed peaks in a 2D TROSY-HSQC (transverse relaxation optimized spectroscopy-heteronuclear single quantum correlation) spectrum suggesting that protein is well folded. Cyt b5 reconstituted in macro-nanodiscs were used in a 2D PISEMA (polarization inversion and spin-exchange at the magic angle) experiment ${ }^{180,182-184}$. The PISEMA spectrum revealed a characteristic wheel-like pattern of resonances showing the tilt of the helical transmembrane domain with respect to the lipid bilayer normal. The SMA-EA polymer was also shown to have increased stability towards divalent metal ions (up to $21 \mathrm{mM}$ for $\mathrm{Ca}^{2+}$ and $30 \mathrm{mM}$ for $\mathrm{Mg}^{2+}$ ), and increased tolerance towards low $\mathrm{pH}$ (from 4.5 to $\mathrm{pH} 3.3$ ) based on the lipid:polymer w/w ratio)) as compared to SMALP $(\mathrm{pH} \sim 6.3$ ). While we demonstrated that SMA-EA polymer nanodiscs could be formed in 
a wide variety of sizes, the presence of carboxylic groups as the hydrophilic component still limited the application of SMA-EA nanodiscs.

\subsubsection{Acidic compatible polymer nanodiscs using SMA-ED and} SMAd-A

Because amines readily react with the SMAnh anhydride group, we hypothesized that substituting the carboxylic acids with other hydrophilic groups would increase the polymer nanodiscs' stability towards low $\mathrm{pH}$. To test this hypothesis, we synthesized two different SMA derivatives, styrenemaleic acid-ethylene diamine (SMA-ED) and styrene maleimide-amine (SMAd-A) ${ }^{155}$. First, we synthesized SMA-ED, a zwitterionic analog of SMA, with the idea that the polymer would always be charged due to the negatively charged carboxylates at high $\mathrm{pH}$ and the positively charged amino groups at low $\mathrm{pH}$. This polymer showed stability for a wide range of $\mathrm{pH}$ values $(3.5<\mathrm{pH}<8.5)$ during the presence of only one kind of charged functional group. One interesting result was that SMA-ED was not stable and precipitated from solution at the near-neutral environment ( $\mathrm{pH} \sim 6+/$ 1). We interpreted this observation as being a result of the zwitterionic state and SMA-ED forming aggregates with itself due to the presence of attractive charge-charge interactions. SMAd-A was synthesized to show a monofunctional form of SMA that is stable at low $\mathrm{pH}$. The SMAd-A polymer was formed from a Boc-protected SMA-ED dehydration reaction to form the maleimide, followed by a Boc deprotection. This positively charged SMAd-A demonstrated the expected characteristic stability at acidic conditions $(\mathrm{pH}=3.5)$ while it precipitated at basic conditions $(\mathrm{pH}>6)$ due to the loss of charge on the amino functional groups. SMA-ED and SMAd-A both showed remarkable stability towards the presence of divalent metal ions at acidic $\mathrm{pH}$. These results demonstrated that the replacement of the metal-chelating carboxylates with non-chelating amino groups allows for the formation of nanodiscs even in the presence of divalent metal ions and at lower $\mathrm{pH}$. Both SMA-ED and SMAd-A based lipid nanodiscs have also been demonstrated to stabilize a medically important polyphenolic compound, curcumin, showing a potential application in the field of drug delivery ${ }^{155}$.

\subsubsection{Robust $\mathrm{pH}$ resistant nanodiscs using SMA-QA}

Both SMA-ED and SMAd-A still had limitations due to pH-dependent hydrophilic groups, which restricted their use to specific $\mathrm{pH}$ ranges. With the goal to form polymer nanodiscs that are stable under all biologically relevant $\mathrm{pH}$ and metal ion concentrations, we synthesized styrene maleimidequaternary ammonium (SMA-QA) polymer ${ }^{153}$. We selected quaternary ammonium as the charged hydrophilic group due to its $\mathrm{pH}$-independent charge and non-chelating properties. SMA-QA was synthesized using a similar approach previously seen in our SMAd-A polymer synthesis. The 
functionalization was performed by reacting SMA $(\sim 1.6 \mathrm{kDa})$ with aminoethyltrimethylammonium chloride hydrochloride followed by a dehydration reaction forming the maleimide. SMA-QA was shown to form monodispersed nanodiscs of various sizes $(10-30 \mathrm{~nm})$ by varying the lipid to polymer ratio $(1: 1.5$ to $1: 0.25 \mathrm{w} / \mathrm{w})$. These nanodiscs were shown to be monodispersed in size as characterized by dynamic light scattering (DLS), transmission electron microscopy (TEM), and size exclusion chromatography (SEC). The smaller nanodiscs (about $10 \mathrm{~nm}$ ) were shown to be isotropic in solution, enabling solution NMR studies on membrane proteins, whereas the macro-nanodiscs $(>20 \mathrm{~nm})$ exhibited spontaneous alignment in the magnetic field and are suitable for solid-state NMR studies. The remarkable feature of SMA-QA nanodiscs is that the nanodiscs are stable in a wide range of $\mathrm{pH}$ values $(2.5<\mathrm{pH}<10)$ and in the presence of divalent metal ions (up to $200 \mathrm{mM}$ of $\mathrm{Ca}^{2+}$ or $\mathrm{Mg}^{2+}$ ). SMA-QA nanodiscs are a robust membrane mimetic tool that offers significant advantages over all currently reported nanodisc systems because of these unique properties. Therefore, we foresee a substantial expansion in the applicability of nanodisc technology.

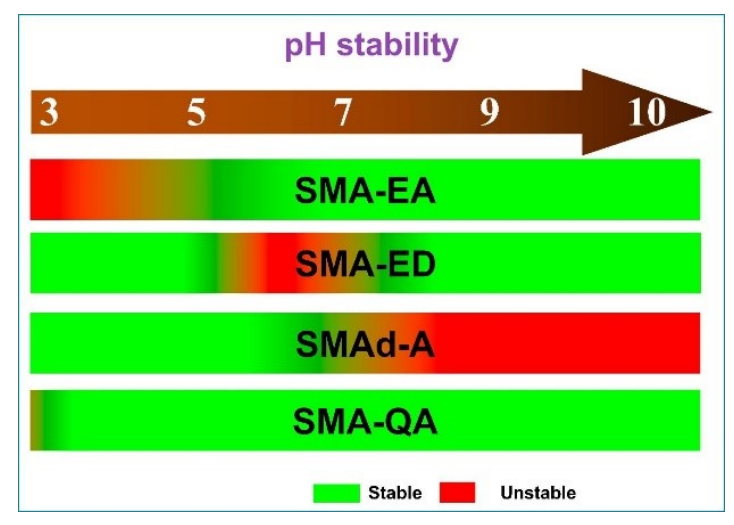

Figure 1.17. pH stability of polymer nanodiscs. A schematic representation of the stability of various polymer nanodiscs under different $\mathrm{pH}$ values based on the experimental characterization as reported elsewhere ${ }^{153,155,185}$. Copyright 2018 Elsevier.

Recent studies have demonstrated that many advantages of SMA based polymer over peptidic based nanodiscs, which includes studies on membrane proteins ${ }^{107,108,171,186-191}$. However, the functional modifications described here would drastically increase the applications of these polymer-based nanodiscs in studying membrane proteins using a wide variety of biophysical techniques including solution and solid-state NMR spectroscopy. Most of the polymer nanodiscs studies on membrane proteins reported in the literature are limited to neutral $\mathrm{pH}$. In contrast, SMA-QA polymer can be used to study the $\mathrm{pH}$-dependent membrane insertion or function of a membrane protein even at low $\mathrm{pH}^{160,190}$. We also would like to mention that a strong UV absorption and the interactions with the aromatic styrene group of SMA-based polymers pose limitations for some biophysical techniques. 
For example, the SMA polymer has been shown to have strong interactions with Thioflavin T (ThT) dye that is commonly used in monitoring the kinetics of amyloid aggregation. To overcome these limitations of polymer-based nanodiscs, recent developments have focused on producing styrene-free polymers, which include diisobutylene/maleic acid copolymer ${ }^{169}$ and poly-methacrylate copolymers $^{191}$.

\subsection{Conclusions and impacts}

Membrane mimicking systems are crucial for the investigation of membrane proteins, and among them, SMAs and SMALPs proved to be invaluable tools. In conclusion, functionalization of a low molecular weight SMA polymer allowed for the tuning and enhancement of these polymers to different $\mathrm{pH}$ and metal ion presence while also allowing greater control over size. Using size control, polymer nanodiscs' applications in the field of NMR spectroscopy, specifically for structural studies using solid-state NMR spectroscopy, is achievable. We expect that macro-nanodiscs will prove to be a valuable tool in studying functional reconstitution and structural investigation of large-size membrane proteins and membrane protein assemblies like channels and complexes. These macronanodiscs will allow the use of sophisticated biophysical techniques, including cryo-EM, SAXS, and SANS. Overall, using a straightforward chemical functionalization to modify the functional groups of SMA polymers enables us to engineer new polymers to overcome the current limitations seen in polymer nanodiscs technology. We have also demonstrated the use of styrene-free polymers to form lipid-nanodiscs, which have been potentially useful in investigating amyloid aggregation and for studies using styrene-sensitive biophysical experiments such as CD and ThT based fluorescence ${ }^{191}$. This allows us to greatly expand the use of polymer nanodiscs for biophysical structural and functional studies on a variety of membrane proteins, membrane-bound protein-protein complexes, and domains of cell membranes. It is also worthwhile to explore the feasibility of potential biomedical applications of polymer-based nanodiscs.

The introduction of nanodisc-forming polymers circumvented the need for the initial detergent extraction, but currently available copolymers still have several shortcomings. We believe that further optimization of the polymers would be useful to continue to expand the applications of polymer nanodiscs. Some of the polymer aspects worth investigating include the following. First, the optimization of the styrene and maleic acid group alternation in the chain (perfect alternating versus statistically random) may provide additional insights into protein structural biology using nanodiscs. Second, the design of a variety of hydrophobic groups to accommodate the variation in the 
hydrophobic thickness of the lipid bilayer while eliminating the styrene moiety due to its potential interaction with proteins. Third, it is also essential to further investigate the lamellar nature of lipids in SMA based nanodiscs using calorimetry and solid-state NMR based experiments and to systematically investigate the effect of the hydrophobic groups of the polymers on the structure and function of the reconstituted protein(s) and the physicochemical properties of lipids. Finally, further studies to optimize the reconstitution of a variety of membrane composition, asymmetric lipid bilayer, and raft-like domains would further broaden the scope of polymer-based nanodiscs. 


\section{References}

(1) Singer, S. J.; Nicolson, G. L. The Fluid Mosaic Model of the Structure of Cell Membranes. Science (80). 1972, 175 (4023), 720-731.

(2) Engelman, D. M. Membranes Are More Mosaic than Fluid. Nature 2005, 438, 8-10.

(3) Van Meer, G.; De Kroon, A. I. P. M. Lipid Map of the Mammalian Cell. J. Cell Sci. 2011, 124 (1), 5-8.

(4) Nagle, J. F.; Tristram-Nagle, S. Structure of Lipid Bilayers. Biochim. Biophys. Acta - Rev. Biomembr. 2000, 1469 (3), 159-195.

(5) Tristram-Nagle, S.; Nagle, J. F. Lipid Bilayers: Thermodynamics, Structure, Fluctuations, and Interactions. Chem. Phys. Lipids 2004, 127, 3-14.

(6) El-Bacha, T.; Torres, A. G. Phospholipids: Physiology, 1st ed.; Elsevier Ltd., 2015.

(7) Harayama, T.; Riezman, H. Understanding the Diversity of Membrane Lipid Composition. 2018.

(8) Ye, L.; Neale, C.; Sljoka, A.; Lyda, B.; Pichugin, D.; Tsuchimura, N.; Larda, S. T.; Pomès, R.; García, A. E.; Ernst, O. P.; et al. Mechanistic Insights into Allosteric Regulation of the A2Aadenosine G Protein-Coupled Receptor by Physiological Cations. Nat. Commun. 2018, 9 (1), 1-13.

(9) Prosser, R. S.; Ye, L.; Pandey, A.; Orazietti, A. Activation Processes in Ligand-Activated G Protein-Coupled Receptors: A Case Study of the Adenosine A2Areceptor. BioEssays 2017, 39 (9), 1-10.

(10) Ye, L.; Van Eps, N.; Zimmer, M.; Ernst, O. P.; Scott Prosser, R. Activation of the A2A Adenosine G-Protein-Coupled Receptor by Conformational Selection. Nature 2016, 533 (7602), 265-268. 
(11) Cournia, Z.; Allen, T. W.; Andricioaei, I.; Antonny, B.; Baum, D.; Brannigan, G.; Buchete, N.-V.; Deckman, J. T.; Delemotte, L.; del Val, C.; et al. Membrane Protein Structure, Function, and Dynamics: A Perspective from Experiments and Theory. J. Membr. Biol. 2015, 248, 611640.

(12) Fry, M. Y.; Clemons, W. M. J. Complexity in Targeting Membrane Proteins. Science (80-. ). 2018, 359 (6374), 390-391.

(13) Nicolson, G. L. The Fluid - Mosaic Model of Membrane Structure: Still Relevant to Understanding the Structure, Function and Dynamics of Biological Membranes after More than 40 Years. Biochim. Biophys. Acta - Biomembr. 2014, 1838 (6), 1451-1466.

(14) Wikimedia

Commons

source: https://commons.wikimedia.org/wiki/File:0303_Lipid_Bilayer_With_Various_Components.j $\mathrm{pg}$.

(15) Wallin, E.; Heijne, G. Von. Genome-Wide Analysis of Integral Membrane Proteins from Eubacterial, Archaean, and Eukaryotic Organisms. Protein Sci. 1998, 7 (4), 1029-1038.

(16) Overington, J. P.; Al-Lazikani, B.; Hopkins, A. L. How Many Drug Targets Are There? Nat. Rev. Drug Discov. 2006, 5 (12), 993-996.

(17) Drews, J. Drug Discovery: A Historical Perspective. Science (80). 2000, 287 (5460), 1960 LP $-1964$.

(18) Stenson, P. D.; Mort, M.; Ball, E. V.; Shaw, K.; Phillips, A. D.; Cooper, D. N. The Human Gene Mutation Database: Building a Comprehensive Mutation Repository for Clinical and Molecular Genetics, Diagnostic Testing and Personalized Genomic Medicine. Hum. Genet. 2014, $133(1), 1-9$.

(19) Rawson, S.; Davies, S.; Lippiat, J. D.; Muench, S. P. The Changing Landscape of Membrane Protein Structural Biology through Developments in Electron Microscopy. Molecular Membrane Biology. 2016, 12-22.

(20) Parmar, M. J.; De, C.; Lousa, M.; Muench, S. P.; Goldman, A.; Postis, V. L. G. Artificial Membranes for Membrane Protein Purification, Functionality and Structure Studies. Biochem. Soc. Trans. 2016, 44, 877-882.

(21) Schreiber, S. L. Target-Oriented and Diversity-Oriented Organic Synthesis in Drug Discovery. Science. Springer-VerlagMcGraw-HillWileySpringer-VerlagWileyRonald PressElsevier 
Chicago PressMcGraw-Hill 2000, 1964-1969.

(22) Frey, L.; Lakomek, N. A.; Riek, R.; Bibow, S. Micelles, Bicelles, and Nanodiscs: Comparing the Impact of Membrane Mimetics on Membrane Protein Backbone Dynamics. Angew. Chemie - Int. Ed. 2017, 56, 380-383.

(23) Hu, Z.; Ho, J. C. S.; Nallani, M. Synthetic (Polymer) Biology (Membrane): Functionalization of Polymer Scaffolds for Membrane Proteins. Curr. Opin. Biotechnol. 2017, 46, 51-56.

(24) Anandan, A.; Vrielink, A. Detergents in Membrane Protein Purification and Crystallisation. In The Next Generation in Membrane Protein Structure Determination; Moraes, I., Ed.; Springer International Publishing: Cham, 2016; 13-28.

(25) Chipot, C.; Dehez, F.; Schnell, J. R.; Zitzmann, N.; Pebay-Peyroula, E.; Catoire, L. J.; Miroux, B.; Kunji, E. R. S.; Veglia, G.; Cross, T. A.; et al. Perturbations of Native Membrane Protein Structure in Alkyl Phosphocholine Detergents: A Critical Assessment of NMR and Biophysical Studies. Chem. Rev. 2018, 118 (7), 3559-3607.

(26) Arnold, T.; Linke, D. The Use of Detergents to Purify Membrane Proteins. Curr. Protoc. Protein Sci. 2008, Chapter 4, 4.8.1-4.8.30.

(27) Garavito, R. M.; Ferguson-Miller, S. Detergents as Tools in Membrane Biochemistry. J. Biol. Chem. 2001, 276 (35), 32403-32406.

(28) Overduin, M.; Esmaili, M. Memtein: The Fundamental Unit of Membrane-Protein Structure and Function. Chem. Phys. Lipids 2019, 218, 73-84.

(29) Marsh, D. Protein Modulation of Lipids, and Vice-Versa, in Membranes. 2008.

(30) Yeagle, P. L. Non-Covalent Binding of Membrane Lipids to Membrane Proteins. Biochim. Biophys. Acta - Biomembr. 2014, 1838 (6), 1548-1559.

(31) Helenius, A. R. I.; Simons, K. A. I. Solubilization of Membrandes by Detergents. Biochim. Biophys. Acta 1975, 415, 29-79.

(32) Tanford, C.; Reynolds, J. A. Characterization of Membrane Proteins in Detergent Solutions. Biochim. Biophys. Acta - Rev. Biomembr. 1976, 457 (2), 133-170.

(33) Bordier, C. Phase Separation of Integral Membrane Proteins in Triton X-114 Solution. J. Biol. Chem. 1981, 256 (4), 1604-1607.

(34) Seddon, A. M.; Curnow, P.; Booth, P. J. Membrane Proteins, Lipids and Detergents: Not Just 
a Soap Opera. 2004.

(35) Linke, D. Chapter 34 Detergents. An Overview. Methods in Enzymology. 0076-6879th ed. Elsevier 2009, 603-617.

(36) Rigaud, J.-L.; Lévy, D. Reconstitution of Membrane Proteins into Liposomes. Methods Enzym. 2003, 372, 65-86.

(37) Rigaud, J. L.; Paternostre, M. T.; Bluzat, A. Mechanisms of Membrane Protein Insertion into Liposomes during Reconstitution Procedures Involving the Use of Detergents. 2. Incorporation of the Light-Driven Proton Pump Bacteriorhodopsin. Biochemistry 1988, 27 (8), 2677-2688.

(38) Amati, A. M.; Graf, S.; Deutschmann, S.; Dolder, N.; von Ballmoos, C. Current Problems and Future Avenues in Proteoliposome Research. Biochem. Soc. Trans. 2020, 48 (4), 1473-1492.

(39) Dürr, U. H. N.; Gildenberg, M.; Ramamoorthy, A. The Magic of Bicelles Lights up Membrane Protein Structure. Chem. Rev. 2012, 112 (11), 6054-6074.

(40) Sanders, C. R.; Prosser, R. S. Bicelles: A Model Membrane System for All Seasons? Structure 1998, $6(10), 1227-1234$.

(41) Sanders, C. R.; Landis, G. C. Reconstitution of Membrane Proteins into Lipid-Rich Bilayered Mixed Micelles for NMR Studies. Biochemistry 1995, 34 (12), 4030-4040.

(42) Soong, R.; Xu, J.; Ramamoorthy, A. Bicelles - A Much Needed Magic Wand to Study Membrane Proteins by NMR Spectroscopy. In Nuclear Magnetic Resonance Spectroscopy of Liquid Crystals; WORLD SCIENTIFIC, 2009; 117-128.

(43) Beaugrand, M.; Arnold, A. A.; Hénin, J.; Warschawski, D. E.; Williamson, P. T. F.; Marcotte, I. Lipid Concentration and Molar Ratio Boundaries for the Use of Isotropic Bicelles. Langmuir 2014, 30 (21), 6162-6170.

(44) Sanders, C. R.; Schwonek, J. P. Characterization of Magnetically Orientable Bilayers in Mixtures of Dihexanoylphosphatidylcholine and Dimyristoylphosphatidylcholine by SolidState NMR. Biochemistry 1992, 31 (37), 8898-8905.

(45) Sanders, C. R.; Hare, B. J.; Howard, K. P.; Prestegard, J. H. Magnetically-Oriented Phospholipid Micelles as a Tool for the Study of Membrane-Associated Molecules. Progress in Nuclear Magnetic Resonance Spectroscopy. Pergamon, 1994, 421-444.

(46) Cardon, T. B.; Dave, P. C.; Lorigan, G. A. Magnetically Aligned Phospholipid Bilayers with 
Large q Ratios Stabilize Magnetic Alignment with High Order in the Gel and La Phases. Langmuir 2005, 21 (10), 4291-4298.

(47) Yamamoto, K.; Soong, R.; Ramamoorthy, A. Comprehensive Analysis of Lipid Dynamics Variation with Lipid Composition and Hydration of Bicelles Using Nuclear Magnetic Resonance (NMR) Spectroscopy. Langmuir 2009, 25 (12), 7010-7018.

(48) Denisov, I. G.; Sligar, S. G. Nanodiscs for Structural and Functional Studies of Membrane Proteins. Nat. Struct. Mol. Biol. 2016, 23 (6), 481-486.

(49) Dürr, U. H. N.; Soong, R.; Ramamoorthy, A. When Detergent Meets Bilayer: Birth and Coming of Age of Lipid Bicelles. Prog. Nucl. Magn. Reson. Spectrosc. 2013, 69, 1-22.

(50) Sanders, C. R.; Landis, G. C. Reconstitution of Membrane-Proteins Into Lipid-Rich Bilayered Mixed Micelles for Nmr-Studies. Biochemistry 1995, 34, 4030-4040.

(51) Etzkorn, M.; Viegas, A.; Viennet, T.; Viegas, R. A. The Power, Pitfalls and Potential of the Nanodisc System for NMR-Based Studies. Biol. Chem 2016, 397 (12), 1335-1354.

(52) Denisov, I. G.; Sligar, S. G. Nanodiscs in Membrane Biochemistry and Biophysics. Chemical Reviews. 2017, 4669-4713.

(53) Hardy, G. J.; Nayak, R.; Zauscher, S. Model Cell Membranes: Techniques to Form Complex Biomimetic Supported Lipid Bilayers via Vesicle Fusion. Curr. Opin. Colloid Interface Sci. 2013, $18(5), 448-458$.

(54) Glazier, R.; Salaita, K. Supported Lipid Bilayer Platforms to Probe Cell Mechanobiology. Biochim. Biophys. Acta - Biomembr. 2017, 1859 (9), 1465-1482.

(55) Zoonens, M.; Popot, J. L. Amphipols for Each Season. J. Membr. Biol. 2014, 247 (9-10), 759796.

(56) Tribet, C.; Audebert, R.; Popot, J. L. Amphipols: Polymers That Keep Membrane Proteins Soluble in Aqueous Solutions. Proc. Natl. Acad. Sci. U. S. A. 1996, 93 (26), 15047-15050.

(57) Liao, M.; Cao, E.; Julius, D.; Cheng, Y. Structure of the TRPV1 Ion Channel Determined by Electron Cryo-Microscopy. Nature 2013, 504 (7478), 107-112.

(58) Bai, X.; Yan, C.; Yang, G.; Lu, P.; Ma, D.; Sun, L.; Zhou, R.; Scheres, S. H. W.; Shi, Y. An Atomic Structure of Human $\gamma$-Secretase. Nature 2015, 525 (7568), 212-217.

(59) Mazhab-Jafari, M. T.; Rohou, A.; Schmidt, C.; Bueler, S. A.; Benlekbir, S.; Robinson, C. V.; 
Rubinstein, J. L. Atomic Model for the Membrane-Embedded VO Motor of a Eukaryotic VATPase. Nature 2016, 539 (7627), 118-122.

(60) Wilkes, M.; Madej, M. G.; Kreuter, L.; Rhinow, D.; Heinz, V.; De Sanctis, S.; Ruppel, S.; Richter, R. M.; Joos, F.; Grieben, M.; et al. Molecular Insights into Lipid-Assisted Ca 2+ Regulation of the TRP Channel Polycystin-2. Nat. Struct. Mol. Biol. 2017, 24 (2), 123-130.

(61) Popot, J.-L.; Althoff, T.; Bagnard, D.; Banères, J.-L.; Bazzacco, P.; Billon-Denis, E.; Catoire, L. J.; Champeil, P.; Charvolin, D.; Cocco, M. J.; et al. Amphipols From A to Z*. Annu. Rev. Biophys. 2011, 40 (1), 379-408.

(62) Bayburt, T. H.; Grinkova, Y. V; Sligar, S. G. Self-Assembly of Discoidal Phospholipid Bilayer Nanoparticles with Membrane Scaffold Proteins. Nano Lett. 2002, 2 (8), 853-856.

(63) Nath, A.; Atkins, W. M.; Sligar, S. G. Applications of Phospholipid Bilayer Nanodiscs in the Study of Membranes and Membrane Proteins. Biochemistry. 2007, 2059-2069.

(64) Denisov, I. G.; Sligar, S. G. Nanodiscs for Structural and Functional Studies of Membrane Proteins. Nat. Struct. Mol. Biol. 2016, 23 (6), 481-486.

(65) Bayburt, T. H.; Carlson, J. W.; Sligar, S. G. Reconstitution and Imaging of a Membrane Protein in a Nanometer-Size Phospholipid Bilayer. J. Struct. Biol. 1998, 123 (1), 37-44.

(66) Hagn, F.; Etzkorn, M.; Raschle, T.; Wagner, G. Optimized Phospholipid Bilayer Nanodiscs Facilitate High-Resolution Structure Determination of Membrane Proteins. J. Am. Chem. Soc. 2013, 135 (5), 1919-1925.

(67) Nasr, M. L.; Baptista, D.; Strauss, M.; Sun, Z.-Y. J.; Grigoriu, S.; Huser, S.; Plückthun, A.; Hagn, F.; Walz, T.; Hogle, J. M.; et al. Covalently Circularized Nanodiscs for Studying Membrane Proteins and Viral Entry. Nat. Methods 2017, 14 (1), 49-52.

(68) Ritchie, T. K.; Grinkova, Y. V.; Bayburt, T. H.; Denisov, I. G.; Zolnerciks, J. K.; Atkins, W. M.; Sligar, S. G. Reconstitution of Membrane Proteins in Phospholipid Bilayer Nanodiscs; 2009; 211-231.

(69) Hagn, F.; Nasr, M. L.; Wagner, G. Assembly of Phospholipid Nanodiscs of Controlled Size for Structural Studies of Membrane Proteins by NMR. Nat. Publ. Gr. 2017, 13.

(70) Kay, L. E.; Rodriguez Camargo, D. C.; Korshavn, K. J.; Jussupow, A.; Raltchev, K.; Goricanec, D.; Fleisch, M.; Sarkar, R.; Xue, K.; Aichler, M.; et al. Stabilization and Structural Analysis of a Membrane-Associated HIAPP Aggregation Intermediate. 2017. 
(71) Nath, A.; Miranker, A. D.; Rhoades, E. A Membrane-Bound Antiparallel Dimer of Rat Islet Amyloid Polypeptide. Angew. Chemie Int. Ed. 2011, 50 (46), 10859-10862.

(72) Zhang, M.; Huang, R.; Ackermann, R.; Im, S. C.; Waskell, L.; Schwendeman, A.; Ramamoorthy, A. Reconstitution of the Cytb5-CytP450 Complex in Nanodiscs for Structural Studies Using NMR Spectroscopy. Angew. Chemie - Int. Ed. 2016, 55 (14), 4497-4499.

(73) Ravula, T.; Barnaba, C.; Mahajan, M.; Anantharamaiah, G. M.; Im, S. C.; Waskell, L.; Ramamoorthy, A. Membrane Environment Drives Cytochrome P450’s Spin Transition and Its Interaction with Cytochrome: B5. Chem. Commun. 2017, 53 (95), 12798-12801.

(74) Ravula, T.; Ishikuro, D.; Kodera, N.; Ando, T.; Anantharamaiah, G. M.; Ramamoorthy, A. Real-Time Monitoring of Lipid Exchange via Fusion of Peptide Based Lipid-Nanodiscs. Chem. Mater. 2018, 30 (10), 3204-3207.

(75) Barnaba, C.; Sahoo, B. R.; Ravula, T.; Medina-Meza, I. G.; Im, S.-C.; Anantharamaiah, G. M.; Waskell, L.; Ramamoorthy, A. Cytochrome-P450-Induced Ordering of Microsomal Membranes Modulates Affinity for Drugs. Angew. Chemie 2018, 130 (13), 3449-3453.

(76) Barnaba, C.; Ravula, T.; Medina-Meza, I. G.; Im, S.-C.; Anantharamaiah, G. M.; Waskell, L.; Ramamoorthy, A. Lipid-Exchange in Nanodiscs Discloses Membrane Boundaries of Cytochrome-P450 Reductase. 2018, 1-3.

(77) Prade, E.; Mahajan, M.; Im, S.; Zhang, M.; Gentry, K. A.; Anantharamaiah, G. M.; Waskell, L.; Ramamoorthy, A. A Minimal Functional Complex of Cytochrome P450 and FBD of Cytochrome P450 Reductase in Nanodiscs. Angew. Chemie Int. Ed. 2018, 57, 1-6.

(78) Park, S. H.; Berkamp, S.; Cook, G. A.; Chan, M. K.; Viadiu, H.; Opella, S. J. Nanodiscs versus Macrodiscs for NMR of Membrane Proteins. Biochemistry 2011, 50 (42), 8983-8985.

(79) Kuai, R.; Ochyl, L. J.; Bahjat, K. S.; Schwendeman, A.; Moon, J. J. Designer Vaccine Nanodiscs for Personalized Cancer Immunotherapy. Nat. Mater. 2016, 16, 489.

(80) Kuai, R.; Li, D.; Chen, Y. E.; Moon, J. J.; Schwendeman, A. High-Density Lipoproteins: Nature's Multifunctional Nanoparticles. ACS Nano 2016, 10 (3), 3015-3041.

(81) Popovic, K.; Holyoake, J.; Pomès, R.; Privé, G. G. Structure of Saposin A Lipoprotein Discs. Proc. Natl. Acad. Sci. U. S. A. 2012, 109 (8), 2908-2912.

(82) Frauenfeld, J.; Löving, R.; Armache, J. P.; Sonnen, A. F.-P.; Guettou, F.; Moberg, P.; Zhu, L.; Jegerschöld, C.; Flayhan, A.; Briggs, J. A. G.; et al. A Saposin-Lipoprotein Nanoparticle 
System for Membrane Proteins. Nat. Methods 2016, 13 (4), 345-351.

(83) Carlson, M. L.; Young, J. W.; Zhao, Z.; Fabre, L.; Jun, D.; Li, J.; Li, J.; Dhupar, H. S.; Wason, I.; Mills, A. T.; et al. The Peptidisc, a Simple Method for Stabilizing Membrane Proteins in Detergent-Free Solution. Elife 2018, 7, 1-23.

(84) Neumann, B.; Chao, K.; Chang, C. C. Y.; Chang, T. Y. Nanodisc Scaffold Peptide (NSPr) Replaces Detergent by Reconstituting Acyl-CoA:Cholesterol Acyltransferase 1 into Peptidiscs. Arch. Biochem. Biophys. 2020, 691, 108518.

(85) Angiulli, G.; Dhupar, H. S.; Suzuki, H.; Wason, I. S.; Duong Van Hoa, F.; Walz, T. New Approach for Membrane Protein Reconstitution into Peptidiscs and Basis for Their Adaptability to Different Proteins. Elife 2020, 9, 1-20.

(86) Dörr, J. M.; Koorengevel, M. C.; Schäfer, M.; Prokofyev, A. V; Scheidelaar, S.; Van Der Cruijsen, E. A. W.; Dafforn, T. R.; Baldus, M.; Killian, J. A. Detergent-Free Isolation, Characterization, and Functional Reconstitution of a Tetrameric $\mathrm{K}^{+}$Channel: The Power of Native Nanodiscs. Proc. Natl. Acad. Sci. U. S. A. 2014, 111 (52), 18607-18612.

(87) Lee, S. C.; Knowles, T. J.; Postis, V. L. G.; Jamshad, M.; Parslow, R. A.; Lin, Y.; Goldman, A.; Sridhar, P.; Overduin, M.; Muench, S. P.; et al. A Method for Detergent-Free Isolation of Membrane Proteins in Their Local Lipid Environment. Nat. Protoc. 2016, 11 (7), 1149-1162.

(88) Scheidelaar, S.; Koorengevel, M. C.; van Walree, C. A.; Dominguez, J. J.; Dörr, J. M.; Killian, J. A. Effect of Polymer Composition and PH on Membrane Solubilization by Styrene-Maleic Acid Copolymers. Biophys. J. 2016, 111 (9), 1974-1986.

(89) Orwick, M. C.; Judge, P. J.; Procek, J.; Lindholm, L.; Graziadei, A.; Engel, A.; Gröbner, G.; Watts, A. Detergent-Free Formation and Physicochemical Characterization of Nanosized Lipid-Polymer Complexes: Lipodisq. Angew. Chemie - Int. Ed. 2012.

(90) Tonge, S. R.; Tighe, B. J. Responsive Hydrophobically Associating Polymers: A Review of Structure and Properties. Advanced Drug Delivery Reviews. 2001, 109-122.

(91) Knowles, T. J.; Finka, R.; Smith, C.; Lin, Y.-P.; Dafforn, T.; Overduin, M. Membrane Proteins Solubilized Intact in Lipid Containing Nanoparticles Bounded by Styrene Maleic Acid Copolymer. J. Am. Chem. Soc. 2009, 131 (22), 7484-7485.

(92) Lee, S. C.; Knowles, T. J.; Postis, V. L. G.; Jamshad, M.; Parslow, R. A.; Lin, Y. P.; Goldman, A.; Sridhar, P.; Overduin, M.; Muench, S. P.; et al. A Method for Detergent-Free Isolation of 
Membrane Proteins in Their Local Lipid Environment. Nat. Protoc. 2016, 11 (7), 1149-1162.

(93) Rajesh, S.; Knowles, T.; Overduin, M. Production of Membrane Proteins without Cells or Detergents. N. Biotechnol. 2010, 28 (3), 250-254.

(94) Jamshad, M.; Lin, Y.-P.; Knowles, T. J.; Parslow, R. A.; Harris, C.; Wheatley, M.; Poyner, D. R.; Bill, R. M.; Thomas, O. R. T.; Overduin, M.; et al. Surfactant-Free Purification of Membrane Proteins with Intact Native Membrane Environment. Biochem. Soc. Trans. 2011, $39(3), 813-818$.

(95) Alfrey, T.; Lavin, E. The Copolymerization of Styrene and Maleic Anhydride. J. Am. Chem. Soc. 1945, 67 (11), 2044-2045.

(96) Huang, J.; Turner, S. R. Recent Advances in Alternating Copolymers: The Synthesis, Modification, and Applications of Precision Polymers. Polymer. 2017, 572-586.

(97) Matsumura, Y.; Maeda, H. A New Concept for Macromolecular Therapeutics in Cancer Chemotherapy: Mechanism of Tumoritropic Accumulation of Proteins and the Antitumor Agent Smancs. Cancer Res. 1986, 46, 6387-6392.

(98) Greish, K.; Fang, J.; Inutsuka, T.; Nagamitsu, A.; Maeda, H. Macromolecular Therapeutics. Clin. Pharmacokinet. 2003, 42 (13), 1089-1105.

(99) Greish, K.; Nagamitsu, A.; Fang, J.; Maeda, H. Copoly(Styrene-Maleic Acid)-Pirarubicin Micelles: High Tumor-Targeting Efficiency with Little Toxicity. Bioconjug. Chem. 2005, 16 (1), 230-236.

(100) Angelova, N.; Yordanov, G. Nanoparticles of Poly(Styrene-Co-Maleic Acid) as Colloidal Carriers for the Anticancer Drug Epirubicin. Colloids Surfaces A Physicochem. Eng. Asp. 2014, No. 452, 73-81.

(101) Henry, S. M.; El-sayed, M. E. H.; Pirie, C. M.; Hoffman, A. S.; Stayton, P. S. PH-Responsive Poly ( Styrene-Alt-Maleic Anhydride ) Alkylamide Copolymers for Intracellular Drug Delivery. Biomacromolecules 2006, 7 (8), 2407-2414.

(102) SMALP Network - Publications http://www.smalp.net/index.html (accessed Dec 31, 2020).

(103) Stroud, Z.; Hall, S. C. L.; Dafforn, T. R. Purification of Membrane Proteins Free from Conventional Detergents: SMA, New Polymers, New Opportunities and New Insights. Methods 2018, 147, 106-117. 
(104) Sahu, I. D.; Zhang, R.; Dunagan, M. M.; Craig, A. F.; Lorigan, G. A. Characterization of KCNE1 inside Lipodisq Nanoparticles for EPR Spectroscopic Studies of Membrane Proteins. J. Phys. Chem. B 2017, 121 (21), 5312-5321.

(105) Gulati, S.; Jamshad, M.; Knowles, T. J.; Morrison, K. A.; Downing, R.; Cant, N.; Collins, R.; Koenderink, J. B.; Ford, R. C.; Overduin, M.; et al. Detergent-Free Purification of ABC (ATPBinding-Cassette) Transporters. Biochem. J 2014, 461, 269-278.

(106) Prabudiansyah, I.; Kusters, I.; Caforio, A.; Driessen, A. J. M. Characterization of the Annular Lipid Shell of the Sec Translocon. BBA - Biomembr. 2015, 1848, 2050-2056.

(107) Logez, C.; Damian, M.; Legros, C.; Dupré, C.; Guéry, M.; Mary, S.; Wagner, R.; M’Kadmi, C.; Nosjean, O.; Fould, B.; et al. Detergent-Free Isolation of Functional G Protein-Coupled Receptors into Nanometric Lipid Particles. Biochemistry 2016, 55 (1), 38-48.

(108) Smirnova, I. A.; Sjöstrand, D.; Li, F.; Björck, M.; Schäfer, J.; Östbye, H.; Högbom, M.; von Ballmoos, C.; Lander, G. C.; Ädelroth, P.; et al. Isolation of Yeast Complex IV in Native Lipid Nanodiscs. Biochim. Biophys. Acta - Biomembr. 2016, 1858 (12), 2984-2992.

(109) Parmar, M.; Rawson, S.; Scarff, C. A.; Goldman, A.; Dafforn, T. R.; Muench, S. P.; Postis, V. L. G. Using a SMALP Platform to Determine a Sub-Nm Single Particle Cryo-EM Membrane Protein Structure. Biochim. Biophys. Acta - Biomembr. 2018, 1860 (2), 378-383.

(110) Krishnarjuna, B.; Ravula, T.; Ramamoorthy, A. Detergent-Free Extraction, Reconstitution and Characterization of Membrane-Anchored Cytochrome-B5 in Native Lipids. Chem. Commun. 2020, 56 (48), 6511-6514.

(111) Paulin, S.; Jamshad, M.; Dafforn, T. R.; Garcia-Lara, J.; Foster, S. J.; Galley, N. F.; Roper, D. I.; Rosado, H.; Taylor, P. W. Surfactant-Free Purification of Membrane Protein Complexes from Bacteria: Application to the Staphylococcal Penicillin-Binding Protein Complex PBP2/PBP2a. Nanotechnology 2014, 25 (28), 285101.

(112) Li, D.; Li, J.; Zhuang, Y.; Zhang, L.; Xiong, Y.; Shi, P.; Tian, C. Nano-Size Uni-Lamellar Lipodisq Improved in Situ Auto-Phosphorylation Analysis of E.Coli Tyrosine Kinase Using 19F Nuclear Magnetic Resonance. Protein Cell 2015, 6 (3), 229-233.

(113) Skaar, K.; Korza, H. J.; Tarry, M.; Sekyrova, P.; Högbom, M. Expression and Subcellular Distribution of GFP-Tagged Human Tetraspanin Proteins in Saccharomyces Cerevisiae. PLoS One 2015, 10 (7), e0134041. 
(114) Jamshad, M.; Charlton, J.; Lin, Y.-P.; Routledge, S. J.; Bawa, Z.; Knowles, T. J.; Overduin, M.; Dekker, N.; Dafforn, T. R.; Bill, R. M.; et al. G-Protein Coupled Receptor Solubilization and Purification for Biophysical Analysis and Functional Studies, in the Total Absence of Detergent. Biosci. Rep. Biosci. Reports 2015, 35.

(115) Orwick-Rydmark, M.; Lovett, J. E.; Graziadei, A.; Lindholm, L.; Hicks, M. R.; Watts, A. Detergent-Free Incorporation of a Seven-Transmembrane Receptor Protein into Nanosized Bilayer Lipodisq Particles for Functional and Biophysical Studies. Nano Lett. 2012, 12 (9), 4687-4692.

(116) Broecker, J.; Eger, B. T.; Ernst, O. P. Crystallogenesis of Membrane Proteins Mediated by Polymer-Bounded Lipid Nanodiscs. Structure 2017, 25 (2), 384-392.

(117) Dörr, J. M.; Koorengevel, M. C.; Schäfer, M.; Prokofyev, A. V.; Scheidelaar, S.; Van Der Cruijsen, E. A. W.; Dafforn, T. R.; Baldus, M.; Killian, J. A. Detergent-Free Isolation, Characterization, and Functional Reconstitution of a Tetrameric $\mathrm{K}^{+}$Channel: The Power of Native Nanodiscs. Proc. Natl. Acad. Sci. U. S. A. 2014, 111 (52), 18607-18612.

(118) Swainsbury, D. J. K.; Scheidelaar, S.; van Grondelle, R.; Killian, J. A.; Jones, M. R. Bacterial Reaction Centers Purified with Styrene Maleic Acid Copolymer Retain Native Membrane Functional Properties and Display Enhanced Stability. Angew. Chem. Int. Ed. Engl. 2014, 53 (44), 11803-11807.

(119) Scheidelaar, S.; Koorengevel, M. C.; Pardo, J. D.; Meeldijk, J. D.; Breukink, E.; Killian, J. A. Molecular Model for the Solubilization of Membranes into Nanodisks by Styrene Maleic Acid Copolymers. Biophys. J. 2015, 108 (2), 279-290.

(120) Scheidelaar, S.; Koorengevel, M. C.; Pardo, J. D.; Meeldijk, J. D.; Breukink, E.; Killian, J. A. Molecular Model for the Solubilization of Membranes into Nanodisks by Styrene Maleic Acid Copolymers. Biophys. J. 2015, 108 (2), 279-290.

(121) Dörr, J. M.; Scheidelaar, S.; Koorengevel, M. C.; Dominguez, J. J.; Schäfer, M.; van Walree, C. A.; Killian, J. A. The Styrene-Maleic Acid Copolymer: A Versatile Tool in Membrane Research. Eur. Biophys. J. 2016, 45 (1), 3-21.

(122) Cuevas Arenas, R.; Klingler, J.; Vargas, C.; Keller, S. Influence of Lipid Bilayer Properties on Nanodisc Formation Mediated by Styrene/Maleic Acid Copolymers. Nanoscale 2016, 8 (32), $15016-15026$.

(123) Grethen, A.; Glueck, D.; Keller, S. Role of Coulombic Repulsion in Collisional Lipid Transfer 
Among SMA(2:1)-Bounded Nanodiscs. J. Membr. Biol. 2018, 251 (3), 443-451.

(124) Kopf, A. H.; Dörr, J. M.; Koorengevel, M. C.; Antoniciello, F.; Jahn, H.; Killian, J. A. Factors Influencing the Solubilization of Membrane Proteins from Escherichia Coli Membranes by Styrene-Maleic Acid Copolymers. Biochim. Biophys. Acta - Biomembr. 2020, 1862 (2), 183125.

(125) Swainsbury, D. J. K.; Scheidelaar, S.; Foster, N.; Van Grondelle, R.; Killian, J. A.; Jones, M. R. The Effectiveness of Styrene-Maleic Acid (SMA) Copolymers for Solubilisation of Integral Membrane Proteins from SMA-Accessible and SMA-Resistant Membranes. 2017.

(126) Xue, M.; Cheng, L.; Faustino, I.; Guo, W.; Marrink, S. J. Molecular Mechanism of Lipid Nanodisc Formation by Styrene Maleic Acid Copolymers. Biophys. J. 2018, 0 (0).

(127) Orwick Rydmark, M.; Christensen, M. K.; Köksal, E. S.; Kantarci, I.; Kustanovich, K.; Yantchev, V.; Jesorka, A.; Gözen, I. Styrene Maleic Acid Copolymer Induces Pores in Biomembranes. Soft Matter 2019, 15 (39), 7934-7944.

(128) Grethen, A.; Oluwole, A. O.; Danielczak, B.; Vargas, C.; Keller, S. Thermodynamics of Nanodisc Formation Mediated by Styrene/Maleic Acid (2:1) Copolymer. Sci. Rep. 2017, 7 (1).

(129) Barniol-Xicota, M.; Verhelst, S. H. L. Isolation of Intramembrane Proteases in Membrane-like Environments. Biochim. Biophys. Acta - Biomembr. 2020, 1862 (4), 183193.

(130) Orwick, M. C.; Judge, P. J.; Procek, J.; Lindholm, L.; Graziadei, A.; Engel, A.; Gröbner, G.; Watts, A. Detergent-Free Formation and Physicochemical Characterization of Nanosized Lipid-Polymer Complexes: Lipodisq. Angew. Chemie Int. Ed. 2012, 51 (19), 4653-4657.

(131) Shaw, A. W.; McLean, M. A.; Sligar, S. G. Phospholipid Phase Transitions in Homogeneous Nanometer Scale Bilayer Discs. FEBS Lett. 2004, 556 (1-3), 260-264.

(132) Hardin, N. Z.; Ravula, T.; Mauro, G. Di; Ramamoorthy, A. Hydrophobic Functionalization of Polyacrylic Acid as a Versatile Platform for the Development of Polymer Lipid Nanodisks. Small 2019, 15 (9), 1-5.

(133) Dominguez Pardo, J. J.; Dörr, J. M.; Renne, M. F.; Ould-Braham, T.; Koorengevel, M. C.; van Steenbergen, M. J.; Killian, J. A. Thermotropic Properties of Phosphatidylcholine Nanodiscs Bounded by Styrene-Maleic Acid Copolymers. Chem. Phys. Lipids 2017, 208, 58-64.

(134) Dominguez Pardo, J. J.; Dorr, J. M.; Renne, M. F.; Ould-Braham, T.; Koorengevel, M. C.; van Steenbergen, M. J.; Killian, J. A. Thermotropic Properties of Phosphatidylcholine Nanodiscs 
Bounded by Styrene-Maleic Acid Copolymers. Chem. Phys. Lipids 2017, 208 (208), 58-64.

(135) Hazell, G.; Arnold, T.; Barker, R. D.; Clifton, L. A.; Steinke, N. J.; Tognoloni, C.; Edler, K. J. Evidence of Lipid Exchange in Styrene Maleic Acid Lipid Particle (SMALP) Nanodisc Systems. Langmuir 2016, 32 (45), 11845-11853.

(136) Cuevas Arenas, R.; Danielczak, B.; Martel, A.; Porcar, L.; Breyton, C.; Ebel, C.; Keller, S. Fast Collisional Lipid Transfer among Polymer-Bounded Nanodiscs. Sci. Rep. 2017, 7.

(137) Yao, Z.; Li, B.; Wang, W.; Pan, Z. Continuous Thermal Bulk Copolymerization of Styrene and Maleic Anhydride. J. Appl. Polym. Sci. 1999, 73 (5), 615-622.

(138) Klumperman, B. Mechanistic Considerations on Styrene-Maleic Anhydride Copolymerization Reactions. Polymer Chemistry. 2010, pp 558-562.

(139) Klumperman, L. Free Radical Copolymerization of Styrene and Maleic Anhydride: Kinetic Studies at Low and Intermediate Conversion. 1994.

(140) Overduin, M.; Klumperman, B. Advancing Membrane Biology with Poly(Styrene-Co-Maleic Acid)-Based Native Nanodiscs. Eur. Polym. J. 2019, 110 (June 2018), 63-68.

(141) Craig, A. F.; Clark, E. E.; Sahu, I. D.; Zhang, R.; Frantz, N. D.; Sameer Al-Abdul-Wahid, M.; Dabney-Smith, C.; Konkolewicz, D.; Lorigan, G. A. Tuning the Size of Styrene-Maleic Acid Copolymer-Lipid Nanoparticles (SMALPs) Using RAFT Polymerization for Biophysical Studies. 2016.

(142) Bali, A. P.; Sahu, I. D.; Craig, A. F.; Clark, E. E.; Burridge, K. M.; Dolan, M. T.; DabneySmith, C.; Konkolewicz, D.; Lorigan, G. A. Structural Characterization of Styrene-Maleic Acid Copolymer-Lipid Nanoparticles (SMALPs) Using EPR Spectroscopy. Chem. Phys. Lipids 2019, 220, 6-13.

(143) Smith, A. A. A.; Autzen, H. E.; Laursen, T.; Wu, V.; Yen, M.; Hall, A.; Hansen, S. D.; Cheng, Y.; $\mathrm{Xu}, \mathrm{T}$. Controlling Styrene Maleic Acid Lipid Particles through RAFT. Biomacromolecules 2017, 18 (11), 3706-3713.

(144) Yao, Z.; Zhang, J.-S.; Chen, M.-L.; Li, B.-J.; Lu, Y.-Y.; Cao, K. Preparation of Well-Defined Block Copolymer Having One Polystyrene Segment and Another Poly(Styrene-Alt-Maleic Anhydride) Segment with RAFT Polymerization. J. Appl. Polym. Sci. 2011, 121, 1740-1746.

(145) Hall, S. C. L.; Tognoloni, C.; Price, G. J.; Klumperman, B.; Edler, K. J.; Dafforn, T. R.; Arnold, T. Influence of Poly(Styrene- Co -Maleic Acid) Copolymer Structure on the Properties and 
Self-Assembly of SMALP Nanodiscs. Biomacromolecules 2018, 19 (3), 761-772.

(146) Overduin, M.; Esmaili, M. Structures and Interactions of Transmembrane Targets in Native Nanodiscs. SLAS Discov. 2019.

(147) Schmidt, V.; Sturgis, J. N. Modifying Styrene-Maleic Acid Co-Polymer for Studying Lipid Nanodiscs. 2018.

(148) Jin, Z.; Du, L.; Zhang, C.; Sugiyama, Y.; Wang, W.; Palui, G.; Wang, S.; Mattoussi, H. Modification of Poly(Maleic Anhydride)-Based Polymers with H2N-R Nucleophiles: Addition or Substitution Reaction? Bioconjug. Chem. 2019, 30 (3), 871-880.

(149) Trivedi, B. C.; Culbertson, B. M. Maleic Anhydride, Springer S.; Springer, 1982.

(150) Oluwole, A. O.; Danielczak, B.; Meister, A.; Babalola, J. O.; Vargas, C.; Keller, S. Solubilization of Membrane Proteins into Functional Lipid-Bilayer Nanodiscs Using a Diisobutylene/Maleic Acid Copolymer. Angew. Chemie - Int. Ed. 2017, 56 (7), 1919-1924.

(151) Esmaili, M.; Brown, C. J.; Shaykhutdinov, R.; Acevedo-Morantes, C.; Wang, Y. L.; Wille, H.; Gandour, R. D.; Turner, S. R.; Overduin, M. Homogeneous Nanodiscs of Native Membranes Formed by Stilbene-Maleic-Acid Copolymers. Nanoscale 2020, 12 (32), 16705-16709.

(152) Hall, S. C. L.; Tognoloni, C.; Charlton, J.; Bragginton, É. C.; Rothnie, A. J.; Sridhar, P.; Wheatley, M.; Knowles, T. J.; Arnold, T.; Edler, K. J.; et al. An Acid-Compatible Co-Polymer for the Solubilization of Membranes and Proteins into Lipid Bilayer-Containing Nanoparticles. Nanoscale 2018, 10, 10609-10619.

(153) Ravula, T.; Hardin, N. Z.; Ramadugu, S. K.; Cox, S. J.; Ramamoorthy, A. Formation of PHResistant Monodispersed Polymer-Lipid Nanodiscs. Angew. Chemie Int. Ed. 2018, 57 (5), $1342-1345$.

(154) Fiori, M. C.; Jiang, Y.; Altenberg, G. A.; Liang, H. Polymer-Encased Nanodiscs with Improved Buffer Compatibility. Sci. Rep. 2017, 7, 7-10.

(155) Ravula, T.; Hardin, N. Z.; Ramadugu, S. K.; Ramamoorthy, A. PH Tunable and Divalent Metal Ion Tolerant Polymer Lipid Nanodiscs. Langmuir 2017, 33 (40), 10655-10662.

(156) Ravula, T.; Ramadugu, S. K.; Di Mauro, G.; Ramamoorthy, A. Bioinspired, Size-Tunable SelfAssembly of Polymer-Lipid Bilayer Nanodiscs. Angew. Chemie - Int. Ed. 2017, 56 (38), 11466-11470. 
(157) Lindhoud, S.; Carvalho, V.; Pronk, J. W.; Aubin-Tam, M. E. SMA-SH: Modified StyreneMaleic Acid Copolymer for Functionalization of Lipid Nanodiscs. Biomacromolecules 2016, $17(4), 1516-1522$.

(158) Yasuhara, K.; Arakida, J.; Ravula, T.; Ramadugu, S. K.; Sahoo, B.; Kikuchi, J.-I.; Ramamoorthy, A. Spontaneous Lipid Nanodisc Fomation by Amphiphilic Polymethacrylate Copolymers. J Am Chem Soc 2017, 139, 18657-18663.

(159) Xie, Z.; Schendel, S.; Matsuyama, S.; Reed, J. C. Acidic PH Promotes Dimerization of Bcl-2 Family Proteins. Biochemistry 1998, 37 (18), 6410-6418.

(160) O’Keefe, D. O.; Cabiaux, V.; Choe, S.; Eisenberg, D.; Collier, R. J. PH-Dependent Insertion of Proteins into Membranes: B-Chain Mutation of Diphtheria Toxin That Inhibits Membrane Translocation, Glu-349----Lys. Proc. Natl. Acad. Sci. U. S. A. 1992, 89 (13), 6202-6206.

(161) Knowles, T. J.; Finka, R.; Smith, C.; Lin, Y. P.; Dafforn, T.; Overduin, M. Membrane Proteins Solubilized Intact in Lipid Containing Nanoparticles Bounded by Styrene Maleic Acid Copolymer. J. Am. Chem. Soc. 2009, 131 (22), 7484-7485.

(162) Punyamoonwongsa, P.; Tangsongcharoen, W.; Phoungtawee, P.; Tighe, B. PH-Responsive Styrene Maleic Anhydride with Improved Surface Activity. KMUTNB Int. J. Appl. Sci. Technol. 2018, No. July.

(163) Hardin, N. Z.; Kocman, V.; Di Mauro, G. M.; Ravula, T.; Ramamoorthy, A. Metal-Chelated Polymer Nanodiscs for NMR Studies. Angew. Chemie - Int. Ed. 2019, 58 (48), 17246-17250.

(164) Burridge, K. M.; Harding, B. D.; Sahu, I. D.; Kearns, M. M.; Stowe, R. B.; Dolan, M. T.; Edelmann, R. E.; Dabney-Smith, C.; Page, R. C.; Konkolewicz, D.; et al. Simple Derivatization of RAFT-Synthesized Styrene-Maleic Anhydride Copolymers for Lipid Disk Formulations. Biomacromolecules 2020, 21 (3), 1274-1284.

(165) Smith, A. A. A.; Autzen, H. E.; Faust, B.; Mann, J. L.; Muir, B. W.; Howard, S.; Postma, A.; Spakowitz, A. J.; Cheng, Y.; Appel, E. A. Lipid Nanodiscs via Ordered Copolymers. Chem 2020, 1-14.

(166) Simon, K. S.; Pollock, N. L.; Lee, S. C. Membrane Protein Nanoparticles: The Shape of Things to Come. Biochem. Soc. Trans. 2018, 46 (6), 1495-1504.

(167) Tanaka, M.; Hosotani, A.; Tachibana, Y.; Nakano, M.; Iwasaki, K.; Kawakami, T.; Mukai, T. Preparation and Characterization of Reconstituted Lipid-Synthetic Polymer Discoidal 
Particles. Langmuir 2015, 31 (46), 12719-12726.

(168) Morrison, K. A.; Akram, A.; Mathews, A.; Khan, Z. A.; Patel, J. H.; Zhou, C.; Hardy, D. J.; Moore-Kelly, C.; Patel, R.; Odiba, V.; et al. Membrane Protein Extraction and Purification Using Styrene-Maleic Acid (SMA) Copolymer: Effect of Variations in Polymer Structure. Biochem. J. 2016, 473 (23), 4349-4360.

(169) Oluwole, A. O.; Danielczak, B.; Meister, A.; Babalola, J. O.; Vargas, C.; Keller, S. Solubilization of Membrane Proteins into Functional Lipid-Bilayer Nanodiscs Using a Diisobutylene/Maleic Acid Copolymer. Angew. Chemie - Int. Ed. 2017, 56 (7), 1919-1924.

(170) Sun, C.; Benlekbir, S.; Venkatakrishnan, P.; Wang, Y.; Hong, S.; Hosler, J.; Tajkhorshid, E.; Rubinstein, J. L.; Gennis, R. B. Structure of the Alternative Complex III in a Supercomplex with Cytochrome Oxidase. Nature 2018, 557 (7703), 123-126.

(171) Laursen, T.; Borch, J.; Knudsen, C.; Bavishi, K.; Torta, F.; Martens, H. J.; Silvestro, D.; Hatzakis, N. S.; Wenk, M. R.; Dafforn, T. R.; et al. Characterization of a Dynamic Metabolon Producing the Defense Compound Dhurrin in Sorghum. Science (80). 2016, 354 (6314), 890 893.

(172) Rajesh, S.; Knowles, T.; Overduin, M. Production of Membrane Proteins without Cells or Detergents. N. Biotechnol. 2011, 28 (3), 250-254.

(173) Ravula, T.; Ramadugu, S. K.; Di Mauro, G. M.; Ramamoorthy, A. Bioinspired, Size-Tunable Self-Assembly of Polymer-Lipid Bilayer Nanodiscs. Angew. Chemie Int. Ed. 2017, 56, $11466-11470$.

(174) Cuevas Arenas, R.; Klingler, J.; Vargas, C.; Keller, S. Influence of Lipid Bilayer Properties on Nanodisc Formation Mediated by Styrene/Maleic Acid Copolymers. Nanoscale 2016, 8 (32), $15016-15026$.

(175) Hall, S. C. L.; Tognoloni, C.; Price, G. J.; Klumperman, B.; Edler, K. J.; Dafforn, T. R.; Arnold, T. Influence of Poly(Styrene- Co -Maleic Acid) Copolymer Structure on the Properties and Self-Assembly of SMALP Nanodiscs. Biomacromolecules 2018, 19 (3), 761-772.

(176) Zhang, R.; Sahu, I. D.; Bali, A. P.; Dabney-Smith, C.; Lorigan, G. A. Characterization of the Structure of Lipodisq Nanoparticles in the Presence of KCNE1 by Dynamic Light Scattering and Transmission Electron Microscopy. Chem. Phys. Lipids 2017, 203, 19-23.

(177) Martinez, D.; Decossas, M.; Kowal, J.; Frey, L.; Stahlberg, H.; Dufourc, E. J.; Riek, R.; 
Habenstein, B.; Bibow, S.; Loquet, A. Lipid Internal Dynamics Probed in Nanodiscs. ChemPhysChem 2017, 18 (19), 2651-2657.

(178) Puthenveetil, R.; Nguyen, K.; Vinogradova, O. Nanodiscs and Solution NMR: Preparation, Application and Challenges. Nanotechnol Rev 2017, 6 (1), 111-125.

(179) Yamamoto, K.; Gildenberg, M.; Ahuja, S.; Im, S. C.; Pearcy, P.; Waskell, L.; Ramamoorthy, A. Probing the Transmembrane Structure and Topology of Microsomal Cytochrome-P450 by Solid-State NMR on Temperature-Resistant Bicelles. Sci. Rep. 2013, 3.

(180) Ramamoorthy, A.; Wei, Y.; Lee, D. K. PISEMA Solid-State NMR Spectroscopy. In Annual Reports on NMR Spectroscopy; 2004; Vol. 52, 1-52.

(181) Radoicic, J.; Park, S. H.; Opella, S. J. Macrodiscs Comprising SMALPs for Oriented Sample Solid-State NMR Spectroscopy of Membrane Proteins. Biophys. J. 2018, 115 (1), 22-25.

(182) Gayen, A.; Banigan, J. R.; Traaseth, N. J. Ligand-Induced Conformational Changes of the Multidrug Resistance Transporter EmrE Probed by Oriented Solid-State NMR Spectroscopy. Angew. Chemie - Int. Ed. 2013, 52 (39), 10321-10324.

(183) Salnikov, E. S.; Anantharamaiah, G. M.; Bechinger, B. Accepted Manuscript Supramolecular Organization of Apolipoprotein A-I-Derived Peptides within Disc-like Arrangements. Biophys. J. 2018.

(184) Salnikov, E. S.; Sarrouj, H.; Reiter, C.; Aisenbrey, C.; Purea, A.; Aussenac, F.; Ouari, O.; Tordo, P.; Fedotenko, I.; Engelke, F.; et al. Solid-State NMR/Dynamic Nuclear Polarization of Polypeptides in Planar Supported Lipid Bilayers. J. Phys. Chem. B 2015, 119 (46), 14574 14583.

(185) Ravula, T.; Ramadugu, S. K.; Di Mauro, G. M.; Ramamoorthy, A. Bioinspired, Size-Tunable Self-Assembly of Polymer-Lipid Bilayer Nanodiscs. Angew. Chemie Int. Ed. 2017, 56, 1146611470.

(186) Komar, J.; Alvira, S.; Schulze, R. J.; Martin, R.; Lycklama Nijeholt, J. A.; Lee, S. C.; Dafforn, T. R.; Deckers-Hebestreit, G.; Berger, I.; Schaffitzel, C.; et al. Membrane Protein Insertion and Assembly by the Bacterial Holo-Translocon SecYEG-SecDF-YajC-YidC.

(187) Bersch, B.; Dörr, J. M.; Hessel, A.; Killian, J. A.; Schanda, P. Proton-Detected Solid-State NMR Spectroscopy of a Zinc Diffusion Facilitator Protein in Native Nanodiscs. Angew. Chemie Int. Ed. 2017, 56 (9), 2508-2512. 
(188) McDowall, J. S.; Ntai, I.; Hake, J.; Whitley, P. R.; Mason, J. M.; Pudney, C. R.; Brown, D. R. Steady-State Kinetics of $\alpha$-Synuclein Ferrireductase Activity Identifies the Catalytically Competent Species. Biochemistry 2017, 56 (19), 2497-2505.

(189) Ravula, T.; Hardin, N. Z.; Bai, J.; Im, S. C.; Waskell, L.; Ramamoorthy, A. Effect of Polymer Charge on Functional Reconstitution of Membrane Proteins in Polymer Nanodiscs. Chem. Commun. 2018, 54 (69), 9615-9618.

(190) Wolf, J.; Aisenbrey, C.; Harmouche, N.; Raya, J.; Bertani, P.; Voievoda, N.; Süss, R.; Bechinger, B. PH-Dependent Membrane Interactions of the Histidine-Rich Cell-Penetrating Peptide LAH4-L1. Biophys. J. 2017, 113 (6), 1290-1300.

(191) Yasuhara, K.; Arakida, J.; Ravula, T.; Ramadugu, S. K.; Sahoo, B.; Kikuchi, J. I.; Ramamoorthy, A. Spontaneous Lipid Nanodisc Fomation by Amphiphilic Polymethacrylate Copolymers. J. Am. Chem. Soc. 2017, 139 (51), 18657-18663. 


\section{Chapter 2}

Use of Paramagnetic Systems to Speed-up NMR Data Acquisition and for Structural and Dynamic Studies

The content for this chapter is partially included in the following review article:

Di Mauro Giacomo M., Kocman Vojč, Veglia Gianluigi, Ramamoorthy Ayyalusamy, "Use of paramagnetic systems to speed-up NMR data acquisition and for structural and dynamic studies", Solid State Nuclear Magnetic Resonance, 102, 10/2019, 36-46 


\subsection{Introduction}

Nuclear magnetic resonance spectroscopy is a well-established technique that allows the study of a variety of systems ranging from small molecules and macromolecules in solution to crystals, powders, and a variety of supramolecular aggregates, including fibrils and bones, in the solid state ${ }^{1-15}$. Even though NMR can provide a plethora of structural and dynamic information, it suffers from being a relatively insensitive technique due to the small energy difference of the eigenstates of nuclear spins (Figure 2.1), which translates to low population differences and consequently poor signal-to-noise $\operatorname{ratios}^{4,16,17}$.

A)

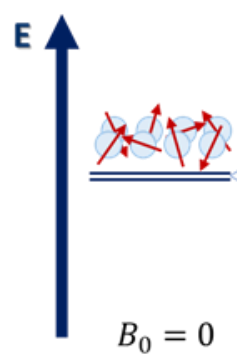

B)

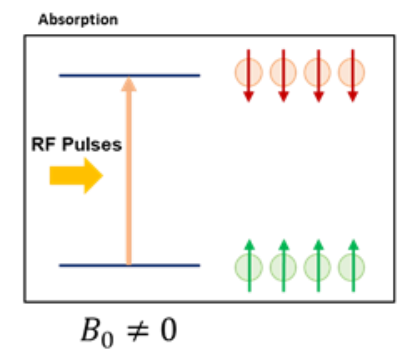

C)

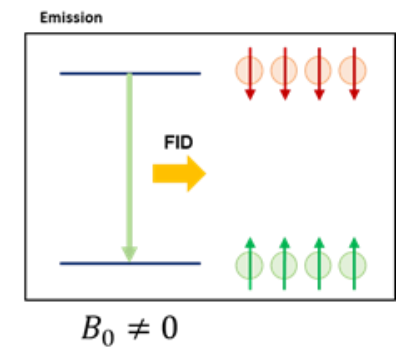

Figure 2.1. The Zeeman effect and fundamentals of NMR spectroscopy. A) Shows the origin of the Zeeman effect. Giving an ensemble of spin-1/2 nuclei, in the absence of an applied magnetic field, all the spins are degenerate. If a magnetic field $\mathrm{B}_{0}$ is applied, the splitting of energy levels is generated, and the energy gap, $\Delta \mathrm{E}$, is proportional to the gyromagnetic ratio and the magnitude of the magnetic field. B) Applying a sequence of RF pulses of comparable energy to the $\Delta \mathrm{E}$, spins are excited, i.e., they flip. C) The free induction decay, FID, is then detected.

Achieving high sensitivity in NMR is additionally hampered by the fact that many of the most common nuclei (such as ${ }^{15} \mathrm{~N}$ and ${ }^{13} \mathrm{C}$ ) studied are low natural-abundance isotopes. As a result, NMR requires samples with high concentrations (in the micro-to-mill-molar range) and volumes of 200- to 300- $\mu \mathrm{L}$ or several milligrams of solid materials. When conducting NMR experiments, especially on biomolecules, it is common to rely on isotopic enrichment, which is both time-consuming and expensive $^{18-22}$. Advancements in probe design and the introduction of cryogenic probes, which enables a significant reduction in noise levels, have also contributed to increased sensitivity of NMR spectra $^{23-25}$. Another way to increase NMR sensitivity, which will be the focus of this chapter, is to affect the relaxation properties of the studied nuclei using paramagnetic probes ${ }^{26-32}$.

Relaxation is a process in which the Boltzmann equilibrium of spin states is regained after the perturbation of nuclear spins by radiofrequency (RF) pulses. Even though the relaxation of multiple- 
spin systems can be a very complex process, often complicated by chemical exchange and local motions, it can be described phenomenologically by longitudinal (or spin-lattice or $\mathrm{T}_{1}$ ) and transverse (or spin-spin or $\mathrm{T}_{2}$ ) relaxations as defined by Bloch equations ${ }^{33}$. The longitudinal relaxation, characterized by the time constant $T_{1}$, describes the energy transfer from the nuclear spin system to the surrounding environment (or lattice). For this process to occur, it is critical that a stochastically fluctuating magnetic field with an appropriate correlation time is present within the system. There are many possible sources for the random fluctuating magnetic fields such as molecular tumbling, internal molecular dynamics, and the effects of free electrons. If intramolecular and tumbling motions are comparable and no free electrons are present, the $\mathrm{T}_{1}$ times of different nuclei within a molecule are closely related to the tumbling rate (also known as the correlation time $\tau_{\mathrm{c}}$ ) of the molecule while in the presence of an external magnetic field. The nuclei with the shortest $T_{1}$ times are in molecules that tumble with a rate that is approximately equal to the Larmor frequency. This means that fastertumbling molecules will have lower $\mathrm{T}_{1}$ values in higher magnetic fields since the Larmor frequency increases with the magnetic field and can more efficiently match the tumbling rate. Importantly, molecules that tumble both faster or slower than the Larmor frequency will relax more slowly due to the mismatch between the tumbling rate and the oscillating fields, and therefore exhibit high $\mathrm{T}_{1}$ values.

Consequently, the $T_{1}$ times of crystals and rigid solids can be quite long (from seconds to even minutes $)^{26}$.

A

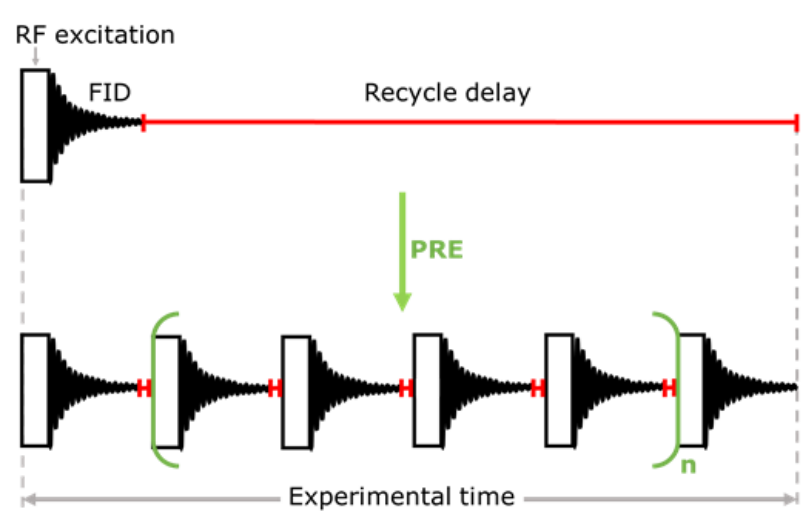

B

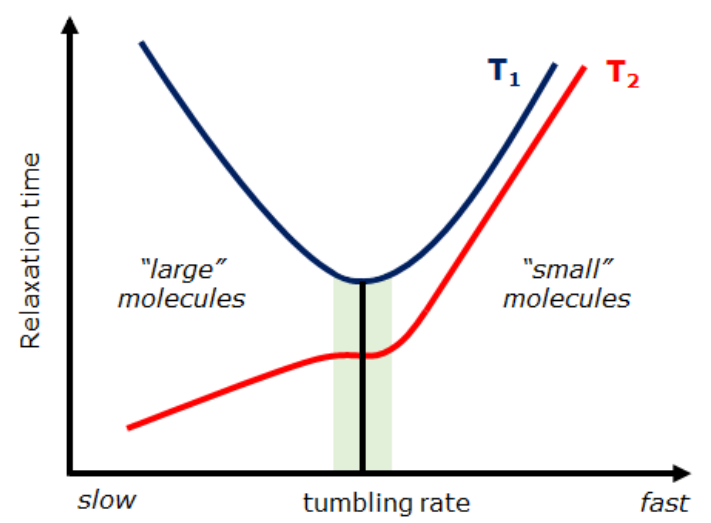

Figure 2.2. PRE and molecular systems. (A) Schematic representation of how acquisition time is affected by the recycle delay. Shorter $T_{1}$ reduces the recycle delay, shortening the experiment. (B) The dependence of relaxation times $\left(\mathrm{T}_{1}\right.$ and $\mathrm{T}_{2}$ ) on the tumbling rate of molecules. This scheme shows that the molecular size plays an active role in both tumbling and relaxation. An optimal range (in light green) is indicated where $\mathrm{T}_{2}$ is not significantly affected by the decreased $\mathrm{T}_{1}$. 
When describing the relaxation processes of a paramagnetic system, it is important to consider the electronic relaxation times, which are mostly dependent on the nature of the chosen paramagnetic ion, the proton effective correlation times (if focused on ${ }^{1} \mathrm{H}$ relaxation), and the molecular rotational correlation time ${ }^{34}$. It was shown that for fast rotating molecules, or part of molecules, the electronic relaxation times dominate, and it is possible to significantly shorten the $T_{1}$ without significantly altering $\mathrm{T}_{2}$.

To improve the sensitivity, NMR spectroscopy relies on signal averaging. However, prior to each scan, the spin states must be brought to thermal equilibrium during the recycle delay time for an optimal signal-to-noise ratio. Consequently, systems with long $\mathrm{T}_{1}$ times are problematic since they require long recycle delays and will significantly prolong the measurement time of an NMR experiment (Figure 2.2A). In addition to $\mathrm{T}_{1}$ time, it is important to also consider the potential loss of transverse magnetization, characterized by the $\mathrm{T}_{2}$ time constant, which leads to the decay of the NMR signal. The $\mathrm{T}_{2}$ times are crucially important for the quality of NMR spectra since the linewidth observed in an NMR spectrum is inversely proportional to $\mathrm{T}_{2}$ of the corresponding nucleus. The $\mathrm{T}_{2}$ times are highly dependent on the nuclei dipole-dipole interaction and large macromolecules with slower correlation times have shorter $\mathrm{T}_{2}$ times. In solid-state samples, the random motion is usually so small that it minimally affects the $\mathrm{T}_{2}$ times of nuclei. Instead, NMR signals are broadened by effects, often classified under $\mathrm{T}_{2}$, , dominated by coherent residuals arising from the incomplete averaging of dipole-dipole interactions by magic-angle spinning and/or decoupling by RF pulses, distributions of chemical shifts arising from heterogeneity, external magnetic field inhomogeneity, or magnetic susceptibility effects ${ }^{35,36}$. Many of these effects can be refocused, albeit not all refocusing is easy to predict, with spin-echo experiments ${ }^{35,37}$. Additionally, decoupling RF pulse sequences were developed specifically to increase coherence lifetimes ${ }^{38,39}$.

A plot of $T_{1}$ and $T_{2}$ dependence in relation to the tumbling rate of molecules exhibit three very distinct regimes as shown in Figure 2.2B: (i) both $\mathrm{T}_{1}$ and $\mathrm{T}_{2}$ is short ("small" molecules regime), (ii) $\mathrm{T}_{1}$ is long, and $\mathrm{T}_{2}$ is short ("large" molecules regime), and (iii) $\mathrm{T}_{1}$ is close or equal $\mathrm{T}_{2}$ (shown in light green). The latter regime of motion is most desirable for NMR spectroscopy since it enables short recycle delays with reasonably narrow resonance lines. In this chapter, we will describe how researchers have created ways to reduce the $T_{1}$ times of nuclei with minimal reductions of their $T_{2}$ times. Our main focus is to describe how to choose and position paramagnetic tags in the systems under investigation and optimize NMR experiments. 


\subsection{Systems for $\mathrm{T}_{1}$ reduction with minimal change on $\mathrm{T}_{2}$}

Different paramagnetic agents have been utilized to reduce the $\mathrm{T}_{1}$ relaxation times in the efforts to shorten the recycle delay times. Paramagnetic probes can be mainly divided into three classes: the nitroxide stable radicals, linear metal chelators, and macrocyclic metal chelators able to bind

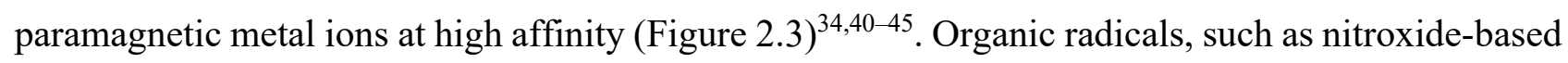
radicals (Figure 2.3A), are commonly used for PRE investigation of different molecular systems. In addition to generating PRE, these radicals are also used as spin labels for Electron Paramagnetic Resonance (EPR) spectroscopy ${ }^{46,47}$ and Dynamic Nuclear Polarization (DNP) studies ${ }^{48,49}$. In addition to free radicals, different metal ions, mostly from the first row of the d-block and the f-block of the periodic table, can be used as paramagnetic probes to explore a variety of paramagnetic effects ${ }^{50-53}$. The paramagnetic properties of metal ions are dictated by their oxidation state, coordination sphere, and electron spin states; therefore, it is possible to use different ligands to fine-tune their properties (Figure 2.3B and 2.3C) $)^{54}$. Importantly, we can observe clear differences in the paramagnetic behavior between the d-block and f-block elements of the periodic table. The d-block elements exhibit several coordination numbers and oxidation states.

Consequently, their electronic and magnetic properties are heavily affected by the type (and number) of coordinating ligands since they interact via their valence electrons where the unpaired electrons are located. The f-block elements form primarily trivalent cations and exhibit high coordination numbers (from 8 to 9). In contrast to d-block, the unpaired electrons of the f-block elements are located in the f-orbitals, which are largely non-bond forming, so the effect of the ligand field on their magnetic properties is much less pronounced. For this reason, it is possible to consider the f-block cations as point charges because any eventual bond with the ligands affects their unpaired electrons only minimally ${ }^{55,56}$.

Favorable electronic relaxation times, proton effective correlation times, and the rotational correlation times of the system can be fine-tuned by varying the ligands coordinated to the paramagnetic metal. In the case of a common chelator such as EDTA (ethylenediaminetetraacetic acid), it has been reported that the $\mathrm{Ni}(\text { EDTA })^{2-}$ complex causes significant line-broadening to the extent that no signals were observable in the ${ }^{1} \mathrm{~N}-{ }^{15} \mathrm{~N}$ HSQC spectra of the studied protein ${ }^{34}$. If, instead, DO2A $(1,7-$ dicarboxymethyl-1,4,7,10-tetraazacyclododecane) is used as a chelating molecule, which forms 1:1 chelates with most transition metals and lanthanide ions, and a strong complex with $\mathrm{Ni}^{2+}$ cations, no significant line-broadening effects, and chemical shift changes were observed for the protein resonances. $\mathrm{Ni}(\mathrm{DO} 2 \mathrm{~A})$ can be used to significantly decrease proton $\mathrm{T}_{1}$ relaxation times of 
macromolecules with negligible line-broadening effects ${ }^{34}$. In contrast, metal ions with slow electronic times, such as $\mathrm{Mn}^{2+}$ and $\mathrm{Gd}^{3+}$ (as well as some organic free radicals, such as TEMPO), decrease the $\mathrm{T}_{2}$ times more significantly, and thereby induce line-broadening ${ }^{57,58}$. Chelators such as EDTA ${ }^{34}$,

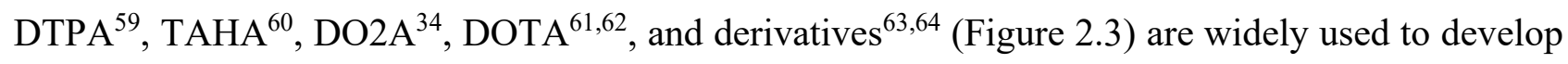
tagging strategies to generate different paramagnetic effects using the desired meta ${ }^{65}$. Additionally, free metals in solution may cause protein structure modification or degradation, and other sample instabilities, e.g., inducing precipitation. In such a case, it is preferable to use paramagnetic metals in their chelated form.

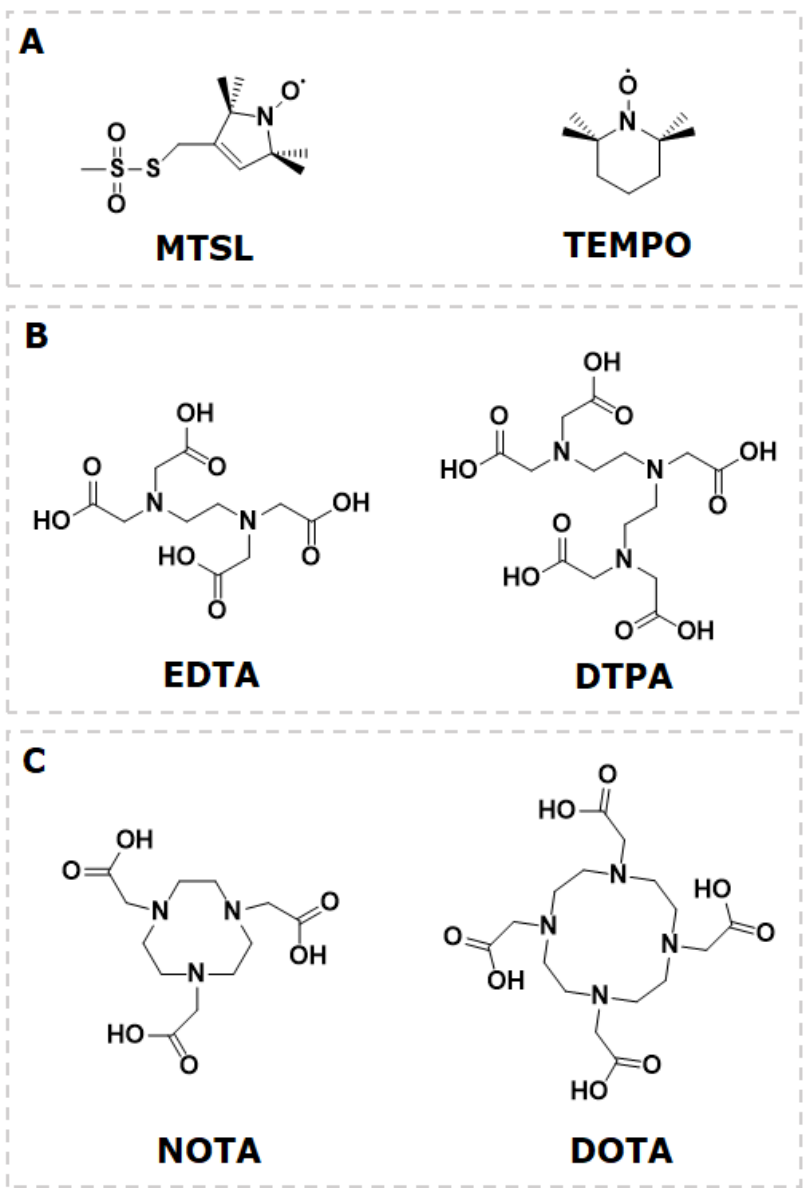

Figure 2.3. Chemical structures of stable free-radicals and metal chelating agents commonly involved in PRE studies. (A) Nitroxide spin labels 1-oxyl-2,2,5,5-tetramethyl-3-pyrroline-3-methyl-methanethiosulfonate (MTSL) and 2,2,6,6-tetramethyl-1-piperidinyloxy (TEMPO). (B) Linear metal chelators ethylenediaminetetraacetic acid (EDTA), and diethylenetriaminepentaacetic acid (DTPA). (C) Macrocyclic metal chelating agents 1,4,7-triazacyclononane1,4,7-triacetic acid (NOTA), and 1,4,7,10-tetraazacyclododecane-1,4,7,10-tetraacetic acid (DOTA). 


\subsection{PRE molecules can be used to obtain structural information}

In addition to expediting the acquisition of NMR spectra, PRE molecules/tags placed at specific

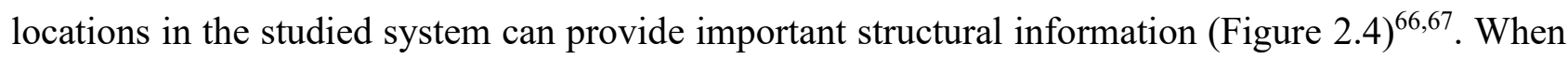
designing the experiment, it is critical to consider that the relaxation effect decreases when moving away from the paramagnetic center with an $r^{-6}$ dependence, where $r$ is the distance between the paramagnetic center and nucleus whose signal is being observed. The region closes to the paramagnetic center, where NMR signals are too broad to be detected, is called the "blind zone." The most useful region is further away from the PRE center, where the NMR signals are visible but still affected by the unpaired electrons that can be used to obtain structural information ${ }^{68,69}$. The sizes of both "blind" and "useful" regions are heavily dependent on the nature of the paramagnetic center. Even by taking the sharp drop-off of the relaxation effects into account, metal ions in paramagnetic molecules can affect nuclei up to a distance of $35 \AA$ due to the large magnetic moments of unpaired electrons.

In biomolecules, paramagnetic probes can be introduced into the sample via simple chemical modifications such as cysteine-cysteine and amide bonds to pre-existing amino acid residues ${ }^{70-72}$ or by adventitious binding to binding sites $^{73}$. However, these methods are often non-trivial and timeconsuming. Examples of paramagnetic tags are S-(1-oxyl-2,2,5,5-tetramethyl-2,5-dihydro- ${ }^{1} \mathrm{H}-$ pyrrol3-yl)methyl-methanesulfono-thioate (MTSL) or Ethylenediaminetetraacetic acid chelated with $\mathrm{Mn}^{2+}$ $\left(\right.$ EDTA-Mn ${ }^{2+}$ ) (Figure 2.3) ${ }^{74}$. If asymmetric isotope labeling is used, as in a system of two interacting proteins, NMR experiments can be designed to distinguish between intra- and inter-molecular PRE effects, which is very useful in the identification of structural changes occurring in both binding partners upon the formation of transient and permanent interactions ${ }^{74,75}$. A paramagnetic tag can be used to detect intermolecular interactions. For instance, a modified phospholipid with a paramagnetic tag in the headgroup will affect only those nuclei that are spatially close to the tag, thus providing information about the orientation of the molecule such as a peptide, protein, or a ligand relative to the lipid head group (Figure 2.4B) ${ }^{76}$.

Additionally, a judicious combination of PRE-tagging and isotope labeling can divulge information about interacting domains of different biomolecules ${ }^{45,74,76,77}$. The simplest way to utilize the PREs effects for structural studies is to dissolve paramagnetic ions into the solution (solvent PREs) that contains macromolecules of interest (Figure 2.4C) ${ }^{77}$. Importantly, the PRE effect is concentrationdependent since multiple PRE centers will increase the relaxation of the exposed nuclei. Paramagnetic ions dissolved in solution with suitable relaxation properties will randomly interact with the 
macromolecules or macromolecular complexes and will effectively suppress the signals of the nuclei that are close enough to the paramagnetic ion ${ }^{26,74}$. On the other hand, signals from chemical groups (amino acid residues or nucleotides) that are shielded from the solvent, and consequently from the paramagnetic ions in solution, can be observed.

For example, $\mathrm{Mn}^{2+}$ ion is commonly used to identify the residues from the transmembrane domains of a membrane protein embedded in a lipid bilayer ${ }^{78,79}$. Solvent PRE is also commonly used to identify the solvent-exposed residues of a protein ${ }^{80,81}$. Additionally, the paramagnetic effect can be used to obtain local structural information by either modifying the molecule under investigation, for instance, proteins, with a conjugated paramagnetic center or by using their innate paramagnetic center (like in the Cytochrome and heme-containing proteins) $)^{26,82-85}$.

A

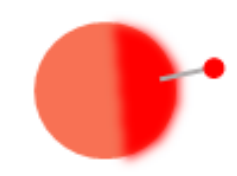

B

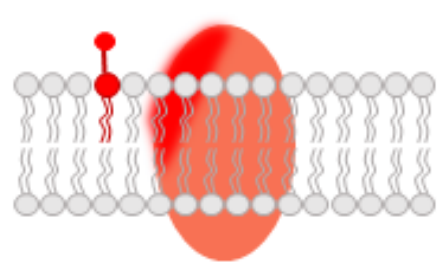

C

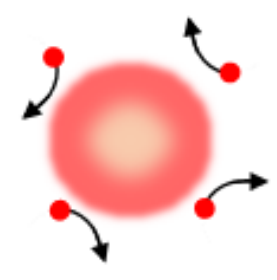

Figure 2.4. Schematic representation of the tagging strategies for PRE studies. (A) Direct or intramolecular labeling, (B) Indirect or intermolecular labeling, (C) Solvent PREs arising from random interactions between a macromolecule and paramagnetic co-solute molecules. Inspired by reference ${ }^{68}$.

\subsection{Solid-state NMR and PRE}

Solid-state NMR spectra get substantially more complicated with the appearance of chemical shift anisotropy, dipolar and quadrupolar couplings, which are otherwise averaged out under isotropic motion of molecules in solution. In addition, very slow or no molecular tumbling in solids, crystals, 
or other viscous and aligned samples that are studied using solid-state NMR spectroscopy usually give rise to long $T_{1}$ and, as discussed above, broad signals due to $T_{2}$ ' effects ${ }^{86,87}$. For these systems, this problem is exacerbated as the $T_{1}$ of the dilute spin $1 / 2$ nuclei are usually long (tens of seconds for ${ }^{13} \mathrm{C}$ in powdered organics and minutes for ${ }^{29} \mathrm{Si}$ in framework silicates ${ }^{88,89}$ ). To deal with long $\mathrm{T}_{1}$ and short $\mathrm{T}_{2}$ times of dilute spins, or insensitive nuclei, cross-polarization (CP) methods ${ }^{88,90-92}$ are commonly used to take advantage of the relatively short $\mathrm{T}_{1}$ of the sensitive nuclei (usually ${ }^{1} \mathrm{H}$ ) in addition to enriching the sensitivity of less sensitive nuclei by the highly-abundant and high- $\gamma$ nuclei like protons. This means that compared to single pulse (i.e., direct detection) experiments conducted on insensitive nuclei, the recycle delays can be shorter when cross-polarization (for example, ${ }^{1} \mathrm{H}$ to ${ }^{15} \mathrm{~N}$ ) is used ${ }^{93-95}$. Therefore, the use of PRE-molecules makes it possible to decrease the $\mathrm{T}_{1}$ times of protons and speeding-up the acquisition of cross-polarization based NMR spectra of the corresponding nuclei in solid-state. Many solid-state NMR studies on crystalline samples have utilized paramagnetic dopants to shorten the $\mathrm{T}_{1}$ of protons ${ }^{94}$. This approach was successfully utilized to either decrease the amount of sample required or shorten the experimental measurement time significantly for studies on amyloid peptides and polymorphic pharmaceutical compounds ${ }^{96-98}$. Using paramagnetic relaxation enhancement, it has also been possible to provide atomic-level insight into the structure and dynamics of the organic matrix (primarily type I collagen) and the mineral surface (primarily poorly crystalline calcium-rich carbonated hydroxyapatite) in bone tissues ${ }^{99,100}$. The intrinsic $\mathrm{T}_{1}$ relaxation times of ${ }^{1} \mathrm{H}$ resonances of different amino acid residues were shortened with the use of copper(II) ions coordinated in $\left[\mathrm{Cu}(\mathrm{II})\left(\mathrm{NH}_{4}\right)_{2} \mathrm{EDTA}\right]^{99,100}$ (Figure 2.5). Importantly, the linewidth was not significantly affected, suggesting that the spin-spin relaxation was not altered in these samples. Shortening $\mathrm{T}_{1}$ allowed for the acceleration of data acquisition from cross-polarization magic-angle-spinning (CP-MAS) NMR experiments, which enabled the use of a natural-abundance ${ }^{13} \mathrm{C}$ bone sample (Figure 2.5).

Furthermore, it was possible to obtain structural information due to the quenching of specific ${ }^{13} \mathrm{C}$ resonances by $\mathrm{Cu}^{2+}$ ions in the absence of mineral. These results showed that three main amino acid residues (glycine, proline, and alanine) from the protein backbone are located close to the bone mineral surface ${ }^{100}$. Simply detecting $\mathrm{T}_{1}$ times for nuclei of different isotopes inside solids can also lead to useful structural and kinetic information. In the case of a X-Cu(II)-HY zeolite (Y = the starting ratio of $n_{\mathrm{Si}} / \mathrm{n}_{\mathrm{Al}}=2.8$ ), where $\mathrm{X}$ represents the number of $\mathrm{Cu}^{2+}$ ions anchored per unit cell, it was possible to identify the preferred binding sites for the $\mathrm{Cu}^{2+}$ ions inside the unit cell ${ }^{101}$. Also, employing in situ PRE MAS NMR technique, it was possible to determine the reaction pathway of catalytic conversion of acetone to hydrocarbons, enabled by the much shorter ${ }^{1} \mathrm{H}$ and ${ }^{13} \mathrm{C} \mathrm{T}_{1}$ times of the zeolite bound acetone molecule ${ }^{101}$. Note that in most solid-state NMR experiments, radio-frequency induced 
heating of the sample is a problem and may denature the expensive isotope-labeled membrane proteins and, in extreme cases, can also damage the probe. Consequently, special care must be given to reduce the sample heating by preparing deuterated samples and using very fast magic angle spinning in combination with specialized low-E NMR probes and RF pulse sequences that utilize low power pulses for magnetization transfer and decupling in order to fully advantage of the reduced recycle delay ${ }^{14,17,96,102-111}$.

\subsection{Biological membranes: bicelles and nanodiscs}

Biological membranes delimit every cell and all its compartments, playing a pivotal role in basic biological processes. The driving principles of bio-membrane formation lie in the amphipathic properties of phospholipids in an aqueous environment but, despite this "simplicity," membranes are made of a plethora of different lipids, polysaccharides, and proteins involved in intricate interactions and equilibria. Membrane component diversity is crucial to maintain stability, function, and integrity of membrane proteins ${ }^{112,113}$. Different aspects of membrane lipids can be studied by both solution and solid-state NMR ${ }^{15,22,114-120}$. Solution state NMR works best when structural studies are being performed on lipids dissolved in organic solvents or also in favorable cases when dispersed in solution. To obtain more biologically relevant structural information about the nature of the membrane phase (lamellar, hexagonal, isotropic, etc.), its order/dynamics (fluid or gel, or liquidordered with cholesterol), and the molecular structure of embedded lipids, it is better to utilize solidstate NMR methodology. When studying transmembrane and receptor proteins, studies have shown the importance to develop NMR techniques that would allow for the characterization of both the solvent-exposed, more dynamic, and lipid bilayer embedded rigid protein domains at the same time ${ }^{62}$. To achieve this, it is crucial to develop excellent membrane-mimicking systems. The most commonly used membrane-model systems are liposomes (SUVs, LUVs, GUVs, MLVs), bicelles, and nanodiscs ${ }^{121}$. The very large differences in size and tumbling rates of the systems are also reflected in $\mathrm{T}_{1}$ and $\mathrm{T}_{2}$ times of lipids and any molecules embedded in those lipids. Liposomes are the simplest bilayer/mimicking system, consisting of lipids that self/assemble into vesicles. Size-tunability is possible by mechanically breaking them down (extrusion or sonication) ${ }^{122}$. Bicelles consist of discshaped phospholipid bilayers surrounded by a rim containing short-chained detergent molecules ${ }^{15,123-}$ ${ }^{130}$. The ratio of lipid to detergent, also known as q-ratio, allows size tunability. Bicelles with large qratio and multilamellar vesicles (MLVs) are more suited to solid-state NMR due to being large systems and having slow tumbling rates ${ }^{15}$. Recently, non-covalent disc-shaped nanoparticles known as nanodiscs have been introduced ${ }^{131,132}$. Several studies have demonstrated how this system 
represents a suitable membrane-mimicking model and have a resemblance to bicelles. These nanodiscs are made of a planar phospholipid bilayer patch surrounded by an amphiphilic belt made up of proteins $\left(\mathrm{MSP}^{131}\right.$ or its derived peptides $\left.{ }^{133}\right)$ or synthetic polymers $\left(\mathrm{SMA}^{132}, \mathrm{DIBMA}^{134}\right.$, $\mathrm{PMA}^{135}, \mathrm{PAA}^{136}$ ) or designed polymers ${ }^{135,137-142}$. These nanodiscs are free from undesirable detergents and also devoid of membrane curvature. Besides their native lipid membrane-like character, the most important property is that the size of peptide-based and polymer-based nanodiscs is tunable by simply varying the lipid:peptide/polymer ratio. Therefore, these nanodiscs satisfy the requirements of solution NMR experiments, whereas macro-nanodiscs ( $>20 \mathrm{~nm}$ diameter) enable the use of solid-state NMR techniques, making them an excellent system to study the structure and dynamics of membrane proteins in a near-native lipid membrane environment ${ }^{139,143}$.

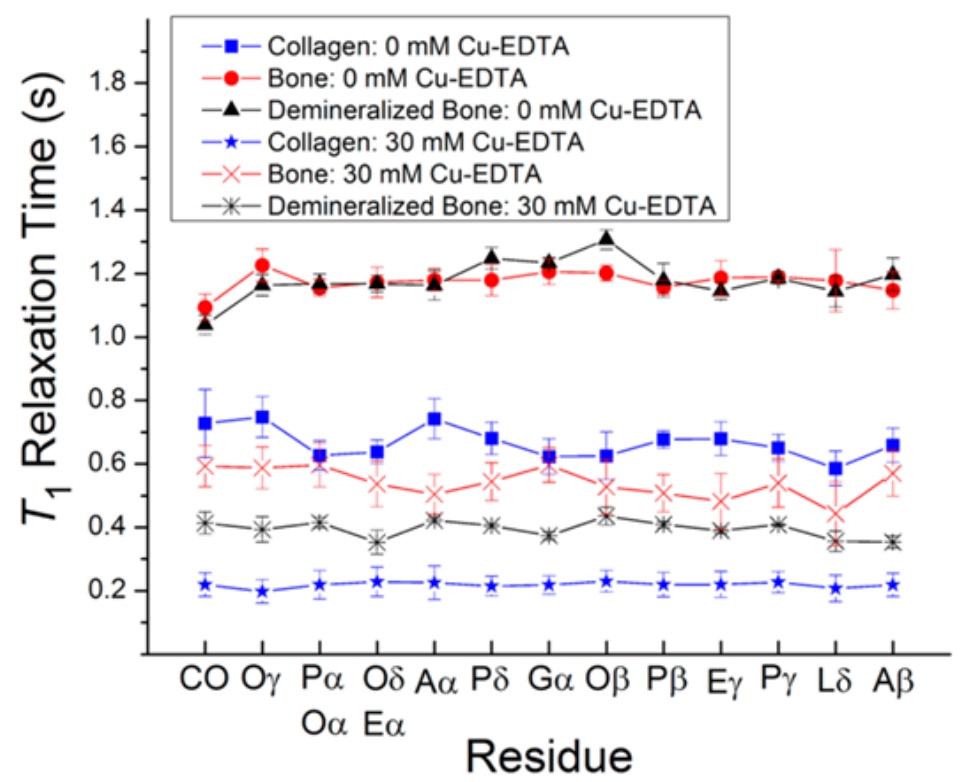

Figure 2.5. $\mathrm{T}_{1}{ }^{1} \mathrm{H}$ relaxation times observed from collagen, powdered cortical bone, and demineralized bone in the absence and in the presence of Cu-EDTA (30 mM). The $\mathrm{T}_{1}$ values were determined from ${ }^{1} \mathrm{H}$-spin-inversion recovery experiments, and the reported errors were estimated from the best-Fitting of experimental data. All measurements were performed on a $600 \mathrm{MHz}$ Bruker AVANCE solid-state NMR spectrometer. A, alanine; L, leucine; P, proline; E, glutamic acid; O, hydroxyproline; G, glycine; CO, carbonyl. The signals from $(\mathrm{P} \alpha, \mathrm{O} \alpha)$ and $(\mathrm{O} \alpha, \mathrm{E} \alpha)$ overlap in the ${ }^{13} \mathrm{C} \mathrm{NMR}$ spectrum. Adapted with permission from ${ }^{100}$. Copyright 2012 American Chemical Society.

As mentioned above, paramagnetic metals have been used to shorten the $\mathrm{T}_{1}$ relaxation of protons in solution and in solid-state NMR experiments to study membrane proteins. Since membrane proteins are embedded in a lipid bilayer, and paramagnetic metals exhibit less PRE effect for residues in the transmembrane region, a higher amount of salt is required to effectively shorten the $T_{1}$ values for the transmembrane residues. However, a high amount of salt can have undesired effects on protein-lipid 
interactions. To avoid this problem, metal-chelated lipids were developed that can be reconstituted in the lipid bilayer sample (Figures 2.6C and 2.6D) ${ }^{45}$. Using a copper-chelated lipid, $\mathrm{T}_{1}$ times of ${ }^{1} \mathrm{H}$ resonances of membrane lipids (Figure 2.6A and 2.6B), membrane-embedded peptides, and proteins were effectively shortened ${ }^{45,76}$. Since the metal-chelated lipid is immobilized within the lipid bilayer, the PRE effect has been shown to be dramatic due to the ${ }^{1} \mathrm{H}-{ }^{1} \mathrm{H}$ dipolar couplings enabled spin diffusion process (Figure 2.7), and therefore the amount of paramagnetic metal ions required to achieve $T_{1}$ reduction was significantly reduced as compared to previous studies that used the paramagnetic metal ions in the bulk solution. An additional benefit is the absence of free metal ions in the samples, which otherwise can induce undesired side effects, as mentioned above.
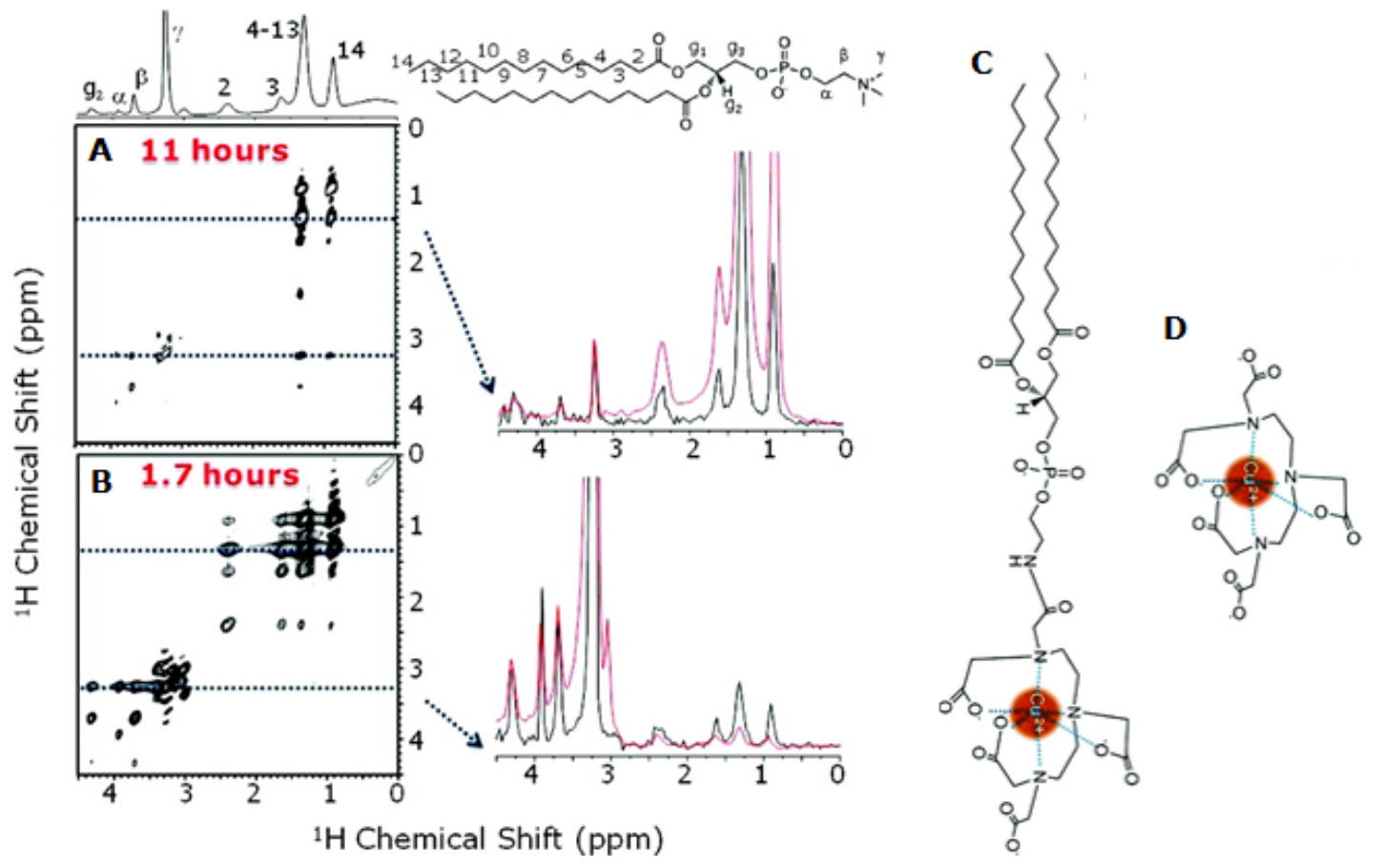

Figure 2.6. $2 \mathrm{D}{ }^{1} \mathrm{H} /{ }^{1} \mathrm{H}$ chemical shift correlation spectra of bicelles without (A) and with (B) $2.56 \mathrm{mM}$ copper-chelated lipid obtained under $5 \mathrm{kHz}$ MAS with total data collection times of 11 and $1.77 \mathrm{~h}$, respectively. A 6.2-fold reduction in data collection time with a similar $\mathrm{S} / \mathrm{N}$ ratio was made possible by the use of the copper-chelated lipid, as can be seen from the 1D spectral slices taken at (top) 1.34 and (bottom) 3.25 ppm with (red) and without (black) the copper-chelating lipid. An RFDR7 sequence with a $100 \mathrm{~ms}$ mixing time and a $100 \mathrm{~ms}$ low-power pulse for water saturation at $35^{\circ} \mathrm{C}$ was used; $512 \mathrm{t} 1$ experiments with 32 scans were used, with recycle delays of $0.2 \mathrm{~s}$ (with copper-chelating lipid) and $2 \mathrm{~s}$ (without). (C) The structure of DMPE-DTPA (1,2-ditetradecanoyl-sn-glycero-3-phosphoethanolamine-Ndiethylenetriaminepentaacetic acid). (D) Molecular structure of DTPA (diethylenetriaminepentaacetic acid) chelated with a copper ion. DTPA is one of the common metal ion chelators. Adapted with permission from ${ }^{76}$. Copyright 2010 American Chemical Society.

Using the specially designed chelating phospholipid (i.e., DMPE-DTPA, ${ }^{45,76}$ ), it is possible to enhance the $T_{1}$ relaxation without affecting the lipid bilayer orientation (Figure 2.7). If isotopically 
tumbling membrane systems that contain metal-chelated lipids are used, as can be done with isotropic bicelles or nanodiscs, the data collection times can be dramatically reduced in solution NMR experiments such as 2D SOFAST-HMQC. As shown in Figures 2.8, it is remarkable that this approach enabled the acquisition of a $2 \mathrm{D}{ }^{1} \mathrm{H} /{ }^{15} \mathrm{~N}$ SOFAST-HMQC spectrum of an antimicrobial peptide without the need for ${ }^{15} \mathrm{~N}$ isotopic enrichment (Figure 2.8A, C, and D) ${ }^{45}$. The used sample preparation approach can be optimized for most membrane proteins to speed-up the acquisition of multidimensional solution NMR experiments for structural and dynamic studies of membrane proteins and dramatically reduce the amount of membrane protein required to acquire NMR spectra.
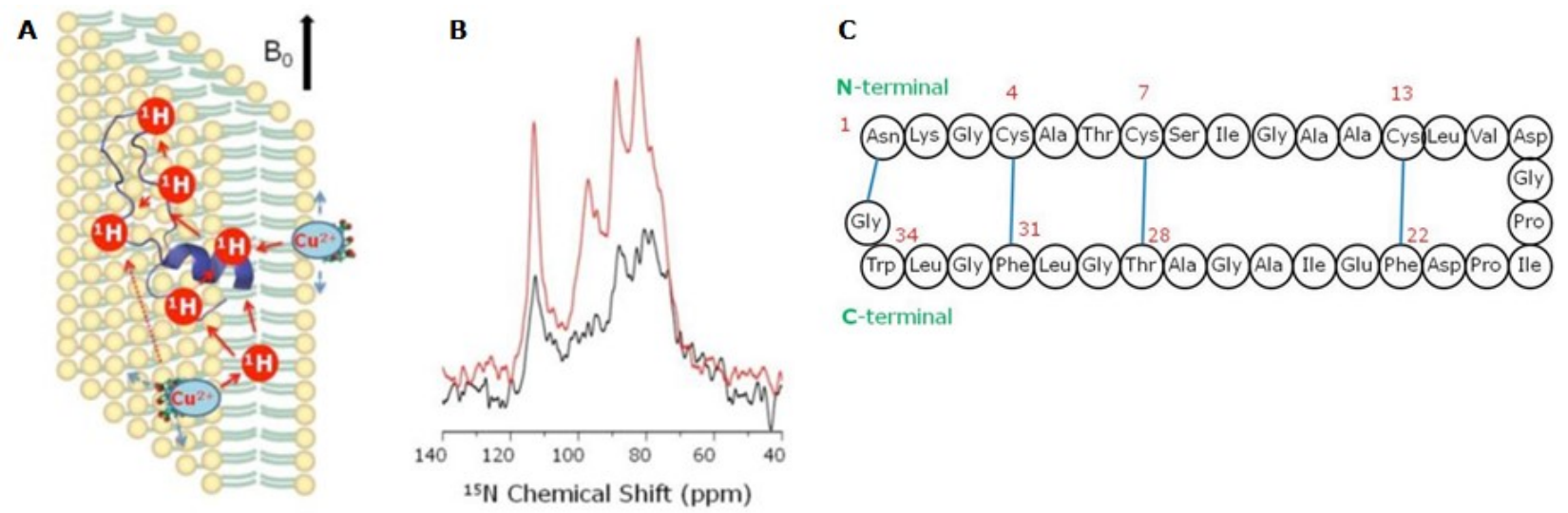

Figure 2.7. (A) Representation of lipid bilayers containing a paramagnetic copper-chelated lipid and Subtilosin A, a 35residue cyclic antimicrobial peptide that has been shown to interact with lipid bilayers with the membrane orientation depicted in (A). (B) ${ }^{15} \mathrm{~N}$ spectra of aligned 7:3 DMPC/DHPC bicelles containing $12-14 \%$ uniformly ${ }^{15} \mathrm{~N}$ labeled (only 70$82 \mathrm{nmol}$ ) Subtilosin A (red) with and (black) without the $2.56 \mathrm{mM}$ copper-chelated lipid. The spectra were obtained on a $400 \mathrm{MHz}$ Varian NMR spectrometer using a ramped-amplitude cross-polarization (ramp-CP) sequence with a contact time of $0.8 \mathrm{~ms}$ under static conditions at $37^{\circ} \mathrm{C}$. While the total data collection time was $8 \mathrm{~h}$ for both spectra, the recycle delay was different for samples without $(2 \mathrm{~s})$ and with $(1 \mathrm{~s})$ the copper-chelated lipid. The transfer of the paramagnetic effect in $T_{1}$ reduction for nuclei in the membrane via proton spin diffusion is also indicated in (A). (C) Primary structure of the antimicrobial peptide Subtilosin A. Adapted with permission from ${ }^{76}$. Copyright 2010 American Chemical Society.

Consequently, this approach is highly beneficial to overcome many intrinsic limitations in membrane protein structural studies. Some of the immediate applications include: (i) quickly acquiring NMR spectra of unstable membrane proteins or protein-protein complexes; (ii) performing NMR experiments on scarcely available mammalian membrane proteins; (iii) real-time monitoring of protein-lipid interactions induced folding, refolding, misfolding, and oligomerization of amyloid proteins; (iv) investigating the action of membrane-active peptides such as antimicrobial peptides and amyloid peptides. 


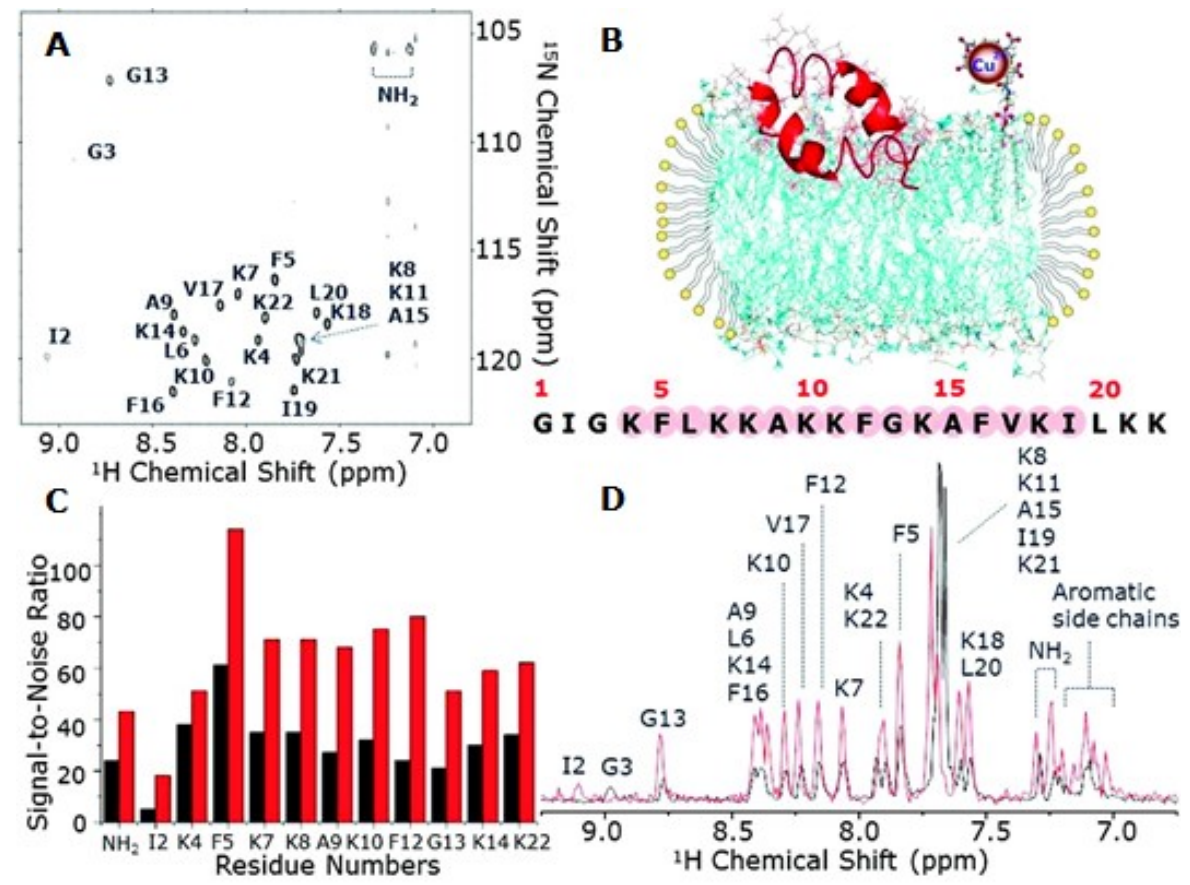

Figure 2.8. Four-fold increase in the sensitivity of 2D SOFAST-HMQC experiments. (A) $2 \mathrm{D}$ SOFAST- ${ }^{1} \mathrm{H} /{ }^{15} \mathrm{~N}-$ HMQC spectrum of a $9.3 \mathrm{mM}$ (unlabeled) MSI-78 (also known as pexiganan) incorporated in DMPC/DHPC isotropic bicelles $(\mathrm{q}=[\mathrm{DMPC}] /[\mathrm{DHPC}]=0.25$, DMPC: 1,2- dimyristoyl-sn-glycero-3-phosphocholine, DHPC: 1,2-dihexanoylsn-glycero-3-phosphocholine) containing a $2.96 \mathrm{mM}$ copper-chelated DMPE lipid. (B) 3D structure of MSI-78 embedded in bicelles along with its amino acid sequence. (C) Signal-to-noise ratio obtained from 2D SOFAST-HMQC spectra of a $9.3 \mathrm{mM}$ unlabeled MSI-78 in $\mathrm{q}=0.5$ isotropic bicelles without copper-chelated lipid (black) and with a $2.96 \mathrm{mM}$ copperchelated lipid (red). (D) $1 \mathrm{D}{ }^{1} \mathrm{H}$ chemical shift projection spectrum obtained from 2D SOFAST- ${ }^{1} \mathrm{H} /{ }^{15} \mathrm{~N}-\mathrm{HMQC}$ spectra that were obtained with no copper (black) and a $2.96 \mathrm{mM} \mathrm{Cu}^{2+}$-DMPE-DTPA (red). All spectra presented in this study were obtained from a $900 \mathrm{MHz}$ Bruker NMR spectrometer at $35{ }^{\circ} \mathrm{C}$ using a cryoprobe. Each 2D SOFAST-HMQC spectrum was obtained from $64 \mathrm{tl}$ experiments, 256 scans, and a $100 \mathrm{~ms}$ recycle delay; the total data collection time (including the acquisition time and delays in INEPT) was $54 \mathrm{~min}$. Adapted with permission from ${ }^{45}$. Copyright 2011 American Chemical Society.

Also useful is the application of solid-state NMR, which is one of the most powerful spectroscopic techniques that enable structural studies of membrane proteins in lipids under liquid crystalline state $^{144-146}$. Many successful relaxation enhancement solid-state NMR experiments carried out on membrane proteins utilizing different paramagnetic metal ions (i.e., $\mathrm{Co}^{3+}, \mathrm{Ni}^{2+}$, and $\mathrm{Gd}^{3+}$ ) have been reported $^{62,147}$. Critically, when discussing the NMR studies of membrane mimetics, it is important to consider alignment since aligned samples can be used to reintroduce anisotropic interactions such as dipolar couplings and chemical shift anisotropy (CSA) into NMR spectra, an invaluable tool to determine the relative orientation of domains in multidomain proteins and solve the high-resolution structure and topology of the protein ${ }^{15,148}$. Determining how proteins orient in a lipid bilayer is crucial for understanding their biological function (Figure 2.9B). Macroscopically aligned lipid bilayer 
samples can be prepared by either using the mechanical alignment of lipids between glass plates or aluminum discs or by magnetically aligning samples ${ }^{148-150}$.

a)

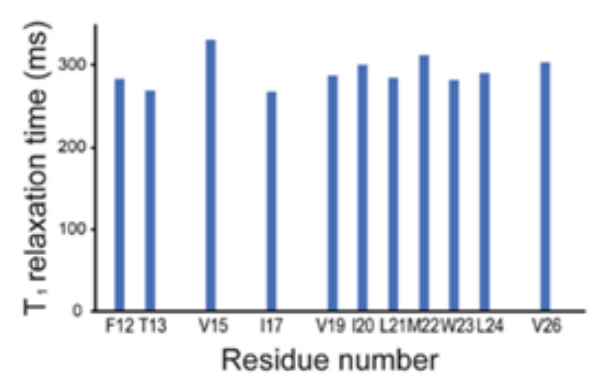

c)

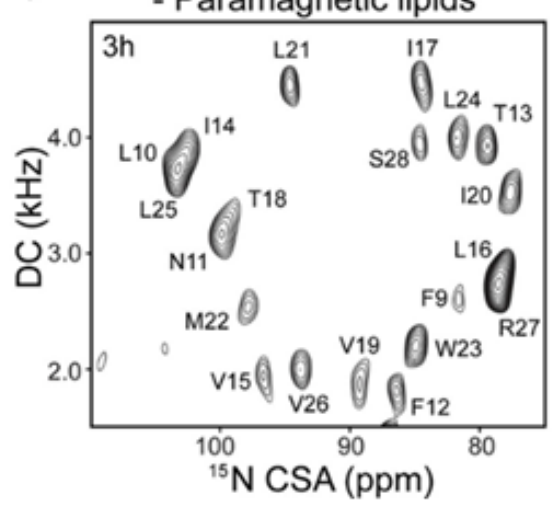

b)

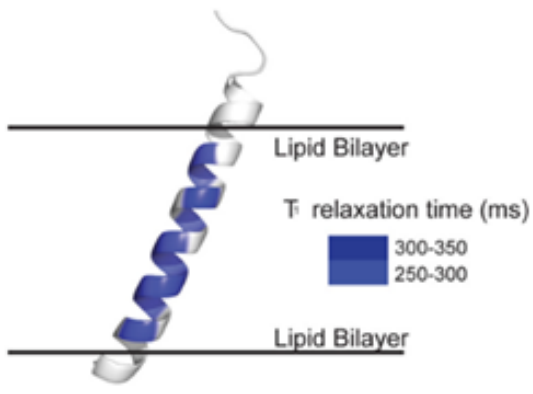

d)

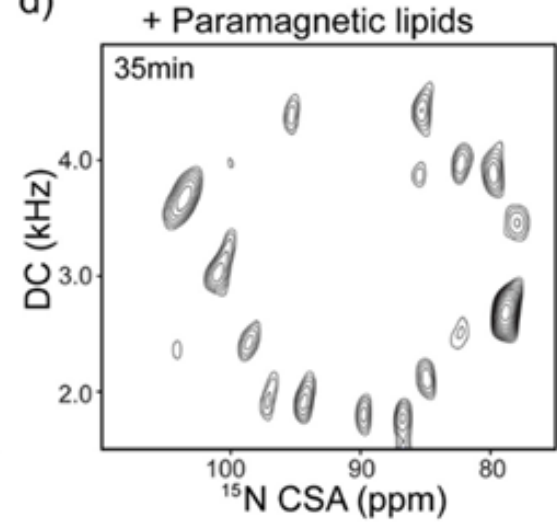

Figure 2.9. Site-specific ${ }^{1} \mathrm{H} \mathrm{T}_{1}$ relaxation times of the backbone resonances of $\left[\mathrm{U}-{ }^{15} \mathrm{~N}\right]-\mathrm{SLN}$ oriented in lipid bicelles with $5 \% \mathrm{Cu}^{2+}$-chelated lipids measured by the 2D inversion recovery SAMPI4 experiment. B) Mapping of the sitespecific $\mathrm{T}_{1}$ relaxation times on SLN structure (blue), where unobservable/overlapped residues in the SAMPI4 spectrum are shown in white. C) 2D SE-SAMPI4 spectra of [U- $\left.{ }^{15} \mathrm{~N}\right]-\mathrm{SLN}$ without paramagnetic and d) with $5 \% \mathrm{Cu}^{2+}$-chelated lipids. The average ${ }^{15} \mathrm{~N}$ linewidths for isolated peaks are $82.9 \mathrm{~Hz}$ and $86.7 \mathrm{~Hz}$ without and with $5 \% \mathrm{Cu}^{2+}$-chelated lipids, respectively. Adapted with permission from ${ }^{151}$. Copyright 2018 Elsevier.

Focusing on magnetic-alignment, it is possible to align lipid bilayers in the form of bicelles or macronanodiscs, as demonstrated in the literature ${ }^{139,146}$. Indeed, if the diameter of these nanoparticles is larger than $20 \mathrm{~nm}$ (called macro-nanodiscs), and their lipid concentration is high enough, they are likely to align in the magnetic field of the spectrometer as successfully demonstrated for many different types of polymer-based nanodiscs ${ }^{139}$. Many different NMR techniques have been developed to monitor the alignment of different membrane systems. For instance, a fast-tumbling lipid bilayer shows a narrow isotropic spectral line. On the other hand, if the bilayer is aligned in an external magnetic field, then the ${ }^{31} \mathrm{P}$ chemical shift is anisotropic. Utilizing alignment, two-dimensional separated-local-field (SLF) experiments that correlate ${ }^{15} \mathrm{~N}$ chemical shift and ${ }^{1} \mathrm{H}^{-15} \mathrm{~N}$ dipolar coupling have been used in the structural studies of membrane proteins and other functional peptides (Figure $2.9)^{151}$. The prototype of such experiments is the polarization inversion by spin-exchange at the magic 
angle (PISEMA) experiment (and its variants) that allows the display of characteristic patterns based on a molecule's orientation with respect to the lipid bilayer (Figure $2.9 \mathrm{C}$ and $2.9 \mathrm{D})^{151-156}$. The socalled helical wheels can be used to infer a helical peptide's tilt within the bilayer, but its analysis requires detailed knowledge of the chemical shift anisotropy tensor within the geometry of an amide bond. Several methods can be used to enhance the sensitivity of these 2D SLF experiments. The copper-chelated lipid developed in the Ramamoorthy lab has also been successfully utilized for the fast acquisition of solid-state NMR spectra in the investigation of structure and membrane orientation of SLN membrane protein (Figure 2.9A). Due to the hydrocarbon chains of the dominant DMPC lipids, which have a negative magnetic susceptibility anisotropy, the bicelles are normally aligned with the bilayer normal perpendicular to the magnetic field direction ${ }^{157}$. Adding paramagnetic lanthanide ions $\left(\mathrm{Yb}^{3+}, \mathrm{Tm}^{3+}\right)$ to the studied membrane systems causes them to bind to the lipid phosphate head groups, which results in a change in their magnetic susceptibility from negative to positive, and consequently alters the tilt angle of bicelles. Such bicelles are characterized as "flipped" and are orientated with the lipid bilayer normal parallel to the main magnetic field ${ }^{157,158}$. It is important to keep this in mind when deciding what kind of paramagnetic system to use for reduction of $\mathrm{T}_{1}$ times of the studied membrane system.

Paramagnetic species have been introduced by either using metal-chelated lipids when forming membrane systems or adding 5-DOXYL stearic acid radical to the studied systems ${ }^{159}$. In addition to increasing the speed of data acquisition the membrane-embedded radicals can also provide structural information since they will reduce the signal intensities of nuclei that are close to the paramagnetic centers near the lipid heads. Using a special chelating phospholipid (i.e., DMPE-DTPA ${ }^{45,76}$ ) it is possible to affect the relaxation without affecting the bilayer orientation.

Similarly, macro-nanodiscs can be successfully aligned in the magnetic field ${ }^{136,137,139,143,160}$. So far, to our knowledge, there is no report in literature about PRE studies using aligned macro-nanodiscs. At this point, several approaches can be followed. One of the straightforward approaches involves the use of paramagnetically labeled phospholipids such as DMPE-DTPE, already reported for bicelles $^{45,76}$, while another approach involves the modification of the polymer belt with paramagnetic probes. In both cases, the different positions of the probes could lead to different results given the fact that the paramagnetic centers lie on different positions of the nanoparticle (lipid-bilayer surface vs. polymeric rim of the nanoparticle).

In addition to free or chelated metal ions, paramagnetic species can be introduced by either using metal-chelated lipids when forming membrane systems or adding 5-DOXYL stearic acid radical to the studied systems ${ }^{159}$. Using paramagnetic ion chelated lipids has an advantage of ion centers being 
located close to the proteins, which increases the PRE effects. The amount of the metal-chelated lipids needed is also very low, only $5-10 \%$, to ensure a significant PRE effect. Lower metal ion concentrations that are used with chelated lipids, compared to metal ions bound to free chelators, are also advantageous since they reduce RF heating of the sample and can consequently allow for shorter recycle delay times. In addition to increasing the speed of NMR data acquisition, the membraneembedded radicals can also provide structural information since they will reduce the magnetization of nuclei that are close to the paramagnetic centers near the lipid head group.

\subsection{Dynamic Nuclear Polarization (DNP) to improve ssNMR}

Paramagnetic dopants can also be effectively utilized in DNP, a process utilized to transfer polarization from the unpaired electron of a stable radical to nuclear spins, enhancing the NMR sensitivity dramatically at cryogenic temperatures ${ }^{49,161-174}$. With the use of stable radicals, it has been shown to be possible to increase the polarization of the studied species by as much as 10,000 times and more at a very low temperature $(\sim 1-2 \mathrm{~K})^{48}$. This is especially useful for DNP-enhanced solidstate NMR applications ${ }^{173,175,176}$. Recently, DNP-enhanced MAS NMR has moved well beyond the proof-of-principle phase, particularly in the field of structural biology and to study materials. Various types of polarizing agents, typically stable organic free radicals, used in DNP have different Electron Paramagnetic Resonance (EPR) spectra, which exhibit different rates of DNP enhancements ${ }^{177}$. For instance, trityl radicals have narrow EPR spectra and are ideal for direct polarization of ${ }^{13} \mathrm{C}$ nuclei, and the polarization process can be additionally enhanced in the presence of PRE compounds ${ }^{178}$. A recent study has shown that PRE additives are capable of increasing the DNP enhancement.

Additionally, it was demonstrated that the reduction in the electron $\mathrm{T}_{1}$ time of the polarizing agent by a PRE compound led to an increase in DNP enhancement. Importantly, a beneficial DNP enhancement is not always connected to shorter electron $\mathrm{T}_{1}$ times but is highly dependent on the studied system. Solid-state ${ }^{13} \mathrm{C}$ DNP signals were enhanced by $100-250 \%$ with the use of trace amounts of paramagnetic additives, with lanthanide complexes with $\mathrm{Gd}^{3+}, \mathrm{Ho}^{3+}, \mathrm{Dy}^{3+}$, and $\mathrm{Tb}^{3+}$ being the most studied ${ }^{173,177,179-181}$. In contrast, $\mathrm{Cu}^{2+}$ - and $\mathrm{Co}^{2+}$-NOTA complexes had virtually no DNP enhancement on ${ }^{13} \mathrm{C}$ signals ${ }^{175}$. It is also possible to directly use paramagnetic metals for sensitivity enhancements of the studied species ${ }^{161}$. This is useful when exogenous radicals, e.g., nitroxides, limit the sensitivity enhancement to the surface and sub-surface layers of the particles, such as the case in the study of inorganic particles and non-porous materials. Additionally, the high reactivity of radicals can also be an issue when studying solid samples. Recently, it was shown that $\mathrm{Mn}^{2+}$ and $\mathrm{Gd}^{3+}$ dopants 
are able to polarize ${ }^{7} \mathrm{Li}$ nuclei in the bulk of micron-sized LTO ( $\mathrm{Li}_{4} \mathrm{Ti}_{5} \mathrm{O}_{12}$ spinel) particles with the use of DNP ${ }^{161}$. Compared to DNP experiments conducted with stable radicals, the enhancements are low, with enhancement factors ranging from 3 to 14, but still quite significant since they can translate even to two orders of magnitude reduction in experimental time ${ }^{161}$. Chelated paramagnetic metal ions were also used in DNP experiments on biomolecules and molecular crystals ${ }^{180,182}$.

Interestingly, applications of paramagnetic metals are also possible in dissolution DNP, where metals such as lanthanides, can be used to increase the polarization in solid-state, but can, due to their relaxation properties, significantly decrease liquid-state polarization ${ }^{172}$. This relaxation by paramagnetic metals can be minimized by chelation of the metal during the dissolution as was shown in the case of chelation of $\mathrm{Gd}^{3+}$ ions by diethylenetriaminepentaacetic acid (DTPA) during the dissolution process. $\mathrm{Gd}^{3+}$ ions could not reduce the $\mathrm{T}_{1}$ of the ${ }^{13} \mathrm{C}$ of the pyruvate molecule in the liquid state due to the chelation with DTPA that increases $\mathrm{Gd}^{3+}{ }^{13} \mathrm{C}$ distance due both to electrostatic repulsion and steric hinderance.

\subsection{Conclusions and future perspectives}

Doping NMR samples with different paramagnetic relaxation enhancement (PRE) agents is an effective way to selectively or uniformly reduce the $T_{1}$ relaxation times of molecules, chemical groups, or species in a variety of samples, including nanomaterials and biological compounds. As demonstrated successfully for many different systems, this approach has been well utilized in speeding-up the acquisition of NMR spectra recorded both with solution and solid samples. In fact, it has become routine to use this approach to acquire solid-state NMR of crystalline of polymorphic pharmaceutical compounds/drugs, amyloid fibers, and membrane proteins. Bones, nanocomposites, and a variety of different nanomaterials benefit from the use of paramagnetism in solid-state NMR. Aspects such as spin-lattice relaxation shortening, or the chance of selectively detecting certain molecular constituents, boost the utility of solid-state NMR spectroscopy for studying nonsoluble/non-crystallizable systems.

It is important to pay special attention to the choice of the paramagnetic center (i.e., radicals vs. metal ions with the optimal chelating agent) to ensure that the PRE probes decrease the $T_{1}$ times without affecting significantly the $\mathrm{T}_{2}$ times of the molecules under investigation. Since studies on functionalizing biomolecules with PRE agents are advancing, further developments to obtain structural and dynamic information and even real-time monitoring of the kinetics of chemical reactions are now feasible. Paramagnetic metals have also been shown to be important in dynamic 
nuclear polarization (DNP) coupled to magic angle spinning (MAS) NMR spectroscopy since they can offer additional sensitivity enhancement and sometimes even be a source of DNP polarization. In fact, paramagnetic DNP MAS NMR spectroscopy can enable structural studies on samples that were unreachable before due to the low abundance of NMR active nuclei.

The use of paramagnetism in solid-state NMR is challenging when applied to different membrane mimetics and used in magnetically aligned samples since paramagnetic agents can also be used to magnetically-align and also alter the alignment direction of membrane-mimicking systems and other biomolecules in the presence of an external magnetic field. Different functionalization of membrane mimetic systems, such as bicelles and nanodiscs, can provide a way to control the position of paramagnetic centers without the introduction of "free" or direct labels to the system of interest (reconstituted membrane proteins, for example). This has the potential, together with the desired alignment, to provide crucial biologically relevant structural information using NMR spectroscopy. Such systems would also enable the applications of a combination of NMR and EPR experiments. 


\section{References}

(1) Ashbrook, S. E.; Griffin, J. M.; Johnston, K. E. Annual Review of Analytical Chemistry Recent Advances in Solid-State Nuclear Magnetic Resonance Spectroscopy. Annu. Rev. Anal. Chem. 2018, 11, 485-508.

(2) Molugu, T. R.; Lee, S.; Brown, M. F. Concepts and Methods of Solid-State NMR Spectroscopy Applied to Biomembranes. Chem. Rev. 2017, 117 (19), 12087-12132.

(3) Xu, J.; Zhu, P.; Gan, Z.; Sahar, N.; Tecklenburg, M.; Morris, M. D.; Kohn, D. H.; Ramamoorthy, A. Natural-Abundance ${ }^{43}$ Ca Solid-State NMR Spectroscopy of Bone. J. Am. Chem. Soc. 2010, 132, 11504-11509.

(4) Opella, S. J.; Marassi, F. M. Structure Determination of Membrane Proteins by NMR Spectroscopy. Chem. Rev. 2004, 104, 3587-3606.

(5) Lacabanne, D.; Fogeron, M. L.; Wiegand, T.; Cadalbert, R.; Meier, B. H.; Böckmann, A. Protein Sample Preparation for Solid-State NMR Investigations. Prog. Nucl. Magn. Reson. Spectrosc. 2019, 110, 20-33.

(6) Stanek, J.; Andreas, L. B.; Jaudzems, K.; Cala, D.; Lalli, D.; Bertarello, A.; Schubeis, T.; Akopjana, I.; Kotelovica, S.; Tars, K.; et al. NMR Spectroscopic Assignment of Backbone and Side-Chain Protons in Fully Protonated Proteins: Microcrystals, Sedimented Assemblies, and Amyloid Fibrils. Angew. Chemie - Int. Ed. 2016, 55, 15504-15509.

(7) Aisenbrey, C.; Salnikov, E. S.; Bechinger, B. Solid - State NMR Investigations of the MHC II Transmembrane Domains: Topological Equilibria and Lipid Interactions. J. Membr. Biol. 2019, No. 0123456789.

(8) Struppe, J.; Quinn, C. M.; Lu, M.; Wang, M.; Hou, G.; Lu, X.; Kraus, J.; Andreas, L. B.; Stanek, J.; Lalli, D.; et al. Expanding the Horizons for Structural Analysis of Fully Protonated Protein Assemblies by NMR Spectroscopy at MAS Frequencies above $100 \mathrm{KHz}$. Solid State 
Nucl. Magn. Reson. 2017, 87, 117-125.

(9) Quinn, C. M.; Wang, M.; Polenova, T. NMR of Macromolecular Assemblies and Machines at $1 \mathrm{GHz}$ and Beyond: New Transformative Opportunities for Molecular Structural Biology BT - Protein NMR: Methods and Protocols; Ghose, R., Ed.; Springer New York: New York, NY, 2018; $1-35$.

(10) Xu, Y.; Zhang, D.; Rogawski, R.; Nimigean, C. M.; Mcdermott, A. E. Identifying Coupled Clusters of Allostery Participants through Chemical Shift Perturbations. PNAS 2019, 116 (6), 2078-2085.

(11) Barbet-Massin, E.; van der Sluis, E.; Musial, J.; Beckmann, R.; Reif, B. Reconstitution of Isotopically Labeled Ribosomal Protein L29 in the 50S Large Ribosomal Subunit for SolutionState and Solid-State NMR BT - Protein Complex Assembly: Methods and Protocols. In Protein Complex Assembly; Marsh, J. A., Ed.; Springer New York: New York, NY, 2018; 87100.

(12) Judge, P. J.; Watts, A. Recent Contributions from Solid-State NMR to the Understanding of Membrane Protein Structure and Function. Curr. Opin. Chem. Biol. 2011, 15, 690-695.

(13) Lisi, G. P.; Loria, J. P. Solution NMR Spectroscopy for the Study of Enzyme Allostery. Chem. Rev. 2016, 116, 6323-6369.

(14) Emsley, L.; Bertini, I. Frontiers in Solid-State NMR Technology. Acc. Chem. Res. 2013, 46 (9), 1912-1913.

(15) Dürr, U. H. N.; Gildenberg, M.; Ramamoorthy, A. The Magic of Bicelles Lights up Membrane Protein Structure. Chem. Rev. 2012, 112 (11), 6054-6074.

(16) Fujiwara, T.; Ramamoorthy, A. How Far Can the Sensitivity of NMR Be Increased? Annu. Reports NMR Spectrosc. 2006, 58, 155-175.

(17) Lee, J. H.; Okuno, Y.; Cavagnero, S. Sensitivity Enhancement in Solution NMR: Emerging Ideas and New Frontiers. J. Magn. Reson. 2014, 241, 18-31.

(18) Verardi, R.; Traaseth, N. J.; Masterson, L. R.; Vostrikov, V. V; Veglia, G. Isotope Labeling for Solution and Solid-State NMR Spectroscopy of Membrane Proteins. In Isotope labeling in Biomolecular NMR; Atreya, H. S., Ed.; Springer Netherlands: Dordrecht, 2012; pp 35-62.

(19) Tugarinov, V.; Kanelis, V.; Kay, L. E. Isotope Labeling Strategies for the Study of HighMolecular-Weight Proteins by Solution NMR Spectroscopy. Nat. Protoc. 2006, 1 (2), 749 
754.

(20) Lacabanne, D.; Meier, B. H.; Böckmann, A. Selective Labeling and Unlabeling Strategies in Protein Solid-State NMR Spectroscopy. J. Biomol. NMR 2018, 71 (3), 141-150.

(21) Hong, M. Resonance Assignment of ${ }^{13} \mathrm{C} /{ }^{15} \mathrm{~N}$ Labeled Solid Proteins by Two- and ThreeDimensional Magic-Angle-Spinning NMR. J. Biomol. NMR 1999, 15 (1), 1-14.

(22) Grage, S. L.; Xu, X.; Schmitt, M.; Wadhwani, P.; Ulrich, A. S. 19F-Labeling of Peptides Revealing Long-Range NMR Distances in Fluid Membranes. J. Phys. Chem. Lett. 2014, 5, 4256-4259.

(23) Mompeán, M.; Sánchez-Donoso, R. M.; De La Hoz, A.; Saggiomo, V.; Velders, A. H.; Gomez, M. V. Pushing Nuclear Magnetic Resonance Sensitivity Limits with Microfluidics and PhotoChemically Induced Dynamic Nuclear Polarization. Nat. Commun. 2018, 9, 1-8.

(24) Charlton, A. J.; Donarski, J. A.; Jones, S. A.; May, B. D.; Clive Thompson, K. The Development of Cryoprobe Nuclear Magnetic Resonance Spectroscopy for the Rapid Detection of Organic Contaminants in Potable Water. J. Environ. Monit. 2006, 8 (11), 11061110 .

(25) Dalisay, D. S.; Molinski, T. F. NMR Quantitation of Natural Products at the Nanomole Scale. J. Nat. Prod. 2009, 72 (4), 739-744.

(26) Pell, A. J.; Pintacuda, G.; Grey, C. P. Paramagnetic NMR in Solution and the Solid State. Prog. Nucl. Magn. Reson. Spectrosc. 2019, 111, 1-271.

(27) Feintuch, A.; Otting, G.; Goldfarb, D. Gd $^{3+}$ Spin Labeling for Measuring Distances in Biomacromolecules: Why and How? Electron Paramagn. Reson. Investig. Biol. Syst. by Using Spin Labels, Spin Probes, Intrinsic Met. Ions, Part A 2015, 563, 415-457.

(28) Potapov, A.; Yagi, H.; Huber, T.; Jergic, S.; Dixon, N. E.; Otting, G.; Goldfarb, D. NanometerScale Distance Measurements in Proteins Using Gd3+ Spin Labeling. J. Am. Chem. Soc. 2010, 132, 9040-9048.

(29) Pintacuda, G.; John, M.; Su, X. C.; Otting, G. NMR Structure Determination of Protein Ligand Complexes by Lanthanide Labeling. Acc. Chem. Res. 2007, 40 (3), 206-212.

(30) Orton, H. W.; Kuprov, I.; Loh, C. T.; Otting, G. Using Paramagnetism to Slow Down Nuclear Relaxation in Protein NMR. J. Phys. Chem. Lett. 2016, 7 (23), 4815-4818. 
(31) Otting, G. Protein NMR Using Paramagnetic Ions. Annu. Rev. Biophys. 2010, 39, 387-405.

(32) Jaroniec, C. P. Solid-State Nuclear Magnetic Resonance Structural Studies of Proteins Using Paramagnetic Probes. Solid State Nuclear Magnetic Resonance. May 2012, pp 1-13.

(33) Bloch, F. Nuclear Induction. Phys. Rev. 1946, 70 (7), 460-474.

(34) Cai, S.; Seu, C.; Kovacs, Z.; Sherry, A. D.; Chen, Y. Sensitivity Enhancement of Multidimensional NMR Experiments by Paramagnetic Relaxation Effects. J. Am. Chem. Soc. 2006, 128 (41), 13474-13478.

(35) De Paëpe, G.; Giraud, N.; Lesage, A.; Hodgkinson, P.; Böckmann, A.; Emsley, L. Transverse Dephasing Optimized Solid-State NMR Spectroscopy. J. Am. Chem. Soc. 2003, 125 (46), $13938-13939$.

(36) Vanderhart, D. L.; Earl, W. L.; Garroway, A. N. Resolution in ${ }^{13}$ C NMR of Organic Solids Using High-Power Proton Decoupling and Magic-Angle Sample Spinning. J. Magn. Reson. 1981, 44 (2), 361-401.

(37) Hahn, E. L. Spin Echoes. Phys. Rev. 1950, 80 (4), 580-594.

(38) Mehring, M. Principles of High Resolution NMR in Solids, Second, Re.; Spring-Verlag, Ed.; Springer, Berlin, Heidelberg: New York, NY, 1983.

(39) Haeberlen, U. High Resolution NMR in solids selective averaging: supplement 1 Advances in Magnetic Resonance; Academic Press, 1976.

(40) Tesch, D. M.; Nevzorov, A. A. Sensitivity Enhancement and Contrasting Information Provided by Free Radicals in Oriented-Sample NMR of Bicelle-Reconstituted Membrane Proteins. $J$. Magn. Reson. 2014, 239, 9-15.

(41) Schlagnitweit, J.; Tang, M.; Baias, M.; Richardson, S.; Schantz, S.; Emsley, L. Nanostructure of Materials Determined by Relayed Paramagnetic Relaxation Enhancement. J. Am. Chem. Soc. 2015, 137 (39), 12482-12485.

(42) Li, W.; Celinski, V. R.; Weber, J.; Kunkel, N.; Kohlmann, H. Homogeneity of Doping with Paramagnetic Ions by NMR. Phys. Chem. Chem. Phys 2016, 18, 9752.

(43) Maron, S.; Dantelle, G.; Gacoin, T.; Devreux, F. NMR and ESR Relaxation in Nd- and GdDoped LaPO4: Towards the Accurate Determination of the Doping Concentration. Phys. Chem. Chem. Phys. 2014, 16 (35), 18788-18798. 
(44) Lo, A. Y. H.; Sudarsan, V.; Sivakumar, S.; Van Veggel, F.; Schurko, R. W. Multinuclear SolidState NMR Spectroscopy of Doped Lanthanum Fluoride Nanoparticles. J. Am. Chem. Soc. 2007, 129 (15), 4687-4700.

(45) Yamamoto, K.; Vivekanandan, S.; Ramamoorthy, A. Fast NMR Data Acquisition from Bicelles Containing a Membrane-Associated Peptide at Natural-Abundance. J. Phys. Chem. B 2011, 115 (43), 12448-12455.

(46) Subczynski, W. K.; Widomska, J.; Feix, J. B. Physical Properties of Lipid Bilayers from EPR Spin Labeling and Their Influence on Chemical Reactions in a Membrane Environment. Free Radic. Biol. Med. 2009, 46 (6), 707-718.

(47) Hauenschild, T.; Hinderberger, D. A Platform of Phenol-Based Nitroxide Radicals as an "EPR Toolbox" in Supramolecular and Click Chemistry. Chempluschem 2019, 84 (1), 43-51.

(48) Ardenkjær-Larsen, J. H.; Fridlund, B.; Gram, A.; Hansson, G.; Hansson, L.; Lerche, M. H.; Servin, R.; Thaning, M.; Golman, K. Increase in Signal-to-Noise Ratio of > 10,000 Times in Liquid-State NMR. Proc. Natl. Acad. Sci. U. S. A. 2003, 100 (18), 10158-10163.

(49) Corzilius, B.; Andreas, L. B.; Smith, A. A.; Ni, Q. Z.; Griffin, R. G. Paramagnet Induced Signal Quenching in MAS-DNP Experiments in Frozen Homogeneous Solutions. J. Magn. Reson. 2014, 240, 113-123.

(50) Pintacuda, G.; Kaikkonen, A.; Otting, G. Modulation of the Distance Dependence of Paramagnetic Relaxation Enhancements by CSA $\times$ DSA Cross-Correlation. J. Magn. Reson. 2004, 171, 233-243.

(51) Nadaud, P. S.; Helmus, J. J.; Kall, S. L.; Jaroniec, C. P. Paramagnetic Ions Enable Tuning of Nuclear Relaxation Rates and Provide Long-Range Structural Restraints in Solid-State NMR of Proteins. J. Am. Chem. Soc. 2009, 131 (23), 8108-8120.

(52) Kurland, R. J.; McGarvey, B. R. Isotropic NMR Shifts in Transition Metal Complexes: The Calculation of the Fermi Contact and Pseudocontact Terms. J. Magn. Reson. 1970, 2 (3), 286301.

(53) Mcconnell, H. M.; Robertson, R. E. Isotropic Nuclear Resonance Shifts. J. Chem. Phys. 1958, $29(6), 1361-1365$.

(54) Luchinat, C.; Parigi, G.; Ravera, E. Paramagnetism in Experimental Biomolecular NMR; 2018.

(55) Bertini, I.; Luchinat, C.; Parigi, G.; Ravera, E. Lanthanoids and Actinoids: Shift and 
Relaxation. In Solution NMR of Paramagnetic Molecules; Elsevier, 2016; 255-276.

(56) Bleaney, B. Nuclear Magnetic Resonance Shifts in Solution Due to Lanthanide Ions. J. Magn. Reson. 1972, 8, 91-100.

(57) Hiller, S.; Wider, G.; Etezady-Esfarjani, T.; Horst, R.; Wüthrich, K. Managing the Solvent Water Polarization to Obtain Improved NMR Spectra of Large Molecular Structures. J. Biomol. NMR 2005, 32 (1), 61-70.

(58) Nitsche, C.; Otting, G. Pseudocontact Shifts in Biomolecular NMR Using Paramagnetic Metal Tags. Prog. Nucl. Magn. Reson. Spectrosc. 2017, 98-99, 20-49.

(59) Mroue, K. H.; Zhang, R.; Zhu, P.; McNerny, E.; Kohn, D. H.; Morris, M. D.; Ramamoorthy, A. Acceleration of Natural-Abundance Solid-State MAS NMR Measurements on Bone by Paramagnetic Relaxation from Gadolinium-DTPA. J. Magn. Reson. 2014, 244, 90-97.

(60) Peters, F.; Maestre-Martinez, M.; Leonov, A.; Kovačič, L.; Becker, S.; Boelens, R.; Griesinger, C. Cys-Ph-TAHA: A Lanthanide Binding Tag for RDC and PCS Enhanced Protein NMR. $J$. Biomol. NMR 2011, 51 (3), 329-337.

(61) Strickland, M.; Schwieters, C. D.; Göbl, C.; Opina, A. C. L.; Strub, M. P.; Swenson, R. E.; Vasalatiy, O.; Tjandra, N. Characterizing the Magnetic Susceptibility Tensor of LanthanideContaining Polymethylated-DOTA Complexes. J. Biomol. NMR 2016, 66 (2), 125-139.

(62) Ullrich, S. J.; Hölper, S.; Glaubitz, C. Paramagnetic Doping of a 7TM Membrane Protein in Lipid Bilayers by $\mathrm{Gd}^{3+}$-Complexes for Solid-State NMR Spectroscopy. J. Biomol. NMR 2014, $58(1), 27-35$.

(63) Göbl, C.; Resch, M.; Strickland, M.; Hartlmüller, C.; Viertler, M.; Tjandra, N.; Madl, T. Increasing the Chemical-Shift Dispersion of Unstructured Proteins with a Covalent Lanthanide Shift Reagent. Angew. Chemie - Int. Ed. 2016, 55 (47), 14847-14851.

(64) Graham, B.; Loh, C. T.; Swarbrick, J. D.; Ung, P.; Shin, J.; Yagi, H.; Jia, X.; Chhabra, S.; Barlow, N.; Pintacuda, G.; et al. DOTA-Amide Lanthanide Tag for Reliable Generation of Pseudocontact Shifts in Protein NMR Spectra. Bioconjug. Chem. 2011, 22 (10), 2118-2125.

(65) Nitsche, C.; Otting, G. Chapter 2: Intrinsic and Extrinsic Paramagnetic Probes; New Dev. NMR, 2018; 42-84.

(66) Kooshapur, H.; Schwieters, C. D.; Tjandra, N. Conformational Ensemble of Disordered Proteins Probed by Solvent Paramagnetic Relaxation Enhancement (SPRE). Angew. Chemie - 
Int. Ed. 2018, 57 (41), 13519-13522.

(67) Strickland, M.; Catazaro, J.; Rajasekaran, R.; Strub, M. P.; O’Hern, C.; Bermejo, G. A.; Summers, M. F.; Marchant, J.; Tjandra, N. Long-Range RNA Structural Information via a Paramagnetically Tagged Reporter Protein. J. Am. Chem. Soc. 2019, 141 (4), 1430-1434.

(68) Clore, G. M.; Iwahara, J. Theory, Practice, and Applications of Paramagnetic Relaxation Enhancement for the Characterization of Transient Low-Population States of Biological Macromolecules and Their Complexes. Chem. Rev. 2009, 109, 4108-4139.

(69) Li, W.; Zhang, Q.; Joos, J. J.; Smet, P. F.; Schmedt auf der Günne, J. Blind Spheres of Paramagnetic Dopants in Solid State NMR. Phys. Chem. Chem. Phys. 2019, 21, 10185-10194.

(70) Sengupta, I.; Nadaud, P. S.; Jaroniec, C. P. Protein Structure Determination with Paramagnetic Solid-State NMR Spectroscopy. Acc. Chem. Res. 2013, 46 (9), 2117-2126.

(71) Nadaud, P. S.; Helmus, J. J.; Sengupta, I.; Jaroniec, C. P. Rapid Acquisition of Multidimensional Solid-State NMR Spectra of Proteins Facilitated by Covalently Bound Paramagnetic Tags. J. Am. Chem. Soc. 2010, 132 (28), 9561-9563.

(72) Su, X. C.; Huber, T.; Dixon, N. E.; Otting, G. Site-Specific Labelling of Proteins with a Rigid Lanthanide-Binding Tag. ChemBioChem 2006, 7 (10), 1599-1604.

(73) Veglia, G.; Opella, S. J. Lanthanide Ion Binding to Adventitious Sites Aligns Membrane Proteins in Micelles for Solution NMR Spectroscopy. J. Am. Chem. Soc. 2000, 122, $11733-$ 11734.

(74) Olivieri, C.; Subrahmanian, M. V.; Xia, Y.; Kim, J.; Porcelli, F.; Veglia, G. Simultaneous Detection of Intra-and Inter-Molecular Paramagnetic Relaxation Enhancements in Protein Complexes. J. Biomol. NMR 2018, 70, 133-140.

(75) Iwahara, J.; Tang, C.; Marius Clore, G. Practical Aspects of ${ }^{1} \mathrm{H}$ Transverse Paramagnetic Relaxation Enhancement Measurements on Macromolecules. J. Magn. Reson. 2007, 184 (2), $185-195$.

(76) Yamamoto, K.; Xu, J.; Kawulka, K. E.; Vederas, J. C.; Ramamoorthy, A. Use of a CopperChelated Lipid Speeds up NMR Measurements from Membrane Proteins. J. Am. Chem. Soc. 2010, 132 (20), 6929-6931.

(77) Bernini, A.; Venditti, V.; Spiga, O.; Niccolai, N. Probing Protein Surface Accessibility with Solvent and Paramagnetic Molecules. Prog. Nucl. Magn. Reson. Spectrosc. 2009, 54 (3-4), 
$278-289$.

(78) Su, Y.; Mani, R.; Hong, M. Asymmetric Insertion of Membrane Proteins in Lipid Bilayers by Solid-State NMR Paramagnetic Relaxation Enhancement: A Cell-Penetrating Peptide Example. J. Am. Chem. Soc. 2008, 130 (27), 8856-8864.

(79) Sciacca, M. F. M.; Milardi, D.; Messina, G. M. L.; Marletta, G.; Brender, J. R.; Ramamoorthy, A.; La Rosa, C. Cations as Switches of Amyloid-Mediated Membrane Disruption Mechanisms: Calcium and IAPP. Biophys. J. 2013, 104 (1), 173-184.

(80) Hocking, H. G.; Zangger, K.; Madl, T. Studying the Structure and Dynamics of Biomolecules by Using Soluble Paramagnetic Probes. ChemPhysChem 2013, 14 (13), 3082-3094.

(81) Madl, T.; Bermel, W.; Zangger, K. Use of Relaxation Enhancements in a Paramagnetic Environment for the Structure Determination of Proteins Using NMR Spectroscopy. Angew. Chemie - Int. Ed. 2009, 48 (44), 8259-8262.

(82) Sengupta, I.; Nadaud, P. S.; Helmus, J. J.; Schwieters, C. D.; Jaroniec, C. P. Protein Fold Determined by Paramagnetic Magic-Angle Spinning Solid-State NMR Spectroscopy. Nat. Chem. 2012, 4 (5), 410-417.

(83) Bertini, I.; Bhaumik, A.; De Paëpe, G.; Griffin, R. G.; Lelli, M.; Lewandowski, J. R.; Luchinat, C. High-Resolution Solid-State NMR Structure of a 17.6 KDa Protein. J. Am. Chem. Soc. 2010, $132(3), 1032-1040$.

(84) Balayssac, S.; Bertini, I.; Lelli, M.; Luchinat, C.; Maletta, M. Paramagnetic Ions Provide Structural Restraints in Solid-State NMR of Proteins. J. Am. Chem. Soc. 2007, 129 (8), 2218 2219.

(85) Nadaud, P. S.; Helmus, J. J.; Höfer, N.; Jaroniec, C. P. Long-Range Structural Restraints in Spin-Labeled Proteins Probed by Solid-State Nuclear Magnetic Resonance Spectroscopy. $J$. Am. Chem. Soc. 2007, 129 (24), 7502-7503.

(86) Mandala, V. S.; Hong, M. High-Sensitivity Protein Solid-State NMR Spectroscopy. Curr. Opin. Struct. Biol. 2019, 58, 183-190.

(87) Bertmer, M. Paramagnetic Solid-State NMR of Materials. Solid State Nucl. Magn. Reson. 2017, 81, 1-7.

(88) Kolodziejski, W.; Klinowski, J. Kinetics of Cross-Polarization in Solid-State NMR: A Guide for Chemists. Chem. Rev. 2002, 102 (3), 613-628. 
(89) Inagaki, S.; Kawamura, I.; Sasaki, Y.; Yoshida, K.; Kubota, Y.; Naito, A. Drastic Sensitivity Enhancement in 29Si MAS NMR of Zeolites and Mesoporous Silica Materials by Paramagnetic Doping of $\mathrm{Cu}^{2+}$. Phys. Chem. Chem. Phys. 2013, 15 (32), 13523-13531.

(90) Pines, A.; Gibby, M. G.; Waugh, J. S. Proton-Enhanced NMR of Dilute Spins in Solids. J. Chem. Phys. 1973, 569, 569-590.

(91) Schaefer, J.; Stejskal, E. O. Carbon-13 Nuclear Magnetic Resonance of Polymers Spinning at the Magic Angle. J. Am. Chem. Soc. 1976, 98, 1031-1032.

(92) Schaefer, J.; Stejskal, E. O.; Buchdahl, R. Magic-Angle 13C NMR Analysis of Motion in Solid Glassy Polymers. Macromolecules 1977, 10, 384-405.

(93) Wickramasinghe, N. P.; Kotecha, M.; Samoson, A.; Past, J.; Ishii, Y. Sensitivity Enhancement in 13C Solid-State NMR of Protein Microcrystals by Use of Paramagnetic Metal Ions for Optimizing 1H T1 Relaxation. J. Magn. Reson. 2007, 184, 350-356.

(94) Ganapathy S.; Naito A.; McDowell C.A. Paramagnetic Doping as an Aid in Obtaining HighResolution 13C NMR Spectra of Biomolecules in the Solid State. J. Am. Chem. Soc. 1981, 103, 6011-6015.

(95) Linser, R.; Chevelkov, V.; Diehl, A.; Reif, B. Sensitivity Enhancement Using Paramagnetic Relaxation in MAS Solid-State NMR of Perdeuterated Proteins. J. Magn. Reson. 2007, 189 (2), 209-216.

(96) Wickramasinghe, N. P.; Parthasarathy, S.; Jones, C. R.; Bhardwaj, C.; Long, F.; Kotecha, M.; Mehboob, S.; Fung, L. W. M.; Past, J.; Samoson, A.; et al. Nanomole-Scale Protein Solid-State NMR by Breaking Intrinsic 1 H T1 Boundaries. Nat. Methods 2009, 6 (3), 215-218.

(97) Ishii, Y.; Wickramasinghe, A.; Matsuda, I.; Endo, Y.; Ishii, Y.; Nishiyama, Y.; Nemoto, T.; Kamihara, T. Progress in Proton-Detected Solid-State NMR (SSNMR): Super-Fast 2D SSNMR Collection for Nano-Mole-Scale Proteins. J. Magn. Reson. 2018, 286, 99-109.

(98) Lutker, K. M.; Quiñones, R.; Xu, J.; Ramamoorthy, A.; Matzger, A. J. Polymorphs and Hydrates of Acyclovir. J. Pharm. Sci. 2011, 100 (3), 949-963.

(99) Mroue, K. H.; Nishiyama, Y.; Kumar Pandey, M.; Gong, B.; McNerny, E.; Kohn, D. H.; Morris, M. D.; Ramamoorthy, A. Proton-Detected Solid-State NMR Spectroscopy of Bone with Ultrafast Magic Angle Spinning. Sci. Rep. 2015, 5, 1-10.

(100) Mroue, K. H.; MacKinnon, N.; Xu, J.; Zhu, P.; McNerny, E.; Kohn, D. H.; Morris, M. D.; 
Ramamoorthy, A. High-Resolution Structural Insights into Bone: A Solid-State NMR Relaxation Study Utilizing Paramagnetic Doping. J. Phys. Chem. B 2012, 116 (38), 1165611661.

(101) Zhou, L.; Li, S.; Su, Y.; Li, B.; Deng, F. Paramagnetic Relaxation Enhancement Solid-State NMR Studies of Heterogeneous Catalytic Reaction over HY Zeolite Using Natural Abundance Reactant. Solid State Nucl. Magn. Reson. 2015, 66, 29-32.

(102) Gorkov, P. L.; Chekmenev, E. Y.; Li, C.; Cotten, M.; Buffy, J. J.; Traaseth, N. J.; Veglia, G.; Brey, W. W. Using Low-E Resonators to Reduce RF Heating in Biological Samples for Static Solid-State NMR up to $900 \mathrm{MHz}$. J. Magn. Reson. 2007, 185 (1), 77-93.

(103) Wang, J.; Zhang, Z.; Zhao, W.; Wang, L.; Yang, J. Heating and Temperature Gradients of Lipid Bilayer Samples Induced by RF Irradiation in MAS Solid-State NMR Experiments. Magn. Reson. Chem. 2016, 54 (9), 753-759.

(104) D’Espinose De Lacaillerie, J. B.; Jarry, B.; Pascui, O.; Reichert, D. "cooking the Sample": Radiofrequency Induced Heating during Solid-State NMR Experiments. Solid State Nucl. Magn. Reson. 2005, 28 (2-4), 225-232.

(105) Yamamoto, K.; Ermakov, V. L.; Lee, D. K.; Ramamoorthy, A. PITANSEMA-MAS, a SolidState NMR Method to Measure Heteronuclear Dipolar Couplings under MAS. Chem. Phys. Lett. 2005, 408 (1-3), 118-122.

(106) Stringer, J. A.; Bronnimann, C. E.; Mullen, C. G.; Zhou, D. H.; Stellfox, S. A.; Li, Y.; Williams, E. H.; Rienstra, C. M. Reduction of RF-Induced Sample Heating with a Scroll Coil Resonator Structure for Solid-State NMR Probes. J. Magn. Reson. 2005, 173 (1), 40-48.

(107) Demers, J. P.; Vijayan, V.; Becker, S.; Lange, A. Tailored Low-Power Cross-Polarization under Fast Magic-Angle Spinning. J. Magn. Reson. 2010, 205 (2), 216-223.

(108) Wickramasinghe, N. P.; Shaibat, M. A.; Jones, C. R.; Casabianca, L. B.; De Dios, A. C.; Harwood, J. S.; Ishii, Y. Progress in 13C and 1H Solid-State Nuclear Magnetic Resonance for Paramagnetic Systems under Very Fast Magic Angle Spinning. J. Chem. Phys. 2008, 128 (5), 052210.

(109) Ishii, Y.; Yesinowski, J. P.; Tycko, R. Sensitivity Enhancement in Solid-State 13C NMR of Synthetic Polymers and Biopolymers by 1H NMR Detection with High-Speed Magic Angle Spinning. J. Am. Chem. Soc. 2001, 123, 2921-2922. 
(110) Shekar, S. C.; Lee, D. K.; Ramamoorthy, A. An Experimental Strategy to Dramatically Reduce the RF Power Used in Cross Polarization Solid-State NMR Spectroscopy [23]. J. Am. Chem. Soc. 2001, 123 (30), 7467-7468.

(111) Li, C.; Mo, Y.; Hu, J.; Chekmenev, E.; Tian, C.; Gao, F. P.; Fu, R.; Gor'kov, P.; Brey, W.; Cross, T. A. Analysis of RF Heating and Sample Stability in Aligned Static Solid-State NMR Spectroscopy. J. Magn. Reson. 2006, 180 (1), 51-57.

(112) Cournia, Z.; Allen, T. W.; Andricioaei, I.; Antonny, B.; Baum, D.; Brannigan, G.; Buchete, N.-V.; Deckman, J. T.; Delemotte, L.; del Val, C.; et al. Membrane Protein Structure, Function, and Dynamics: A Perspective from Experiments and Theory. J. Membr. Biol. 2015, 248 (4), $611-640$.

(113) Fry, M. Y.; Clemons, W. M. J. Complexity in Targeting Membrane Proteins. Science. 2018, 359 (6374), 390-391.

(114) Misiewicz, J.; Afonin, S.; Grage, S. L.; Van Den Berg, J.; Strandberg, E.; Wadhwani, P.; Ulrich, A. S. Action of the Multifunctional Peptide BP100 on Native Biomembranes Examined by Solid-State NMR. J. Biomol. NMR 2015, 61, 287-298.

(115) Wiegand, T.; Cadalbert, R.; Lacabanne, D.; Böckmann, A.; Meier, B. H.; Timmins, J.; Terradot, L. The Conformational Changes Coupling ATP Hydrolysis and Translocation in a Bacterial. Nat. Commun. 2019, 10 (31), 1-11.

(116) Muñoz-García, J. C.; Inacio dos Reis, R.; Taylor, R. J.; Henry, A. J.; Watts, A. NanodiscTargeted STD NMR Spectroscopy Reveals Atomic Details of Ligand Binding to Lipid Environments. ChemBioChem 2018, 19, 1022-1025.

(117) Pinto, C.; Mance, D.; Sinnige, T.; Daniëls, M.; Weingarth, M.; Baldus, M. Formation of the $\beta$ Barrel Assembly Machinery Complex in Lipid Bilayers as Seen by Solid-State NMR. Nat. Commun. 2018, 9, 4135.

(118) Pinto, C.; Mance, D.; Julien, M.; Daniels, M.; Weingarth, M.; Baldus, M. Studying Assembly of the BAM Complex in Native Membranes by Cellular Solid-State NMR Spectroscopy. $J$. Struct. Biol. 2019, 206, 1-11.

(119) Liebi, M.; Kuster, S.; Kohlbrecher, J.; Ishikawa, T.; Fischer, P.; Walde, P.; Windhab, E. J. Cholesterol-Diethylenetriaminepentaacetate Complexed with Thulium Ions Integrated into Bicelles to Increase Their Magnetic Alignability. J. Phys. Chem. B 2013, 117, 14743-14748. 
(120) Isabettini, S.; Liebi, M.; Kohlbrecher, J.; Ishikawa, T.; Fischer, P.; Windhab, E. J.; Walde, P.; Kuster, S. Mastering the Magnetic Susceptibility of Magnetically Responsive Bicelles with 3ß-Amino-5-Cholestene and Complexed Lanthanide Ions. Phys. Chem. Chem. Phys. 2017, 19, $10820-10824$.

(121) Frey, L.; Lakomek, N. A.; Riek, R.; Bibow, S. Micelles, Bicelles, and Nanodiscs: Comparing the Impact of Membrane Mimetics on Membrane Protein Backbone Dynamics. Angew. Chemie - Int. Ed. 2017, 56, 380-383.

(122) Routledge, S. J.; Linney, J. A.; Goddard, A. D. Liposomes as Models for Membrane Integrity. Biochem. Soc. Trans. 2019, 1-14.

(123) Dürr, U. H. N.; Soong, R.; Ramamoorthy, A. When Detergent Meets Bilayer: Birth and Coming of Age of Lipid Bicelles. Prog. Nucl. Magn. Reson. Spectrosc. 2013, 69, 1-22.

(124) Sanders, C. R.; Prosser, R. S. Bicelles: A Model Membrane System for All Seasons? Structure 1998, 6 (10), 1227-1234.

(125) Sanders, C. R.; Hare, B. J.; Howard, K. P.; Prestegard, J. H. Magnetically-Oriented Phospholipid Micelles as a Tool for the Study of Membrane-Associated Molecules. Progress in Nuclear Magnetic Resonance Spectroscopy. Pergamon, 1994, 421-444.

(126) Baumgartner, M. E.; Reckey, P. Q.; Kohlbrecher, J.; Ishikawa, T.; Fischer, P.; Windhab, E. J.; Kuster, S. Methods for Generating Highly Magnetically Responsive Lanthanide- Chelating Phospholipid Polymolecular Assemblies. Langmuir 2017, 33, 6363-6371.

(127) Isabettini, S.; Liebi, M.; Kohlbrecher, J.; Ishikawa, T.; Windhab, E. J.; Fischer, P.; Walde, P.; Kuster, S. Tailoring Bicelle Morphology and Thermal Stability with Lanthanide-Chelating Cholesterol Conjugates. Langmuir 2016, 32, 9005-9014.

(128) Isabettini, S.; Stucki, S.; Massabni, S.; Baumgartner, M. E.; Reckey, P. Q.; Kohlbrecher, J.; Ishikawa, T.; Windhab, E. J.; Fischer, P.; Kuster, S. Development of Smart Optical Gels with Highly Magnetically Responsive Bicelles. ACS Appl. Mater. Interfaces 2018, 10, 8926-8936.

(129) Isabettini, S.; Massabni, S.; Hodzic, A.; Durovic, D.; Kohlbrecher, J.; Ishikawa, T.; Fischer, P.; Windhab, E. J.; Walde, P.; Kuster, S. Molecular Engineering of Lanthanide Ion Chelating Phospholipids Generating Assemblies with a Switched Magnetic Susceptibility. Phys. Chem. Chem. Phys. 2017, 19, 20991-21002.

(130) Isabettini, S.; Massabni, S.; Kohlbrecher, J.; Schuler, L. D.; Walde, P.; Sturm, M.; Windhab, 
E. J.; Fischer, P.; Kuster, S. Understanding the Enhanced Magnetic Response of Aminocholesterol Doped Lanthanide-Ion-Chelating Phospholipid Bicelles. Langmuir 2017, $33,8533-8544$.

(131) Bayburt, T. H.; Grinkova, Y. V; Sligar, S. G. Self-Assembly of Discoidal Phospholipid Bilayer Nanoparticles with Membrane Scaffold Proteins. Nano Lett. 2002, 2 (8), 853-856.

(132) Knowles, T. J.; Finka, R.; Smith, C.; Lin, Y.-P.; Dafforn, T.; Overduin, M. Membrane Proteins Solubilized Intact in Lipid Containing Nanoparticles Bounded by Styrene Maleic Acid Copolymer. J. Am. Chem. Soc. 2009, 131 (22), 7484-7485.

(133) Denisov, I. G.; Sligar, S. G. Nanodiscs in Membrane Biochemistry and Biophysics. Chemical Reviews. 2017, 4669-4713.

(134) Oluwole, A. O.; Danielczak, B.; Meister, A.; Babalola, J. O.; Vargas, C.; Keller, S. Solubilization of Membrane Proteins into Functional Lipid-Bilayer Nanodiscs Using a Diisobutylene/Maleic Acid Copolymer. Angew. Chemie - Int. Ed. 2017, 56 (7), 1919-1924.

(135) Yasuhara, K.; Arakida, J.; Ravula, T.; Ramadugu, S. K.; Sahoo, B.; Kikuchi, J.-I.; Ramamoorthy, A. Spontaneous Lipid Nanodisc Fomation by Amphiphilic Polymethacrylate Copolymers. J Am Chem Soc 2017, 139, 18657-18663.

(136) Hardin, N. Z.; Ravula, T.; Mauro, G. Di; Ramamoorthy, A. Hydrophobic Functionalization of Polyacrylic Acid as a Versatile Platform for the Development of Polymer Lipid Nanodisks. Small 2019, 15 (9).

(137) Ravula, T.; Hardin, N. Z.; Ramadugu, S. K.; Cox, S. J.; Ramamoorthy, A. Formation of pHResistant Monodispersed Polymer-Lipid Nanodiscs. Angew. Chemie Int. Ed. 2018, 57 (5), $1342-1345$.

(138) Ravula, T.; Hardin, N. Z.; Ramadugu, S. K.; Ramamoorthy, A. pH Tunable and Divalent Metal Ion Tolerant Polymer Lipid Nanodiscs. Langmuir 2017, 33 (40), 10655-10662.

(139) Ravula, T.; Ramadugu, S. K.; Di Mauro, G.; Ramamoorthy, A. Bioinspired, Size-Tunable SelfAssembly of Polymer-Lipid Bilayer Nanodiscs. Angew. Chemie - Int. Ed. 2017, 56 (38), $11466-11470$.

(140) Lindhoud, S.; Carvalho, V.; Pronk, J. W.; Aubin-Tam, M. E. SMA-SH: Modified StyreneMaleic Acid Copolymer for Functionalization of Lipid Nanodiscs. Biomacromolecules 2016, $17(4), 1516-1522$. 
(141) Fiori, M. C.; Jiang, Y.; Altenberg, G. A.; Liang, H. Polymer-Encased Nanodiscs with Improved Buffer Compatibility. Sci. Rep. 2017, 7, 7-10.

(142) Hall, S. C. L.; Tognoloni, C.; Charlton, J.; Bragginton, É. C.; Rothnie, A. J.; Sridhar, P.; Wheatley, M.; Knowles, T. J.; Arnold, T.; Edler, K. J.; et al. An Acid-Compatible Co-Polymer for the Solubilization of Membranes and Proteins into Lipid Bilayer-Containing Nanoparticles. Nanoscale 2018, 10, 10609-10619.

(143) Radoicic, J.; Park, S. H.; Opella, S. J. Macrodiscs Comprising SMALPs for Oriented Sample Solid-State NMR Spectroscopy of Membrane Proteins. Biophys. J. 2018, 115 (1), 22-25.

(144) Brown, L. S.; Ladizhansky, V. Membrane Proteins in Their Native Habitat as Seen by SolidState NMR Spectroscopy. Protein Sci. 2015, 24 (9), 1333-1346.

(145) Purusottam, R. N.; Sénicourt, L.; Lacapère, J. J.; Tekely, P. Probing the Gel to LiquidCrystalline Phase Transition and Relevant Conformation Changes in Liposomes by ${ }^{13} \mathrm{C}$ MagicAngle Spinning NMR Spectroscopy. Biochim. Biophys. Acta - Biomembr. 2015, 1848 (12), 3134-3139.

(146) Opella, S. J. Structure Determination of Membrane Proteins in Their Native Phospholipid Bilayer Environment by Rotationally Aligned Solid-State NMR Spectroscopy. Acc. Chem. Res. 2013, 46 (9), 2145-2153.

(147) Lam, D.; Zhuang, J.; Cohen, L. S.; Arshava, B.; Naider, F. R.; Tang, M. Effects of Chelator Lipids, Paramagnetic Metal Ions and Trehalose on Liposomes by Solid-State NMR. Solid State Nucl. Magn. Reson. 2018, 94, 1-6.

(148) Yamamoto, K.; Pearcy, P.; Ramamoorthy, A. Bicelles Exhibiting Magnetic Alignment for a Broader Range of Temperatures: A Solid-State NMR Study. Langmuir 2014, 30 (6), 16221629.

(149) Liebi, M.; Kuster, S.; Kohlbrecher, J.; Ishikawa, T.; Fischer, P.; Walde, P.; Windhab, E. J. Magnetically Enhanced Bicelles Delivering Switchable Anisotropy in Optical Gels. ACS Appl. Mater. Interfaces 2014, 6, 1100-1105.

(150) Liebi, M.; Van Rhee, P. G.; Christianen, P. C. M.; Kohlbrecher, J.; Fischer, P.; Walde, P.; Windhab, E. J. Alignment of Bicelles Studied with High-Field Magnetic Birefringence and Small-Angle Neutron Scattering Measurements. Langmuir 2013, 29, 3467-3473.

(151) Wang, S.; Gopinath, T.; Veglia, G. Application of Paramagnetic Relaxation Enhancements to 
Accelerate the Acquisition of 2D and 3D Solid-State NMR Spectra of Oriented Membrane Proteins. Methods 2018, 138-139, 54-61.

(152) Nevzorov, A. A.; Opella, S. J. A "Magic Sandwich" Pulse Sequence with Reduced Offset Dependence for High-Resolution Separated Local Field Spectroscopy. J. Magn. Reson. 2003, 164 (1), 182-186.

(153) Ramamoorthy, A.; Wu, C. H.; Opella, S. J. Three-Dimensional Solid-State NMR Experiment That Correlates the Chemical Shift and Dipolar Coupling Frequencies of Two Heteronuclei. $J$. Magn. Reson. B 1995, 107 (1), 88-90.

(154) Yamamoto, K.; Lee, D. K.; Ramamoorthy, A. Broadband-PISEMA Solid-State NMR Spectroscopy. Chem. Phys. Lett. 2005, 407, 289-293.

(155) Ramamoorthy, A.; Wei, Y.; Lee, D. K. PISEMA Solid-State NMR Spectroscopy. In Annual Reports on NMR Spectroscopy; 2004; Vol. 52, 1-52.

(156) Wu, C. H.; Ramamoorthy, A.; Opella, S. J. High-Resolution Heteronuclear Dipolar Solid-State NMR Spectroscopy. J. Magn. Reson. Ser. A 1994, 109, 270-272.

(157) Prosser, R. S.; Bryant, H.; Bryant, R. G.; Vold, R. R. Lanthanide Chelates as Bilayer Alignment Tools in NMR Studies of Membrane-Associated Peptides. J. Magn. Reson. 1999, 141 (2), 256260.

(158) Howard, K. P.; Opella, S. J. High-Resolution Solid-State NMR Spectra of Integral Membrane Proteins Reconstituted into Magnetically Oriented Phospholipid Bilayers. J. Magn. Reson. Ser. $B$ 1996, 112, 91-94.

(159) Bali, A. P.; Sahu, I. D.; Craig, A. F.; Clark, E. E.; Burridge, K. M.; Dolan, M. T.; DabneySmith, C.; Konkolewicz, D.; Lorigan, G. A. Structural Characterization of Styrene-Maleic Acid Copolymer-Lipid Nanoparticles (SMALPs) Using EPR Spectroscopy. Chem. Phys. Lipids 2019, 220, 6-13.

(160) Ravula, T.; Ramamoorthy, A. Magnetic-Alignment of Polymer Macro-Nanodiscs Enable Residual Dipo- Lar Couplings Based High-Resolution Structural Studies by NMR. bioRxiv 2019, 1-5.

(161) Chakrabarty, T.; Goldin, N.; Feintuch, A.; Houben, L.; Leskes, M. Paramagnetic Metal-Ion Dopants as Polarization Agents for Dynamic Nuclear Polarization NMR Spectroscopy in Inorganic Solids. ChemPhysChem 2018, 19 (17), 2139-2142. 
(162) Zagdoun, A.; Casano, G.; Ouari, O.; Schwarzwälder, M.; Rossini, A. J.; Aussenac, F.; Yulikov, M.; Jeschke, G.; Copéret, C.; Lesage, A.; et al. Large Molecular Weight Nitroxide Biradicals Providing Efficient Dynamic Nuclear Polarization at Temperatures up to $200 \mathrm{~K}$. J. Am. Chem. Soc. 2013, 135 (34), 12790-12797.

(163) Zagdoun, A.; Casano, G.; Ouari, O.; Lapadula, G.; Rossini, A. J.; Lelli, M.; Baffert, M.; Gajan, D.; Veyre, L.; Maas, W. E.; et al. A Slowly Relaxing Rigid Biradical for Efficient Dynamic Nuclear Polarization Surface-Enhanced NMR Spectroscopy: Expeditious Characterization of Functional Group Manipulation in Hybrid Materials. J. Am. Chem. Soc. 2012, 134 (4), 2284 2291.

(164) Sauvée, C.; Casano, G.; Abel, S.; Rockenbauer, A.; Akhmetzyanov, D.; Karoui, H.; Siri, D.; Aussenac, F.; Maas, W.; Weber, R. T.; et al. Tailoring of Polarizing Agents in the BTurea Series for Cross-Effect Dynamic Nuclear Polarization in Aqueous Media. Chem. - A Eur. J. 2016, 22 (16), 5598-5606.

(165) Song, C.; Hu, K.-N.; Joo, C.-G.; Swager, T. M.; Griffin, R. G. TOTAPOL: A Biradical Polarizing Agent for Dynamic Nuclear Polarization Experiments in Aqueous Media. 2006.

(166) Hall, D. A.; Maus, D. C.; Gerfen, G. J.; Inati, S. J.; Becerra, L. R.; Dahlquist, F. W.; Griffin, R. G. Polarization-Enhanced NMR Spectroscopy of Biomolecules in Frozen Solution. Science (80-. ). 1997, 276 (5314), 930-932.

(167) Gerfen, G. J.; Becerra, L. R.; Hall, D. A.; Griffin, R. G.; Temkin, R. J.; Singel, D. J. High Frequency $(140 \mathrm{GHz})$ Dynamic Nuclear Polarization: Polarization Transfer to a Solute in Frozen Aqueous Solution. J. Chem. Phys. 1995, 102 (24), 9494-9497.

(168) Thurber, K. R.; Tycko, R. Biomolecular Solid State NMR with Magic-Angle Spinning at 25 K. J. Magn. Reson. 2008, 195 (2), 179-186.

(169) Jaudzems, K.; Polenova, T.; Pintacuda, G.; Oschkinat, H.; Lesage, A. DNP NMR of Biomolecular Assemblies. J. Struct. Biol. 2019, 206 (1), 90-98.

(170) Rogawski, R.; Sergeyev, I. V; Zhang, Y.; Tran, T. H.; Li, Y.; Tong, L.; Mcdermott, A. E. NMR Signal Quenching from Bound Biradical Affinity Reagents in DNP Samples. J. Phys. Chem. B 2017, 121, 10770-10781.

(171) Salnikov, E. S.; Abel, S. Ø.; Karthikeyan, G.; Karoui, H.; Aussenac, F.; Tordo, P.; Bechinger, B.; Ouari, O. Dynamic Nuclear Polarization/Solid-State NMR Spectroscopy of Membrane Polypeptides: Free-Radical Optimization for Matrix-Free Lipid Bilayer Samples. 
ChemPhysChem 2017, 18, 2103-2113.

(172) Gordon, J. W.; Fain, S. B.; Rowland, I. J. Effect of Lanthanide Ions on Dynamic Nuclear Polarization Enhancement and Liquid-State $\mathrm{T}_{1}$ Relaxation. Magn. Reson. Med. 2012, 68 (6), 1949-1954.

(173) Lilly Thankamony, A. S.; Wittmann, J. J.; Kaushik, M.; Corzilius, B. Dynamic Nuclear Polarization for Sensitivity Enhancement in Modern Solid-State NMR. Progress in Nuclear Magnetic Resonance Spectroscopy. 2017, 120-195.

(174) Kubicki, D. J.; Casano, G.; Schwarzwälder, M.; Abel, S.; Sauvée, C.; Ganesan, K.; Yulikov, M.; Rossini, A. J.; Jeschke, G.; Copéret, C.; et al. Rational Design of Dinitroxide Biradicals for Efficient Cross-Effect Dynamic Nuclear Polarization. Chem. Sci. 2016, 7 (1), 550-558.

(175) Niedbalski, P.; Parish, C.; Wang, Q.; Hayati, Z.; Song, L.; Martins, A. F.; Sherry, A. D.; Lumata, L. Transition Metal Doping Reveals Link between Electron $\mathrm{T}_{1}$ Reduction and ${ }^{13} \mathrm{C}$ Dynamic Nuclear Polarization Efficiency. J. Phys. Chem. A 2017, 121 (48), 9221-9228.

(176) Salnikov, E. S.; Aussenac, F.; Abel, S.; Purea, A.; Tordo, P.; Ouari, O.; Bechinger, B. Dynamic Nuclear Polarization / Solid-State NMR of Membranes. Thermal Effects and Sample Geometry. Solid State Nucl. Magn. Reson. 2019, 100, 70-76.

(177) Niedbalski, P.; Parish, C.; Wang, Q.; Kiswandhi, A.; Hayati, Z.; Song, L.; Lumata, L. 13C Dynamic Nuclear Polarization Using a Trimeric $\mathrm{Gd}^{3+}$ Complex as an Additive. J. Phys. Chem. A 2017, 121 (27), 5127-5135.

(178) Thurber, K. R.; Tycko, R. Perturbation of Nuclear Spin Polarizations in Solid State NMR of Nitroxide-Doped Samples by Magic-Angle Spinning without Microwaves. J. Chem. Phys. 2014, 140, 184201.

(179) Niedbalski, P.; Parish, C.; Kiswandhi, A.; Fidelino, L.; Khemtong, C.; Hayati, Z.; Song, L.; Martins, A.; Sherry, A. D.; Lumata, L. Influence of $\mathrm{Dy}^{3+}$ and $\mathrm{Tb}^{3+}$ Doping on ${ }^{13} \mathrm{C}$ Dynamic Nuclear Polarization. J. Chem. Phys. 2017, 146 (1), 14303.

(180) Kaushik, M.; Bahrenberg, T.; Can, T. V; Caporini, M. A.; Silvers, R.; Heiliger, J.; Smith, A. A.; Schwalbe, H.; Griffin, R. G.; Corzilius, B. Gd(III) and Mn(II) Complexes for Dynamic Nuclear Polarization: Small Molecular Chelate Polarizing Agents and Applications with SiteDirected Spin Labeling of Proteins. Phys. Chem. Chem. Phys 2016, 18, 27205-27218.

(181) Corzilius, B.; Smith, A. A.; Barnes, A. B.; Luchinat, C.; Bertini, I.; Griffin, R. G. High-Field 
Dynamic Nuclear Polarization with High-Spin Transition Metal Ions. J. Am. Chem. Soc. 2011, 133 (15), 5648-5651.

(182) Corzilius, B.; Michaelis, V. K.; Penzel, S. A.; Ravera, E.; Smith, A. A.; Luchinat, C.; Griffin, R. G. Dynamic Nuclear Polarization of ${ }^{1} \mathrm{H},{ }^{13} \mathrm{C}$, and ${ }^{59} \mathrm{Co}$ in a Tris(Ethylenediamine)Cobalt(III) Crystalline Lattice Doped with Cr(III). J. Am. Chem. Soc. 2014, 136, 11716-11727. 


\section{Chapter 3}

Lipid-Nanodiscs Formed by Paramagnetic Metal Chelated Polymer for Fast NMR Data Acquisition

The content for this chapter is included in the following research article:

Di Mauro Giacomo M., Hardin Nathaniel Z., Ramamoorthy Ayyalusamy, Lipid-nanodiscs formed by paramagnetic metal chelated polymer for fast NMR data acquisition, BBA Biomembranes, 1862, 9, 09/2020, 183332 


\subsection{Introduction}

Membrane proteins are ubiquitous in every cell and perform a plethora of fundamental functions for the living organisms ${ }^{1}$. Despite their importance, there is still a large gap in the structural studies of membrane proteins when compared to their soluble protein counterparts. This is mainly because of the difficulties in mimicking native lipid membranes and using the membrane mimetics to stabilize native-like folding and function of membrane proteins ${ }^{2}$. While detergents are continued to be used in the purification and structural studies of membrane proteins, it is known that detergents are capable of denaturing membrane proteins. Other well-utilized model membranes are bicelles and liposomes, which also have limitations ${ }^{3}$. Lipid nanodiscs, in recent years, have shown great potential in the structural biology of membrane proteins. ${ }^{1,4-10}$. Nanodiscs are disc-shaped lipid bilayer patches surrounded by an amphiphilic macromolecule that can be made up of membrane scaffold proteins (MSPs) ${ }^{1,11-13}$, peptides ${ }^{14}$, or synthetic-polymers ${ }^{5,9,15-21}$. Although all types of nanodiscs are increasingly used, most MSP based nanodiscs are limited by size flexibility. The background signal from both MSP based and peptide-based nanodiscs are not desirable in the characterization of reconstituted membrane proteins by several biophysical techniques. Most of these nanodiscs exhibit poor stability against acidic $\mathrm{pH}$ and the presence of divalent metal ions such as calcium and magnesium ions ${ }^{18}$. Overall, the synthetic polymer-based nanodiscs have been shown to provide a number of flexibilities and choices, enabling a variety of applications. Many different types of polymer belts have been reported in the literature. They have been used to demonstrate that polymerbased nanodisc technology is suitable for studying biomacromolecules by most biophysical techniques, including both solution and solid-state NMR spectroscopy ${ }^{15,22-27}$. While NMR is unique in rendering the measurement of atomic-resolution dynamics at different time scales, it has the significant disadvantage of being a relatively low-sensitivity technique ${ }^{28}$. This disadvantage is amplified by the instability or scarce amount of most biologically interesting membrane proteins and also to characterize short-lived intermediates such as amyloid oligomers. This low sensitivity is most commonly overcome by either expensive isotopic labeling and encumberingly long experimental times, which is not desirable for heat-sensitive proteins ${ }^{28-34}$. Another solution is to increase the sample's concentration, but it is often untenable for many protein systems due to the potential instability and aggregation issues of the proteins such as amyloid proteins. One of the most important ways to increase the sensitivity of NMR is by the addition of paramagnetic dopants to utilize the paramagnetic relaxation enhancement (PRE) $)^{35-41}$. 
PRE effect can be used to enhance NMR sensitivity by shortening the spin-lattice $\left(\mathrm{T}_{1}\right)$ relaxation properties of the nuclei ${ }^{42,43}$. Briefly, relaxation is a process in which the thermal equilibrium of the nuclear spin states is regained after the perturbation of applied radiofrequency (R.F.) pulses. The relaxation of multiple-spin systems can be an extremely complex process to describe, which can be further complicated by chemical exchange and local motions. However, it can be phenomenologically described by two of the major relaxation mechanisms: longitudinal (or spin-lattice or $\mathrm{T}_{1}$ ) and transverse (or spin-spin or $\mathrm{T}_{2}$ ) relaxations as defined by the Bloch equations ${ }^{44}$. PRE-NMR involves the introduction of a paramagnetic salt into the sample or external paramagnetic tags in the investigated system. Based on the chosen probe, the PRE effect can alter the relaxation in different ways $^{43,45-47}$. Unfortunately, it is not possible to separately influence $T_{1}$ and $T_{2}$ values; so, while affecting one of the relaxation parameters, it is inevitable to change the other. This aspect can cause undesired effects on the spectra, such as line-broadening (related to $\mathrm{T}_{2}$-shortening) and peak-shifts (due to hyperfine shift $)^{35,48-54}$ that can negatively affect the overall quality of the resultant spectra. Additionally, sample preparation is complicated by a further step of "tagging," which need not always proceed to completion ${ }^{48}$.

Since the PRE effect can be used to speed-up the $T_{1}$ process, it can be utilized to accelerate data acquisition by reducing the recycle delay between successive scans used for signal averaging in NMR experiments. In addition, the paramagnetic effect induced shift in resonance frequency (known as hyperfine shift and composed of two contributions: the contact and the pseudo-contact shifts or PCS) can be used to obtain structural information such as topological analysis and distance measurements since paramagnetic effects are typically proportional to $1 / \mathrm{r}^{\mathrm{n}}$ ( $\mathrm{n}=6$ for PRE and 3 for PCS), where $\mathrm{r}$ is the distance between the paramagnetic tag and the investigated nuclei ${ }^{35}$.

The versatility and flexibility of paramagnetic methods are benefited from a large pool of paramagnetic tags to choose from and the ways of incorporation to access the sites of interest ${ }^{55-62}$. Indeed, the use of diamagnetic and paramagnetic ions bound to specific positions or solvent-exposed surfaces of macromolecules or supramolecular aggregates both in solution and solid-state can reveal structural and topological information.

We recently reported a nanodisc forming synthetic polymer, SMA-EA-DOTA ${ }^{63}$. In addition to the already reported advantages of forming polymer-based lipid-nanodiscs, this copolymer enables the introduction of paramagnetic metal ions to the DOTA units in the polymer located on the outer rim polymer-nanodiscs. Thus, this DOTA-unit containing polymer avoids the presence of free paramagnetic ions in solution and the use of paramagnetically-tagged lipids ${ }^{43}$. As a result, an SMAEA-DOTA polymer-based nanodisc offers a planar lipid bilayer to reconstitute membrane protein(s) 
without interference from the added paramagnetic metal ions. The aim of this study is to reduce the spin-lattice relaxation time $\left(T_{1}\right)$ of the sample with a minimum line broadening due to a reduced spinspin relaxation time $\left(\mathrm{T}_{2}\right)$ by using the paramagnetic metals chelated to the DOTA units of the polymers in nanodiscs. Due to the size flexibility of polymer nanodiscs ${ }^{64}$, a wide variety of NMR conditions can be utilized for solution NMR, partial alignment for RDC measurements by solution NMR, and a higher degree of alignment for solid-state NMR experiments ${ }^{65}$. Because of this flexibility, a library of a variety of metal ions for differing conditions of PRE is needed. In addition, the introduction of paramagnetic metals can enable the applications of EPR experiments ${ }^{66,67}$. In this study, we report a systematic investigation of the PRE effects from five different paramagnetic metal ions $\left(\mathrm{Eu}^{3+}, \mathrm{Gd}^{3+}, \mathrm{Dy}^{3+}, \mathrm{Er}^{3+}, \mathrm{Yb}^{3+}\right)$ for NMR applications ${ }^{48,52,53,68-73}$.

\subsection{Materials and Methods}

\subsubsection{Reagents and Materials}

Poly(Styrene-co-Maleic Anhydride)-Cumene terminated, SMAnh, $\mathrm{M}_{\mathrm{w}} \sim 1.6 \mathrm{kDa}$, anhydrous 1Methyl2-pyrrolidone (NMP), 2-Aminoethanol (EA), Triethylamine, Trifluoroacetic Acid (TFA), Diethyl Ether $\left(\mathrm{Et}_{2} \mathrm{O}\right)$, Hydrochloric Acid $(\mathrm{HCl})$, Sodium Hydroxide $(\mathrm{NaOH})$, 4-(2Hydroxyethyl)Piperazine-1-ethanesulfonic acid (HEPES), Sodium Chloride (NaCl), Europium(III) Chloride Hexahydrate $\left(\mathrm{EuCl}_{3} \cdot 6 \mathrm{H}_{2} \mathrm{O}\right)$, Gadolinium(III) Chloride Hexahydrate $\left(\mathrm{GdCl}_{3} \cdot 6 \mathrm{H}_{2} \mathrm{O}\right)$, Dysprosium(III) Chloride Hexahydrate $\left(\mathrm{DyCl}_{3} \cdot 6 \mathrm{H}_{2} \mathrm{O}\right)$, Erbium(III) Chloride Hexahydrate $\left(\mathrm{ErCl}_{3} \cdot 6 \mathrm{H}_{2} \mathrm{O}\right)$, Ytterbium(III) Chloride Hexahydrate $\left(\mathrm{YbCl}_{3} \cdot 6 \mathrm{H}_{2} \mathrm{O}\right)$ were purchased from SigmaAldrich ${ }^{\circledR}$ (St. Louis, Missouri). 1,4,7,10-Tetraazacyclododecane-1,4,7-tris(t-butyl-acetate)-10(aminoethylacetamide) was purchased from Macrocyclics ${ }^{\circledR}$ Inc. (Plano, Texas). 1,2-dimyristoyl-snglycero-3-phosphocholine (DMPC) was purchased from Avanti® Polar Lipids, Inc. (Alabaster, Alabama).

\subsubsection{Polymer synthesis and characterization}

a) Polymer synthesis. SMA-EA copolymer was synthesized, purified, and characterized according to the procedure described in the literature ${ }^{74}$. SMA-EA-DOTA copolymer was synthesized, as described previously ${ }^{63}$. In short, $1 \mathrm{~g}$ of SMAnh, $0.435 \mathrm{~g}$ of 1,4,7,10-Tetraazacyclododecane1,4,7-tris(t-butyl-acetate)-10-(aminoethylacetamide) (amino-DOTA), and $1 \mathrm{~mL}$ of triethylamine were dissolved in $60 \mathrm{ml}$ of anhydrous NMP and heated to $70^{\circ} \mathrm{C}$ under continuous stirring (Step- 
1). The addition of an excess of ethanolamine in the presence of an extra $1 \mathrm{~mL}$ of triethylamine for 2 more hours at the same temperature in the same round bottom flask completed the nucleophilic ring-opening reaction (Step-2). The final product was precipitated using $1 \mathrm{M} \mathrm{HCl}$. To deprotect the chelator units, the polymer was dissolved in TFA and reacted for 2 hours at room temperature under gentle stirring (Step-3). Finally, the product was precipitated using diethyl ether and washed multiple times with deionized water prior to lyophilization. The 3-step reaction yielded about $600 \mathrm{mg}$. The reaction scheme is below in Figure 3.1.
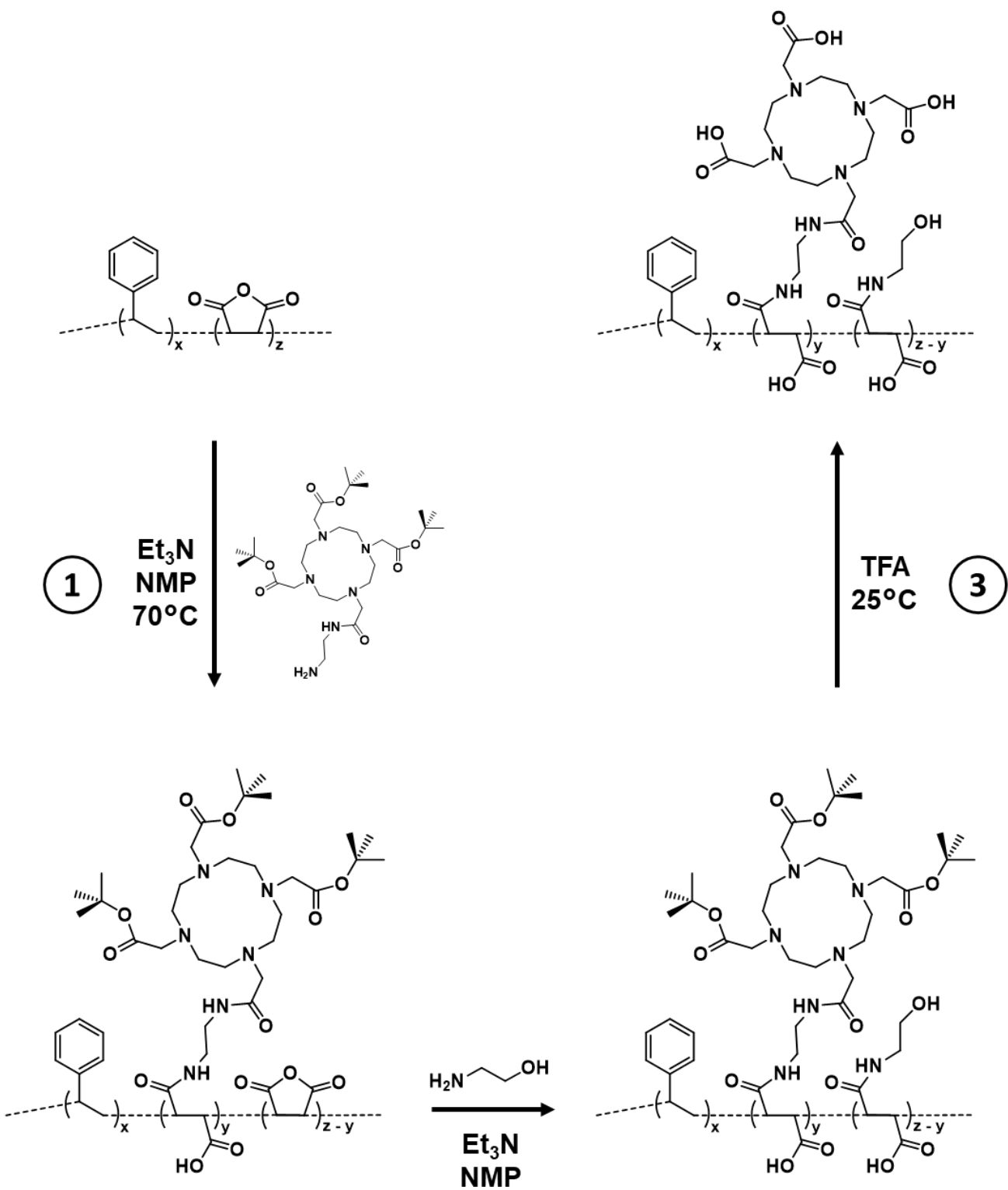

$70^{\circ} \mathrm{C}$

2

Figure 3.1. Reaction scheme for the synthesis of SMA-EA-DOTA: 1) The nucleophilic ring opening reaction using DOTA-t-butyl-acetate. 2) Nucleophilic ring opening reaction using ethanolamine. 3) TFA deprotection of DOTA. 
b) FT-IR spectroscopy. The newly synthesized polymer was characterized by FT-IR. Figure 3.2 shows (a) the full FT-IR spectra and (b) the $2000-1400 \mathrm{~cm}^{-1}$ expanded region reporting the $\mathrm{C}=\mathrm{O}$ stretching frequency of starting material, SMAnh (gray), and derived copolymers, SMA-EA (red) and SMA-EA-DOTA (blue). The shift of the carbonyl stretching frequency from $1770 \mathrm{~cm}^{-1}$ to $1702 \mathrm{~cm}^{-1}$ indicates the conversion of the anhydride to the amide, confirming the success of the reaction.
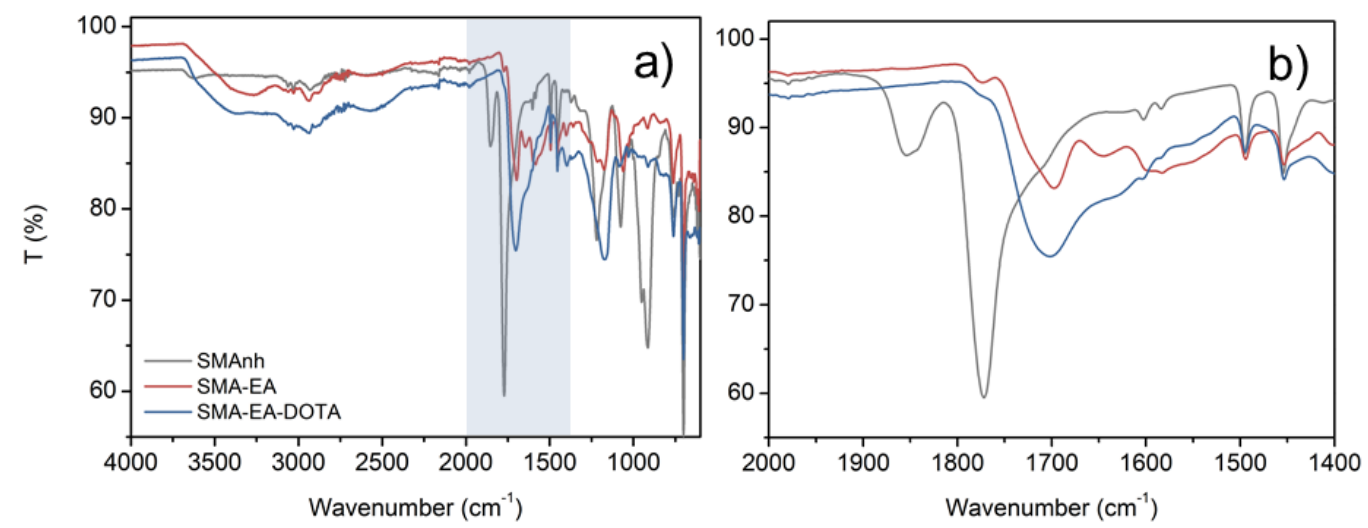

Figure 3.2. FT-IR spectra of copolymers. a) SMAnh (gray), SMA-EA (red), and SMA-EA-DOTA (blue); b) Expanded regions showing the $\mathrm{C}=\mathrm{O}$ stretching frequency $\left(2000-1400 \mathrm{~cm}^{-1}\right)$.

\subsubsection{Polymer nanodiscs preparation}

Stock solutions of each copolymer (SMA-EA and SMA-EA-DOTA) were obtained by dissolving the desired amount of powder in a $0.1 \mathrm{M} \mathrm{NaOH}$ solution. The $\mathrm{pH}$ was then adjusted to 7.4 using $1 \mathrm{M}$ $\mathrm{HCl}$. Polymer-based lipid nanodiscs were prepared by mixing the desired quantity of DMPC lipids and polymers in the ratio 1:1 by weight from $20 \mathrm{mg} / \mathrm{mL}$ stock solutions. Each sample was incubated overnight at room temperature prior to its use.

\subsubsection{Polymer nanodiscs characterization}

a) Size Exclusion Chromatography (SEC). Polymer-based phospholipid nanodiscs were purified by SEC using a self-packed Sephadex 200 16/600 column operated on a G.E. Healthcare ${ }^{\circledR}$ AKTA purifier. Samples were eluted at room temperature and at a flow rate of $1 \mathrm{~mL} / \mathrm{min}$. The elution was monitored using a UV-detector at $\lambda=254 \mathrm{~nm}$. Chromatograms are shown in Figure 3.5e.

b) Dynamic Light Scattering (DLS). All the DLS experiments were performed on a Wyatt Technology ${ }^{\circledR}$ DynaPro NanoStar ${ }^{\circledR}$ using a $1 \mu \mathrm{L}$ quartz MicroCuvette. The size distribution profiles for both DMPC: SMA-EA 1:1 w/w and DMPC: SMA-EA-DOTA 1:1 w/w polymer-lipid 
nanodiscs used in this study are reported in Figure 3.5f. Below, mass and intensity profiles are reported.

a)

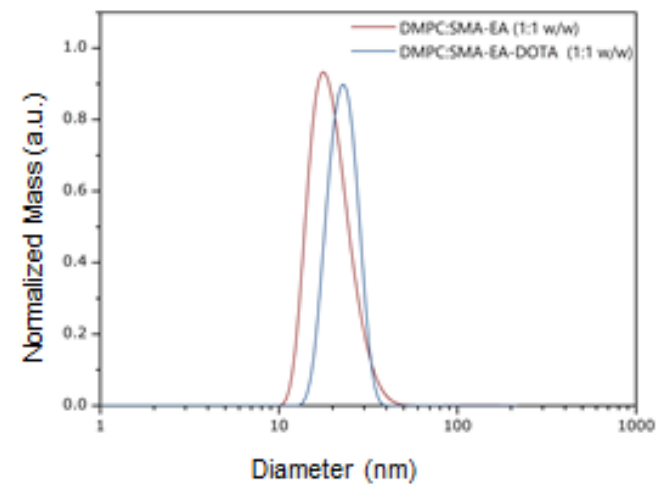

b)

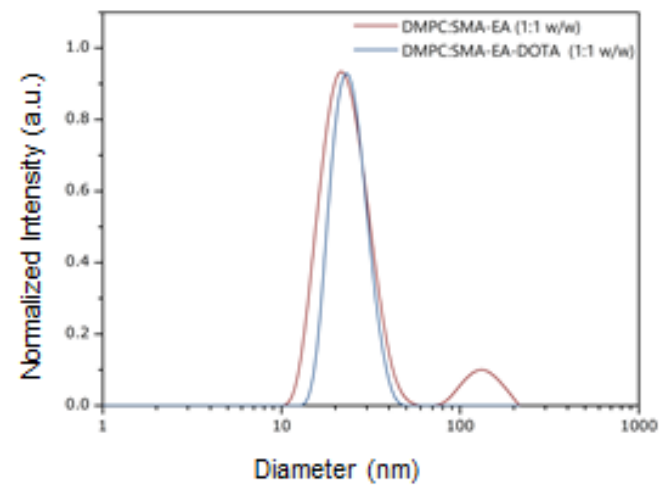

Figure 3.3. DLS of nanodiscs. DLS profiles of 1:1 w/w DMPC:SMA-EA (in red) and DMPC:SMA-EA-DOTA (in blue). a) The mass profiles, and b) intensity profiles. Both were obtained on samples passed through SEC.

c) Static Light Scattering (SLS). All the SLS experiments were performed using a $4 \mathrm{~mL}$ cuvette (1 $\mathrm{cm}$ optical path) under continuous stirring at $25^{\circ} \mathrm{C}$ on a FluoroMax-4® Spectrofluorometer from Horiba Scientific ${ }^{\circledR}$. The excitation wavelength was set at $400 \mathrm{~nm}$ while the emission wavelength was set at $404 \mathrm{~nm}$, and the slit was set to $2 \mathrm{~nm}$.

d) Solubilization Experiments. The time-dependent solubilization of the DMPC suspension in 10 mM HEPES buffer $50 \mathrm{mM} \mathrm{NaCl}$ was monitored by the intensity of scattered light at a $90^{\circ}$ angle. The solubilization power of two different polymers SMA-EA and SMA-EA-DOTA, was tested on a $1 \mathrm{mg} / \mathrm{mL}$ DMPC MLVs solution. The amount of polymer added was equivalent $(1: 1 \mathrm{w} / \mathrm{w}$ ratio) for all of them. Data are shown in Figure 3.5c.

e) Metal ion titrations. Polymer-based lipid nanodiscs stability was tested by titrating a $1 \mathrm{mg} / \mathrm{mL}$ solution of DMPC: copolymer 1:1 w/w nanodiscs in $10 \mathrm{mM}$ HEPES buffer at $\mathrm{pH}=7.40$ with a $4 \mathrm{M}$ solution of each metal. The detailed results are shown in the Figure 3.4 and summarized as a bar graph in Figure 3.5d. 

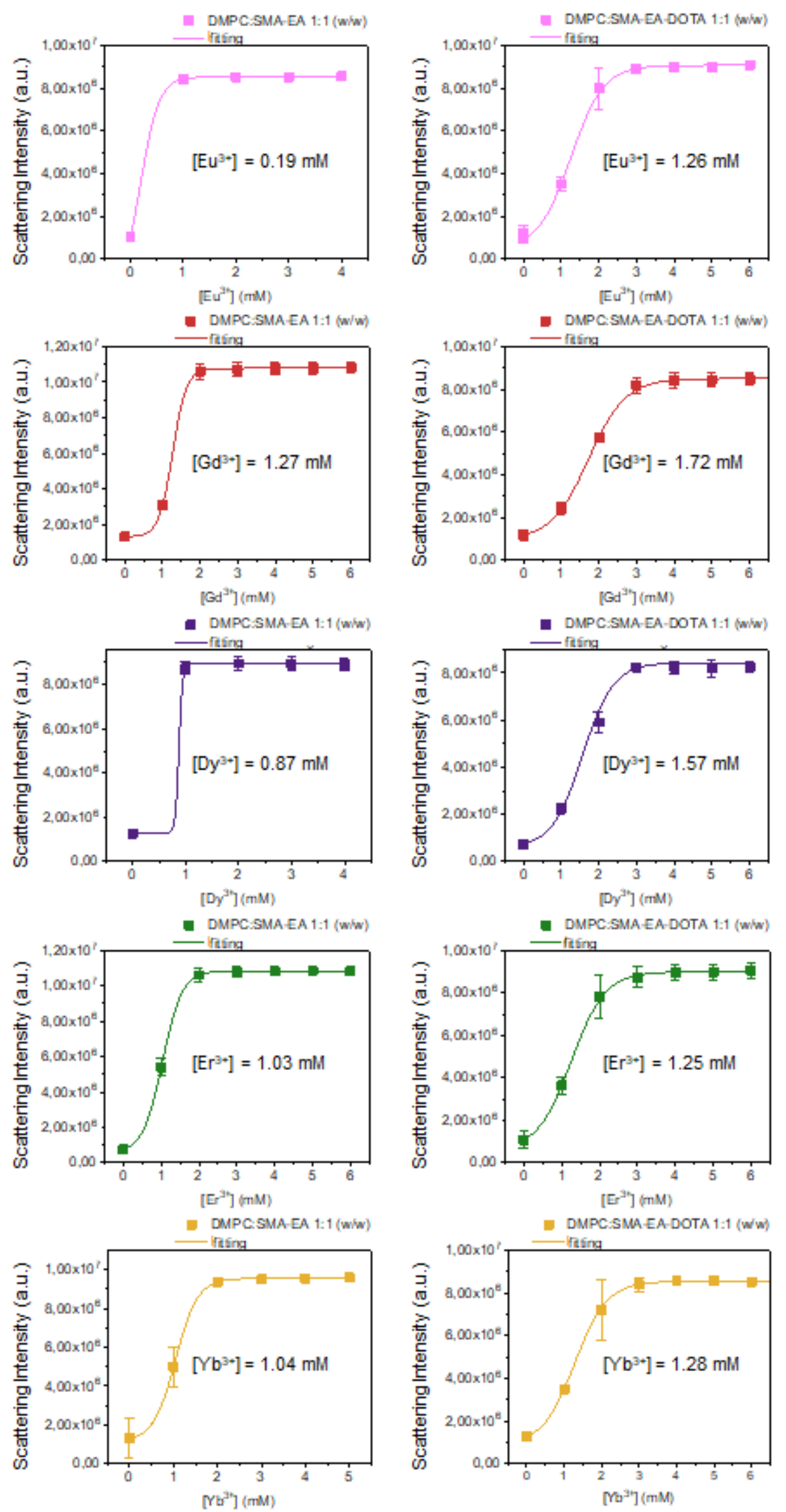

Figure 3.4. Static Light Scattering metal titrations. SLS traces obtained by titrating $1 \mathrm{mg}$ of SMA-EA nanodiscs (left column) and SMA-EA-DOTA nanodiscs (right column) with a solution of varying metal ion concentration. The concentration values were obtained by fitting the experimental points, obtained in triplicate. 
f) NMR Spectroscopy. Solution NMR experiments were performed at $11.75 \mathrm{~T}$ on a $500 \mathrm{MHz}$ Bruker Avance III HD spectrometer. NMR samples were prepared according to the procedure described in the "Nanodisc Formation" section and adding the desired concentration of metal ions $\left(0,5 \times 10^{-4}, 2.5 \times 10^{-2}, 5 \times 10^{-2}, 0.125,0.250,0.500,1.250\right.$, and $\left.2.500 \mathrm{mM}\right)$, then lyophilized for 24 hours prior to resuspension in $600 \mu \mathrm{L}$ of $\mathrm{D}_{2} \mathrm{O}$ and then transferred to $5 \mathrm{~mm}$ Norrell ${ }^{\circledR}$ Sample Vault Series ${ }^{\mathrm{TM}}$ glass tubes and placed in a commercial $5 \mathrm{~mm}$ quadruple resonance ${ }^{2} \mathrm{H} /{ }^{1} \mathrm{H} /{ }^{15} \mathrm{~N} /{ }^{13} \mathrm{C}$ Bruker round-coil TXITM $500 \mathrm{SB}$ probe. The experiments were performed in $\mathrm{D}_{2} \mathrm{O}$ at neutral $\mathrm{pH}$ at three different temperatures, 15,25 , and $35^{\circ} \mathrm{C}$. Each sample was made using $4 \mathrm{mg}$ of lipids, and an equal amount (by weight) of SMA-EA-DOTA polymer, titrated at $\mathrm{pH} 7.4$ to obtain a DMPC: SMA-EA-DOTA $(1: 1 \mathrm{w} / \mathrm{w})$ system. Each concentration of each paramagnetic metal investigated was prepared individually and tested on three sequential different experiments.

- $\quad{ }^{1} \mathrm{H}-\mathrm{NMR} .{ }^{1} \mathrm{H}$ spectra were recorded by collecting 32 scans with a spectral width of $25 \mathrm{ppm}$. The transmitter frequency offset was set at $4.7 \mathrm{ppm}$. Acquisition time and relaxation delay were respectively set at $0.8 \mathrm{~s}$ and $1.0 \mathrm{~s} .{ }^{1} \mathrm{H}-\mathrm{NMR}$ spectra for DMPC:SMA-EA 1:1 (w/w) and DMPC:SMA-EA-DOTA 1:1 (w/w) are shown in Figure 3.7c.

- Inversion recovery experiment. To measure $T_{1}$, an inversion recovery experiment was performed. Fifteen data points from $0.001 \mathrm{~s}$ to $10.0 \mathrm{~s}$ were collected by acquiring 8 scans with a spectral width of $10 \mathrm{ppm}$. The transmitter frequency offset was set at $4.7 \mathrm{ppm}$. Acquisition time and relaxation delay were respectively set at $0.001 \mathrm{~s}$ and $10 \mathrm{~s}$. For $\mathrm{Gd}^{3+}$-loaded DMPC:SMA-EA-DOTA $(1: 1 \mathrm{w} / \mathrm{w})$ nanodiscs experimental data are shown in Figures 3.7 and 8. $\mathrm{T}_{1}$ values are shown in Table 3.1 (Chapter 3). All the experimental data for $\mathrm{Gd}^{3+}$-loaded DMPC:SMA-EA (1:1 w/w) nanodiscs and for $\mathrm{Eu}^{3+}-/ \mathrm{Dy}^{3+}-/ \mathrm{Er}^{3+}-/ \mathrm{Yb}^{3+}$-loaded DMPC:SMAEA-DOTA (1:1 w/w) nanodiscs are shown in Figures S1-S5 and S7 (Appendix). $\mathrm{T}_{1}$ values are respectively shown in Tables S1-S5 (Appendix).

- Data Processing. Data have been processed using both Bruker Topspin ${ }^{\text {TM }} 3.2$ and Mestrelab Research S.L. MestReNova ${ }^{\mathrm{TM}}$ software was used to integrate the peaks of interest. 


\subsection{Results and Discussion}

Formation of SMA-EA-DOTA lipid-nanodiscs

SMA-EA-DOTA copolymer was synthesized according to the procedure, as briefly described above $^{63}$. Figure $3.5 \mathrm{a}$ shows the chemical structures of SMA-EA and SMA-EA-DOTA co-polymers. FT-IR characterization (Figure 3.5b) confirms the functionalization of SMA-EA and DOTA, as reported previously. ${ }^{63}$ The ability to solubilize 1,2-dimyristoyl-sn-glycero-3-phosphocholine (DMPC) lipid vesicles (or aggregates) was tested at room temperature $\left(\sim 25^{\circ} \mathrm{C}\right)$. For this study, about 20-25 nm size polymer-nanodiscs formed by the addition of a 1:1 lipids-to-polymer weight ratio (1:1 $\mathrm{w} / \mathrm{w}$ ) was used (Figure 3.5e and 3.5f). Data shown in Figure 3.5c confirms similar solubilization capabilities of SMA-EA and SMA-EA-DOTA copolymers. Since only a small number of DOTA units per polymer chain were introduced, both SMA-EA and SMA-EA-DOTA have comparable nanodiscs forming capabilities, as shown by the experimental results in Figure 3.5. Additionally, dynamic light scattering (DLS) profiles (Figure 3.5f) after size-exclusion chromatography (SEC) purification (Figure 3.5e) confirm the formation of nanodiscs and their isolation. These large-size nanodiscs (>20 nm) are also known as "macro-nanodiscs," which can be aligned in the presence of an external magnetic field as demonstrated in the previous studies and are useful for solid-state NMR studies. ${ }^{74}$

SMA-EA-DOTA nanodiscs are more stable than SMA-EA nanodiscs in the presence of metals

The stability of the polymer nanodiscs was examined using static light scattering (SLS) experiments as described above. The SLS experimental results shown in Figure 3.1d demonstrate the enhanced stability of DOTA containing polymer nanodiscs in the presence of metal ions. In addition to the above-mentioned FT-IR data (Figure 3.5b), the SLS results confirm the successful functionalization of SMA-EA with DOTA. Because of the presence of DOTA groups in SMA-EA-DOTA, the high binding affinity for various lanthanides renders a near-complete chelation and thus the high stability observed in the presence of various metal ions as shown in Figures 3.4 and $3.5 \mathrm{~d}^{75,76}$. 
a)

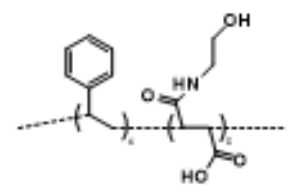

SMA-EA

c)

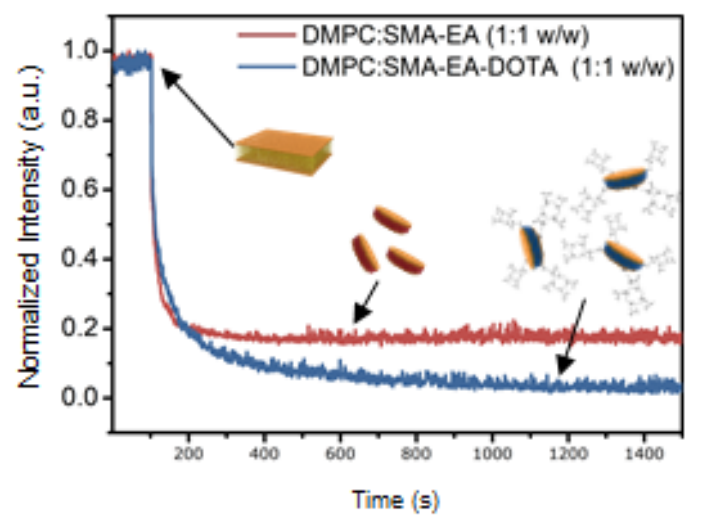

e)
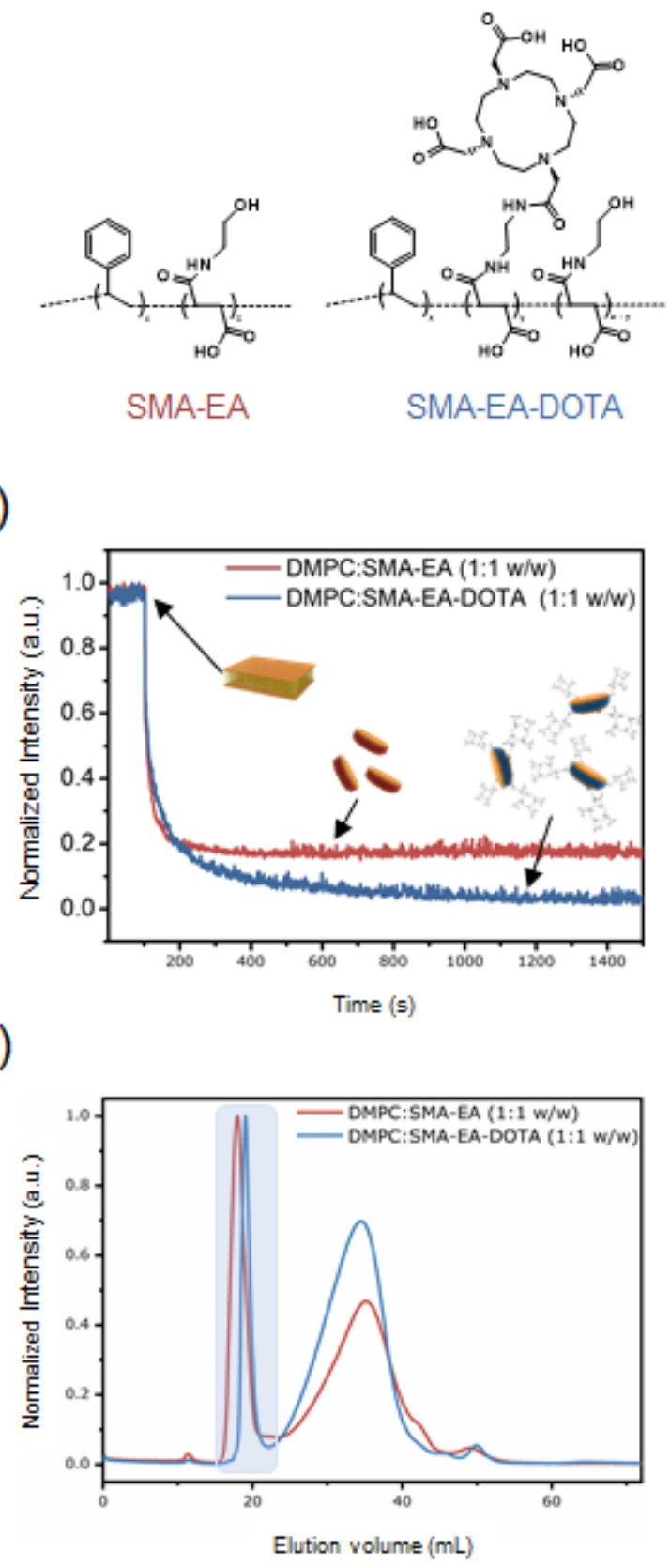

b)

d)
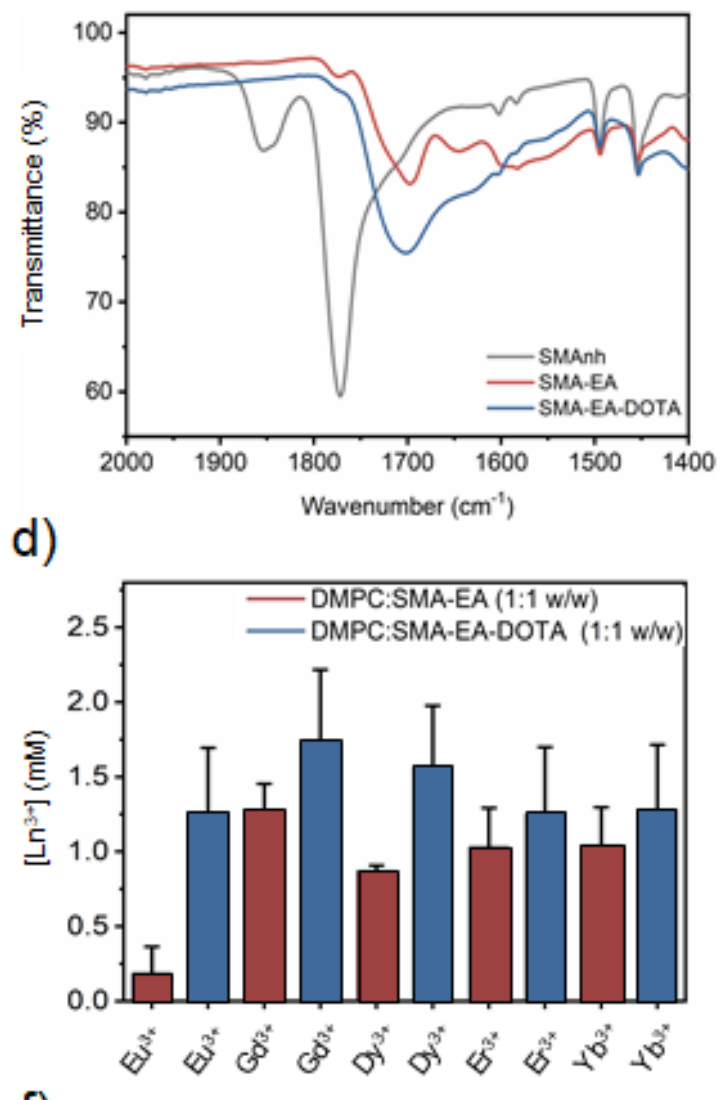

f)

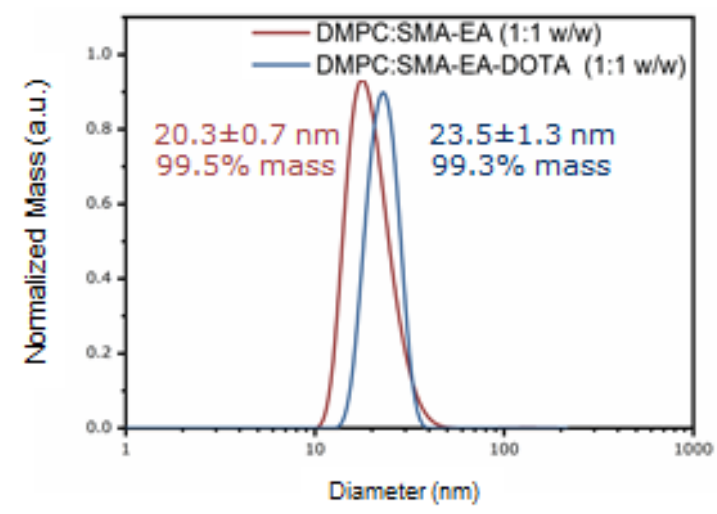

Figure 3.5. Characterization of polymers and polymer nanodiscs. a) Molecular structures of SMA-EA and SMA-EADOTA. b) FT-IR spectra of the starting material (SMAnh) and synthetic polymers. FT-IR results confirm the functionalization of the starting material (SMAnh in dark gray) and similarities among SMA-EA (in red) and SMA-EADOTA (in blue). Full spectra are included in Figure 3.2. c) Dissolution of multilamellar vesicles (MLVs) by SMA-EA (red) and by SMA-EA-DOTA (blue) for a 1:1 lipid:polymer weight ratio. d) Stability of DMPC:SMA-EA 1:1 w/w and DMPC: SMA-EA-DOTA 1:1 w/w macro-nanodiscs against different metal ions. Size exclusion chromatography (e) and DLS (f) profiles for 1:1 w/w ratio of DMPC:SMA-EA and DMPC:SMA-EA-DOTA samples. The DLS profiles were obtained after SEC purification for the nanodiscs fraction highlighted in (e). The intensity profile can be found in Figure 3.4 . 
Figure 3.6 shows a schematic representation of the chelation of paramagnetic cations to SMA-EADOTA copolymer nanodiscs and its use for rapid NMR data acquisition. A first successful application of $\mathrm{Cu}^{2+}$ based PRE-NMR with this polymer system was recently reported from our research group, which probed the interaction between polymer nanodiscs and G-quadruplex ${ }^{63}$. In this study, the use of paramagnetic properties of five additional trivalent cations from elements of the f-block of the periodic table $\left(\mathrm{Eu}^{3+}, \mathrm{Gd}^{3+}, \mathrm{Dy}^{3+}, \mathrm{Er}^{3+}, \mathrm{Yb}^{3+}\right)$ is investigated by measuring the spin-lattice $\left(\mathrm{T}_{1}\right)$ relaxation times of protons for various concentrations of the metal ions.

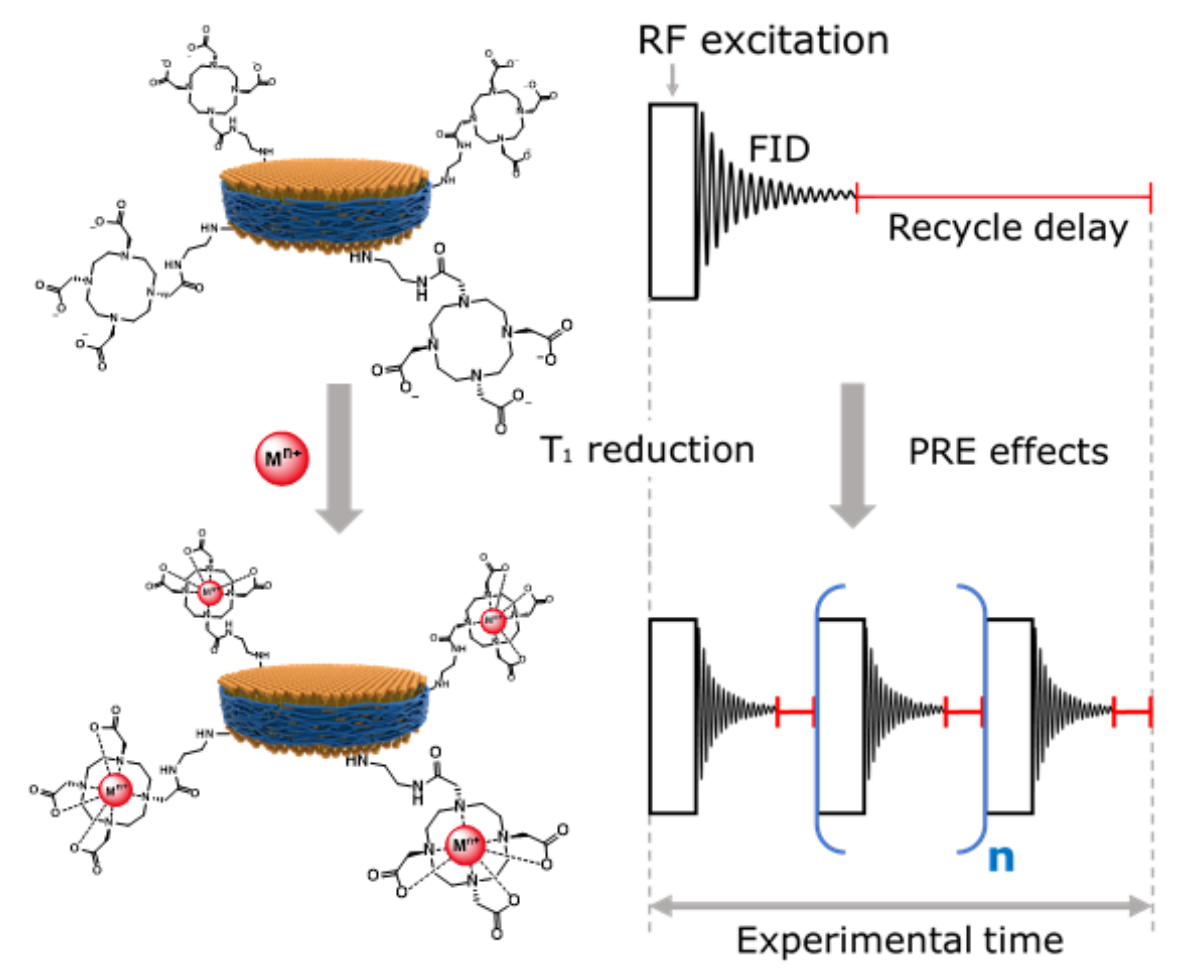

Figure 3.6. Use of paramagnetic metal-chelated polymers to speed-up NMR data acquisition. A schematic representation of how SMA-EA-DOTA copolymer-based nanodiscs chelated with paramagnetic metals can be used to reduce $T_{1}$ relaxation times and to shorten the recycle delay between the successive scans in NMR data acquisition.

Based on the assignment of NMR peaks as reported previously ${ }^{63,77}$, it is possible to assign the ${ }^{1} \mathrm{H}$ peaks observed from lipid and polymer components of nanodiscs: 1 ppm for protons from the lipid acyl $\left(-\mathrm{CH}_{3}\right), 1.4 \mathrm{ppm}$ for protons from the lipid acyl $\left(-\mathrm{CH}_{2}-\right), 3.3 \mathrm{ppm}$ for protons from the lipid head quaternary ammonium $\left(-\mathrm{CH}_{3}, \gamma\right)$, and $7.3 \mathrm{ppm}$ for protons from the aromatic styrene group of the polymer. The structure of DMPC and peak assignment of ${ }^{1} \mathrm{H}$ NMR spectra for both DMPC:SMA-EA and DMPC:SMA-EA-DOTA polymer-based nanodiscs are shown in Figure 3.7c. The various paramagnetic metals used in the present study $\left(\mathrm{Eu}^{3+}, \mathrm{Gd}^{3+}, \mathrm{Dy}^{3+}, \mathrm{Er}^{3+}, \mathrm{Yb}^{3+}\right)$ were chosen among the 14 f-block elements to be representative of the magnetic differences reported in the literature ${ }^{52,70-}$ $72,77-80$. 
a)

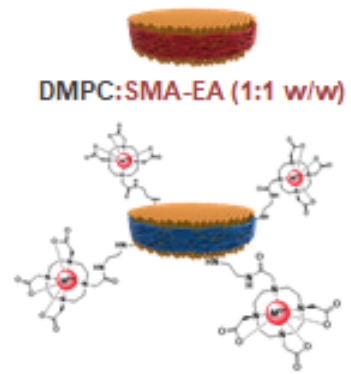

DMPC:SMA-EA-DOTA $(1: 1$ w/w)

b)

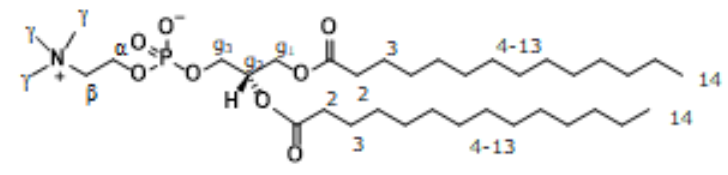

Dimiristoyl-sn-glycero-phosphocholine (DMPC)

c)

DMPC:SMA-EA (1:1 w/w)
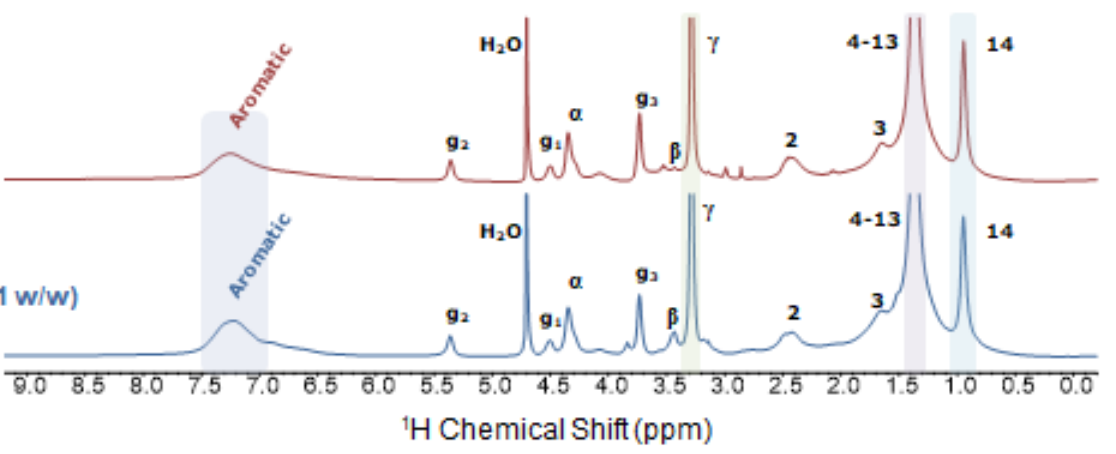

d)

DMPC:SMA-EA-DOTA (1:1 w/w)
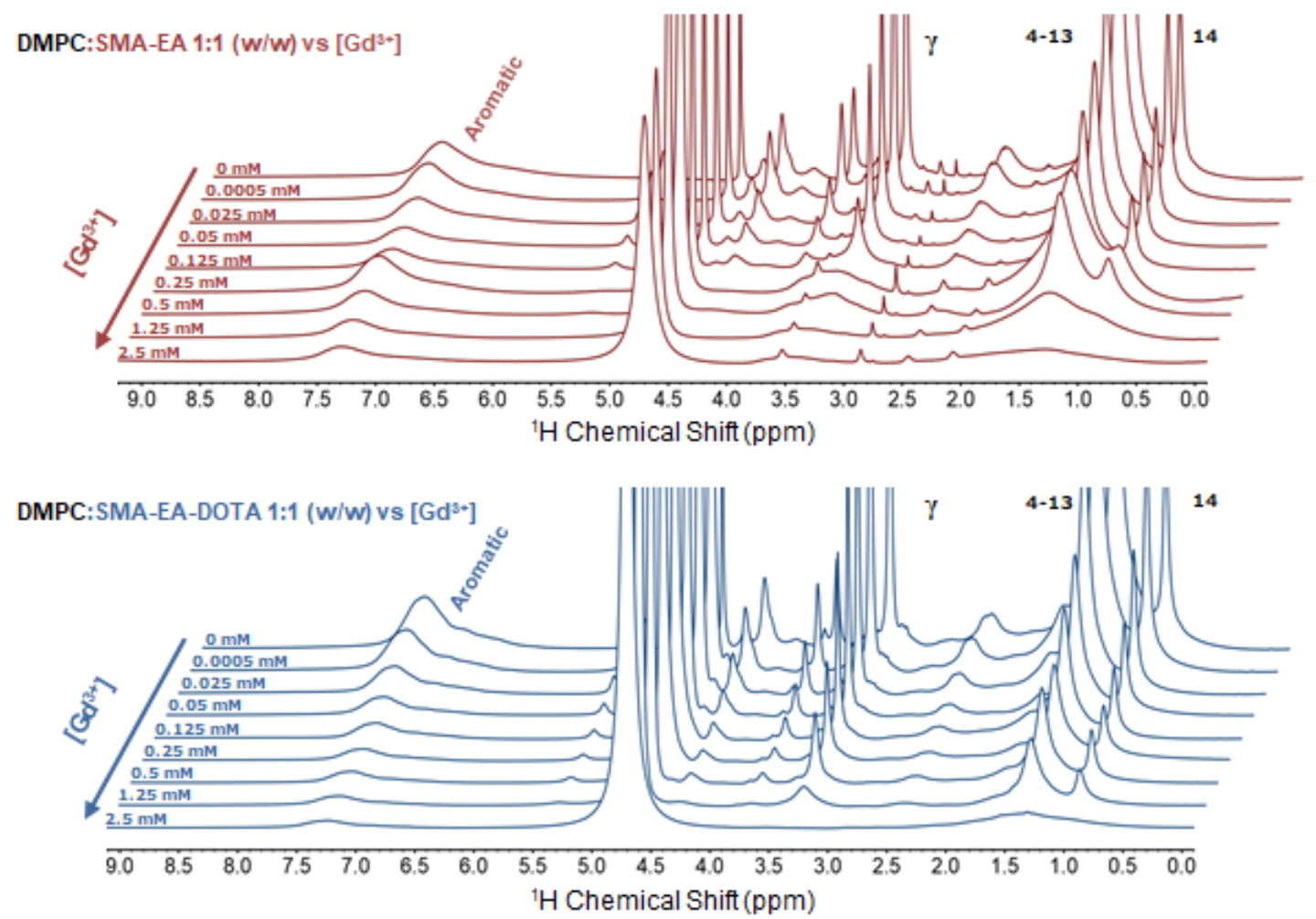

Figure 3.7. NMR spectra of nanodises in the presence of $\mathbf{G d}^{3+}$ ions. a) Schematic representation of a macro-nanodisc.

b) Molecular structure of 1,2-dimyristoyl-sn-glycero-3-phosphocholine (DMPC). c) ${ }^{1} \mathrm{H}$ NMR spectra of 1:1 w/w DMPC:SMA-EA (red) and DMPC:SMA-EA-DOTA (blue) macro-nanodiscs. d) ${ }^{1} \mathrm{H}$ NMR spectra of 1:1 w/w DMPC: SMA-EA (red) and SMA-EA-DOTA (blue) nanodiscs titrated with the indicated amount of $\mathrm{Gd}^{3+}$ ions. All NMR were obtained using $4 \mathrm{mg}$ of lipids in $10 \mathrm{mM}$ phosphate buffer $(\mathrm{pH}=7.4)$ in $100 \% \mathrm{D}_{2} \mathrm{O}$ at $35^{\circ} \mathrm{C}$. 
Figure 3.7d shows ${ }^{1} \mathrm{H}$ NMR spectra of SMA-EA and SMA-EA-DOTA nanodiscs containing DMPC lipids and the indicated concentration of $\mathrm{Gd}^{3+}$ ions. Here, using two different types of nanodisc systems (with and without the DOTA) of comparable sizes (Figures 3.5e and 3.5f), one noticeable difference we observed was that significant line-broadening occurred at a much lower $\mathrm{Gd}^{3+}$ ions concentration $(0.25 \mathrm{mM})$ for the SMA-EA polymer nanodiscs as compared to that observed for SMAEA-DOTA. The severe line-broadening observed for the lipid headgroup's methyl protons of SMAEA polymer nanodiscs is most likely due to the presence of a higher population of free $\mathrm{Gd}^{3+}$ ions that directly bind to the zwitterionic lipid headgroup. On the other hand, the SMA-EA-DOTA polymer containing nanodiscs attracts the $\mathrm{Gd}^{3+}$ ions from the sample to be chelated to the DOTA unit on the polymer belt of the nanodisc and avoid the line-broadening effects. The inversion-recovery NMR experiments were performed to measure the $T_{1}$ values of protons in order to examine the effect of the added paramagnetic metal ions in the sample (see Materials and Methods). The $\mathrm{T}_{1}$ values were measured by integrating the observed ${ }^{1} \mathrm{H}$ NMR peaks of interest and then fitting the experimentally measured data to the following equation:

$$
M_{z}(\tau)=M_{z, \text { eq }}\left(1-2 e^{-\tau / T_{1}}\right)
$$

Figure 3.8 shows the fitting curves obtained for the 1:1 w/w DMPC:SMA-EA-DOTA nanodiscs sample for various concentrations of $\mathrm{Gd}^{3+}$ ions.
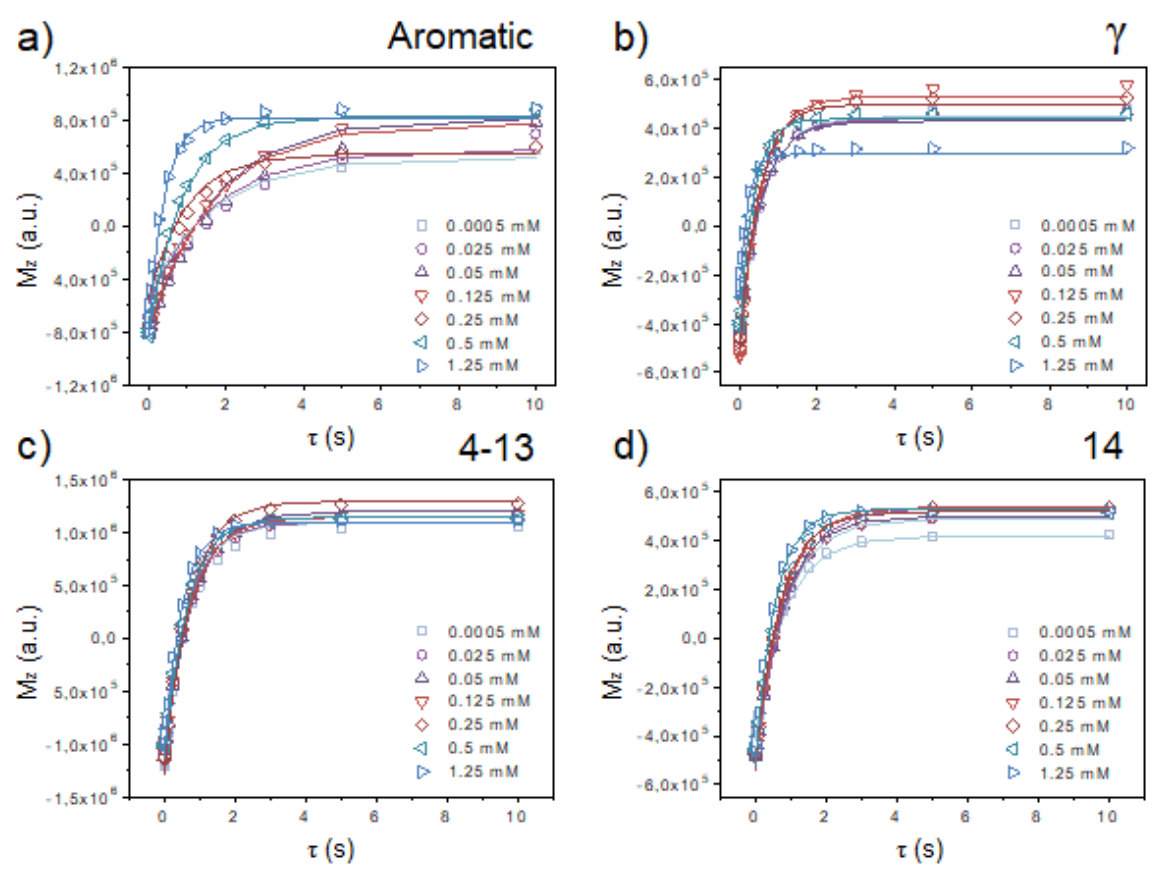

Figure 3.8. Measurement of $\mathbf{T}_{\mathbf{1}}$ for protons. Spin-inversion NMR experimental data obtained from 1:1 w/w DMPC:SMA-EA-DOTA nanodiscs to determine $\mathrm{T}_{1}$ values of protons for varying concentrations of $\mathrm{Gd}^{3+}$ as indicated. Equation 1 was used to obtain the best-fitting values given in Table 1 . 


\begin{tabular}{|c|cccc|}
\cline { 2 - 5 } \multicolumn{1}{c|}{} & \multicolumn{4}{c|}{${ }^{1}$ H Peaks } \\
\hline$\left.\left[\mathbf{G d}^{3+}\right] \mathbf{( m M}\right)$ & Aromatic (s) & $\boldsymbol{\gamma}(\mathbf{s})$ & $\mathbf{4 - 1 3}(\mathbf{s})$ & $\mathbf{1 4}(\mathbf{s})$ \\
\hline 0 & 1.65 & 0.59 & 0.70 & 0.80 \\
0.0005 & 1.70 & 0.60 & 0.70 & 0.85 \\
0.025 & 1.70 & 0.58 & 0.67 & 0.83 \\
0.05 & 1.70 & 0.56 & 0.77 & 0.87 \\
0.125 & 1.70 & 0.53 & 0.72 & 0.79 \\
0.25 & 0.97 & 0.49 & 0.70 & 0.76 \\
0.5 & 0.89 & 0.35 & 0.65 & 0.70 \\
1.25 & 0.40 & 0.20 & 0.50 & 0.55 \\
2.5 & N/D & N/D & N/D & N/D \\
\hline
\end{tabular}

Table 3.1. $T_{1}$ values of DMPC: SMA-EA-DOTA 1:1 $(\mathrm{w} / \mathrm{w})$ macro-nanodises for varying $\left[\mathbf{G d}^{3+}\right]$. Values marked as N/D are data not determined due to significant line-broadening because of the presence of paramagnetic ions.

As a control experiment, the resulting $\mathrm{T}_{1}$ of the four-aforementioned peaks (aromatic, $\gamma$, methylene, and methyl groups) is plotted against $\left[\mathrm{Gd}^{3+}\right]$ for DMPC:SMA-EA $(1: 1 \mathrm{w} / \mathrm{w})$ polymer-nanodiscs samples in Figure S1 (Appendix). These experiments were also carried out for the other paramagnetic metal ions, and the results are included as supporting information in the Appendix (Figures S2-S5). Figure 3.9 shows the $\mathrm{T}_{1}$ values for the four ${ }^{1} \mathrm{H}$ peaks of interest obtained for the different metal ions. At $\left[\mathrm{Ln}^{3+}\right]=0.5 \mathrm{mM}, \mathrm{Gd}^{3+}$, followed by $\mathrm{Dy}^{3+}$, showed the largest $\mathrm{T}_{1}$-reduction for all of the peaks as compared to a diamagnetic reference system made up of DMPC:SMA-EA-DOTA 1:1 (w/w). The aromatic peak from the styrene fraction of the polymer is the most affected by the paramagnetic ions due to the proximity of the styrene group to the DOTA unit's chelated metals on the nanodiscs belt. $\mathrm{Gd}^{3+}$ and $\mathrm{Dy}^{3+}$ ions show $\mathrm{T}_{1}$ times reduced respectively by 1.9- and 1.3-times. Even though $\mathrm{Gd}^{3+}$ ions provided the greatest $T_{1}$ relaxation effect at the lowest concentration among all the investigated metals, other metal ions, like $\mathrm{Dy}^{3+}$, had little to no significant line-broadening effect up to $1.25 \mathrm{mM}$ (Figure S2 and Table S2). Metal ions such as $\mathrm{Er}^{3+}, \mathrm{Eu}^{3+}$, and $\mathrm{Yb}^{3+}$ showed significant line-broadening at the highest concentration investigated $(2.5 \mathrm{mM})$, but no effects on $\mathrm{T}_{1}$-reduction were observed (Figures S3-S5 and Tables S3-S5). Data for $\left[\mathrm{Er}^{3+}\right]=2.5 \mathrm{mM}$ are not shown because the sample showed instability.

NMR peaks from the DMPC lipid are also $\mathrm{T}_{1}$ enhanced by the presence of the paramagnetic tags on the belt but to a lesser extent, especially for $\mathrm{Eu}^{3+}, \mathrm{Er}^{3+}$, and $\mathrm{Yb}^{3+}$ ions (Figure 3.9). Particularly $\mathrm{Gd}^{3+}$ ions shorten $\mathrm{T}_{1}$ values of protons from $\gamma$, methylene (C4-C13), and methyl-groups (C14) by respectively $\sim 48 \%, \sim 40 \%, \sim 10 \%$, and $\sim 12 \%$, respectively. While line-broadening was observed for $\mathrm{Gd}^{3+}$ ions, the effects observed for other metals at $0.5 \mathrm{mM}$ are on the following order: $\mathrm{Eu}^{3+}<\mathrm{Yb}^{3+}<$ $\mathrm{Er}^{3+}<\mathrm{Dy}^{3+} \cdot \mathrm{Eu}^{3+}$ and $\mathrm{Yb}^{3+}$ do not show any $\mathrm{T}_{1}$-reduction. $\mathrm{Er}^{3+}$ ions showed an important paramagnetic relaxation enhancement on the aromatic peak from styrene groups $\left(\mathrm{T}_{1}\right.$-reduction of 
$\sim 35 \%$ at a $\left[\mathrm{Er}^{3+}\right]=0.5 \mathrm{mM}$ ) but weak-to-negligible effects for $\gamma$, methylene (4-13), and methyl (14) peaks. The fact that DMPC's acyl chains are less affected by the paramagnetic tags may be attributed to the large size of the nanodisc investigated) $\sim 20 \mathrm{~nm}$. We expect the PRE effect to be higher for very large macro-nanodiscs under high viscous conditions as they do not tumble fast, and therefore the unaveraged ${ }^{1} \mathrm{H}-{ }^{1} \mathrm{H}$ dipolar couplings should aid the PRE effect from the metals present on the belt. Such results have been observed for large bicelles that align magnetically ${ }^{81}$. Overall, the effect of paramagnetic metals on shortening $\mathrm{T}_{1}$ can be ranked as $\mathrm{Gd}^{3+}>\mathrm{Dy}^{3+}>\mathrm{Er}^{3+}>\mathrm{Eu}^{3+}>\mathrm{Yb}^{3+}$. Figures S2S5 show the stacking of $1 \mathrm{D}{ }^{1} \mathrm{H}$ NMR spectra and the $\mathrm{T}_{1}$ fitting curves. Tables $\mathrm{S} 2-\mathrm{S} 5$ report the experimentally measured $\mathrm{T}_{1}$ values.
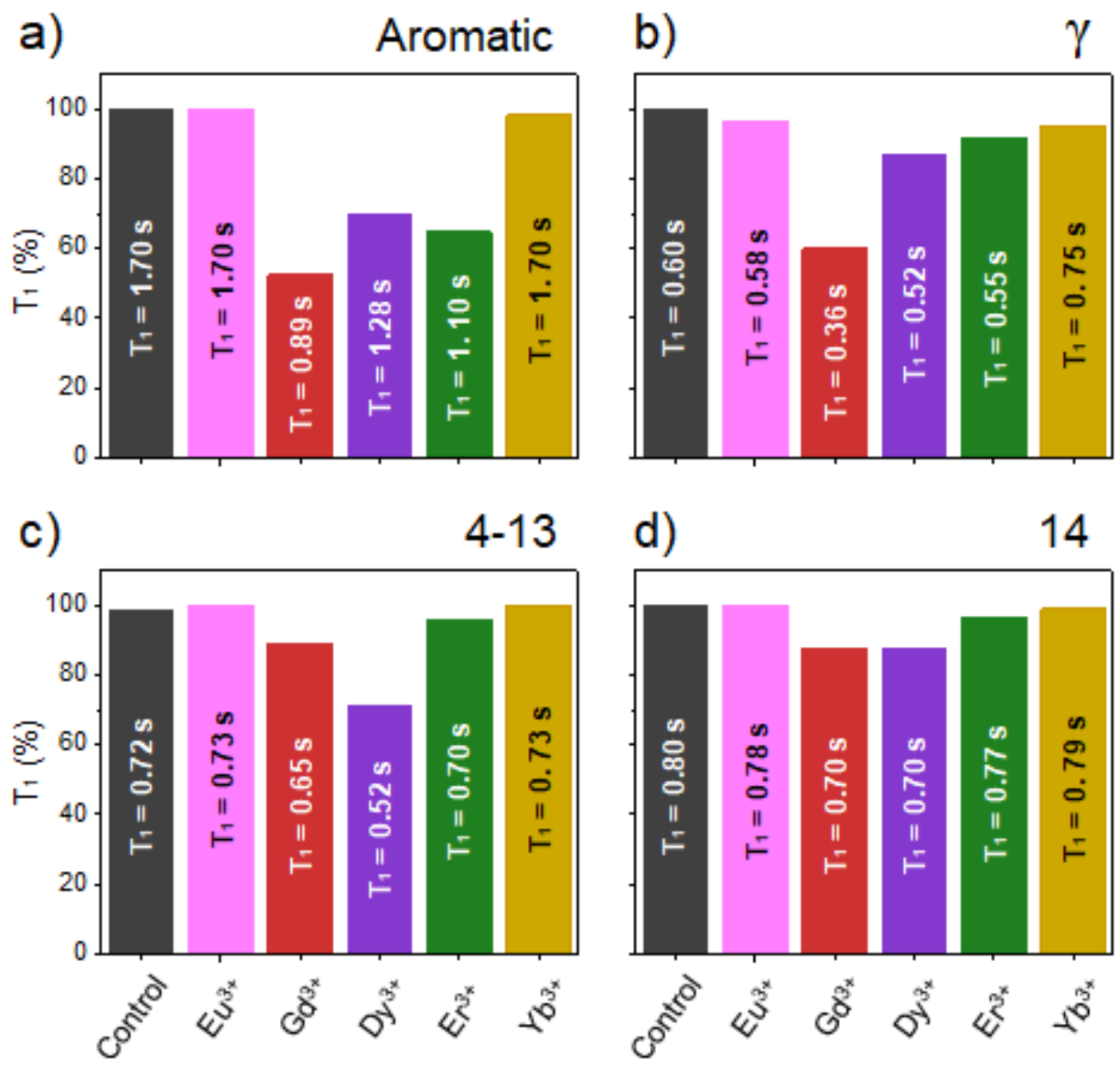

Figure 3.9. Efficiency of paramagnetic metal ions in shortening $T_{1}$. $T_{1}$ comparison among the different investigated metal ions at $\left[\mathrm{Ln}^{3+}\right]=0.5 \mathrm{mM}$. The black bar represents the data obtained from a control sample, 1:1 (w/w) DMPC: SMAEA-DOTA without paramagnetic metal ions. Data shown for ${ }^{1} \mathrm{H}$ peaks of a) styrene/aromatic group, b) $\gamma$ methyl groups in the quaternary ammonium of DMPC, c) the methylene groups from the acyl chains (C4-C13) of DMPC, and d) the terminal acyl-chain methyl groups (C14) of DMPC. Each of the NMR samples used in these measurements consisted of $4 \mathrm{mg}$ of DMPC in $10 \mathrm{mM}$ Phosphate buffer $\mathrm{pH}=7.4$ in $\mathrm{D}_{2} \mathrm{O}$. 
Because of the large size of the nanodiscs, within the spectral resolution, the observed proton NMR spectra do not show any shift in the observed resonance frequency when the nanodiscs are loaded with any of the lanthanides even for those that are considered as "shifting-agents" such as $\mathrm{Eu}^{3+}, \mathrm{Dy}^{3+}$, $\mathrm{Er}^{3+54,82}$. Spectra and full titrations with the fitting of the experimental data are included in the Materials and Methods section, Figure 3.4.

\subsection{Conclusions}

SMA-based nanodiscs are a great innovation in biochemistry and biophysics and are widely used as a membrane-mimicking system to investigate membrane proteins through several techniques. NMR spectroscopy, as a valuable non-disruptive technique, offers structural and dynamic information. Unfortunately, limitations such as its intrinsically low sensitivity result in long acquisition times to enhance the signal-to-noise ratio of NMR spectra. PRE can be used to reduce the acquisition times effectively but involves the introduction of external probes in the system and may cause undesired line-broadening in the spectra when compared to the diamagnetic counterpart. To overcome these limitations, we have demonstrated the efficiency of SMA-EA-DOTA copolymer in $\mathrm{T}_{1}$-reduction. This modification of SMA-EA copolymer allows the use of PRE effects in nanodiscs samples. Particularly, the introduction of chelating units that strongly bind paramagnetic metals on the outer belt of the nanodiscs avoids the addition of paramagnetic dopants directly in buffer solutions, in the membrane protein of interest, and in the bilayer components. SMA-EA-DOTA-nanodiscs represent a much less invasive approach toward the preservation of the integrity of the sample, offering PRE effects in a native-like environment.

As demonstrated in this study, this approach can be used to speed up NMR data acquisition (up to $\sim 50 \%$ ) with minimum-to-no alteration of the spectral quality due to spin-spin relaxation enhancement. A comparison of the effects of different paramagnetic metals shows that $\mathrm{Gd}^{3+}$ and $\mathrm{Dy}^{3+}$ can be successfully used to shorten $T_{1}$, and so, the recycle delay of NMR experiments. We believe that these results can broaden the applications of polymer-nanodiscs in the investigation of membrane proteins in a native-like environment, using both solution and solid-state NMR spectroscopy. Additionally, paramagnetically-labeled nanodiscs can be used for both dynamic nuclear polarization (DNP) NMR and electron paramagnetic resonance (EPR) studies. Both techniques require the presence of paramagnetic tags and could benefit from this improved version of polymer-nanodiscs. 


\section{Acknowledgments}

This research was supported by the National Institute of Health (NIH, GM084018 to A.R.). We thank

Dr. Kocman for the help with NMR experiments and Dr. Ravula for synthesizing and characterizing the SMA-EA copolymer used in this study. 


\section{References}

(1) Denisov, I. G.; Sligar, S. G. Nanodiscs in Membrane Biochemistry and Biophysics. Chemical Reviews. 2017, 4669-4713.

(2) Rawson, S.; Davies, S.; Lippiat, J. D.; Muench, S. P. The Changing Landscape of Membrane Protein Structural Biology through Developments in Electron Microscopy. Mol. Membr. Biol. 2016, 33 (1-2), 12-22.

(3) Dürr, U. H. N.; Gildenberg, M.; Ramamoorthy, A. The Magic of Bicelles Lights up Membrane Protein Structure. Chem. Rev. 2012, 112 (11), 6054-6074.

(4) Bayburt, T. H.; Grinkova, Y. V; Sligar, S. G. Self-Assembly of Discoidal Phospholipid Bilayer Nanoparticles with Membrane Scaffold Proteins. Nano Lett. 2002, 2 (8), 853-856.

(5) Knowles, T. J.; Finka, R.; Smith, C.; Lin, Y. P.; Dafforn, T.; Overduin, M. Membrane Proteins Solubilized Intact in Lipid Containing Nanoparticles Bounded by Styrene Maleic Acid Copolymer. J. Am. Chem. Soc. 2009, 131 (22), 7484-7485.

(6) Gulati, S.; Jamshad, M.; Knowles, T. J.; Morrison, K. A.; Downing, R.; Cant, N.; Collins, R.; Koenderink, J. B.; Ford, R. C.; Overduin, M.; et al. Detergent-Free Purification of ABC (ATPBinding-Cassette) Transporters. Biochem. J 2014, 461, 269-278.

(7) Swainsbury, D. J. K.; Scheidelaar, S.; Foster, N.; van Grondelle, R.; Killian, J. A.; Jones, M. R. The Effectiveness of Styrene-Maleic Acid (SMA) Copolymers for Solubilisation of Integral Membrane Proteins from SMA-Accessible and SMA-Resistant Membranes. Biochim. Biophys. Acta - Biomembr. 2017, 1859 (10), 2133-2143.

(8) Swainsbury, D. J. K.; Proctor, M. S.; Hitchcock, A.; Cartron, M. L.; Qian, P.; Martin, E. C.; Jackson, P. J.; Madsen, J.; Armes, S. P.; Hunter, C. N. Probing the Local Lipid Environment of the Rhodobacter Sphaeroides Cytochrome Bc 1 and Synechocystis Sp. PCC 6803 Cytochrome b 6 f Complexes with Styrene Maleic Acid. Biochim. Biophys. Acta - Bioenerg. 
2018, 1859 (3), 215-225.

(9) Dörr, J. M.; Koorengevel, M. C.; Schäfer, M.; Prokofyev, A. V; Scheidelaar, S.; van der Cruijsen, E. A. W.; Dafforn, T. R.; Baldus, M.; Killian, J. A. Detergent-Free Isolation, Characterization, and Functional Reconstitution of a Tetrameric $\mathrm{K}^{+}$Channel: The Power of Native Nanodiscs. Proc. Natl. Acad. Sci. U. S. A. 2014, 111 (52), 18607-18612.

(10) Dörr, J. M.; Scheidelaar, S.; Koorengevel, M. C.; Dominguez, J. J.; Schäfer, M.; van Walree, C. A.; Killian, J. A. The Styrene-Maleic Acid Copolymer: A Versatile Tool in Membrane Research. Eur. Biophys. J. 2016, 45 (1), 3-21.

(11) Bayburt, T. H.; Carlson, J. W.; Sligar, S. G. Reconstitution and Imaging of a Membrane Protein in a Nanometer-Size Phospholipid Bilayer. J. Struct. Biol. 1998, 123 (1), 37-44.

(12) Denisov, I. G.; Sligar, S. G. Nanodiscs for Structural and Functional Studies of Membrane Proteins. Nat. Struct. Mol. Biol. 2016, 23 (6), 481-486.

(13) Hagn, F.; Nasr, M. L.; Wagner, G. Assembly of Phospholipid Nanodiscs of Controlled Size for Structural Studies of Membrane Proteins by NMR. Nat. Publ. Gr. 2017, 13.

(14) Toraya, S.; Nishimura, K.; Naito, A. Dynamic Structure of Vesicle-Bound Melittin in a Variety of Lipid Chain Lengths by Solid-State NMR. Biophys. J. 2004, 87 (5), 3323-3335.

(15) Stroud, Z.; Hall, S. C. L.; Dafforn, T. R. Purification of Membrane Proteins Free from Conventional Detergents: SMA, New Polymers, New Opportunities and New Insights. Methods 2018, 147, 106-117.

(16) Dominguez Pardo, J. J.; Dörr, J. M.; Iyer, A.; Cox, R. C.; Scheidelaar, S.; Koorengevel, M. C.; Subramaniam, V.; Killian, J. A. Solubilization of Lipids and Lipid Phases by the StyreneMaleic Acid Copolymer. Eur. Biophys. J. 2017, 46 (1), 91-101.

(17) Oluwole, A. O.; Danielczak, B.; Meister, A.; Babalola, J. O.; Vargas, C.; Keller, S. Solubilization of Membrane Proteins into Functional Lipid-Bilayer Nanodiscs Using a Diisobutylene/Maleic Acid Copolymer. Angew. Chemie - Int. Ed. 2017, 56 (7), 1919-1924.

(18) Danielczak, B.; Meister, A.; Keller, S. Influence of $\mathrm{Mg}^{2+}$ and $\mathrm{Ca}^{2+}$ on Nanodisc Formation by Diisobutylene/Maleic Acid (DIBMA) Copolymer. Chem. Phys. Lipids 2019, 221, 30-38.

(19) Lee, S. C.; Knowles, T. J.; Postis, V. L. G.; Jamshad, M.; Parslow, R. A.; Lin, Y. P.; Goldman, A.; Sridhar, P.; Overduin, M.; Muench, S. P.; et al. A Method for Detergent-Free Isolation of Membrane Proteins in Their Local Lipid Environment. Nat. Protoc. 2016, 11 (7), 1149-1162. 
(20) Rajesh, S.; Knowles, T.; Overduin, M. Production of Membrane Proteins without Cells or Detergents. N. Biotechnol. 2011, 28 (3), 250-254.

(21) Overduin, M.; Klumperman, B. Advancing Membrane Biology with Poly(Styrene-Co-Maleic Acid)-Based Native Nanodiscs. Eur. Polym. J. 2019, 110 (June 2018), 63-68.

(22) Yasuhara, K.; Arakida, J.; Ravula, T.; Ramadugu, S. K.; Sahoo, B.; Kikuchi, J. I.; Ramamoorthy, A. Spontaneous Lipid Nanodisc Fomation by Amphiphilic Polymethacrylate Copolymers. J. Am. Chem. Soc. 2017, 139 (51), 18657-18663.

(23) Hardin, N. Z.; Ravula, T.; Mauro, G. Di; Ramamoorthy, A. Hydrophobic Functionalization of Polyacrylic Acid as a Versatile Platform for the Development of Polymer Lipid Nanodisks. Small 2019, 15 (9), 1-5.

(24) Barnaba, C.; Sahoo, B. R.; Ravula, T.; Medina-Meza, I. G.; Im, S.-C.; Anantharamaiah, G. M.; Waskell, L.; Ramamoorthy, A. Cytochrome-P450-Induced Ordering of Microsomal Membranes Modulates Affinity for Drugs. Angew. Chemie 2018, 130 (13), 3449-3453.

(25) Ravula, T.; Hardin, N. Z.; Ramadugu, S. K.; Ramamoorthy, A. P.H. Tunable and Divalent Metal Ion Tolerant Polymer Lipid Nanodiscs. Langmuir 2017, 33 (40), 10655-10662.

(26) Ravula, T.; Hardin, N. Z.; Bai, J.; Im, S. C.; Waskell, L.; Ramamoorthy, A. Effect of Polymer Charge on Functional Reconstitution of Membrane Proteins in Polymer Nanodiscs. Chem. Commun. 2018, 54 (69), 9615-9618.

(27) Lindhoud, S.; Carvalho, V.; Pronk, J. W.; Aubin-Tam, M. E. SMA-SH: Modified StyreneMaleic Acid Copolymer for Functionalization of Lipid Nanodiscs. Biomacromolecules 2016, 17 (4), 1516-1522.

(28) Fujiwara, T.; Ramamoorthy, A. How Far Can the Sensitivity of NMR Be Increased? Annu. Reports NMR Spectrosc. 2006, 58, 155-175.

(29) Verardi, R.; Traaseth, N. J.; Masterson, L. R.; Vostrikov, V. V; Veglia, G. Isotope Labeling for Solution and Solid-State NMR Spectroscopy of Membrane Proteins. In Isotope labeling in Biomolecular NMR; Atreya, H. S., Ed.; Springer Netherlands: Dordrecht, 2012; pp 35-62.

(30) Tugarinov, V.; Kanelis, V.; Kay, L. E. Isotope Labeling Strategies for the Study of HighMolecular-Weight Proteins by Solution NMR Spectroscopy. Nat. Protoc. 2006, 1 (2), 749754.

(31) Lacabanne, D.; Meier, B. H.; Böckmann, A. Selective Labeling and Unlabeling Strategies in 
Protein Solid-State NMR Spectroscopy. J. Biomol. NMR 2018, 71 (3), 141-150.

(32) Opella, S. J.; Marassi, F. M. Structure Determination of Membrane Proteins by NMR Spectroscopy. Chem. Rev. 2004, 104, 3587-3606.

(33) Lee, J. H.; Okuno, Y.; Cavagnero, S. Sensitivity Enhancement in Solution NMR: Emerging Ideas and New Frontiers. J. Magn. Reson. 2014, 241, 18-31.

(34) Grage, S. L.; Xu, X.; Schmitt, M.; Wadhwani, P.; Ulrich, A. S. ${ }^{19}$ F-Labeling of Peptides Revealing Long-Range NMR Distances in Fluid Membranes. J. Phys. Chem. Lett. 2014, 5, 4256-4259.

(35) Pell, A. J.; Pintacuda, G.; Grey, C. P. Paramagnetic NMR in Solution and the Solid State. Prog. Nucl. Magn. Reson. Spectrosc. 2019, 111, 1-271.

(36) Kocman, V.; Di Mauro, G. M.; Veglia, G.; Ramamoorthy, A. Use of Paramagnetic Systems to Speed-up NMR Data Acquisition and for Structural and Dynamic Studies. Solid State Nucl. Magn. Reson. 2019, 102, 36-46.

(37) Parthasarathy, S.; Yoo, B.; McElheny, D.; Tay, W.; Ishii, Y. Capturing a Reactive State of Amyloid Aggregates: Nmr-Based Characterization of Copper-Bound Alzheimer Disease Amyloid $\beta$-Fibrils in a Redox Cycle. J. Biol. Chem. 2014, 289 (14), 9998-10010.

(38) Ashbrook, S. E.; Jaroniec, C. P. NMR Spectroscopy of Paramagnetic Solids. Solid State Nucl. Magn. Reson. 2019, 104, 101625.

(39) Mukhopadhyay, D.; Nadaud, P. S.; Shannon, M. D.; Jaroniec, C. P. Rapid Quantitative Measurements of Paramagnetic Relaxation Enhancements in $\mathrm{Cu}(\mathrm{II})$-Tagged Proteins by Proton-Detected Solid-State NMR Spectroscopy. J. Phys. Chem. Lett. 2017, 8 (23), 58715877.

(40) Perez, A.; Gaalswyk, K.; Jaroniec, C. P.; MacCallum, J. L. High Accuracy Protein Structures from Minimal Sparse Paramagnetic Solid-State NMR Restraints. Angew. Chemie - Int. Ed. 2019, $58(20), 6564-6568$.

(41) Theint, T.; Xia, Y.; Nadaud, P. S.; Mukhopadhyay, D.; Schwieters, C. D.; Surewicz, K.; Surewicz, W. K.; Jaroniec, C. P. Structural Studies of Amyloid Fibrils by Paramagnetic SolidState Nuclear Magnetic Resonance Spectroscopy. J. Am. Chem. Soc. 2018, 140 (41), 1316113166.

(42) Cai, S.; Seu, C.; Kovacs, Z.; Sherry, A. D.; Chen, Y. Sensitivity Enhancement of 
Multidimensional NMR Experiments by Paramagnetic Relaxation Effects. J. Am. Chem. Soc. 2006, 128, 13474-13478.

(43) Yamamoto, K.; Vivekanandan, S.; Ramamoorthy, A. Fast NMR Data Acquisition from Bicelles Containing a Membrane-Associated Peptide at Natural-Abundance. J. Phys. Chem. B 2011, 115 (43), 12448-12455.

(44) Bloch, F. Nuclear Induction. Phys. Rev. 1946, 70 (7), 460-474.

(45) Tesch, D. M.; Nevzorov, A. A. Sensitivity Enhancement and Contrasting Information Provided by Free Radicals in Oriented-Sample NMR of Bicelle-Reconstituted Membrane Proteins. $J$. Magn. Reson. 2014, 239, 9-15.

(46) Li, W.; Celinski, V. R.; Weber, J.; Kunkel, N.; Kohlmann, H. Homogeneity of Doping with Paramagnetic Ions by NMR. Phys. Chem. Chem. Phys 2016, 18, 9752.

(47) Lo, A. Y. H.; Sudarsan, V.; Sivakumar, S.; Van Veggel, F.; Schurko, R. W. Multinuclear SolidState NMR Spectroscopy of Doped Lanthanum Fluoride Nanoparticles. J. Am. Chem. Soc. 2007, 129 (15), 4687-4700.

(48) Nitsche, C.; Otting, G. Chapter 2: Intrinsic and Extrinsic Paramagnetic Probes. In New Developments in NMR; 2018; 42-84.

(49) Yagi, H.; Banerjee, D.; Graham, B.; Huber, T.; Goldfarb, D.; Otting, G. Gadolinium Tagging for High-Precision Measurements of $6 \mathrm{Nm}$ Distances in Protein Assemblies by EPR. J. Am. Chem. Soc 2011, 133, 10418-10421.

(50) Potapov, A.; Yagi, H.; Huber, T.; Jergic, S.; Dixon, N. E.; Otting, G.; Goldfarb, D. NanometerScale Distance Measurements in Proteins Using $\mathrm{Gd}^{3+}$ Spin Labeling. J. Am. Chem. Soc. 2010, 132, 9040-9048.

(51) Luchinat, C.; Parigi, G.; Ravera, E. Paramagnetism in Experimental Biomolecular NMR; 2018.

(52) Bertini, I.; Luchinat, C.; Parigi, G.; Ravera, E. Lanthanoids and Actinoids: Shift and Relaxation. In Solution NMR of Paramagnetic Molecules; Elsevier, 2016; 255-276.

(53) Otting, G. Prospects for Lanthanides in Structural Biology by NMR. J. Biomol. NMR 2008, 42 (1), 1-9.

(54) Bertini, I.; Luchinat, C.; Parigi, G.; Ravera, E. Chapter 2 - The Hyperfine Shift. In NMR of Paramagnetic Molecules; Bertini, I., Luchinat, C., Parigi, G., Ravera, E. B. T.-N. M. R. of P. 
M. (Second E., Eds.; Elsevier: Boston, 2017; pp 25-60.

(55) Strickland, M.; Catazaro, J.; Rajasekaran, R.; Strub, M. P.; O'Hern, C.; Bermejo, G. A.; Summers, M. F.; Marchant, J.; Tjandra, N. Long-Range RNA Structural Information via a Paramagnetically Tagged Reporter Protein. J. Am. Chem. Soc. 2019, 141 (4), 1430-1434.

(56) Kooshapur, H.; Schwieters, C. D.; Tjandra, N. Conformational Ensemble of Disordered Proteins Probed by Solvent Paramagnetic Relaxation Enhancement (SPRE). Angew. Chemie Int. Ed. 2018, 57 (41), 13519-13522.

(57) Miao, Q.; Liu, W. M.; Kock, T.; Blok, A.; Timmer, M.; Overhand, M.; Ubbink, M. A DoubleArmed, Hydrophilic Transition Metal Complex as a Paramagnetic NMR Probe. Angew. Chemie - Int. Ed. 2019, 58 (37), 13093-13100.

(58) Camacho-Zarco, A. R.; Munari, F.; Wegstroth, M.; Liu, W. M.; Ubbink, M.; Becker, S.; Zweckstetter, M. Multiple Paramagnetic Effects through a Tagged Reporter Protein. Angew. Chemie - Int. Ed. 2015, 54 (1), 336-339.

(59) Liu, W.-M.; Skinner, S. P.; Timmer, M.; Blok, A.; Hass, M. A. S.; Filippov, D. V.; Overhand, M.; Ubbink, M. A Two-Armed Lanthanoid-Chelating Paramagnetic NMR Probe Linked to Proteins via Thioether Linkages. Chem. - A Eur. J. 2014, 20 (21), 6256-6258.

(60) Nitsche, C.; Otting, G. Pseudocontact Shifts in Biomolecular NMR Using Paramagnetic Metal Tags. Prog. Nucl. Magn. Reson. Spectrosc. 2017, 98-99, 20-49.

(61) Orton, H. W.; Otting, G. Accurate Electron-Nucleus Distances from Paramagnetic Relaxation Enhancements. J. Am. Chem. Soc. 2018, 140 (24), 7688-7697.

(62) Pilla, K. B.; Otting, G.; Huber, T. 3D Computational Modeling of Proteins Using Sparse Paramagnetic NMR Data. In Bioinformatics: Volume II: Structure, Function, and Applications; Keith, J. M., Ed.; Springer New York: New York, NY, 2017; pp 3-21.

(63) Hardin, N. Z.; Kocman, V.; Di Mauro, G. M.; Ravula, T.; Ramamoorthy, A. Metal-Chelated Polymer Nanodiscs for NMR Studies. Angew. Chemie - Int. Ed. 2019, 58 (48), 17246-17250.

(64) Ravula, T.; Ramadugu, S. K.; Di Mauro, G. M.; Ramamoorthy, A. Bioinspired, Size-Tunable Self-Assembly of Polymer-Lipid Bilayer Nanodiscs. Angew. Chemie Int. Ed. 2017, 56, 1146611470.

(65) Ravula, T.; Ramamoorthy, A. Magnetic Alignment of Polymer Macro-Nanodiscs Enables Residual-Dipolar-Coupling-Based High-Resolution Structural Studies by NMR Spectroscopy. 
Angew. Chemie - Int. Ed. 2019, 58 (42), 14925-14928.

(66) Mahawaththa, M. C.; Lee, M. D.; Giannoulis, A.; Adams, L. A.; Feintuch, A.; Swarbrick, J. D.; Graham, B.; Nitsche, C.; Goldfarb, D.; Otting, G. Small Neutral Gd(III) Tags for Distance Measurements in Proteins by Double Electron-Electron Resonance Experiments. Phys. Chem. Chem. Phys. 2018, 20 (36), 23535-23545.

(67) Gamble Jarvi, A.; Cunningham, T. F.; Saxena, S. Efficient Localization of a Native Metal Ion within a Protein by $\mathrm{Cu}^{2+}$-Based EPR Distance Measurements. Phys. Chem. Chem. Phys. 2019, $21(20), 10238-10243$.

(68) Corsi, D. M.; Platas-Iglesias, C.; Van Bekkum, H.; Peters, J. A. Determination of Paramagnetic Lanthanide(III) Concentrations from Bulk Magnetic Susceptibility Shifts in NMR Spectra. Magn. Reson. Chem. 2001, 39 (11), 723-726.

(69) Bertmer, M. Paramagnetic Solid-State NMR of Materials. Solid State Nucl. Magn. Reson. 2017, 81, 1-7.

(70) Bertini, I.; Luchinat, C.; Parigi, G. Magnetic Susceptibility in Paramagnetic NMR. Prog. Nucl. Magn. Reson. Spectrosc. 2002, 40 (3), 249-273.

(71) Pintacuda, G.; Vaara, J.; Bertini, I.; Park, M.; Banci, L.; Bertini, I.; Bertini, I.; Bertini, I.; Luchinat, C. NMR of Paramagnetic Molecules in Biological Systems. 2001, 1-2.

(72) Suturina, E. A.; Mason, K.; Geraldes, C. F. G. C.; Chilton, N. F.; Parker, D.; Kuprov, I. Lanthanide-Induced Relaxation Anisotropy. Phys. Chem. Chem. Phys. 2018, 20 (26), 1767617686.

(73) Bertini, I.; Janik, M. B. L.; Lee, Y. M.; Luchinat, C.; Rosato, A. Magnetic Susceptibility Tensor Anisotropies for a Lanthanide Ion Series in a Fixed Protein Matrix. J. Am. Chem. Soc. 2001, $123(18), 4181-4188$.

(74) Ravula, T.; Ramadugu, S. K.; Di Mauro, G. M.; Ramamoorthy, A. Bioinspired, Size-Tunable Self-Assembly of Polymer-Lipid Bilayer Nanodiscs. Angew. Chemie Int. Ed. 2017, 56, $11466-11470$.

(75) Kumar, K.; Chang, C. A.; Francesconi, L. C.; Dischino, D. D.; Malley, M. F.; Gougoutas, J. Z.; Tweedle, M. F. Synthesis, Stability, and Structure of Gadolinium(III) and Yttrium(III) Macrocyclic Poly(Amino Carboxylates). Inorg. Chem. 1994, 33 (16), 3567-3575.

(76) Karraker, D. G. Coordination of Trivalent Lanthanide Ions. J. Chem. Eduction 1970, 47 (6), 
424-430.

(77) Dvinskikh, S. V; Zimmermann, H.; Maliniak, A.; Sandström, D. Measurements of Motionally Averaged Heteronuclear Dipolar Couplings in MAS NMR Using R-Type Recoupling. $J$. Magn. Reson. 2004, 168 (2), 194-201.

(78) Kurzen, H.; Bovigny, L.; Bulloni, C.; Daul, C. Electronic Structure and Magnetic Properties of Lanthanide 3+ Cations. Chem. Phys. Lett. 2013, 574, 129-132.

(79) Bertini. Appendix I NMR Properties of Nuclei. NMR Paramagn. Mol. 2017, 457-460.

(80) Bertini, I.; Luchinat, C.; Parigi, G.; Pierattelli, R. NMR Spectroscopy of Paramagnetic Metalloproteins. ChemBioChem 2005, 6 (9), 1536-1549.

(81) Yamamoto, K.; Xu, J.; Kawulka, K. E.; Vederas, J. C.; Ramamoorthy, A. Use of a CopperChelated Lipid Speeds Up NMR Measurements from Membrane Proteins. J. Am. Chem. Soc. 2010, 132 (20), 6929-6931.

(82) Bertini, I.; Luchinat, C.; Parigi, G.; Ravera, E. Chapter 8 - Lanthanoids and Actinoids: Shift and Relaxation. In NMR of Paramagnetic Molecules; Bertini, I., Luchinat, C., Parigi, G., Ravera, E. B. T.-N. M. R. of P. M. (Second E., Eds.; Elsevier: Boston, 2017; 255-276. 


\section{Chapter 4}

Benchmarks of SMA-Copolymer Derivatives and Nanodiscs Integrity

The content for this chapter is partially included in the following research article:

Di Mauro Giacomo M., La Rosa Carmelo, Condorelli Marcello, Ramamoorthy Ayyalusamy, Benchmarks of SMA-copolymer derivatives and nanodiscs integrity, (Manuscript in preparation) 


\subsection{Introduction}

Membrane proteins, essential components of any cellular membrane, are involved in many crucial cellular functions required for life and represent $\sim 60 \%$ of all drug targets ${ }^{1}$. However, despite the recent success in obtaining high-resolution structures ${ }^{2-5}$, our current understanding of cellular membrane biology and the development of modern drugs is hindered by the lack of structural information tied to the challenges in the extraction, isolation, and purification of functioning membrane proteins from their native cellular environment ${ }^{6-13}$. Poly(styrene-co-maleic acid), or SMA, is an amphipathic copolymer used to directly solubilize cell membranes and stabilize membrane proteins in native-like discoidal copolymer-lipid nanoparticles, constituted by a lipid bilayer patch wrapped in a belt of amphipathic copolymer chains ${ }^{8-10,14-20}$. Acting, de facto, as a «macromolecular detergent,» among its advantages, SMA copolymer allows membrane proteins to retain lipids that are useful for both structural and functional purposes ${ }^{2-4,10,21-27}$, showing no preferences in the extraction ${ }^{28}$.

SMA copolymer, however, is a generic denomination that identifies a variety of different formulations (SMAs); in fact, it can be readily customized ${ }^{29-34}$. For example, by varying the molecular weight or the styrene-to-maleic acid ratio, it is possible to fine-tune the amphipathic properties and so as the ability of membrane solubilization ${ }^{35}$. Additionally, SMA copolymers can be further functionalized, expanding its range of applications ${ }^{11,17,19,36-40}$. Each formulation has both advantages and limitations that affect its applicability ${ }^{41}$. Indeed, the adoption of the suitable formulation is mostly connected to convenience, and a trial-and-error process since each studied system requires adapted protocols ${ }^{42}$.

Due to the amphipathic nature, SMAs exist mainly as individual copolymer chains in dilute solutions, while at high concentrations form a plethora of intra- and inter-chains copolymer micelles ${ }^{43}$. Such a variety of results, modulated by the balance of hydrophobic effect and electrostatic repulsions, is affected by the dispersity $(\mathrm{Ð})^{35}$. Many parameters, such as the molecular weight (MW), the styreneto-maleic acid ratio (x:y), and the $Đ$, are currently used to assess SMA formulations. Additionally, as polymeric «detergent,» SMAs can be evaluated using parameters such as the critical micellar concentration (c.m.c.) $)^{44}$.

Despite its definition, c.m.c. does not correspond to a single well-defined value. Still, it coincides with a range of concentrations, and it involves dynamic, association-dissociation equilibrium ${ }^{43,45}$. In the case of amphiphilic copolymers, their dispersity complicates the scenario. Indeed, the presence of chains of various molecular weights ${ }^{29}$ leads to a distribution of aggregates of different sizes, both intra- and inter-chains. There have been many attempts to decrease the copolymers' intrinsic dispersity 
by purification steps or finding alternative synthetic paths, but studies suggest that SMA's solubilization efficiency is connected to the variation of molecular weights ${ }^{33,43}$.

Copolymer-lipid nanodiscs are functional hybrid materials that arise from the synergistic interactions between two self-assembling materials, copolymers and phospholipids. For this reason, it is misleading to refer to both copolymer and phospholipids as individual entities. Indeed, once mixed, copolymers interact with phospholipids forming either nanodiscs or other randomly unstructured aggregates.

The necessity of benchmarking the performances of existing copolymers, assessing crucial parameters for the successful extraction and stabilization of membrane proteins is compulsory for advancing the field. To facilitate the choice among the copolymer formulations commercially available, assist the design of new alternatives, and shed light on copolymer-lipid nanodiscs' behavior, herein we compare a set of anionic SMAs. XIRAN ${ }^{\circledR}$ SL25010 P, SL30010 P, SL40005 P formulations (gift from Polyscience ${ }^{\circledR}$ (Geleen, Netherlands)) were chosen because of their wide diffusion and success rate and compared to SMA-EA, a copolymer developed in our laboratory ${ }^{36}$. Each copolymer exhibits different molecular weight, hydrophobic-hydrophilic ratio, and charge density, covering a wide range of features.

\subsection{Materials and Methods}

\subsubsection{Reagents and Materials}

1,2-dimyristoyl-sn-glycero-3-phosphocholine (DMPC) was purchased from Avanti ${ }^{\circledR}$ Polar Lipids, Inc. (Alabaster, Alabama). Hydrochloric Acid $(\mathrm{HCl})$, Sodium Hydroxide $(\mathrm{NaOH})$, Ammonium Acetate $\left(\mathrm{C}_{2} \mathrm{H}_{7} \mathrm{NO}_{2}\right)$, Potassium phosphate monobasic $\left(\mathrm{KH}_{2} \mathrm{PO}_{4}\right)$, Potassium phosphate dibasic $\left(\mathrm{K}_{2} \mathrm{HPO}_{4}\right)$, Pyrene $\left(\mathrm{C}_{16} \mathrm{H}_{10}\right)$, Sodium Cholate hydrate $\left(\mathrm{C}_{24} \mathrm{H}_{39} \mathrm{NaO}_{5}\right)$ were purchased from SigmaAldrich $^{\circledR}$ (St. Louis, Missouri). SMA-EA was synthesized, purified, and characterized accordingly to the procedure described in the literature ${ }^{36}$. XIRAN ${ }^{\circledR}$ SL25010 P, XIRAN ${ }^{\circledR}$ SL30010 P, XIRAN ${ }^{\circledR}$ SL40005 P were kindly gifted by Polyscience ${ }^{\circledR}$ (Geleen, Netherlands).

\subsubsection{Methods}

a) Copolymer-lipid nanodiscs preparation. A $20 \mathrm{mg} / \mathrm{mL}$ stock solution of each copolymer was prepared by weighing the copolymer in powder and dissolving it upon addition of $0.1 \mathrm{M} \mathrm{NaOH}$. After complete solubilization, the solution was neutralized by adding $1 \mathrm{M} \mathrm{HCl}$ dropwise, 
reaching a final $\mathrm{pH}$ 7.00. Copolymer-based lipid nanodiscs were obtained, mixing DMPC and copolymers according to a 1:1 weight ratio $(\mathrm{w} / \mathrm{w})$. Each sample was then diluted to the desired final volume and incubated overnight at room temperature. SMA-EA, SL25010 P, SL30010 P copolymer solution samples for the pyrene assay were prepared at an initial concentration of 2 $\mathrm{mg} / \mathrm{mL}$. For the SL40005 P solution, due to this copolymer's high hydrophilicity, the starting concentration was set to $25 \mathrm{mg} / \mathrm{mL}$. Copolymer-lipid nanodiscs were purified by size exclusion chromatography. For each copolymer formulation, $10 \mathrm{mg}$ of lipids and $10 \mathrm{mg}$ of copolymers were mixed, as explained previously, using $10 \mathrm{mM}$ phosphate buffer at $\mathrm{pH} 7.2$ and eluted at room temperature. All the aliquots associated with the nanodiscs' peak were collected and mixed. This homogeneous solution was then divided into four aliquots of equal volume. Three of them were then used to perform the pyrene assay, while one was used for quantification using NMR experiments.

b) Preparation of the pyrene-buffer stock solution. A stock solution was prepared by solubilizing a known amount of pyrene in ethanol $(95 \% \mathrm{v} / \mathrm{v})$. A $1 \mu \mathrm{M}$ water-based pyrene solution was then made from its dilution with $10 \mathrm{mM}$ ammonium acetate buffer $\left(\mathrm{pH} 7\right.$ at $\left.25^{\circ} \mathrm{C}\right)$. The study of purified nanodiscs was performed using $10 \mathrm{mM}$ potassium phosphate buffer $\left(\mathrm{pH} 7\right.$ at $25^{\circ} \mathrm{C}$ ) to avoid spectral interferences with ${ }^{1} \mathrm{H}$ NMR. The ethanol content in the experimental solution was considered negligible and, as reported in the literature, was found not to affect the spectral and self-aggregation behavior of amphiphiles.

c) Pyrene fluorescence study. Using pyrene as the fluorescent probe, fluorescence measurements were taken in a FluoroMax $-4{ }^{\circledR}$ Spectrofluorometer from Horiba Scientific ${ }^{\circledR}$, using a $4 \mathrm{~mL}$ quartz cuvette with $1 \mathrm{~cm}$ optical path. Excitation was done at $331.5 \mathrm{~nm}$, and emissions were recorded in the 340-350 $\mathrm{nm}$ wavelength range. The slit widths for both excitation and emission were fixed at $0.4 \mathrm{~nm}$. A $2 \mathrm{mg} / \mathrm{mL}$ solution of amphipathic species (copolymers and copolymer-nanodiscs), dissolved in buffer $\left(\mathrm{pH} 7\right.$ at $25^{\circ} \mathrm{C}$ ) and $1 \mu \mathrm{M}$ pyrene, was progressively diluted by removing 500 $\mu \mathrm{L}$ and adding $500 \mu \mathrm{L}$ of a fresh pyrene-containing buffer. Thus, the pyrene concentration is constant through the dilution of the amphipathic species. The scan time was fixed at $0.8 \mathrm{~s}$ per scan. All measurements were thermostatically controlled at $25.0 \pm 0.1{ }^{\circ} \mathrm{C}$ using a Quantum Northwest TC 1 temperature controller ${ }^{46}$. The ratio between the intensity of the fluorescence peaks $I_{1}$ and $I_{3}$ is plotted against the decimal logarithm of the considered amphipathic species' concentration. Spectra, experimental data, and fitting are shown in Figures 4.1-4.4, 4.13,4.14 and 4.16. Numerical values are given in Tables 4.1 and 4.2 . 


\section{a) SMA-EAcopolymer}

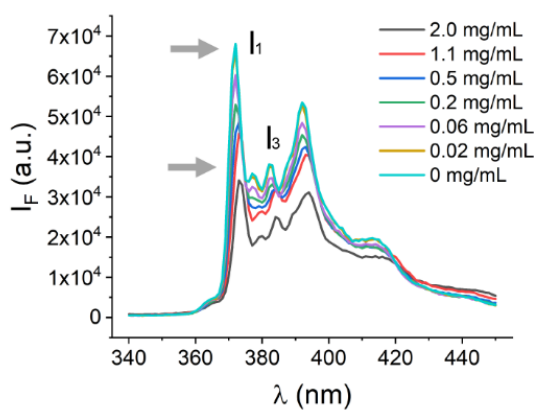

b) SL25010 P copolymer

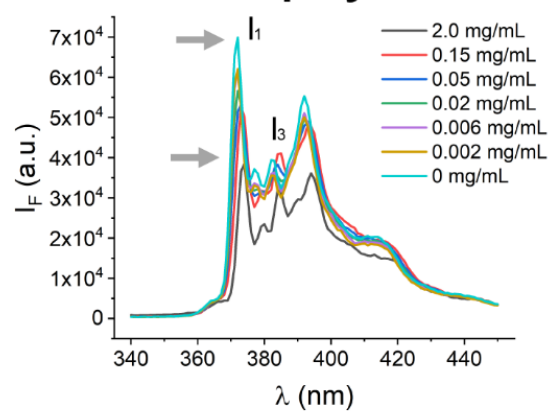

c) SL30010 P copolymer

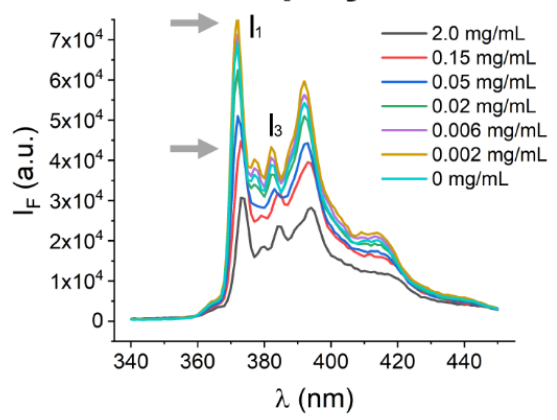

\section{d) SL40005 P copolymer}

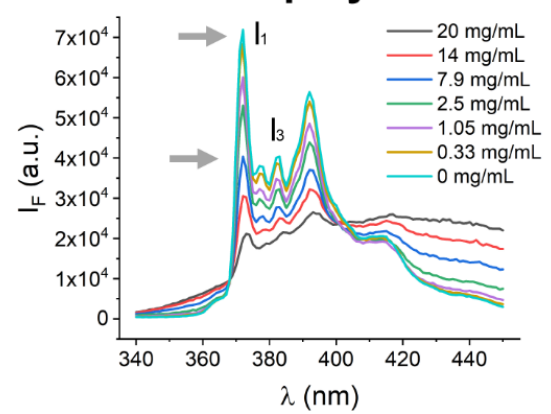

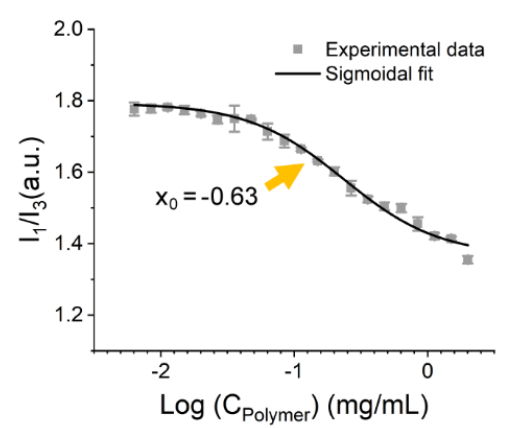
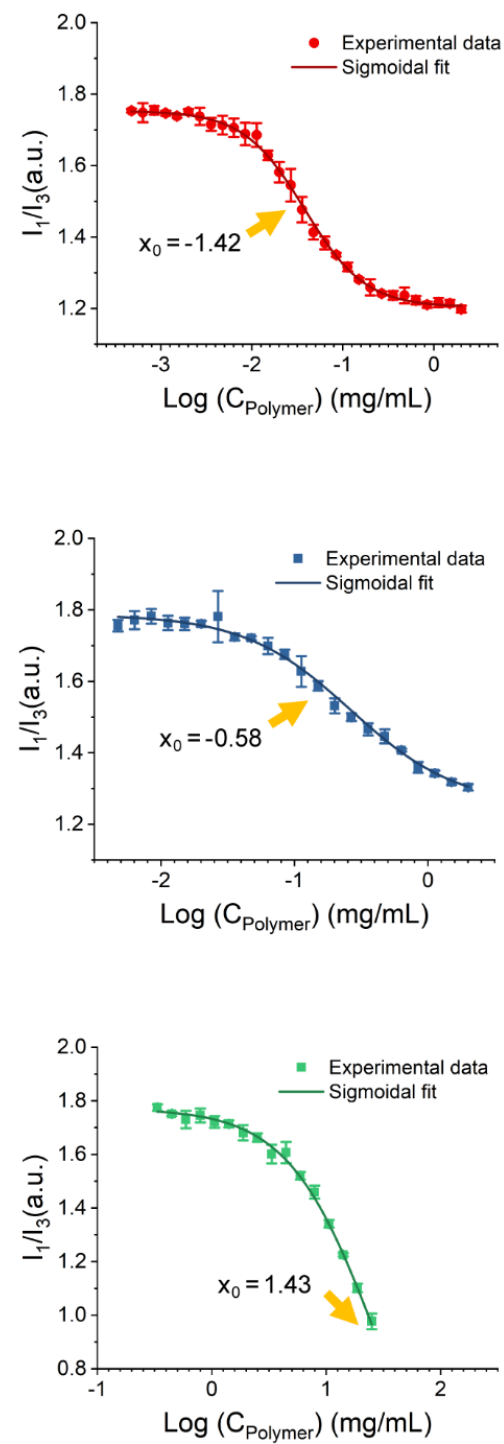

Figure 4.1. Pyrene essay on copolymer solutions. Panels on the left show a selection of pyrene fluorescent spectra in the presence of various concentrations of copolymers. Gray arrows highlight the variation in the fluorescence intensity of the peaks $I_{1}$ and $I_{3}$ for varying the concentration of copolymer. The change is consistent among the whole spectrum. Panels on the right show the $\mathrm{I}_{1} / \mathrm{I}_{3}$ average of three independent experiments against the decimal logarithm of the polymer concentration (in $\mathrm{mg} / \mathrm{mL}$ ). Error bars correspond to the standard deviation among them. The yellow arrow points at the flex $\left(\mathrm{x}_{0}\right)$ of each curve obtained using a sigmoidal fitting. 


\section{a) Unpurified DMPC:SMA-EA $(1: 1 \mathrm{w} / \mathrm{w})$}
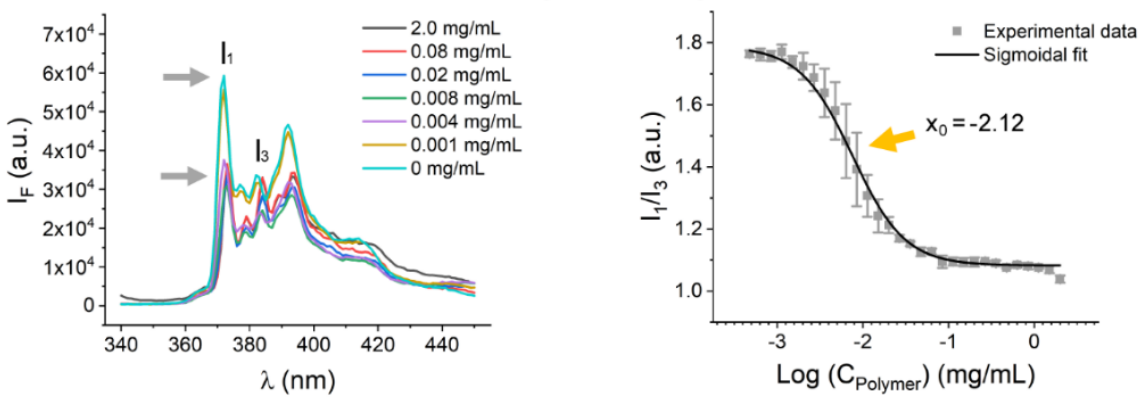

b) Unpurified DMPC:SL25010 P (1:1 w/w)
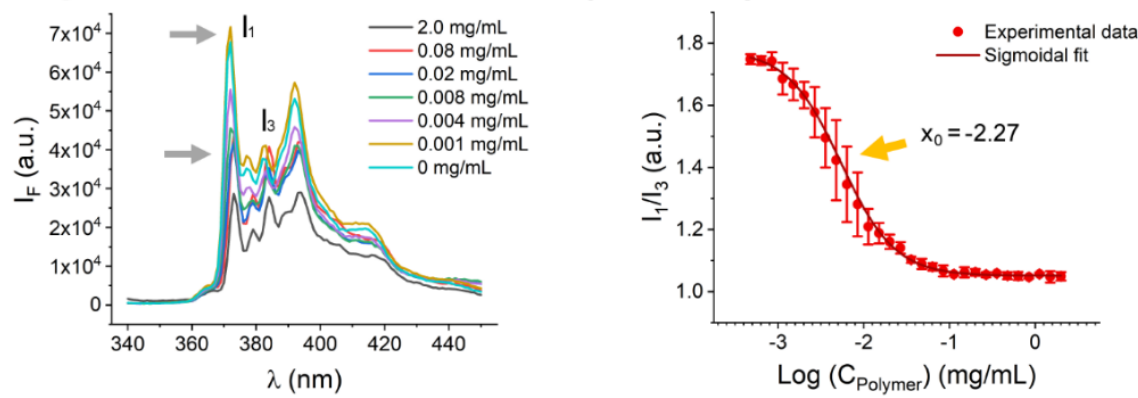

c) Unpurified DMPC:SL30010 P (1:1 w/w)
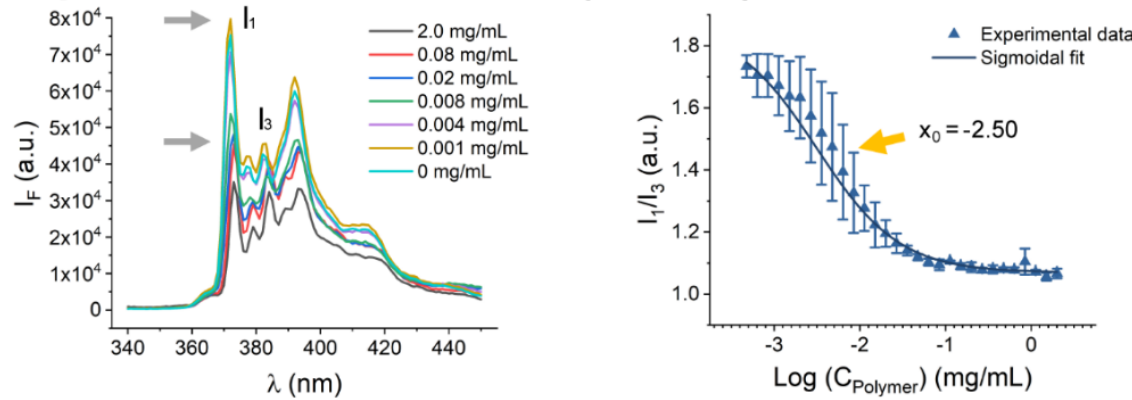

d) Unpurified DMPC:SL40005 P (1:1 w/w)
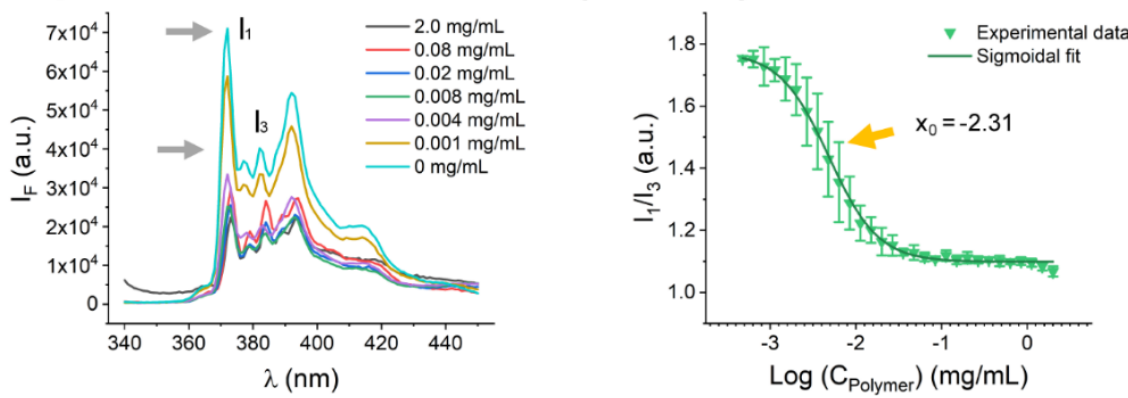

Figure 4.2. Pyrene essay on unpurified copolymer-lipid nanodiscs solutions as a function of the copolymer concentration $(\mathbf{m g} / \mathbf{m L})$. Panels on the left show a selection of pyrene fluorescent spectra in the presence of various concentrations of copolymers. Gray arrows highlight the variation in the fluorescence intensity of the peaks $I_{1}$ and $I_{3}$ for varying the concentration of copolymer. The change is consistent among the whole spectrum. Panels on the right show the $\mathrm{I}_{1} / \mathrm{I}_{3}$ average of three independent experiments against the decimal logarithm of the polymer concentration (in $\mathrm{mg} / \mathrm{mL}$ ). Error bars correspond to the standard deviation among them. The yellow arrow points at the flex $\left(\mathrm{x}_{0}\right)$ of each curve obtained using a sigmoidal fitting. 
a) Unpurified DMPC:SMA-EA (1:1 w/w)
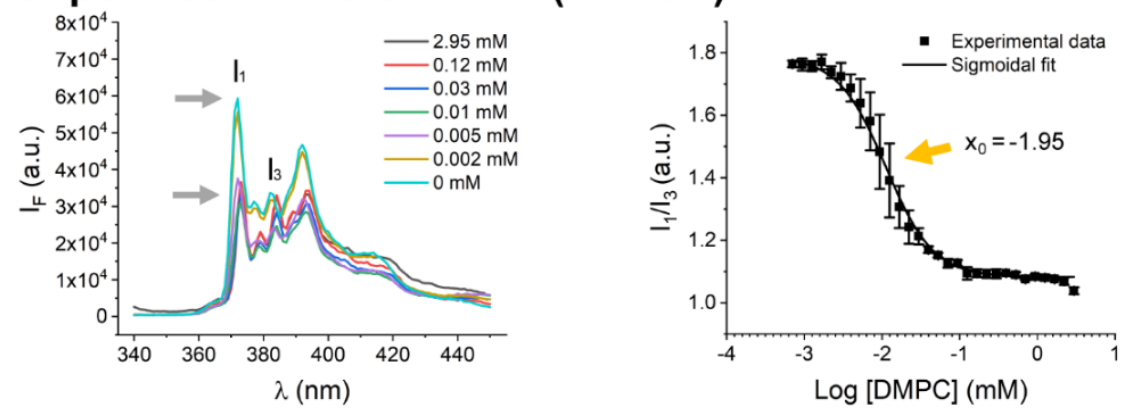

b) Unpurified DMPC:SL25010 P (1:1 w/w)
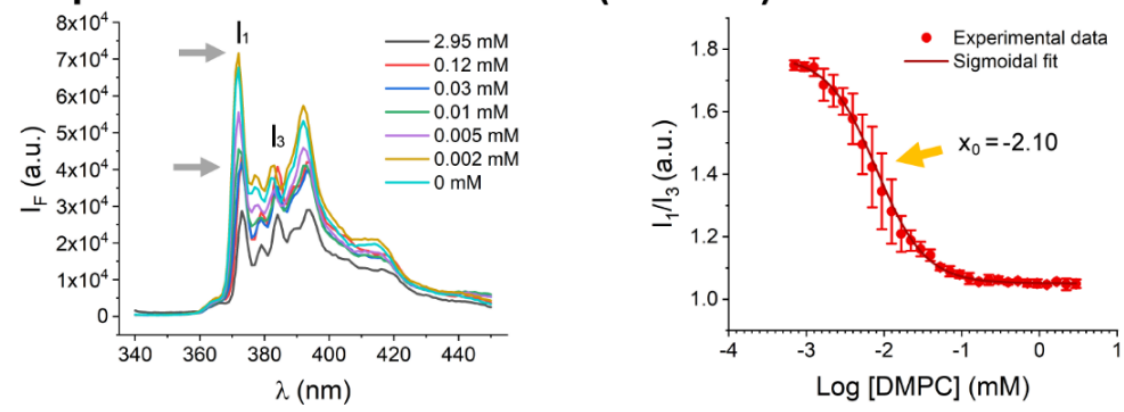

c) Unpurified DMPC:SL30010 P (1:1 w/w)
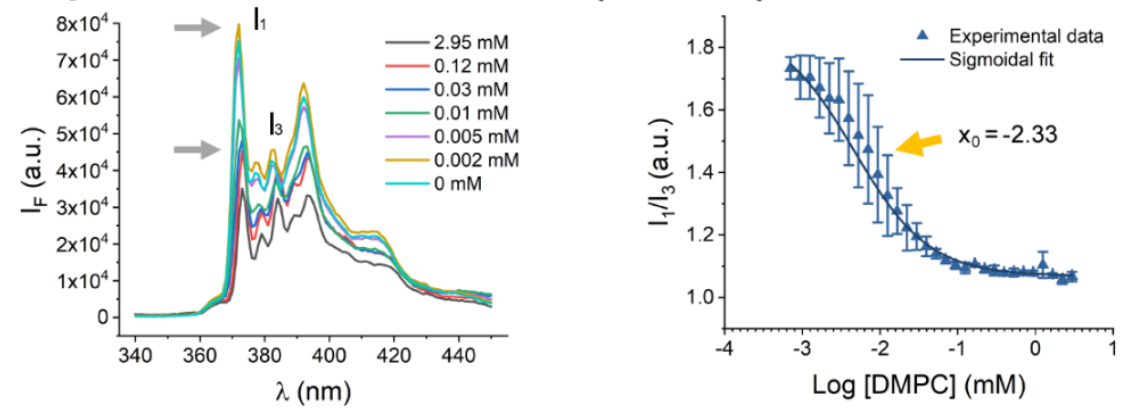

d) Unpurified DMPC:SL40005 P (1:1 w/w)
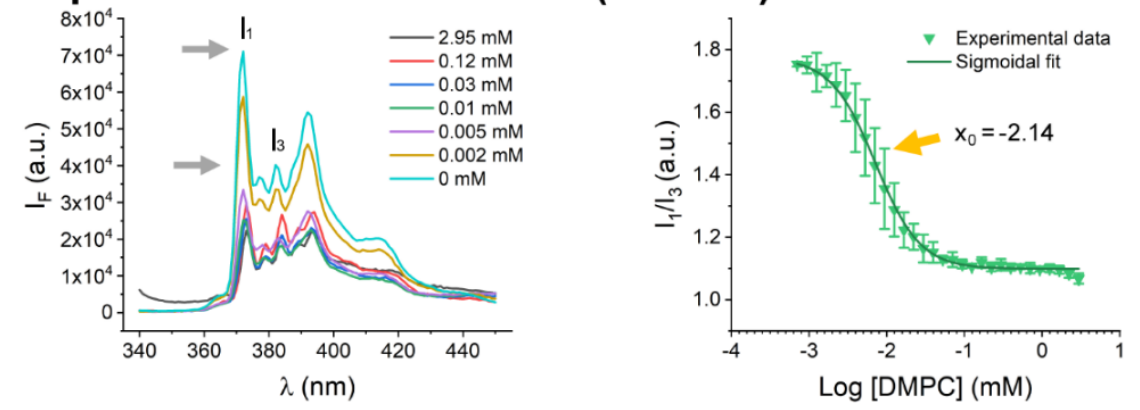

Figure 4.3. Pyrene essay on unpurified copolymer-lipid nanodises solutions as a function of phospholipids concentration (mM). Panels on the left show a selection of pyrene fluorescent spectra in the presence of various concentrations of copolymers. Gray arrows highlight the variation in the fluorescence intensity of the peaks $I_{1}$ and $I_{3}$ for varying the concentration of copolymer. The change is consistent among the whole spectrum. Panels on the right show the $\mathrm{I}_{1} / \mathrm{I}_{3}$ average of three independent experiments against the decimal logarithm of the lipid concentration (in $\mathrm{mM}$ ). Error bars correspond to the standard deviation among them. The yellow arrow points at the flex $\left(\mathrm{x}_{0}\right)$ of each curve obtained using a sigmoidal fitting. 


\section{a) SEC-Purified DMPC:SMA-EA(1:1 w/w)}
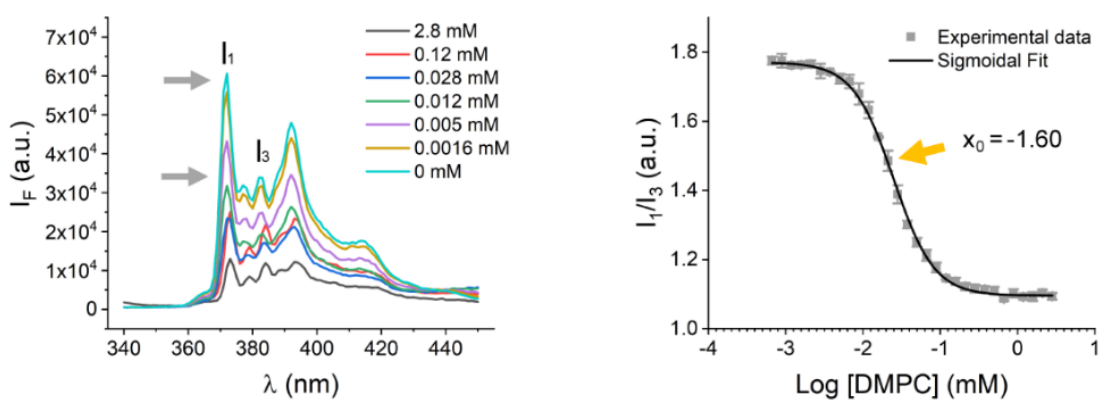

b) SEC-Purified DMPC:SL25010 P (1:1 w/w)
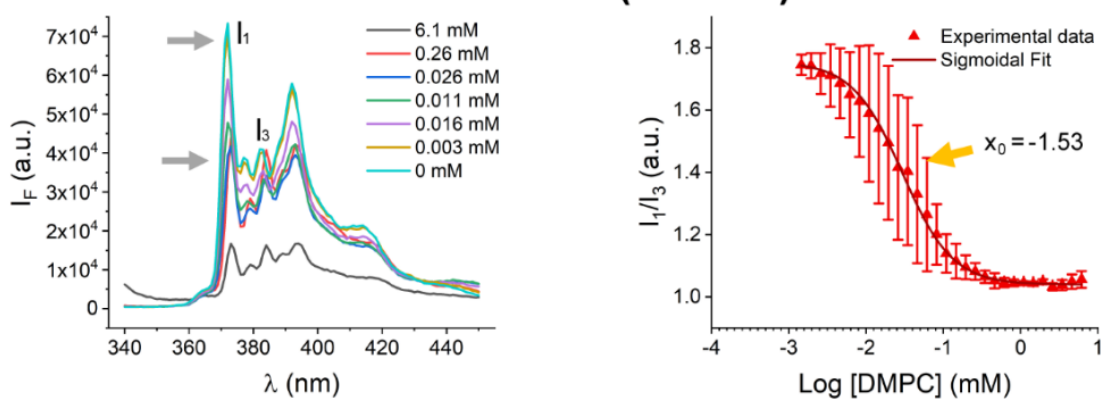

c) SEC-Purified DMPC:SL30010 P (1:1 w/w)
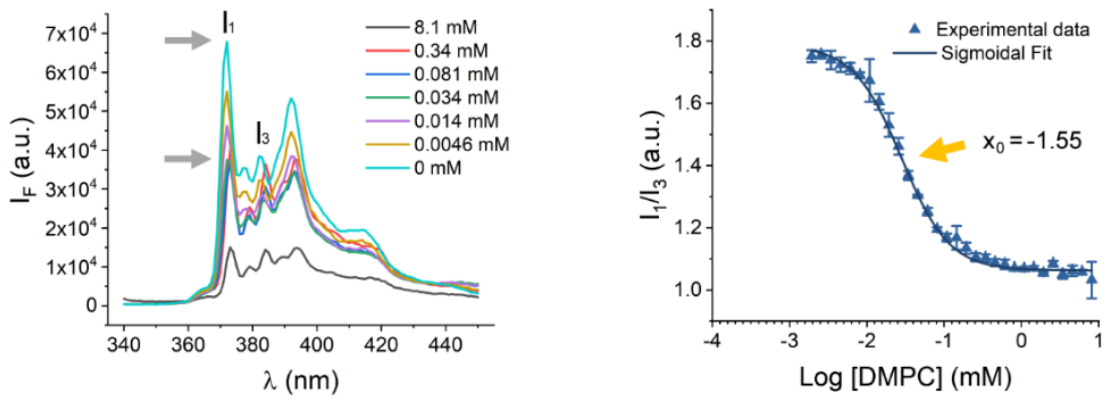

d) SEC-Purified DMPC:SL40005 P (1:1 w/w)
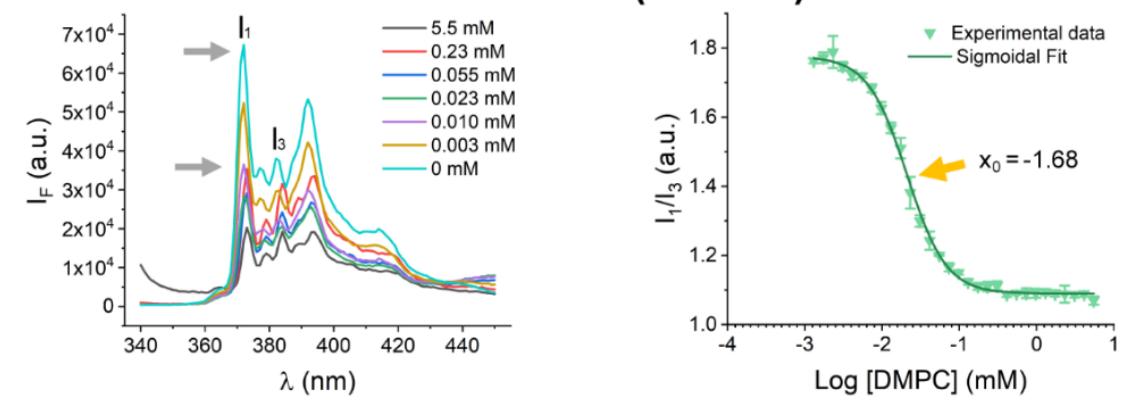

Figure 4.4. Pyrene essay on SEC-purified copolymer-lipid nanodiscs solutions as a function of phospholipids concentration $(\mathbf{m M})$. Panels on the left show a selection of pyrene fluorescent spectra in the presence of various concentrations of copolymers. Gray arrows highlight the variation in the fluorescence intensity of the peaks $I_{1}$ and $I_{3}$ for varying the concentration of copolymer. The change is consistent among the whole spectrum. Panels on the right show the $I_{1} / I_{3}$ average of three independent experiments against the decimal logarithm of the lipid concentration (in $\mathrm{mM}$ ). Error bars correspond to the standard deviation among them. The yellow arrow points at the flex $\left(\mathrm{x}_{0}\right)$ of each curve obtained using a sigmoidal fitting. 
d) Size Exclusion Chromatography (SEC). Copolymer-lipid nanodiscs were purified using a selfpacked Sephadex 200 16/600 column operated on a GE Healthcare ${ }^{\circledR}$ AKTA purifier. Samples were eluted at room temperature and a buffer flow rate of $1 \mathrm{~mL} / \mathrm{min}$. The buffer used was $10 \mathrm{mM}$ potassium phosphate buffer, $\mathrm{pH} 7$ at $25^{\circ} \mathrm{C}$. The elution was monitored using a UV-detector at $\lambda=254 \mathrm{~nm}$. Chromatograms are shown below in Figure 4.5.

a)

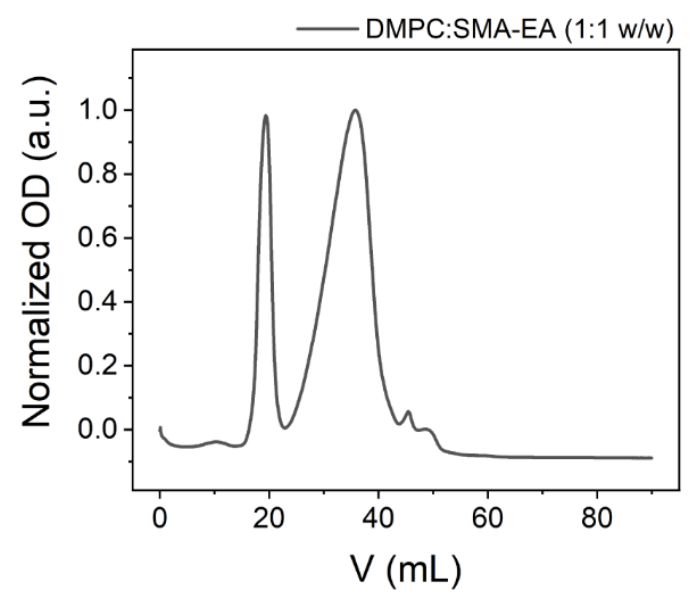

c)

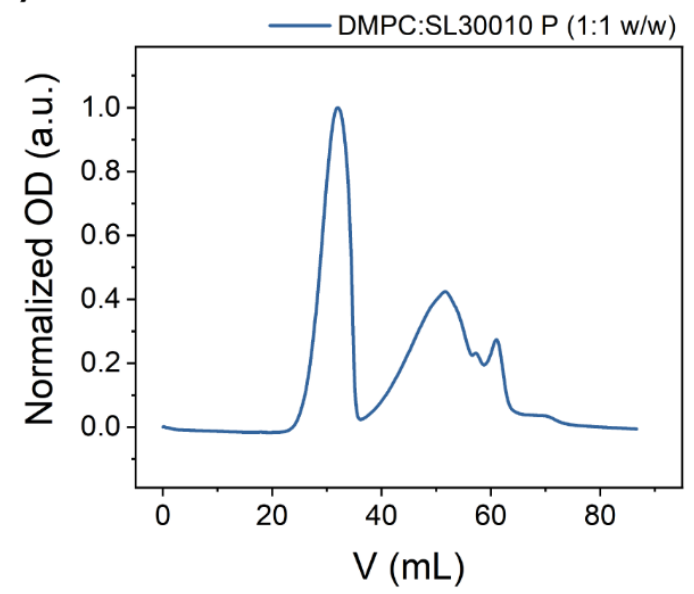

b)

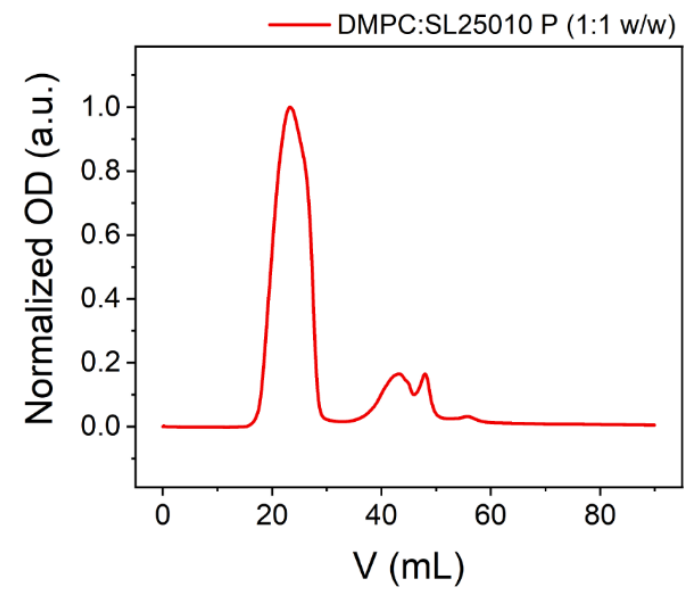

d)

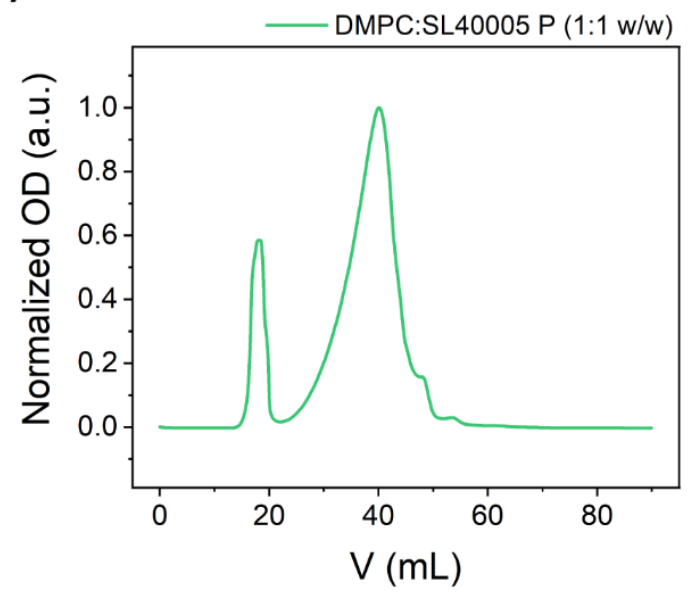

Figure 4.5. Size Exclusion Chromatograms of 1:1 w/w copolymer-DMPC nanodiscs. For each sample, the first peak is associated with the copolymer-lipid nanodiscs, while the second peak is associated with the micellar species formed by each copolymer in solution. 
e) NMR Spectroscopy. Solution ${ }^{1} \mathrm{H}-\mathrm{NMR}$ experiments were performed using a $500 \mathrm{MHz}$ Bruker Avance III HD NMR spectrometer. NMR samples were prepared according to the procedure described in the «Copolymer-lipid nanodiscs preparation» paragraph but using $10 \mathrm{mM}$ sodium phosphate buffer, $\mathrm{pH} 7.2$, to avoid spectral interferences. All samples analyzed through NMR spectroscopy were lyophilized for 24 hours before resuspension in $600 \mu \mathrm{L}$ of $10 \mathrm{mM}$ sodium cholate in $\mathrm{D}_{2} \mathrm{O}$ and then transferred to $4 \mathrm{~mm}$ Norrell ${ }^{\circledR}$ Sample Vault Series ${ }^{\mathrm{TM}}$ glass tubes and placed in a commercial $5 \mathrm{~mm}$ triple-resonance ${ }^{1} \mathrm{H} /{ }^{19} \mathrm{~F} /{ }^{13} \mathrm{C}$ Bruker round-coil TXITM $500 \mathrm{SB}$ probe. ${ }^{1} \mathrm{H}$ NMR spectra were recorded by collecting 32 scans with a spectral width of 25 ppm using a recycle delay of $1 \mathrm{~s}$. The ${ }^{1} \mathrm{H}$ transmitter frequency offset was set at the water proton peak (4.7 ppm). The experiments were performed at $35^{\circ} \mathrm{C}$ to maximize lipid peaks' sharpness and ensure better integration. The concentration of each sample was measured using sodium cholate as an internal standard. ${ }^{1} \mathrm{H}$ NMR spectra and area integration for peaks are shown in Figures 4.64.10. Data have been processed using Bruker Topspin ${ }^{\mathrm{TM}} 3.2$ and 4.0.7.

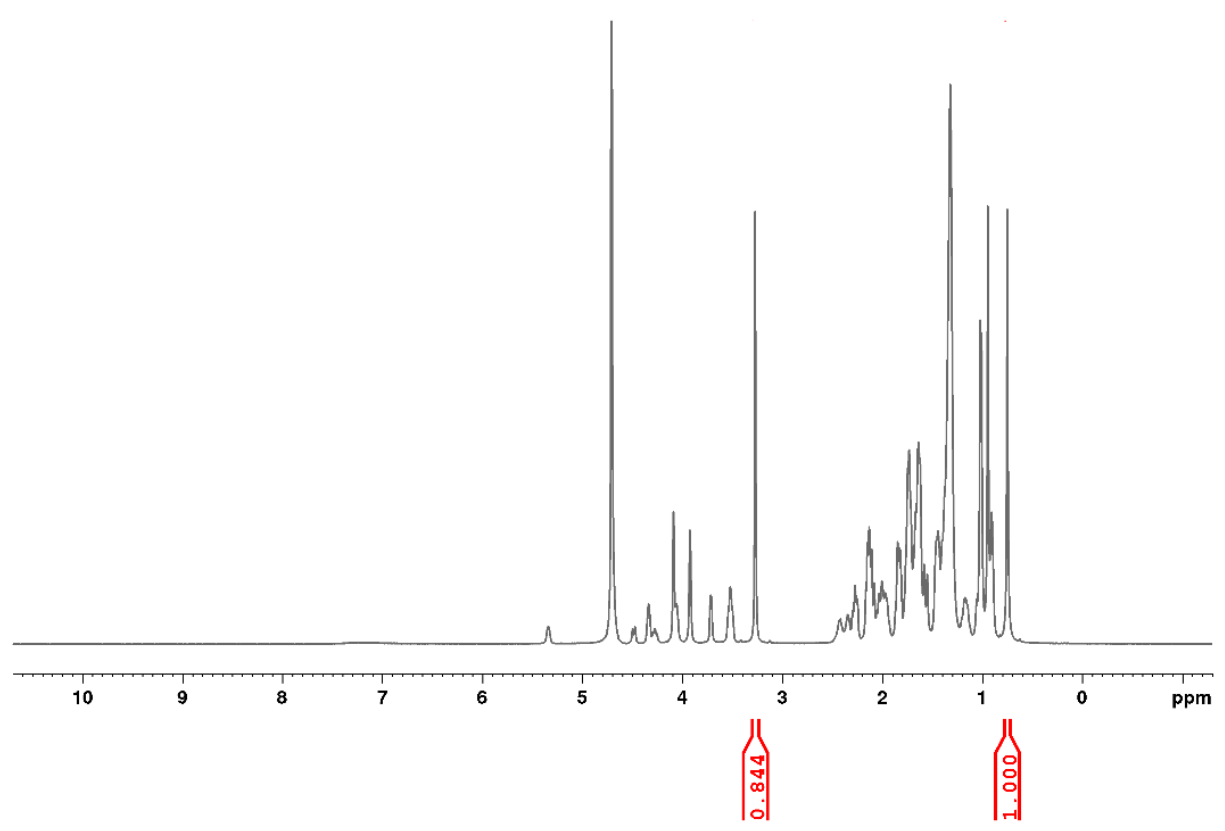

Figure 4.6. ${ }^{1}$ H-NMR spectra of SMA-EA:DMPC 1:1 (w/w) nanodiscs. Samples are recorded in the presence of 10 $\mathrm{mM}$ sodium cholate. To assess the molar concentration, the peak associated with quaternary ammonium protons from DMPC $(\sim 3.3 \mathrm{ppm})$ is integrated vs. the peak at $\sim 0.75 \mathrm{ppm}$ from the cholate. 


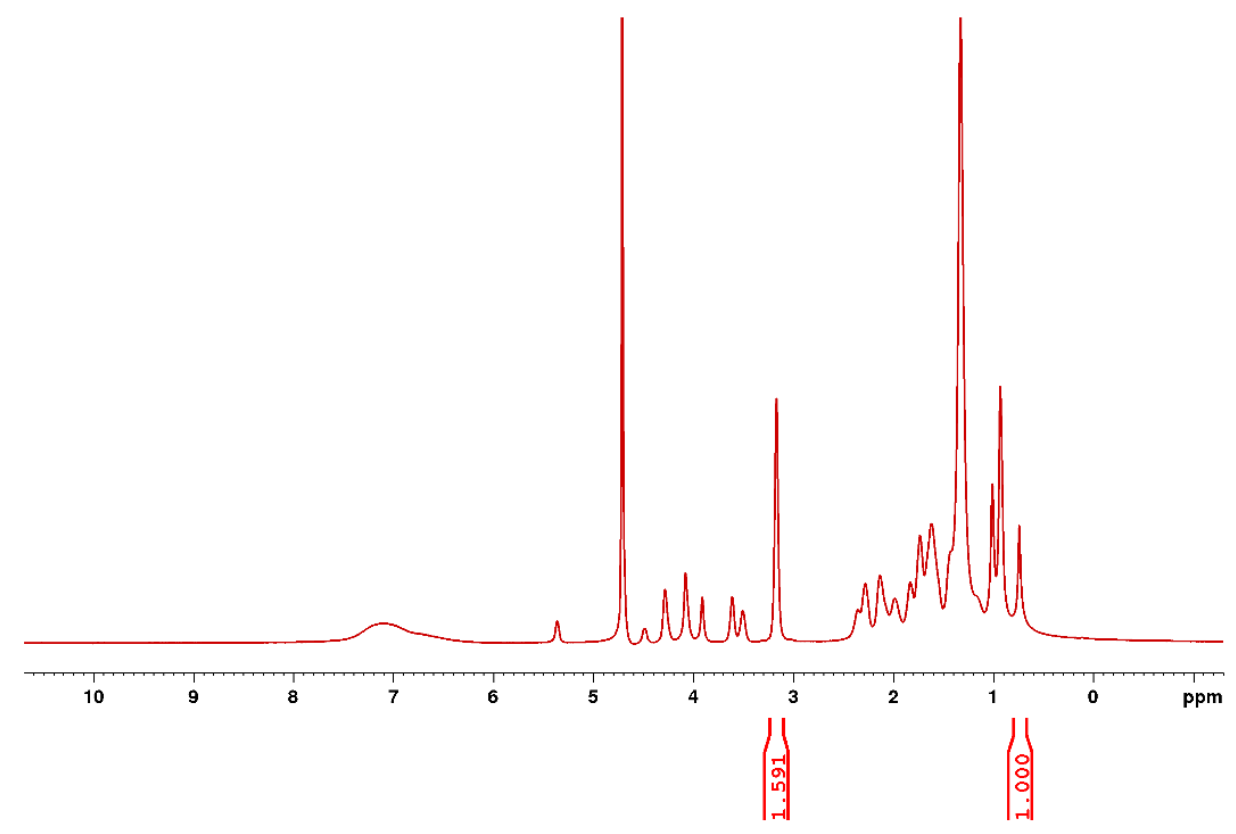

Figure 4.7. ${ }^{1}$ H-NMR spectra of SL25010 P:DMPC 1:1 (w/w) nanodiscs. Samples are recorded in the presence of 10 $\mathrm{mM}$ sodium cholate. To assess the molar concentration, the peak associated with quaternary ammonium protons from DMPC $(\sim 3.3 \mathrm{ppm})$ is integrated vs. the peak at $\sim 0.75 \mathrm{ppm}$ from the cholate.

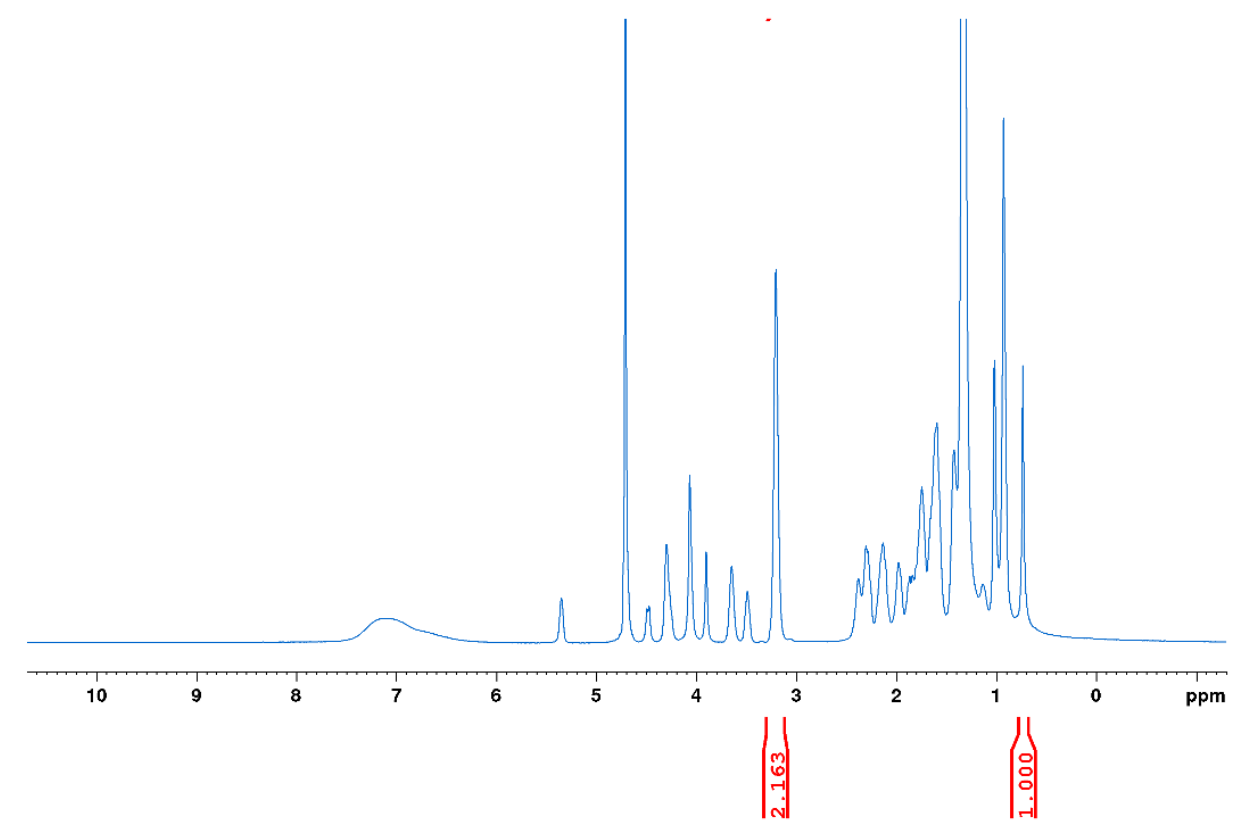

Figure 4.8. ${ }^{1}$ H-NMR spectra of SL30010 P:DMPC 1:1 (w/w) nanodiscs. Samples are recorded in the presence of 10 $\mathrm{mM}$ sodium cholate. To assess the molar concentration, the peak associated with quaternary ammonium protons from DMPC $(\sim 3.3 \mathrm{ppm})$ is integrated vs. the peak at $\sim 0.75 \mathrm{ppm}$ from the cholate. 


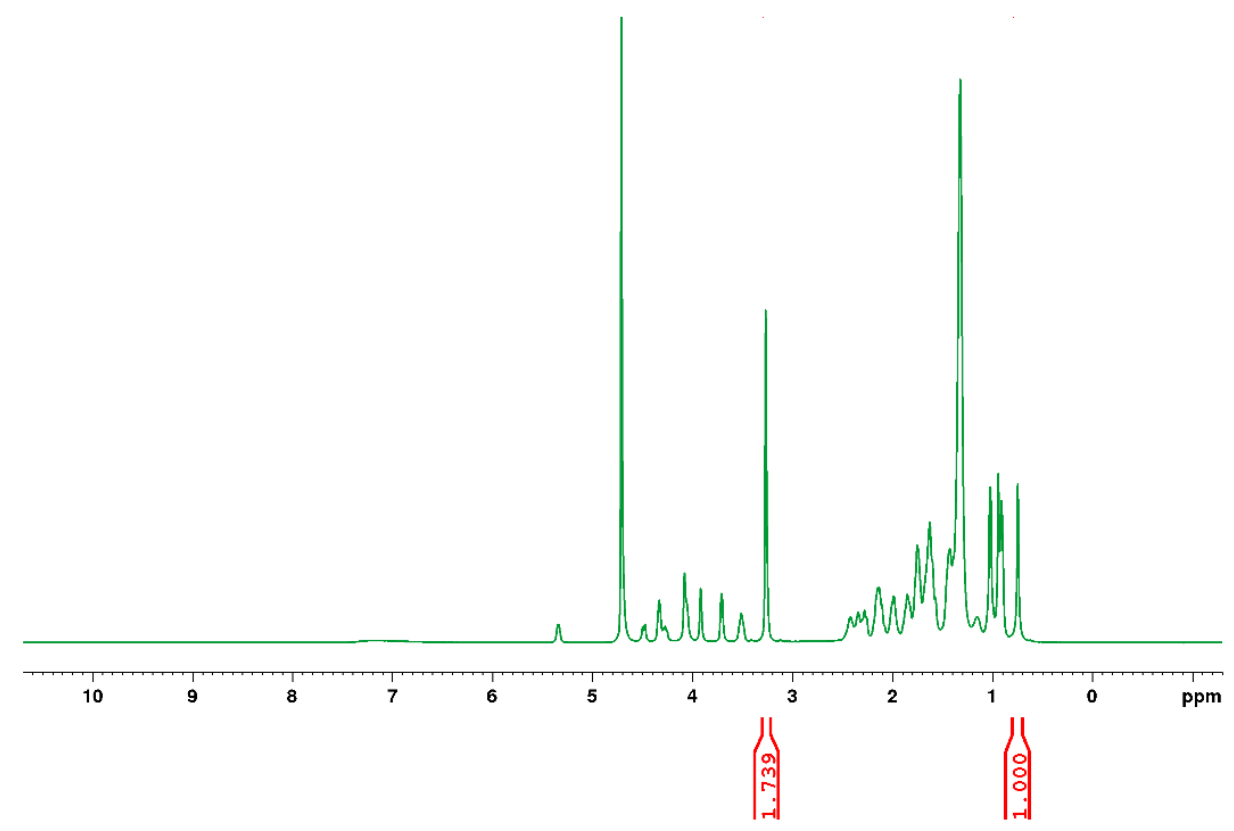

Figure 4.9. ${ }^{1}$ H-NMR spectra of SL40005 P:DMPC 1:1 (w/w) nanodiscs. Samples are recorded in the presence of 10 $\mathrm{mM}$ sodium cholate. To assess the molar concentration, the peak associated with quaternary ammonium protons from DMPC ( 3.3 ppm) is integrated vs. the peak at $\sim 0.75 \mathrm{ppm}$ from the cholate.

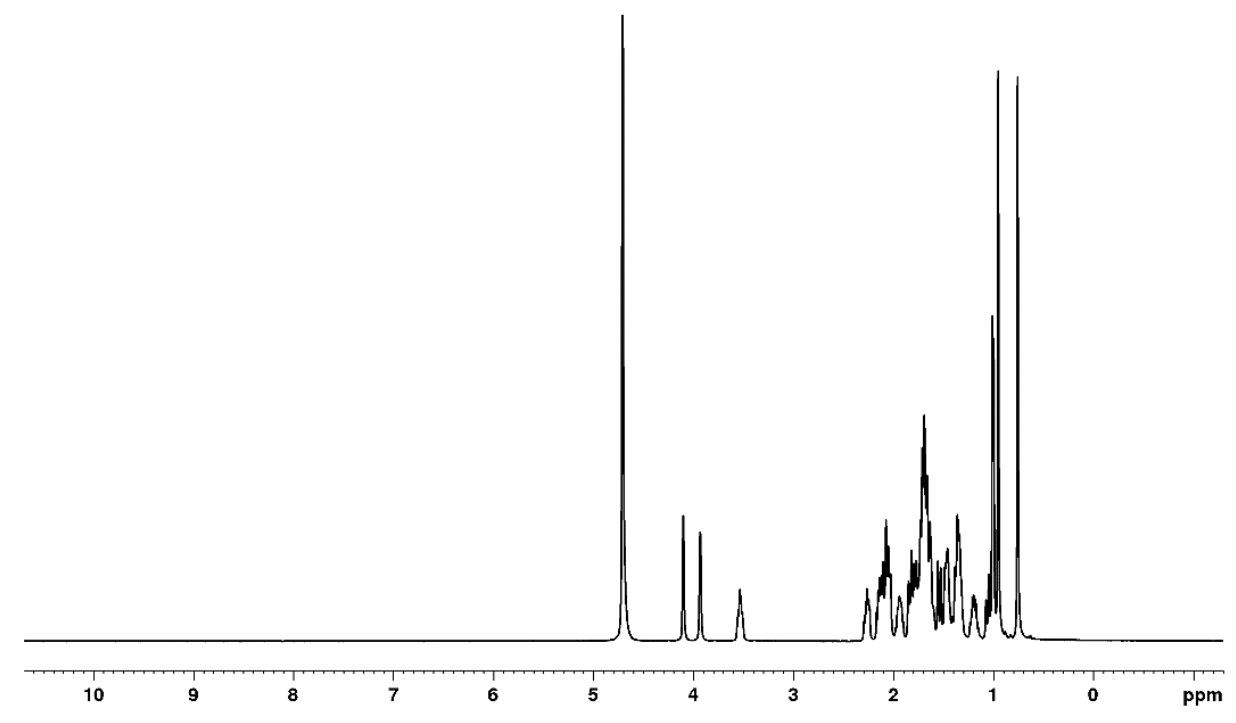

Figure 4.10. ${ }^{1} \mathrm{H}-\mathrm{NMR}$ spectra of $10 \mathrm{mM}$ Sodium Cholate in $\mathbf{1 0 0} \% \mathrm{D} 2 \mathrm{O}$. The peak used for integration is at $0.75 \mathrm{ppm}$, corresponding to the methyl group $\mathrm{C} 18$. 
f) Atomic Force Microscopy (AFM) coupled with Micro-Raman. AFM measurements were done in alternate contact mode with a SiC tip of $2.8 \mathrm{~N} / \mathrm{m}$, using a Witec Alpha $300 \mathrm{RA}$. Images were acquired in 256 points by 256 lines, with maps $5 \times 5 \mu^{2}$. Micro-Raman spectra were acquired using Witec Alpha 300 RA that employs a $532 \mathrm{~nm}$ laser operating at $20 \mathrm{~mW}$. Two nanodiscs solutions, $1 \mathrm{mg} / \mathrm{mL}$ and $0.001 \mathrm{mg} / \mathrm{mL}$ were dropped cast on a flat bare Silicon substrate, cleaned with a $4 \% \mathrm{v} / \mathrm{v}$ HF solution, and then dried at $30{ }^{\circ} \mathrm{C}$. Raman experiments were performed on the same samples. The Raman spectrum is shown in Figures 4.11, while AFM figures and depth profiles are reported in Figure 4.15.

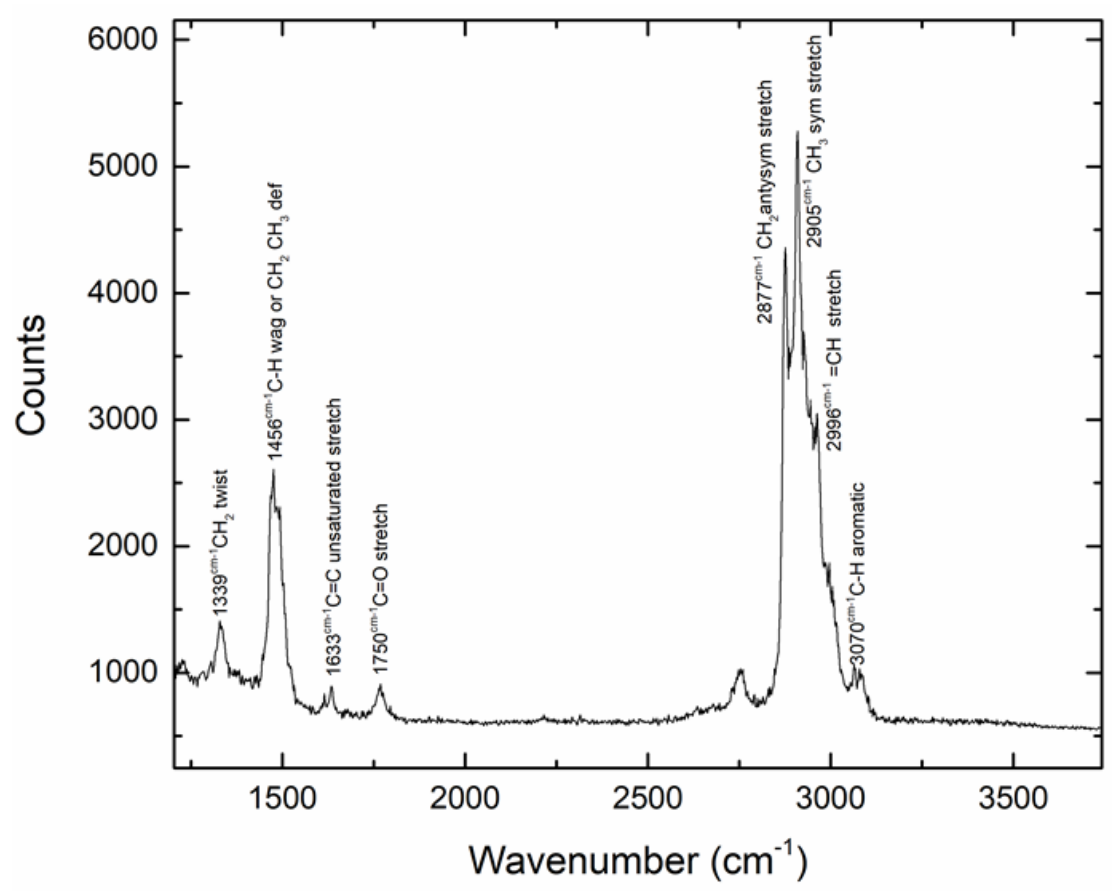

Figure 4.11. Raman vibrational spectrum of the $1 \mathrm{mg} / \mathrm{mL}$ unpurified SMA-EA:DMPC 1:1 (w/w) nanodiscs solution sample shown in Figure 4.15. 


\subsection{Results and Discussion}

In this study, we use a set of amphipathic copolymers to systematically investigate how the copolymer's solution physicochemical properties can be associated with the features of copolymerlipid nanodiscs, to increase nanodiscs' performances and to assist the design of new copolymers. It is accepted that copolymer-lipid nanodiscs solutions are stable under a wide range of conditions ${ }^{27,42,47}$. However, evidence suggests that despite the temporal stability, these nanoparticles show a high degree of dynamism as simplified in the scheme presented in Figure 4.12b.

\section{a) Copolymer structures}

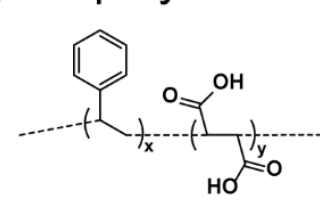

SL25010 P

SL30010 P

SL40005 P

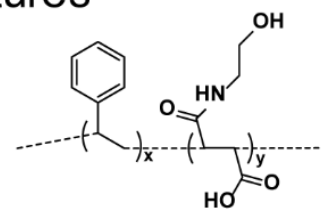

SMA-EA

\section{b) Copolymer-lipids nanodiscs}

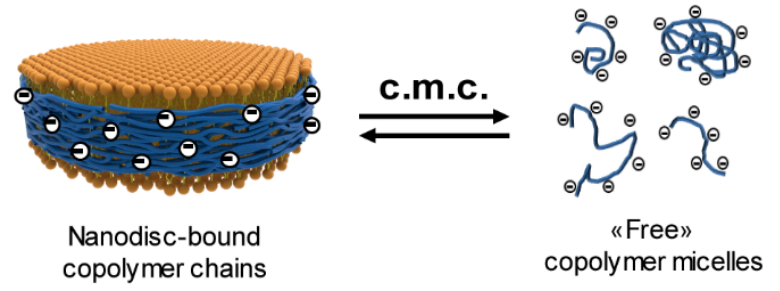

Figure 4.12. Chemical structures of SMA-based copolymers and copolymer-lipid nanodiscs. a) Chemical structured of the copolymers used in this study. The chemical structure of XIRAN ${ }^{\circledR}$ copolymers (SL25010 P, SL30010 P, SL40005 P) by Polyscience ${ }^{\circledR}$ are shown on the left and differ from each other for molecular weight and styrene-to-maleic acid (x:y) molar ratio. SMA-EA is a functionalized, low-molecular-weight derivative of SMA with a reduced charge density ${ }^{36}$. More details are provided in Table 1. All structures are shown in their fully protonated form. b) Simplified schematic representation of the chemical equilibrium between «nanodisc-bound» and «unbound/free-micellar» copolymer chains.

It has been demonstrated that copolymer nanodiscs can exchange phospholipids ${ }^{48-50}$, but the exchange of copolymer chains cannot be neglected ${ }^{51}$. This phenomenon elapses either among nanodiscs due to collisions or among the polymer chains bound in the nanodiscs and those free in solution as micelles. To the latter, copolymer chains can be coiled or elongated due to changes in the parameters such as $\mathrm{pH}$, ionic strength, temperature, and pressure ${ }^{43,52}$. Copolymer micelles can be intra-chain, interchains, or mixed. Additionally, the inevitable dispersity of any polymer sample can complicate the

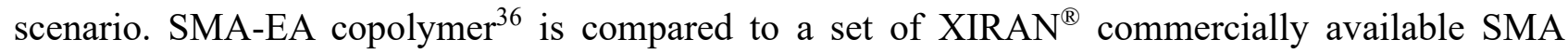


copolymer formulations $^{53}$. Figure $4.12 \mathrm{a}$ shows the structural similarities among the copolymers, while Table 4.1 provides each formulation's technical properties. Due to the amphipathic nature, SMA copolymers and derivatives form self-assembled macromolecular aggregates. The c.m.c. is a crucial value to assess amphipathic molecules. Pyrene, a four-ring polycyclic aromatic hydrocarbon (PAH), is known to be a convenient fluorescent probe for determining the c.m.c. of amphipathic aggregates ${ }^{46,54}$. Pyrene is strongly hydrophobic and shows a minimal solubility in water $(\sim 2-3 \mu \mathrm{M})^{54}$. Therefore, it easily inserts in the hydrophobic core of amphipathic micelle-like aggregates and the copolymer-lipid nanodiscs. Upon dilution, the micellar aggregate is destroyed, and pyrene molecules are directly exposed to water. Because of the solvent-dependent vibronic fine structure intensities shown by monomeric pyrene ${ }^{46,54}$, this event is profitably employed in fluorescence probe studies of amphipathic aggregates.

\begin{tabular}{ccccc}
\hline Copolymer & MW (kDa) & $\begin{array}{c}\mathbf{x : y} \\
\text { (molar ratio) }\end{array}$ & $\begin{array}{c}\mathbf{x}_{\mathbf{0}} \\
\text { (mg/mL) }\end{array}$ & $\mathbf{R}^{\mathbf{2}}$ \\
\hline SMA-EA & $\sim 2$ & $\sim 1.3: 1$ & 0.23 & 0.992 \\
SL25010 P & $\sim 10$ & $\sim 3: 1$ & 0.038 & 0.999 \\
SL30010 P & $\sim 7.5$ & $\sim 2.3: 1$ & 0.26 & 0.998 \\
SL40005 P & $\sim 5$ & $\sim 1.4: 1$ & 27 & 0.999 \\
\hline
\end{tabular}

Table 4.1. Physicochemical data of the investigated SMA copolymers. Spanning a range of molecular weights that goes from $\sim 2$ to $\sim 10 \mathrm{kDa}$, each copolymer has comparable dispersity $\left(\Theta=\mathrm{M}_{\mathrm{w}} / \mathrm{M}_{\mathrm{n}}>2.5\right.$ ) but different hydrophobicity/hydrophilicity ratios, as suggested from the styrene-to-maleic acid ratio. Additionally, SMA-EA shows a modified charge-density because of its modification if compared to any SMA-equivalent. The experimental curves $\mathrm{I}_{1} / \mathrm{I}_{3}$ vs. Log C shown in Figure 4.13a were fitted to a sigmoidal function. The c.m.c. values for each copolymer are obtained by taking the flex $\left(\mathrm{x}_{0}\right)$ of each fitted curve and converted from a decimal logarithmic scale to a linear scale. The coefficient of determination, $\mathrm{R}^{2}$, is also shown for each fitting. Experimental conditions are available in the «Materials and Methods» section.

Figure 4.13 shows a selection of spectra that highlights the spectral changes of pyrene containing solutions. When pyrene molecules are in the hydrophobic environment offered by copolymeric micelle-like aggregates (Figure 4.13a) or in the copolymer-lipid nanodiscs (Figure 4.13b), pyrene fluorescent spectra are of low intensity. Similarities were observed among the investigated copolymers and the copolymer-lipid nanodiscs. The «shoulder» after $400 \mathrm{~nm}$ for SL40005 P copolymer (green spectrum in Figure 4.13a) is due to scattering attributed to the high concentration used. The fluorescent spectrum of pyrene in the buffer solution (purple spectrum) simulates the infinite-dilution plateau or complete absence of amphipathic species. The crowding in hydrophobic environments justifies the quenching of pyrene fluorescence. By plotting the ratio between the 
intensities of the first and third fluorescence peaks, respectively labeled as $I_{1}$ and $I_{3}$, against the decimal logarithm of the concentration, it is possible to obtain the c.m.c. values.

\section{a) «Free» copolymers}

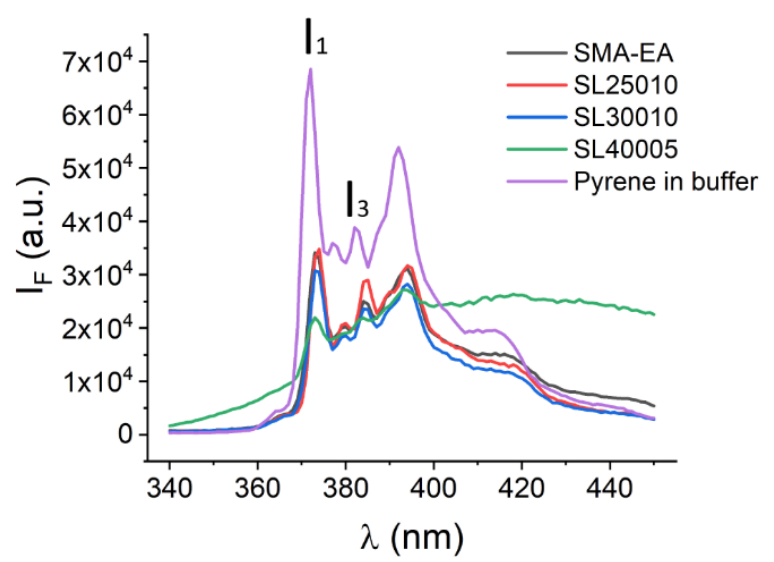

b) Unpurified copolymer-nanodiscs

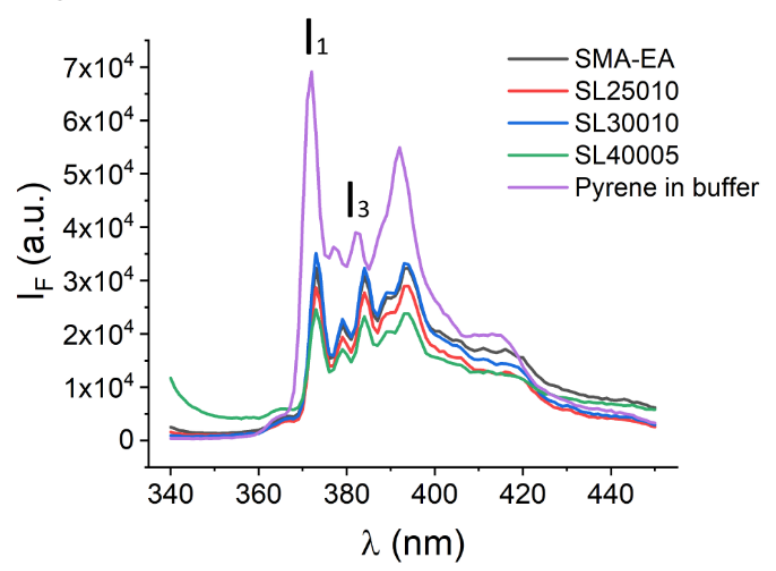

Figure 4.13. Pyrene fluorescence spectra for (a) «free» copolymers and (b) copolymer-lipid nanodises upon excitation at $331.5 \mathrm{~nm}$. a) Fluorescence spectra of pyrene in the presence of SMA-EA (black line), SL25010 P (red line), SL30010 P (blue line), or SL40005 (green line) copolymers «free» in solution and at the highest concentration used in this study. b) Fluorescence spectra of pyrene in the presence of unpurified SMA-EA-based nanodiscs (black line), SL25010 P based nanodiscs (red line), SL30010 P based nanodiscs (blue line), or SL40005 P (green line) at the highest concentration used in this study. In purple, both a) and b) shows the fluorescence spectrum of $1 \mu \mathrm{M}$ pyrene and can be associated with the most diluted sample, or the «infinite dilution sample.» Each sample was prepared in $10 \mathrm{mM}$ ammonium acetate buffer at $\mathrm{pH} 7$ at $25^{\circ} \mathrm{C}$, recorded at $25^{\circ} \mathrm{C}$, and pyrene concentration was kept constant at $1 \mu \mathrm{M}$.

Figure 4.14 shows the plot of the ratio $\mathrm{I}_{1} / \mathrm{I}_{3}$ for solutions of copolymers against the decimal logarithmic concentration of copolymers (Figure 4.14a) and unpurified copolymer-lipid nanodiscs (Figure 4.14b).

Results shown in Figure 4.14a are supported by the previous knowledge on the structure-property relationships ${ }^{55}$ and agree with previously reported results ${ }^{35}$, and the physicochemical properties are 
summarized in Table 4.1. On the one hand, SL40005 P is the most hydrophilic formulation due to the relatively low molecular weight $(\mathrm{MW} \sim 5 \mathrm{kDa})$, the low content of styrene repeating units $(\sim 1.4: 1)$, and the associated high charge density. SL25010 P is the heaviest copolymer (MW 10 kDa) and the most hydrophobic because of the 3:1 styrene-to-maleic acid molar ratio, resulting in a low charge density. For the same reasons, SL30010 P is placed in an intermediate position among these two cases (MW 7.5 kDa; 2.3:1).

\section{a) «Free» copolymers}

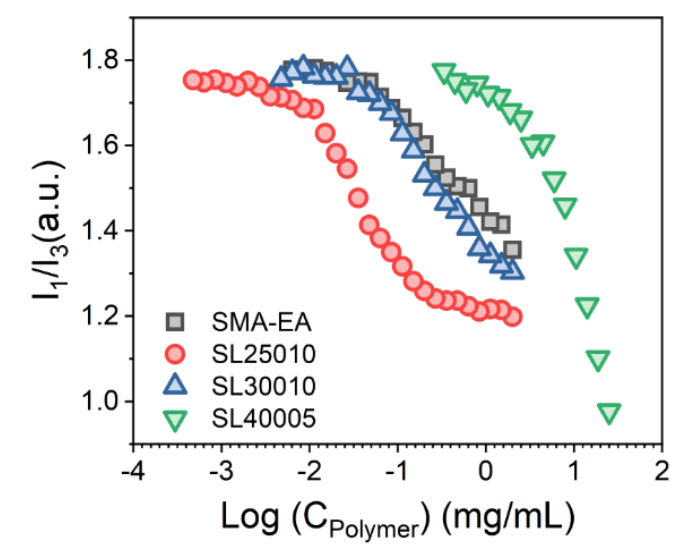

\section{b) Unpurified copolymer-nanodiscs}

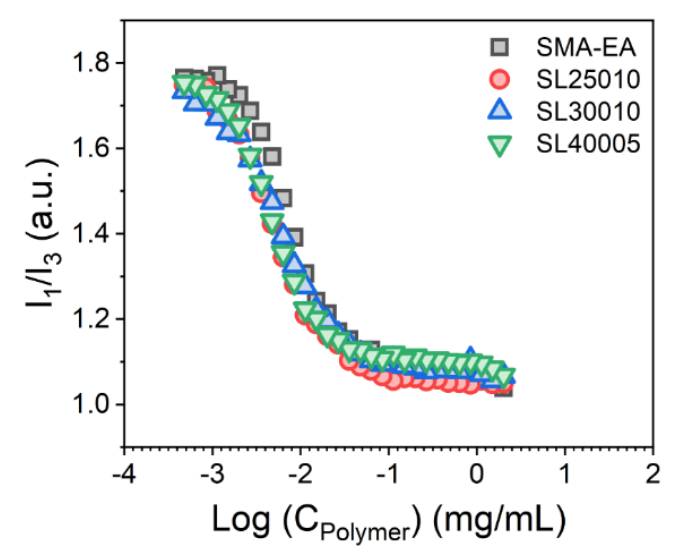

Figure 4.14. Copolymers show drastic changes in the presence of phospholipids. a) Plots of the pyrene $I_{1} / I_{3}$ ratio against the decimal logarithm of the copolymers' concentration in $\mathrm{mg} / \mathrm{mL}$. As expected from the physicochemical properties listed in Table 4.1, the polymer SL25010 P (circles in red) results in the most hydrophobic, while SL40005 P (triangles in green) is the most hydrophilic. SMA-EA (black squares) and SL30010 P (blue triangles) show comparable responses. b) Plots of the pyrene $I_{1} / I_{3}$ ratio against the concentration of copolymer-lipid nanodiscs in solution. The concentration is reported as a function of the polymer concentration's decimal logarithm in $\mathrm{mg} / \mathrm{mL}$. Interestingly, the differences observed in a) become negligible among all the copolymers when interacting with lipids. Both a) and b) show the average of three experiments obtained from independent samples. Each sample was prepared in $10 \mathrm{mM}$ ammonium acetate buffer at $\mathrm{pH} 7$ at $25^{\circ} \mathrm{C}$, recorded at $25^{\circ} \mathrm{C}$, and pyrene concentration was kept constant at $1 \mu \mathrm{M}$. Data, error bars, and fitting are reported in Figures 4.1 and 4.2. 
Given the similarities among all the formulations, AFM experiments were performed on SMAEA:DMPC 1:1 (w/w) unpurified nanodiscs. Previous studies confirm that the adhesion among phosphocholine polar headgroups is responsible for the formation of stacked structures ${ }^{56}$. AFM images shown in Figures 4.15a-b were respectively obtained by drop cast of copolymer-lipid nanodiscs solutions at a concentration above and below the copolymer's c.m.c.

a)

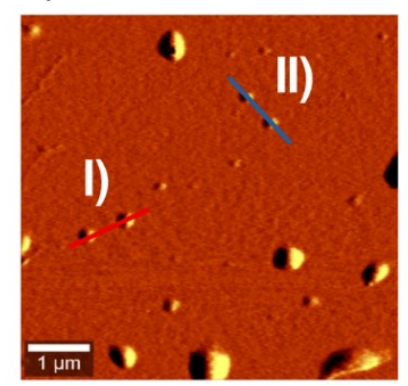

b)
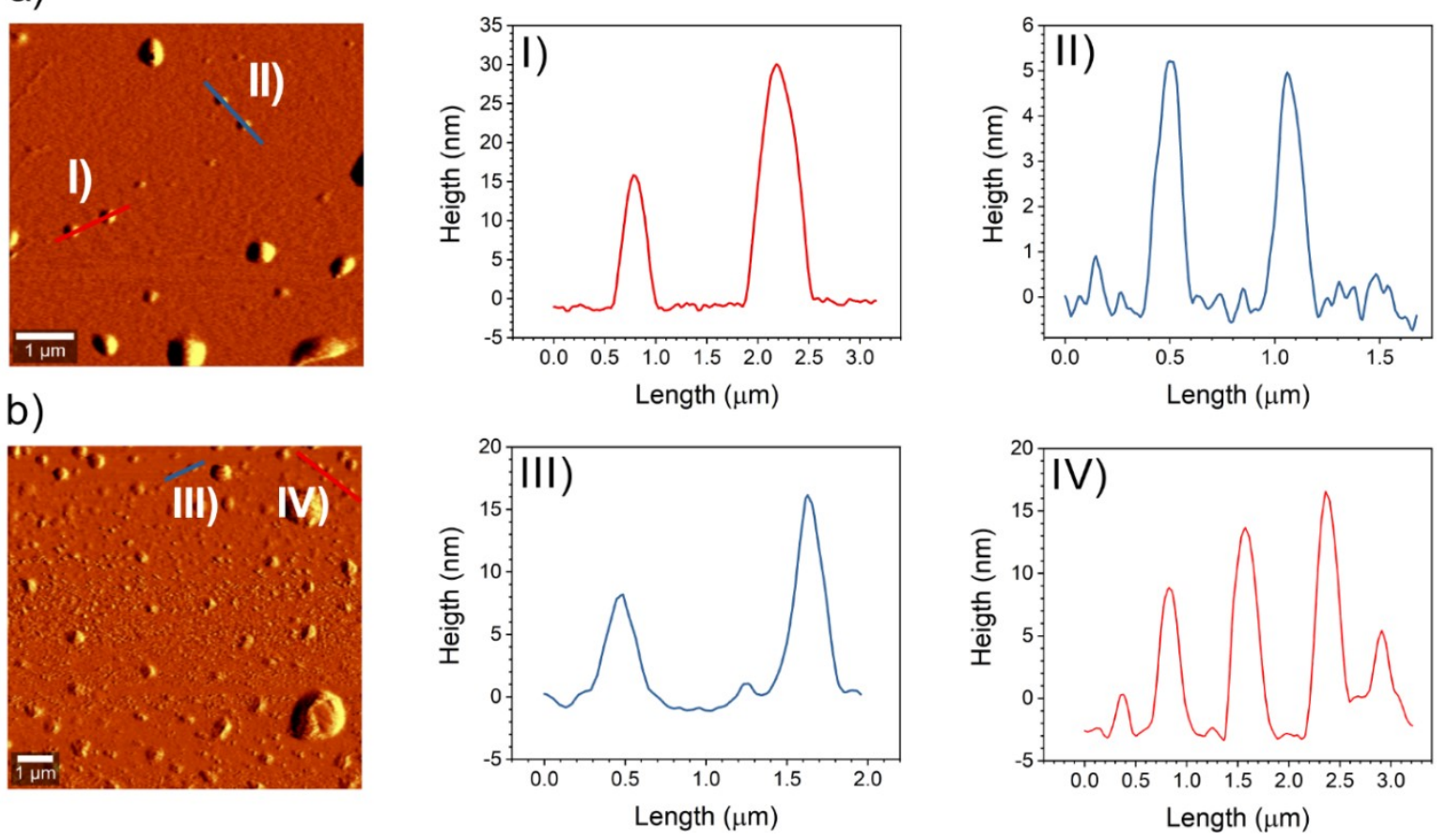

Figure 4.15. AFM images and depth profiles above and below the c.m.c. of SMA-EA copolymer. a) AFM image of a sample of $1 \mathrm{mg} / \mathrm{mL}$ DMPC:SMA-EA 1:1 w/w. I) and II) are the depth profiles indicated in a). b) AFM image of a sample of $0.001 \mathrm{mg} / \mathrm{mL}$ DMPC:SMA-EA 1:1 w/w. III) and IV) are the depth profiles indicated in b). The Raman spectrum is shown in Figure 4.11.

The depth of DMPC bilayers is $\sim 5 \mathrm{~nm}$. Depth profiles I) and II) are shown in Figure 4.15a and were obtained at $1 \mathrm{mg} / \mathrm{mL}$. At such a high nanodiscs concentration, stacks of DMPC nanodiscs were observed. For a 1000-times more diluted concentration, i.e., below SMA-EA's copolymer c.m.c., the depth profiles III) and IV) shown in Figure 4.15b confirms the existence of nanoparticles compatible with the height of DMPC bilayers and diameter compared to the size of SMA-EA-DMPC 1:1 w/w nanodiscs reported in the literature ${ }^{36}$. Raman vibrational experiments were used to confirm the presence of both copolymer and lipids in the $1 \mathrm{mg} / \mathrm{mL}$ sample used for AFM measurements (Figure 4.11). 


\begin{tabular}{|c|c|c|c|c|c|c|c|c|c|}
\hline \multirow[b]{2}{*}{ Copolymer } & \multicolumn{3}{|c|}{ Unpurified nanodiscs } & \multicolumn{3}{|c|}{ Unpurified nanodiscs } & \multicolumn{3}{|c|}{ Purified nanodiscs } \\
\hline & $\begin{array}{c}\mathbf{x}_{0} \\
(\log C)\end{array}$ & $\begin{array}{c}\mathbf{x}_{0} \\
(\mathrm{mg} / \mathrm{mL})\end{array}$ & $\mathbf{R}^{2}$ & $\begin{array}{c}\mathbf{x}_{0} \\
(\log [\mathrm{DMPC}])\end{array}$ & $\begin{array}{c}\mathbf{x} 0 \\
(\mathbf{m M})\end{array}$ & $\mathbf{R}^{2}$ & $\begin{array}{c}\mathrm{x}_{0} \\
(\log [\mathrm{DMPC}])\end{array}$ & $\begin{array}{c}\mathbf{x} 0 \\
(\mathbf{m M})\end{array}$ & $\mathbf{R}^{2}$ \\
\hline SMA-EA & -2.12 & $7.59 \times 10^{-3}$ & 0.994 & -1.95 & $1.12 \times 10^{-2}$ & 0.994 & -1.60 & $2.51 \times 10^{-2}$ & 0.999 \\
\hline SL25010 P & -2.27 & $5.37 \times 10^{-3}$ & 0.998 & -2.10 & $7.94 \times 10^{-3}$ & 0.998 & -1.53 & $2.95 \times 10^{-2}$ & 0.998 \\
\hline SL30010 P & -2.50 & $3.16 \times 10^{-3}$ & 0.979 & -2.33 & $4.68 \times 10^{-3}$ & 0.979 & -1.55 & $2.82 \times 10^{-2}$ & 0.998 \\
\hline SL40005 P & -2.31 & $4.90 \times 10^{-3}$ & 0.999 & -2.14 & $7.24 \times 10^{-3}$ & 0.999 & -1.68 & $2.09 \times 10^{-2}$ & 0.999 \\
\hline
\end{tabular}

Table 4.2. Copolymer-lipid nanodiscs data: unpurified vs. purified. From left-to-right, the chart shows the data plotted, respectively, in Figures 4.2-4.4. The polymer-based nanodiscs were obtained by mixing the lipids and the copolymers in a 1:1 weight ratio, as detailed in the «Materials and Methods» section. Unpurified nanodiscs appear twice; as a function of the polymer concentration $(\mathrm{C}$ in $\mathrm{mg} / \mathrm{mL})$ and as a function of the DMPC concentration (mM). The «c.m.c.like» values for each copolymer-nanodiscs were obtained by taking the flex $\left(\mathrm{x}_{0}\right)$ of each fitted curve and converted from a decimal logarithmic scale to a linear range. The coefficient of determination, $\mathrm{R}^{2}$, is also shown for each fitting.

Among the resulting materials, copolymer chains that are in equilibrium with the nanodiscs-bound chains are discarded too. We speculate that this process, in addition to dilution, may alter the integrity of the nanodiscs per the principle of chemical equilibrium. Size exclusion chromatography experiments were used herein to purify samples of copolymer-lipid nanodiscs. In fact, when copolymers were added to phospholipids, together with the formation of nanodiscs, a plethora of other micellar aggregates were formed. Chromatograms of model copolymer-lipid nanodiscs, reported in Figure 4.5, show two main peaks. The first is narrow and associated with the nanodiscs. The second is broad and usually related to the excess of micellar polymer chains. Copolymer micelles can display a plethora of morphologies ${ }^{60}$, and mixed unstructured copolymer chains-lipids aggregates cannot be excluded. This provides a variety of potential dynamic subunits for higher-level assemblies that could explain the breadth of the second chromatographic peak. Despite the physicochemical differences in the copolymers, after the purification step, copolymer-lipid nanodiscs behave similarly to the unpurified counterpart, Figure $4.14 \mathrm{~b}$. Figure 4.16 shows a comparison between samples of unpurified and purified lipid nanodiscs for each used copolymer formulation. A more in-depth analysis is presented in Table 4.2.

It is observable how the flex $\left(\mathrm{x}_{0}\right)$, associated with the nanodiscs' c.m.c., shifts toward higher concentration values. After purification, with the removal of the micellar aggregates in solution, SMA-EA nanodiscs shifts by 2.2 times, while SL25010, SL30010, and SL40005 nanodiscs shift by 3.7, 6, and 2.9 times respectively. This supports the existence of chemical equilibrium as schematically represented in Figure 12b. Data with error bars and fitting are presented in Figures 4.3 and 4.4 . 

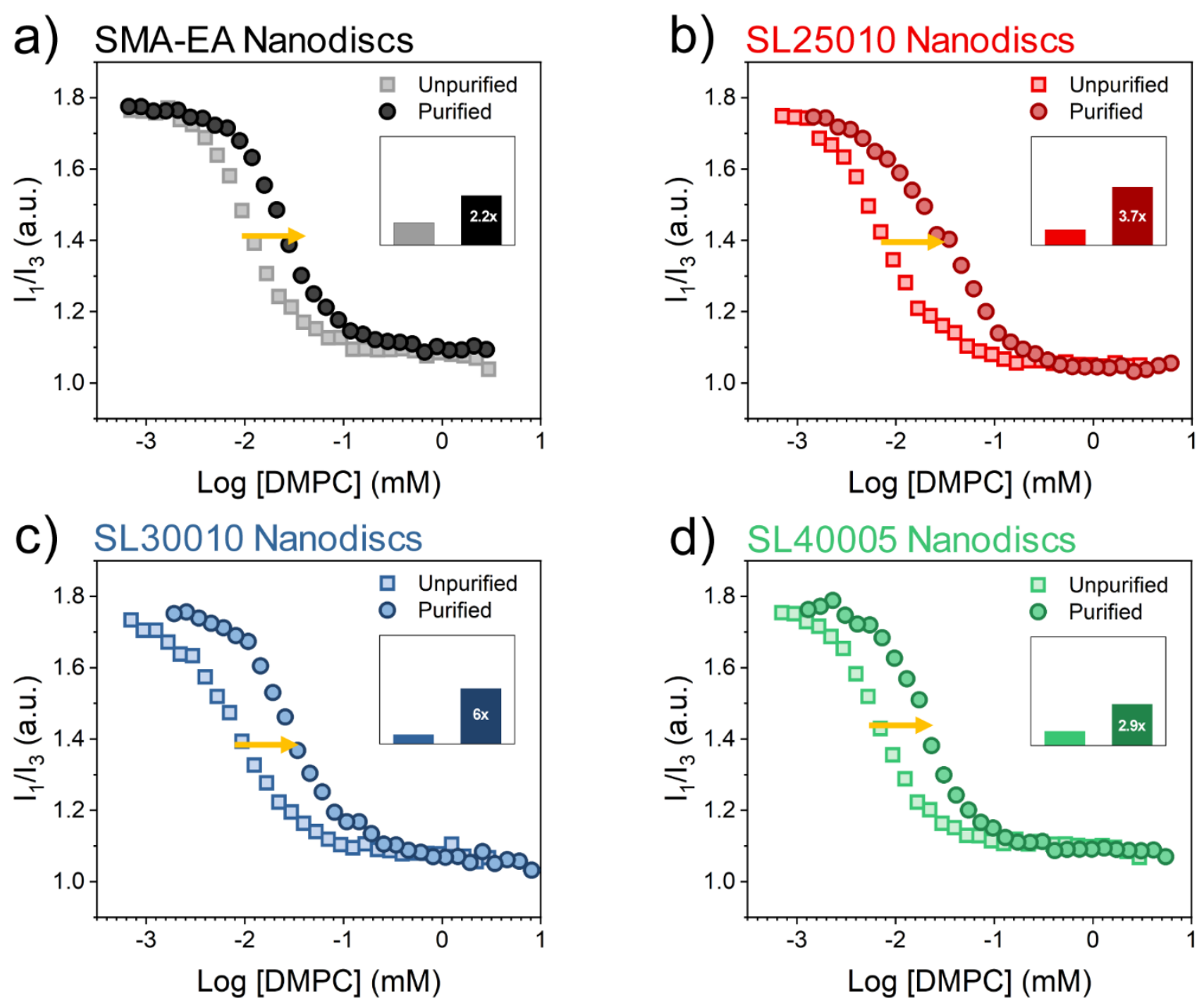

Figure 4.16 - Comparison of the pyrene $I_{1} / I_{3}$ ratio against the concentration of unpurified (squares) and purified (circles) copolymer-nanodises in solution. By purification through SEC, removing the micellar polymer not bound to the nanodiscs edge causes a consistent horizontal shift for all of the copolymer formulations investigated, as reported from a) to d). The micellar polymer removed by SEC is either an excess or in chemical equilibrium among the states «nanodiscs-bound» and «unbound/free-micellar» in solution. Each sample was prepared in $10 \mathrm{mM}$ ammonium acetate buffer at $\mathrm{pH} 7$ at $25{ }^{\circ} \mathrm{C}$, recorded at $25^{\circ} \mathrm{C}$, and pyrene concentration was kept constant at $1 \mu \mathrm{M}$. Data, error bars, and fitting are reported in Figures 4.3 and 4.4. The concentration of SEC-purified copolymer-lipid nanodiscs was assessed through ${ }^{1} \mathrm{H}$ NMR using sodium cholate as an internal standard and reported in Figures 4.6-4.9. 


\subsection{Conclusions}

In this study, we report the use of an analytic assay to assess the c.m.c. of SMA-based copolymers and to find connections among the c.m.c. and the integral stability of the nanodiscs intended as nanoparticles. The major findings of this study are: (i) The c.m.c. of SMA-EA copolymer, produced in our laboratory, is reported for the first time compared to some commercially available SMA copolymer formulations (XIRAN®). (ii) The interaction with phospholipids drastically alters the copolymer's c.m.c. values. When in the form of nanodiscs, it is impossible to discern among pure copolymer's c.m.c. and pure phospholipid contributions, making it necessary to use a more generic concept such as the nanodiscs c.m.c. (iii) Pieces of evidence suggest the existence of a chemical equilibrium among the «free» or «micellar» copolymer chains and the «nanodiscs-bound» copolymer chains. Further investigations to evaluate the effect of the removal of free/micellar copolymer on the stability of nanodiscs (unpurified vs. purified behavior) are required to characterize the properties of polymers and nanodiscs fully. We speculate that this phenomenon may be potentially exploited to exchange belts in samples of copolymer-lipid nanodiscs. Indeed, a given formulation can be more successful in the extraction but need not be suitable for some biophysical or biochemical techniques. Additionally, a more inexpensive copolymer can be used to extract successfully and stabilize membrane proteins and then be substituted with tagged copolymers ${ }^{19,40,61,62}$, a more expensive solution, optimizing costs, and benefits.

\section{Acknowledgments}

This research was supported by the National Institute of Health (GM084018 to AR). Research in the CLR laboratory is supported by the University of Catania Departmental Project 2017-2020 (Progetto di Dipartimento 2017-2020). AFM and Raman measurements were performed by MC at the BioNanotech Research and Innovation Tower (BRIT), University of Catania, Italy.

A special thanks go to Professor Carmelo La Rosa and Dr. Marcello Condorelli at the University of Catania, Sicily, Italy, for the valuable discussions in discussing this study and for the help with AFM measurements.

We also grateful to Polyscience ${ }^{\circledR}$ (Geleen, Netherlands) for gifting the formulations of XIRAN ${ }^{\circledR}$ SL25010 P, SL30010 P, and SL40005 P. Finally, we thank Dr. Thirupathi Ravula for synthesis and characterization of SMA-EA copolymer used in this study, and the Ramamoorthy group for the many discussions in this study and data interpretation. 


\section{References}

(1) Overington, J. P.; Al-Lazikani, B.; Hopkins, A. L. How Many Drug Targets Are There? Nat. Rev. Drug Discov. 2006, 5 (12), 993-996.

(2) Yeagle, P. L. Non-Covalent Binding of Membrane Lipids to Membrane Proteins. Biochim. Biophys. Acta - Biomembr. 2014, 1838 (6), 1548-1559.

(3) Sun, C.; Benlekbir, S.; Venkatakrishnan, P.; Wang, Y.; Hong, S.; Hosler, J.; Tajkhorshid, E.; Rubinstein, J. L.; Gennis, R. B. Structure of the Alternative Complex III in a Supercomplex with Cytochrome Oxidase. Nature 2018, 557 (7703), 123-126.

(4) Hagn, F.; Etzkorn, M.; Raschle, T.; Wagner, G. Optimized Phospholipid Bilayer Nanodiscs Facilitate High-Resolution Structure Determination of Membrane Proteins. J. Am. Chem. Soc. 2013, 135 (5), 1919-1925.

(5) Simon, K. S.; Pollock, N. L.; Lee, S. C. Membrane Protein Nanoparticles: The Shape of Things to Come. Biochem. Soc. Trans. 2018, 46 (6), 1495-1504.

(6) Parmar, M. J.; De, C.; Lousa, M.; Muench, S. P.; Goldman, A.; Postis, V. L. G. Artificial Membranes for Membrane Protein Purification, Functionality and Structure Studies. Biochem. Soc. Trans. 2016, 44, 877-882.

(7) Lee, S. C.; Knowles, T. J.; Postis, V. L. G.; Jamshad, M.; Parslow, R. A.; Lin, Y.; Goldman, A.; Sridhar, P.; Overduin, M.; Muench, S. P.; et al. A Method for Detergent-Free Isolation of Membrane Proteins in Their Local Lipid Environment. Nat. Protoc. 2016, 11 (7), 1149-1162.

(8) Knowles, T. J.; Finka, R.; Smith, C.; Lin, Y.-P.; Dafforn, T.; Overduin, M. Membrane Proteins Solubilized Intact in Lipid Containing Nanoparticles Bounded by Styrene Maleic Acid Copolymer. J. Am. Chem. Soc. 2009, 131 (22), 7484-7485.

(9) Rajesh, S.; Knowles, T.; Overduin, M. Production of Membrane Proteins without Cells or 
Detergents. N. Biotechnol. 2011, 28 (3), 250-254.

(10) Jamshad, M.; Lin, Y.-P.; Knowles, T. J.; Parslow, R. A.; Harris, C.; Wheatley, M.; Poyner, D. R.; Bill, R. M.; Thomas, O. R. T.; Overduin, M.; et al. Surfactant-Free Purification of Membrane Proteins with Intact Native Membrane Environment. Biochem. Soc. Trans. 2011, $39,813-818$.

(11) Hardin, N. Z.; Ravula, T.; Mauro, G. Di; Ramamoorthy, A. Hydrophobic Functionalization of Polyacrylic Acid as a Versatile Platform for the Development of Polymer Lipid Nanodisks. Small 2019, 15 (9).

(12) Yasuhara, K.; Arakida, J.; Ravula, T.; Ramadugu, S. K.; Sahoo, B.; Kikuchi, J.-I.; Ramamoorthy, A. Spontaneous Lipid Nanodisc Fomation by Amphiphilic Polymethacrylate Copolymers. J Am Chem Soc 2017, 139, 18657-18663.

(13) Ravula, T.; Hardin, N. Z.; Bai, J.; Im, S. C.; Waskell, L.; Ramamoorthy, A. Effect of Polymer Charge on Functional Reconstitution of Membrane Proteins in Polymer Nanodiscs. Chem. Commun. 2018, 54 (69), 9615-9618.

(14) Dörr, J. M.; Scheidelaar, S.; Koorengevel, M. C.; Dominguez, J. J.; Schäfer, M.; van Walree, C. A.; Killian, J. A. The Styrene-Maleic Acid Copolymer: A Versatile Tool in Membrane Research. Eur. Biophys. J. 2016, 45 (1), 3-21.

(15) Ravula, T.; Hardin, N. Z.; Di Mauro, G. M.; Ramamoorthy, A. Styrene Maleic Acid Derivates to Enhance the Applications of Bio-Inspired Polymer Based Lipid-Nanodiscs. Eur. Polym. J. 2018, 108, 597-602.

(16) Overduin, M.; Klumperman, B. Advancing Membrane Biology with Poly(Styrene-Co-Maleic Acid)-Based Native Nanodiscs. Eur. Polym. J. 2019, 110, 63-68.

(17) Hall, S. C. L.; Tognoloni, C.; Price, G. J.; Klumperman, B.; Edler, K. J.; Dafforn, T. R.; Arnold, T. Influence of Poly(Styrene- Co -Maleic Acid) Copolymer Structure on the Properties and Self-Assembly of SMALP Nanodiscs. Biomacromolecules 2018, 19 (3), 761-772.

(18) Oluwole, A. O.; Danielczak, B.; Meister, A.; Babalola, J. O.; Vargas, C.; Keller, S. Solubilization of Membrane Proteins into Functional Lipid-Bilayer Nanodiscs Using a Diisobutylene/Maleic Acid Copolymer. Angew. Chemie - Int. Ed. 2017, 56 (7), 1919-1924.

(19) Lindhoud, S.; Carvalho, V.; Pronk, J. W.; Aubin-Tam, M. E. SMA-SH: Modified StyreneMaleic Acid Copolymer for Functionalization of Lipid Nanodiscs. Biomacromolecules 2016, 
$17(4), 1516-1522$.

(20) Brady, N. G.; Qian, S.; Bruce, B. D. Analysis of Styrene Maleic Acid Alternating Copolymer Supramolecular Assemblies in Solution by Small Angle X-Ray Scattering. Eur. Polym. J. 2019, 111 (June 2018), 178-184.

(21) Orwick-Rydmark, M.; Lovett, J. E.; Graziadei, A.; Lindholm, L.; Hicks, M. R.; Watts, A. Detergent-Free Incorporation of a Seven-Transmembrane Receptor Protein into Nanosized Bilayer Lipodisq Particles for Functional and Biophysical Studies. Nano Lett. 2012, 12 (9), 4687-4692.

(22) Morrison, K. A.; Akram, A.; Mathews, A.; Khan, Z. A.; Patel, J. H.; Zhou, C.; Hardy, D. J.; Moore-Kelly, C.; Patel, R.; Odiba, V.; et al. Membrane Protein Extraction and Purification Using Styrene-Maleic Acid (SMA) Copolymer: Effect of Variations in Polymer Structure. Biochem. J. 2016, 473 (23), 4349-4360.

(23) Gulamhussein, A. A.; Meah, D.; Soja, D. D.; Fenner, S.; Saidani, Z.; Akram, A.; Lallie, S.; Mathews, A.; Painter, C.; Liddar, M. K.; et al. Examining the Stability of Membrane Proteins within SMALPs. Eur. Polym. J. 2019, 112 (June 2018), 120-125.

(24) Krishnarjuna, B.; Ravula, T.; Ramamoorthy, A. Detergent-Free Extraction, Reconstitution and Characterization of Membrane-Anchored Cytochrome-B5 in Native Lipids. Chem. Commun. 2020, 56 (48), 6511-6514.

(25) Overduin, M.; Esmaili, M. Memtein: The Fundamental Unit of Membrane-Protein Structure and Function. Chem. Phys. Lipids 2019, 218, 73-84.

(26) Reading, E.; Hall, Z.; Martens, C.; Haghighi, T.; Findlay, H.; Ahdash, Z.; Politis, A.; Booth, P. J. Interrogating Membrane Protein Conformational Dynamics within Native Lipid Compositions. Angew. Chemie - Int. Ed. 2017, 56 (49), 15654-15657.

(27) Simon, K. S.; Pollock, N. L.; Lee, S. C. Membrane Protein Nanoparticles: The Shape of Things to Come. Biochemical Society Transactions. 2018, 1495-1504.

(28) Cuevas Arenas, R.; Klingler, J.; Vargas, C.; Keller, S. Influence of Lipid Bilayer Properties on Nanodisc Formation Mediated by Styrene/Maleic Acid Copolymers. Nanoscale 2016, 8 (32), $15016-15026$.

(29) Stroud, Z.; Hall, S. C. L.; Dafforn, T. R. Purification of Membrane Proteins Free from Conventional Detergents: SMA, New Polymers, New Opportunities and New Insights. 
Methods 2018, 147, 106-117.

(30) Huang, J.; Turner, S. R. Recent Advances in Alternating Copolymers: The Synthesis, Modification, and Applications of Precision Polymers. Polymer. May 2017, 572-586.

(31) Klumperman, B. Mechanistic Considerations on Styrene-Maleic Anhydride Copolymerization Reactions. Polymer Chemistry. 2010, 558-562.

(32) Lessard, B.; Marić, M. One-Step Poly(Styrene-Alt-Maleic Anhydride)-Block-Poly(Styrene) Copolymers with Highly Alternating Styrene/Maleic Anhydride Sequences Are Possible by Nitroxide-Mediated Polymerization. Macromolecules 2010, 43 (2), 879-885.

(33) Craig, A. F.; Clark, E. E.; Sahu, I. D.; Zhang, R.; Frantz, N. D.; Al-Abdul-Wahid, M. S.; Dabney-Smith, C.; Konkolewicz, D.; Lorigan, G. A. Tuning the Size of Styrene-Maleic Acid Copolymer-Lipid Nanoparticles (SMALPs) Using RAFT Polymerization for Biophysical Studies. Biochim. Biophys. Acta - Biomembr. 2016, 1858 (11), 2931-2939.

(34) Smith, A. A. A.; Autzen, H. E.; Laursen, T.; Wu, V.; Yen, M.; Hall, A.; Hansen, S. D.; Cheng, Y.; Xu, T. Controlling Styrene Maleic Acid Lipid Particles through RAFT. Biomacromolecules 2017, 18 (11), 3706-3713.

(35) Scheidelaar, S.; Koorengevel, M. C.; van Walree, C. A.; Dominguez, J. J.; Dörr, J. M.; Killian, J. A. Effect of Polymer Composition and PH on Membrane Solubilization by Styrene-Maleic Acid Copolymers. Biophys. J. 2016, 111 (9), 1974-1986.

(36) Ravula, T.; Ramadugu, S. K.; Di Mauro, G. M.; Ramamoorthy, A. Bioinspired, Size-Tunable Self-Assembly of Polymer-Lipid Bilayer Nanodiscs. Angew. Chemie Int. Ed. 2017, 56, $11466-11470$.

(37) Ravula, T.; Hardin, N. Z.; Ramadugu, S. K.; Ramamoorthy, A. PH Tunable and Divalent Metal Ion Tolerant Polymer Lipid Nanodiscs. Langmuir 2017, 33 (40), 10655-10662.

(38) Ravula, T.; Hardin, N. Z.; Ramadugu, S. K.; Cox, S. J.; Ramamoorthy, A. Formation of PHResistant Monodispersed Polymer-Lipid Nanodiscs. Angew. Chemie Int. Ed. 2018, 57 (5), $1342-1345$.

(39) Harding, B. D.; Dixit, G.; Burridge, K. M.; Sahu, I. D.; Dabney-Smith, C.; Edelmann, R. E.; Konkolewicz, D.; Lorigan, G. A. Characterizing the Structure of Styrene-Maleic Acid Copolymer-Lipid Nanoparticles (SMALPs) Using RAFT Polymerization for Membrane Protein Spectroscopic Studies. Chem. Phys. Lipids 2019, 218, 65-72. 
(40) Di Mauro, G. M.; Hardin, N. Z.; Ramamoorthy, A. Lipid-Nanodiscs Formed by Paramagnetic Metal Chelated Polymer for Fast NMR Data Acquisition. Biochim. Biophys. Acta-Biomembr. 2020, 1862 (9), 183332.

(41) Ravula, T.; Hardin, N. Z.; Ramamoorthy, A. Polymer Nanodiscs: Advantages and Limitations. Chem. Phys. Lipids 2019, 219, 45-49.

(42) Dörr, J. M.; Koorengevel, M. C.; Schäfer, M.; Prokofyev, A. V; Scheidelaar, S.; Van Der Cruijsen, E. A. W.; Dafforn, T. R.; Baldus, M.; Killian, J. A. Detergent-Free Isolation, Characterization, and Functional Reconstitution of a Tetrameric $\mathrm{K}^{+}$Channel: The Power of Native Nanodiscs. Proc. Natl. Acad. Sci. U. S. A. 2014, 111 (52), 18607-18612.

(43) Owen, S. C.; Chan, D. P. Y.; Shoichet, M. S. Polymeric Micelle Stability. Nano Today 2012, $7(1), 53-65$.

(44) Stetsenko, A.; Guskov, A. An Overview of the Top Ten Detergents Used for Membrane Protein Crystallization. Crystals 2017, 7 (7), 197.

(45) Mukerjee, P.; Mysels, K. J. Critical Micelle Concentrations of Aqueous Surfactant Systems; U.S. National Bureau of Standards; for sale by the Supt. of Docs., U.S. Govt. Print. Off.: Washington D.C., 1971.

(46) Basu Ray, G.; Chakraborty, I.; Moulik, S. P. Pyrene Absorption Can Be a Convenient Method for Probing Critical Micellar Concentration (Cmc) and Indexing Micellar Polarity. J. Colloid Interface Sci. 2006, 294 (1), 248-254.

(47) Gulati, S.; Jamshad, M.; Knowles, T. J.; Morrison, K. A.; Downing, R.; Cant, N.; Collins, R.; Koenderink, J. B.; Ford, R. C.; Overduin, M.; et al. Detergent-Free Purification of ABC (ATPBinding-Cassette) Transporters. Biochem. J. 2014, 461 (2), 269-278.

(48) Danielczak, B.; Keller, S. Lipid Exchange among Polymer-Encapsulated Nanodiscs by TimeResolved Förster Resonance Energy Transfer. Methods 2020.

(49) Oluwole, A. O.; Klingler, J.; Danielczak, B.; Babalola, J. O.; Vargas, C.; Pabst, G.; Keller, S. Formation of Lipid-Bilayer Nanodiscs by Diisobutylene/Maleic Acid (DIBMA) Copolymer. Langmuir 2017, 33 (50), 14378-14388.

(50) Hazell, G.; Arnold, T.; Barker, R. D.; Clifton, L. A.; Steinke, N. J.; Tognoloni, C.; Edler, K. J. Evidence of Lipid Exchange in Styrene Maleic Acid Lipid Particle (SMALP) Nanodisc Systems. Langmuir 2016, 32 (45), 11845-11853. 
(51) Schmidt, V.; Sturgis, J. N. Modifying Styrene-Maleic Acid Co-Polymer for Studying Lipid Nanodiscs. Biochim. Biophys. Acta - Biomembr. 2018, 1860 (3), 777-783.

(52) Qazi, M. J.; Schlegel, S. J.; Backus, E. H. G.; Bonn, M.; Bonn, D.; Shahidzadeh, N. Dynamic Surface Tension of Surfactants in the Presence of High Salt Concentrations. Langmuir 2020, $36(27), 7956-7964$.

(53) Polyscience. Technical Datasheet https://www.polyscience.eu/smalp/technical-datasheet/ (accessed May 20, 2020).

(54) Kalyanasundaram, K.; Thomas, J. K. Solvent-Dependent Fluorescence of Pyrene-3Carboxaldehyde and Its Applications in the Estimation of Polarity at Micelle-Water Interfaces. J. Phys. Chem. 1977, 81 (23), 2176-2180.

(55) Murphy, P. M. Teaching Structure-Property Relationships: Investigating Molecular Structure and Boiling Point. J. Chem. Educ. 2007, 84 (1), 97-101.

(56) Brechling, A.; Sundermann, M.; Kleineberg, U.; Heinzmann, U. Characterization of DMPC Bilayers and Multilamellar Islands on Hydrophobic Self-Assembled Monolayers of ODS/Si(100) and Mixed ODS-DDS/Si(100). Thin Solid Films 2003, 433 (1-2 SPEC.), 281286.

(57) Postis, V.; Rawson, S.; Mitchell, J. K.; Lee, S. C.; Parslow, R. A.; Dafforn, T. R.; Baldwin, S. A.; Muench, S. P. The Use of SMALPs as a Novel Membrane Protein Scaffold for Structure Study by Negative Stain Electron Microscopy. BBA - Biomembr. 2015, 1848, 496-501.

(58) Parmar, M.; Rawson, S.; Scarff, C. A.; Goldman, A.; Dafforn, T. R.; Muench, S. P.; Postis, V. L. G. Using a SMALP Platform to Determine a Sub-Nm Single Particle Cryo-EM Membrane Protein Structure. Biochim. Biophys. Acta - Biomembr. 2018, 1860 (2), 378-383.

(59) Kantola, A. M.; Lantto, P.; Heinmaa, I.; Vaara, J.; Jokisaari, J. Direct Magnetic-Field Dependence of NMR Chemical Shift. Phys. Chem. Chem. Phys. 2020, 8485-8490.

(60) Lu, Y.; Lin, J.; Wang, L.; Zhang, L.; Cai, C. Self-Assembly of Copolymer Micelles: HigherLevel Assembly for Constructing Hierarchical Structure. Chem. Rev. 2020, No. 120, 4111 4140 .

(61) Hardin, N. Z.; Kocman, V.; Di Mauro, G. M.; Ravula, T.; Ramamoorthy, A. Metal-Chelated Polymer Nanodiscs for NMR Studies. Angew. Chemie - Int. Ed. 2019, 58 (48), 17246-17250. 
(62) Kocman, V.; Di Mauro, G. M.; Veglia, G.; Ramamoorthy, A. Use of Paramagnetic Systems to Speed-up NMR Data Acquisition and for Structural and Dynamic Studies. Solid State Nucl. Magn. Reson. 2019, 102, 36-46. 


\section{Chapter 5}

Spin-Labeled Copolymer Nanodiscs for DNP Studies on Membrane Proteins

The content for this chapter is currently unpublished. 


\subsection{Introduction}

The use of paramagnetic species to enhance NMR spectroscopy sensitivity was discussed in chapters 2 and 3. PRE-NMR relies on the reduction of $\mathrm{T}_{1}$ to speed up the recovery of the magnetization and allowing for shorter recycle delays toward faster data acquisition ${ }^{1}$.

Figure 2.4 in chapter 2 summarizes the three main tagging strategies for PRE studies available in the literature. SMA-EA-DOTA-based nanodiscs, described in chapter 3, offer the fourth strategy. The functionalization of a nanodiscs-forming SMA-based copolymer with chelating units allows for the introduction of paramagnetic tags in the nanodiscs' outer rim. Among the advantages of this approach, must be mentioned (i) the absence of perturbation of membrane protein due to the direct tagging, (ii) the lack of alteration of the native lipid composition retained after direct extraction from the native environment, (iii) avoidance of free paramagnetic ions in solution that might cause unwanted broadening effects and depletion of the quality of the NMR spectrum, thanks to the presence of strong chelators such as DOTA units in the copolymer chains.

At the end of chapter 2, dynamic nuclear polarization coupled to solid-state NMR spectroscopy was treated. DNP-ssNMR has emerged as a prominent research field, offering significant contributions to both materials' science and structural biology ${ }^{2-4}$. The technique improves NMR spectroscopy and relies on paramagnetic species, called polarizing agents, to transfer the large electron spin polarization to nuclear spins upon irradiation with microwaves of an appropriate frequency. Stable organic free radicals such as $\mathrm{TEMPO}^{5,6}$ and $\mathrm{TOTAPOL}^{7-9}$, and complexes of paramagnetic d-block and f-block ions have been successfully employed as polarizing agents ${ }^{10-13}$. Due to their relatively high molecular weight, ssNMR is suitable for studying membrane proteins in phospholipid membranes, providing high-resolution structures.

Moreover, ssNMR can benefit from DNP because, in the right conditions, it can increase the sensitivity up to 10,000 times $^{14}$. Among all the membrane mimetics available, described in chapter I, copolymer-nanodiscs are widely employed in membrane protein studies, and many successes are reported in the literature ${ }^{15,16}$. The SMA-EA-DOTA copolymer, discussed in chapter 3, proposes PRE studies in nanodiscs using solution $\mathrm{NMR}^{17,18}$. The speculation is that SMA-EA-DOTA copolymernanodiscs can be successfully employed in PRE-ssNMR and, of course, to DNP-ssNMR.

Complexes of $\mathrm{Gd}^{3+}$ and $\mathrm{Mn}^{2+}$ ions can be used to enhance ${ }^{13} \mathrm{C}$ NMR spectra via ${ }^{1} \mathrm{H} \mathrm{DNP}{ }^{13}$, although the most employed polarizing agents are organic free radicals ${ }^{8}$. Thus, this chapter proposes two SMA-based copolymers, paramagnetically-tagged with organic free radicals such as TEMPO. ST-10, functionalized with $\sim 10-15 \%$ of TEMPO, and ST-100, fully functionalized with TEMPO units. The synthesis of both copolymers is presented in Figure 5.1. Both can be considered as variants of SMA- 
EA-DOTA with potential applications both in PRE- and DNP-ssNMR studies. The following results are considered preliminary data for a future project that will involve DNP-ssNMR and paramagnetically-labeled nanodisc for the study of membrane proteins.

\subsection{Materials and Methods}

\subsubsection{Reagents and Materials}

Poly(Styrene-co-Maleic Anhydride)-Cumene terminated, SMAnh, $\mathrm{M}_{\mathrm{w}} \sim 1.6 \mathrm{kDa}$, anhydrous 1Methyl-2-pyrrolidone (NMP), 2-Aminoethanol (EA), Diethyl Ether (Et $2 \mathrm{O})$, Hydrochloric Acid $(\mathrm{HCl})$, Sodium Hydroxide $(\mathrm{NaOH})$ were purchased from Sigma-Aldrich ${ }^{\circledR}$ (St. Louis, Missouri). 4amino TEMPO was purchased from Fisher Scientific (Waltham, MA). 1,2-dimyristoyl-sn-glycero-3phosphocholine (DMPC) was purchased from Avanti ${ }^{\circledR}$ Polar Lipids, Inc. (Alabaster, Alabama).

\subsubsection{Polymer synthesis and characterization}

a) Polymers synthesis. SMA-EA copolymer was synthesized, purified, and characterized according to the procedure described in the literature ${ }^{19}$.

ST-10 and ST-100 copolymers were synthesized according to the following procedure, inspired by a similar reaction reported previously ${ }^{17}$. For the synthesis of ST-10, $2 \mathrm{~g}$ of SMAnh, $0.214 \mathrm{~g}$ of 4-amino TEMPO, and $1 \mathrm{~mL}$ of triethylamine were dissolved in $60 \mathrm{~mL}$ of anhydrous NMP and heated to $70{ }^{\circ} \mathrm{C}$ under continuous stirring for 2 hours (Step-1). The addition of an excess of ethanolamine $(\sim 0.5 \mathrm{~mL})$ in the presence of an extra $1 \mathrm{~mL}$ of triethylamine for 2 more hours at the same temperature in the same round bottom flask completed the nucleophilic ring-opening reaction (Step-2). Finally, the product was precipitated using diethyl ether and washed multiple times with deionized water prior to lyophilization. The 2-step reaction yielded about $1.2 \mathrm{~g}$ of ST10 copolymer. Similarly, for the synthesis of ST-100, $2 \mathrm{~g}$ of SMAnh, $1.5 \mathrm{~g}$ of 4-amino TEMPO, and $1 \mathrm{~mL}$ of triethylamine were dissolved in $60 \mathrm{~mL}$ of anhydrous $\mathrm{NMP}$ and heated to $70^{\circ} \mathrm{C}$ under continuous stirring for 4 hours to ensure a complete nucleophilic ring-opening reaction. No ethanolamine was added. Finally, the product was precipitated using diethyl ether and washed multiple times with deionized water prior to lyophilization. The reaction yielded about $1.5 \mathrm{~g}$ of ST-100 copolymer. Both reaction schemes are presented below. 


\section{Synthesis of ST-10}

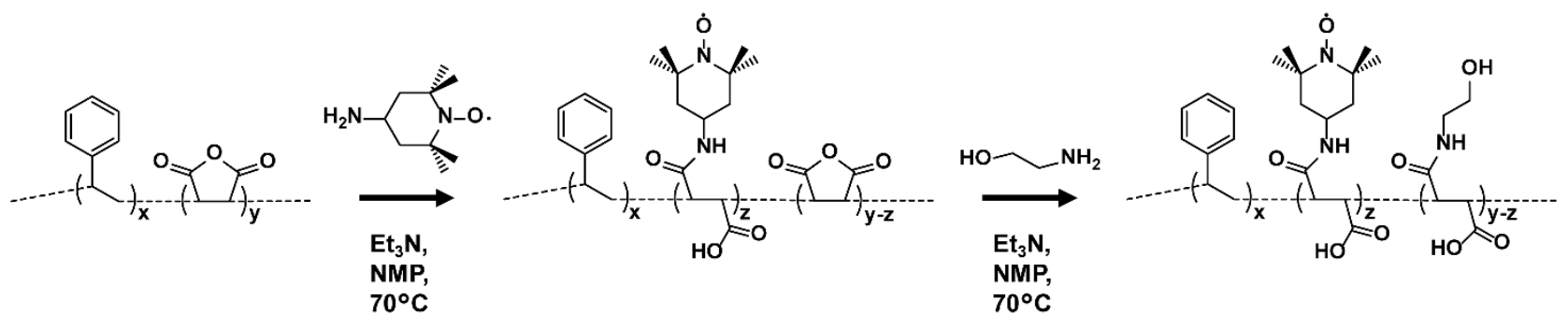

\section{Synthesis of ST-100}
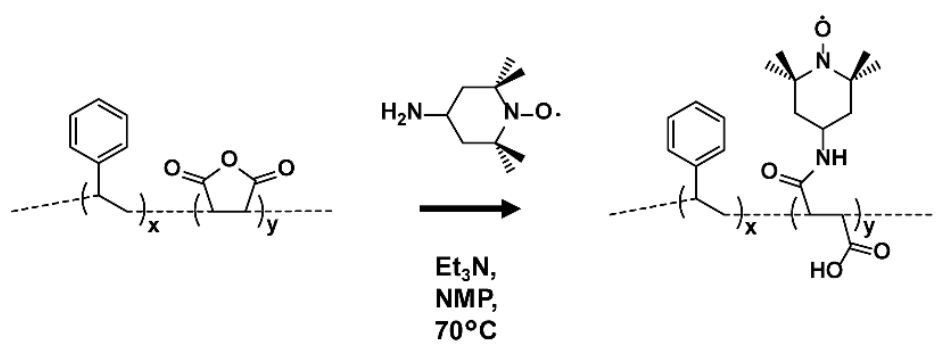

Figure 5.1. Synthesis of ST-10 and ST-100 copolymers. The figure shows the scheme of reaction to obtain both ST-10 and ST-100 copolymers from SMAnh starting material. The synthesis involves a nucleophilic ring-opening reaction. For ST-10, step 1 involves the addition of $\sim 10-15 \%$ of 4 -amino-TEMPO before treatment with an excess of ethanolamine (EA). ST-100 involves a 1-step functionalization with an excess of 4-amino-TEMPO. Both copolymers are synthesized in anhydrous NMP, using triethylamine $\left(\mathrm{Et}_{3} \mathrm{~N}\right)$ as the base.

b) FT-IR Spectroscopy. The newly synthesized polymer was characterized by FT-IR. The carbonyl stretching frequency shift from $1770 \mathrm{~cm}^{-1}$ to $1702 \mathrm{~cm}^{-1}$ indicates the conversion of the anhydride groups to the amide groups, confirming the reaction's success. FT-IR spectra of the starting material SMAnh, SMA-EA, ST-10, and ST-100 are reported below in Figure 5.2. Figure 5.3b reports the expanded $2000-1400 \mathrm{~cm}^{-1}$ region.

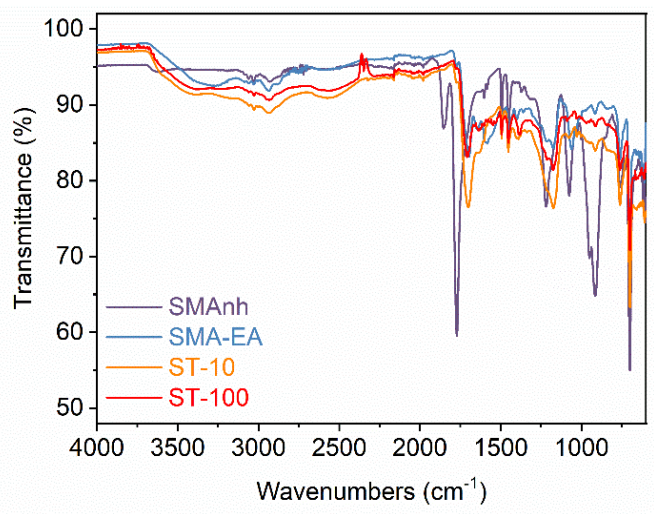

Figure 5.2. Full FT-IR spectra of SMAnh, SMA-EA, ST-10, and ST-100 copolymers. 


\subsubsection{Polymer nanodiscs preparation}

Stock solutions of each copolymer (ST-10 and ST-100) were obtained by dissolving the desired amount of powder in a $0.1 \mathrm{M} \mathrm{NaOH}$ solution. The $\mathrm{pH}$ was then adjusted to 7.4 using $1 \mathrm{M} \mathrm{HCl}$. Polymer-based lipid nanodiscs were prepared by mixing the desired quantity of DMPC lipids and polymers in the ratio $1: 1$ by weight from $20 \mathrm{mg} / \mathrm{mL}$ stock solutions. Each sample was incubated overnight at room temperature before its use.

\subsubsection{Polymer nanodiscs characterization}

a) Static Light Scattering (SLS) - Solubilization Experiments. The SLS experiments were performed using a $4 \mathrm{~mL}$ cuvette (1 cm optical path) under continuous stirring at $25^{\circ} \mathrm{C}$ on a FluoroMax-4® Spectrofluorometer from Horiba Scientific ${ }^{\circledR}$. The excitation wavelength was set at $400 \mathrm{~nm}$, while the emission wavelength was set at $404 \mathrm{~nm}$, and the slit was set to $2 \mathrm{~nm}$. The time-dependent solubilization of MLVs was carried on a $1 \mathrm{mg} / \mathrm{mL}$ suspension of DMPC in 10 $\mathrm{mM}$ HEPES buffer $50 \mathrm{mM} \mathrm{NaCl}$. The intensity of scattered light at a $90^{\circ}$ angle was monitored for 1000 seconds. The solubilization power of both ST-10 and ST-100 copolymers was tested on a solution of DMPC. The amount of polymer added was equivalent (1:1 w/w ratio) for all of them. Data are shown in Figure 5.3c.

b) Electron Paramagnetic Resonance (EPR) Spectroscopy. Samples preparation. ST-10 and ST-100 copolymers were dissolved in $20 \mathrm{mM}$ HEPES buffer, $100 \mathrm{mM} \mathrm{NaCl}, \mathrm{pH} 7$ buffer, brought to a final concentration of $2.5 \%(\mathrm{~m} / \mathrm{v})$, sonicated $\left(30{ }^{\circ} \mathrm{C}\right)$, and vortexed until completely dissolved and the solution was clear. DMPC powdered lipids were dissolved in $20 \mathrm{mM}$ HEPES, $100 \mathrm{mM}$ $\mathrm{NaCl}, \mathrm{pH} 7$ buffer, and brought to a final concentration of $25 \mathrm{mM}$. The lipid slurry was vortexed vigorously for several minutes and underwent at least 20 freeze/sonication cycles $\left(<30{ }^{\circ} \mathrm{C}\right)$. The lipid solution was frozen using liquid nitrogen and stored overnight $\left(-20{ }^{\circ} \mathrm{C}\right)$. The copolymer solution was titrated into the vesicles dropwise until a final weight ratio of 2:1 (polymer:lipid) was achieved, which allowed polymer nanodiscs to form spontaneously.

Continuous wave-electron paramagnetic resonance (CW-EPR) spectroscopy. CW-EPR lineshape analysis verified the stability and functionality of ST-10 and ST-100 copolymers both in solution and in the form of copolymer-nanodiscs. EPR experiments were conducted at the Ohio Advanced EPR Laboratory. CW-EPR spectra of the ST-10 copolymer solution and ST10:DMPC 2:1 w/w nanodiscs were collected at X-band on a Bruker EMX CW-EPR spectrometer 
using an ER041xG microwave bridge and ER4119-HS cavity coupled with a BVT 3000 nitrogen gas temperature controller. Each CW-EPR spectrum was acquired by signal averaging 20-40s field scans with a central field of $3317 \mathrm{G}$ and sweep width of $150 \mathrm{G}$, modulation frequency of $100 \mathrm{kHz}$, modulation amplitude of $1 \mathrm{G}$, and microwave power of $10 \mathrm{~mW}$ at room temperature.

ST-100 polymer solution and ST-100 2:1 nanodiscs were collected using a Bruker EMX nano benchtop CW-EPR spectrometer. Each CW-EPR spectrum was acquired by signal averaging 10, 30 s field scans with a central field of $3420 \mathrm{G}$, and a sweep width of $200 \mathrm{G}$. Data are shown in Figure 5.4.

c) NMR Spectroscopy. Solution NMR experiments were performed at $11.75 \mathrm{~T}$ on a $500 \mathrm{MHz}$ Bruker Avance III HD spectrometer. NMR samples were prepared according to the procedure described in the "Nanodisc Formation," then lyophilized for 24 hours prior to resuspension in $600 \mu \mathrm{L}$ of $\mathrm{D}_{2} \mathrm{O}$ and then transferred to $5 \mathrm{~mm}$ Norrell® Sample Vault Series ${ }^{\mathrm{TM}}$ glass tubes and placed in a commercial $5 \mathrm{~mm}$ quadruple resonance ${ }^{2} \mathrm{H} /{ }^{1} \mathrm{H} /{ }^{15} \mathrm{~N} /{ }^{13} \mathrm{C}$ Bruker round-coil TXITM 500 $\mathrm{SB}$ probe. The experiments were performed in $\mathrm{D}_{2} \mathrm{O}$ at neutral $\mathrm{pH}$ at three different temperatures, 15,25 , and $35^{\circ} \mathrm{C}$. Each sample was made using 1, 2, 4, and $8 \mathrm{mg}$ of lipids, and an equal amount (by weight) of ST-10 and ST-100 polymers, titrated at $\mathrm{pH} 7.4$ to obtain a DMPC:ST-10 and DMPC:ST-100 (1:1 w/w) systems.

- $\quad{ }^{1} \mathrm{H}-\mathrm{NMR} .{ }^{1} \mathrm{H}$ spectra were recorded by collecting 32 scans with a spectral width of $25 \mathrm{ppm}$. The transmitter frequency offset was set at $4.7 \mathrm{ppm}$. Acquisition time and relaxation delay were respectively set at $0.8 \mathrm{~s}$ and $1.0 \mathrm{~s} .{ }^{1} \mathrm{H}-\mathrm{NMR}$ spectra for DMPC:SMA-EA 1:1 (w/w) and DMPC:ST-10 and DMPC:ST-100 1:1 (w/w) are shown in Figure 5.5.

- Inversion recovery experiment. To measure $T_{1}$, an inversion recovery experiment was performed. Fifteen data points from $0.001 \mathrm{~s}$ to $10.0 \mathrm{~s}$ were collected by acquiring 8 scans with a spectral width of $10 \mathrm{ppm}$. The transmitter frequency offset was set at $4.7 \mathrm{ppm}$. Acquisition time and relaxation delay were respectively set at $0.001 \mathrm{~s}$ and $10 \mathrm{~s}$. Data are shown in Figures 5.6-5.8 and Tables 5.1-5.3.

- Data Processing. Data have been processed using both Bruker Topspin ${ }^{\text {TM }} 3.2$ and Mestrelab Research S.L. MestReNova ${ }^{\mathrm{TM}}$ software was used to integrate the peaks of interest. 


\subsection{Results and discussion}

Unlike SMA-EA-DOTA, where the concentration of paramagnetic centers depends on the concentration of the added metal ions and not on the introduced chelating units, TEMPOfunctionalized copolymers do not allow the same fine control. Thus, the decision to synthesize two different formulations and investigate how the introduction of different concentrations of organic free radicals in the copolymer chains affects the ability to form nanodiscs and paramagnetic features.

ST-10 and ST-100 respectively functionalized with $\sim 10 \%$ and $\sim 100 \%$-amino TEMPO, were synthesized using the same starting material (SMAnh, $\sim 1.6 \mathrm{kDa}, \sim 1.3: 1$ styrene-to-maleic anhydride molar ratio) used in the Ramamoorthy research group to obtain all the copolymers, from SMA-EA ${ }^{20}$ to SMA-EA-DOTA ${ }^{17,18}$, discussed in the second part of chapter 1 . The synthetic strategy is detailed in the Materials and Methods paragraph above, and the reaction scheme is reported in Figure 5.1. Figure 5.3a below shows the structures of the newly synthesized ST-10 and ST-100 in comparison with the structures of the starting material and SMA-EA, here used as a non-paramagnetic counterpart. FT-IR spectra show the disappearance of the maleic anhydride ring in favor of amide bonds (Figure 5.2 and 5.3b). Both ST-10 and ST-100 copolymers successfully solubilized DMPC MLVs at room temperature $\left(\sim 25^{\circ} \mathrm{C}\right)$, as reported in Figure 5.3c.

a)

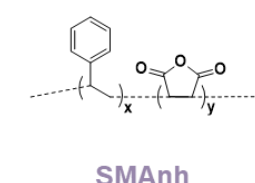

SMAnh

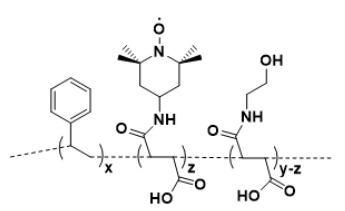

ST-10

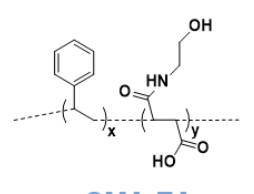

SMA-EA

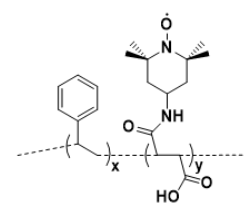

ST-100 b)

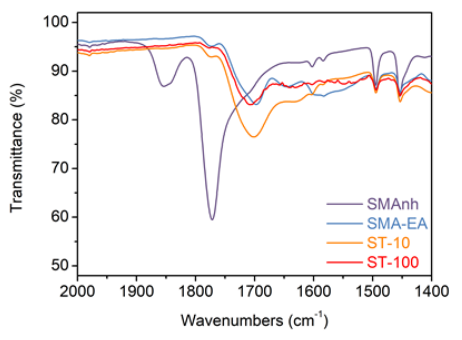

c)

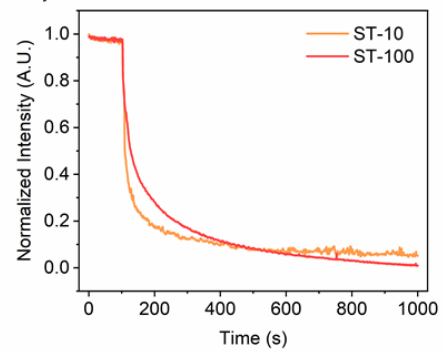

Figure 5.3. Characterization of polymers and polymer nanodiscs. a) Shows the structures of the SMAnh (the starting material), SMA-EA, ST-10, and ST-100 copolymers. b) FT-IR spectra of the starting material (SMAnh - purple), SMAEA (light blue), ST-10 (orange), and ST-100 (red). FT-IR results confirm the functionalization of the SMAnh and show similarities among SMA-EA, ST-10, and ST-100. Full spectra are presented in Figure 5.2. 
Figure 5.4 shows a comparison of the CW-EPR lineshapes for solutions of the two copolymers (on the left side, continuous line) and solutions of copolymer-nanodiscs (on the right side, dashed line). Solutions of ST-10 copolymer are shown in orange, while solutions of ST-100 copolymer are in red. Among solutions of ST-10 and ST-100 micellar copolymers, lineshapes are similar to each other and resemble the lineshape for a stable free radical in solution ${ }^{21}$. Thus, these preliminary data confirm the free radicals' stability and functionality (TEMPO units) in the chains of both spin-labeled copolymers.

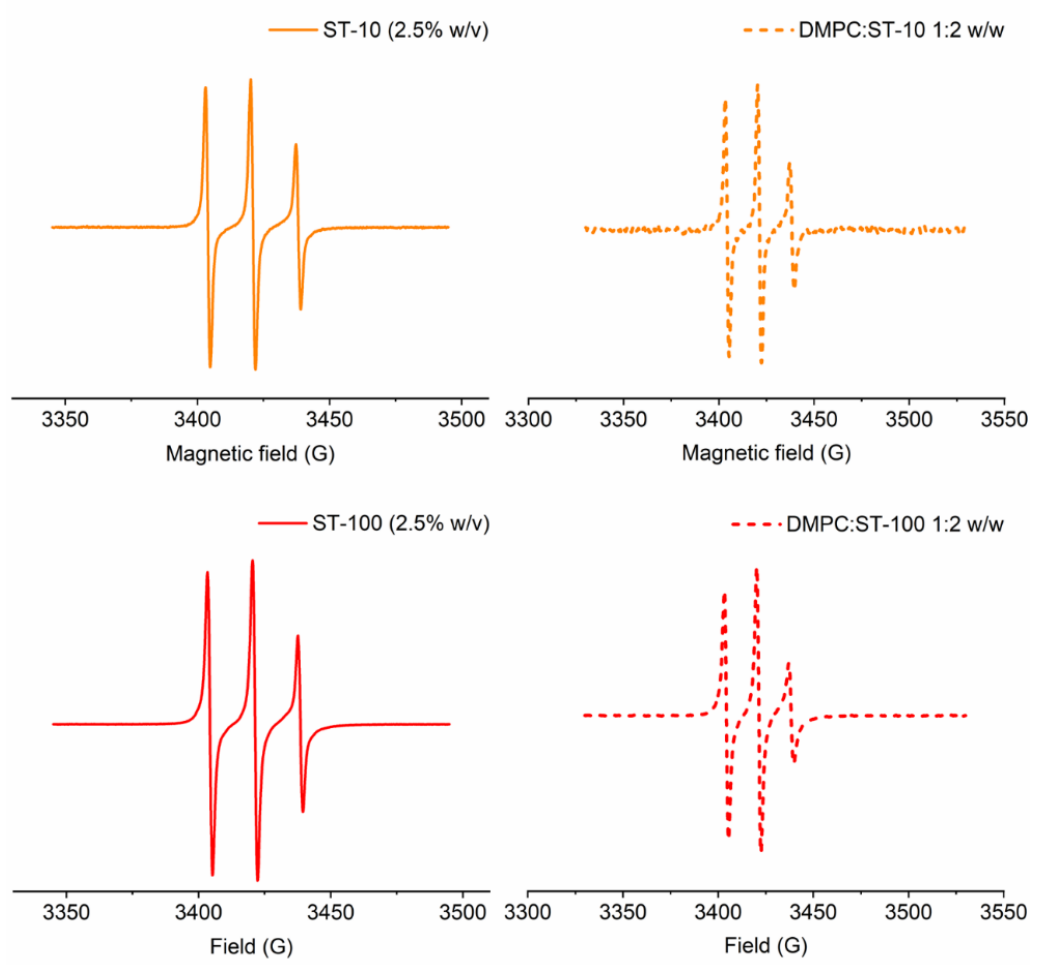

Figure 5.4. CW-EPR spectra of copolymers and copolymer nanodiscs. The top panels show data from a solution of ST-10 (top-left panel) and nanodiscs with DMPC in a 2:1 w/w ratio (top-right panel). The bottom panels show a solution of ST-100 (bottom-left panel) and ST-100:DMPC nanodiscs in a 2:1 w/w ratio (bottom-right panel).

Both spectra from the nanodiscs solutions of ST-10:DMPC 2:1 w/w and ST-100:DMPC 2:1 w/w are comparable to each other. Interestingly, nanodiscs samples are similar to the free copolymers in solution, and no broadening is observed. The study of CW-EPR spectroscopy lineshape of the copolymers' solutions and copolymers nanodiscs was carried out to establish the radical groups' functionality. However, further investigations are needed to shed light on both the structural and dynamic of spin-labeled copolymer-nanodiscs. These preliminary results are essential, as the TEMPO groups' functionality in both copolymers' solution and copolymer-nanodiscs' solutions must be maintained. The spin-labels' functionality is retained when the polymer is alone in solution or combined with lipids. This serves as a benchmark for numerous projects. For example, the incorporation of membrane proteins can allow a better understanding of membrane protein dynamics. 
CW-EPR and pulsed EPR spectroscopies may benefit from spin-labeled nanodiscs-forming copolymers. For example, chapter 4 concludes by speculating about the possibility of copolymer removal by dilution. Quantitative uses of EPR spectroscopy are reported in the literature ${ }^{22-24}$, but the differentiation among copolymer chains bound to the nanodiscs and free in solution might be challenging. However, in combination with other techniques, EPR spectroscopy can shed light on that equilibrium, validating what speculated.

Once verified the presence of functional spin-labels on both copolymers and copolymer-nanodiscs solutions, PRE effects in solution NMR were tested on ST-10:DMPC and ST100:DMPC, both 1:1 (w/w), nanodiscs to confront the paramagnetic features of both copolymers with the previously reported SMA-EA-DOTA in the presence of different concentrations of several paramagnetic ions. The ${ }^{1} \mathrm{H}$ NMR peaks were observed in the copolymer-lipid nanodiscs spectra. The complete assignment is done accordingly to previous reports in the literature ${ }^{17,18,25}$.
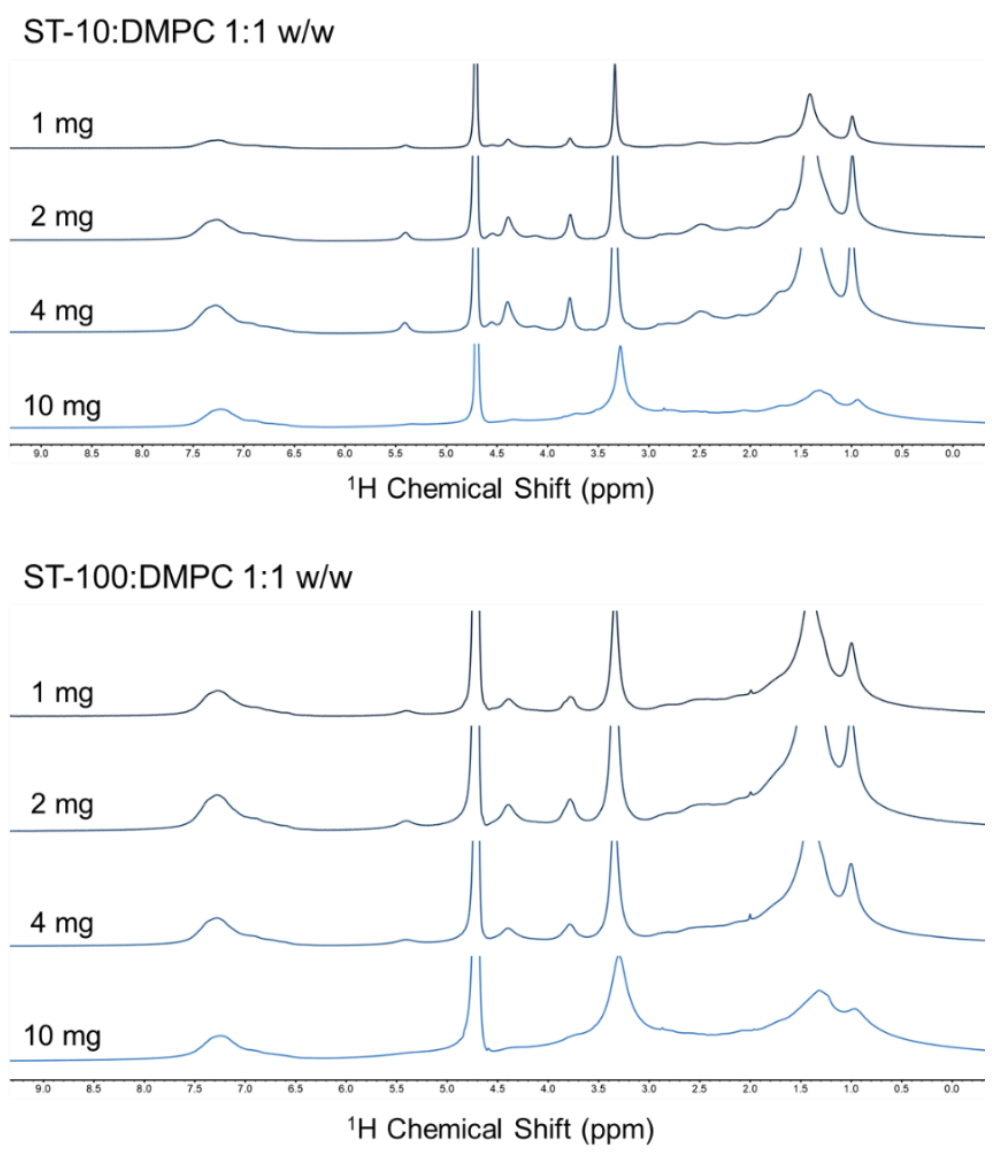

Figure 5.5. ${ }^{1} \mathrm{H}$ NMR spectra of paramagnetically labeled nanodiscs varying the free radical concentration. a) shows ${ }^{1} \mathrm{H}$ spectra of 1, 2, 4, and $10 \mathrm{mg} \mathrm{ST}-10: D M P C(1: 1 \mathrm{w} / \mathrm{w})$ nanodiscs. Significant broadening effects are observed for the $10 \mathrm{mg}$ sample. b) shows the ${ }^{1} \mathrm{H}$ spectra of 1,2 , 4, and $10 \mathrm{mg}$ of ST-100:DMPC $(1: 1 \mathrm{w} / \mathrm{w})$ nanodiscs. Due to the larger concentration of free radicals in ST-100, broadening effects are more prominent, starting from lower copolymer concentrations. 
Figure 5.5 shows ${ }^{1} \mathrm{H}$ solution NMR spectra of 1, 2, 4, and $10 \mathrm{mg}$ of ST-10:DMPC (1:1 w/w), and ST100:DMPC (1:1 w/w) nanodiscs. As expected, both copolymers' increasing concentration increases the free radicals' concentration in the sample, leading to both $T_{1}$ and $T_{2}$ shortening. As observed in Figure 5.5, samples containing $10 \mathrm{mg}$ of ST-10 show significant peaks broadening, and this effect is notably visible in the region 3.5-4.5 ppm, associated with the protons $\alpha$ and $\beta$ from the phospholipids' glycerol moiety. Moreover, because of the larger content of free radicals, ST-100 shows broadening effects starting from $4 \mathrm{mg}$ of the copolymer in the sample.

As discussed in chapter 3 , the $\mathrm{T}_{1}$-reduction, consequence of the paramagnetic effects due to TEMPO units' introduction, was investigated as follows. Four peaks, both from lipids and copolymers, were considered, recording how the $\mathrm{T}_{1}$ is affected in different regions of the nanodiscs - core vs. rim vs. surface. As previously reported ${ }^{17,18}$, the peak at $\sim 1 \mathrm{ppm}$ is associated with the protons from the lipid terminal methyl group $\left(-\mathrm{CH}_{3}\right)$, while the one at $\sim 1.4 \mathrm{ppm}$ from the protons in the methylene groups of the lipid acyl chains $\left(-\mathrm{CH}_{2}-\right.$ from $\mathrm{C} 4$ to $\left.\mathrm{C} 13\right)$. Both are related to the core of the nanoparticle. The peak at $\sim 3.3 \mathrm{ppm}$ originated by the protons from the quaternary ammonium groups $\left(\left(-\mathrm{CH}_{3}\right)_{3}, \gamma\right)$ is associated with the nanodiscs' surface. In comparison, the peak at $\sim 7.3 \mathrm{ppm}$, which originated from the protons in the aromatic ring from the copolymer's styrene fraction, is associated with the outer rim of the nanoparticle ${ }^{17,18}$.

As reported in the literature ${ }^{1,17,18}$ and detailed in chapters 2 and 3, the inversion-recovery NMR experiments were performed to measure the $T_{1}$ values of the protons mentioned above to examine the effect of different concentrations on free radicals in the copolymer chains. The $\mathrm{T}_{1}$ values were obtained by integrating the ${ }^{1} \mathrm{H}$ NMR peak of interest and then fitting the experimental results according to equation 1 in chapter 3 .

The resulting $T_{1}$ values are reported in the following tables (Tables 5.1-3), while experimental data and fitting are reported in Figure 5.6 and 5.7. Table 1 shows the experimental $\mathrm{T}_{1}$ values obtained for the diamagnetic SMA-EA:DMPC $(1: 1 \mathrm{w} / \mathrm{w})$ nanodiscs. Results are consistent with previously reported data ${ }^{18}$ (shown in Figure 3.9, chapter 3) among various nanodiscs concentrations.

\begin{tabular}{c|cccc} 
SMA-EA:DMPC $(\mathbf{1 : 1} \mathbf{w} / \mathbf{w})$ & Aromatic & Gamma & C4-C13 & C14 \\
\hline $\mathbf{1 ~ m g}$ & $1.70 \mathrm{~s}$ & $0.58 \mathrm{~s}$ & $0.74 \mathrm{~s}$ & $0.80 \mathrm{~s}$ \\
$\mathbf{2} \mathbf{~ m g}$ & $1.70 \mathrm{~s}$ & $0.58 \mathrm{~s}$ & $0.74 \mathrm{~s}$ & $0.78 \mathrm{~s}$ \\
$\mathbf{4} \mathbf{~ m g}$ & $1.70 \mathrm{~s}$ & $0.58 \mathrm{~s}$ & $0.74 \mathrm{~s}$ & $0.78 \mathrm{~s}$ \\
\hline
\end{tabular}

Table 5.1. T 1 values for SMA-EA:DMPC $(1: 1 \mathrm{w} / \mathrm{w})$ nanodiscs varying the free radicals' concentration in the sample. Highlighted in gray is the condition used in the experiments shown in chapter 3. 

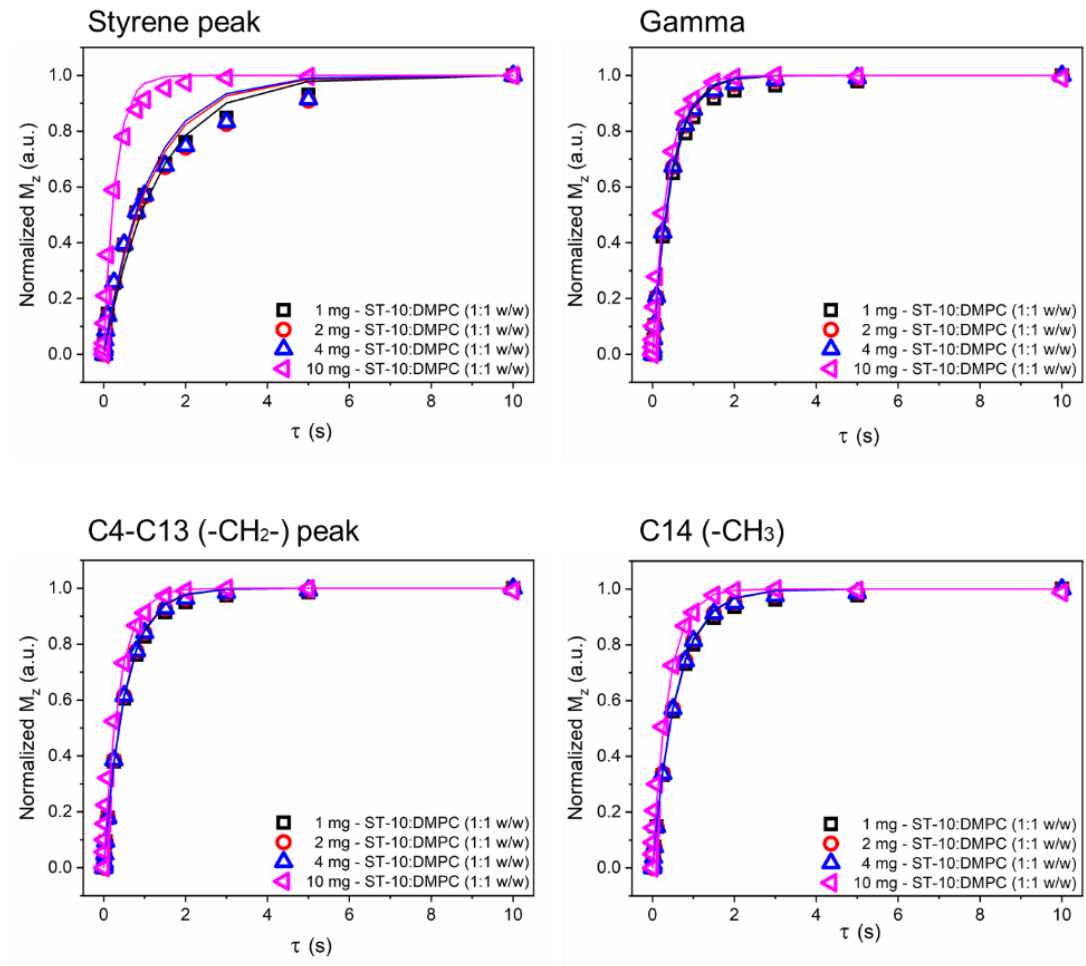

ST-100:DMPC 1:1 (w/w)
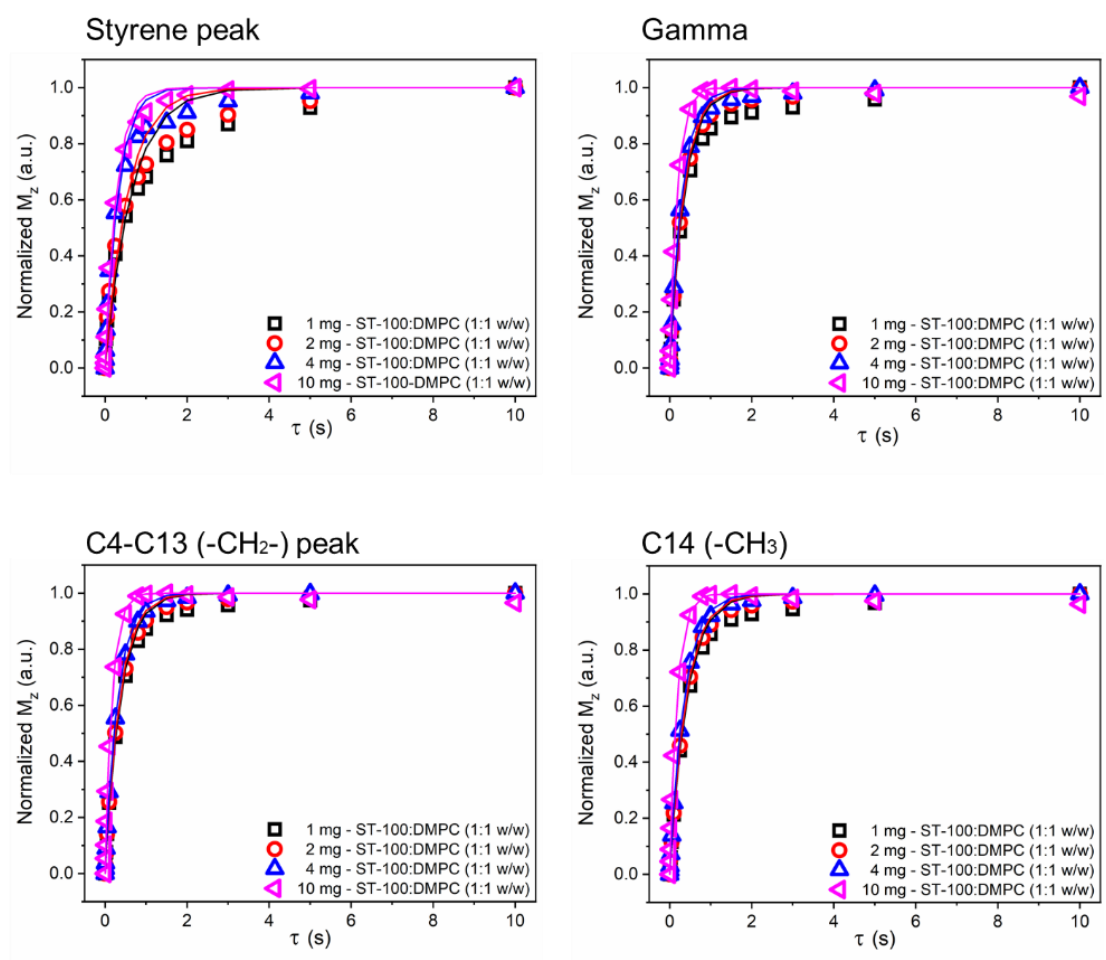

Figure 5.6. Measurement of $\mathbf{T}_{1}$ for protons. The figure shows the experimental data obtained inversion recovery NMR experiments for 1:1 w/w DMPC:ST-10 nanodiscs (top) and 1:1 w/w DMPC:ST-100 nanodiscs (bottom) at $35^{\circ} \mathrm{C}$ for both to determine $T_{1}$ values of protons varying the free radicals concentrations as indicated. Equation 1 from Chapter 3 was used to obtain the best-fitting values. 
Table 5.2 contains the $\mathrm{T}_{1}$ data obtained from ST-10:DMPC 1:1 (w/w) nanodiscs. Despite the lipidto-polymer weight ratio is constant, as expected, by increasing the concentration of polymer in the sample, the concentration of free radicals increases; thus, the $\mathrm{T}_{1}$ is reduced as reported. From $1 \mathrm{mg}$ to $4 \mathrm{mg}$, the reduction is about 15\%. Nevertheless, comparing $4 \mathrm{mg}$ of ST-10 with $4 \mathrm{mg}$ of SMA-EA, the $\mathrm{T}_{1}$ is averagely reduced by $\sim 28 \%$ in the selected peaks compared to the diamagnetic counterpart. To confirm the trend, a sample of $10 \mathrm{mg}$ ST-10:DMPC 1:1 (w/w) was studied. In this case, the data reported for $\mathrm{C} 4-\mathrm{C} 13$ and $\mathrm{C} 14$ (labeled with an asterisk, ${ }^{*}$ ) are not fully reliable due to significant broadening effects $\left(\mathrm{T}_{2}\right.$ shortening).

\begin{tabular}{c|cccc} 
ST-10:DMPC (1:1 w/w) & Aromatic & Gamma & C4-C13 & C14 \\
\hline $\mathbf{1 ~} \mathbf{~ m g}$ & $1.3 \mathrm{~s}$ & $0.46 \mathrm{~s}$ & $0.53 \mathrm{~s}$ & $0.59 \mathrm{~s}$ \\
$\mathbf{2} \mathbf{~ m g}$ & $1.2 \mathrm{~s}$ & $0.44 \mathrm{~s}$ & $0.52 \mathrm{~s}$ & $0.58 \mathrm{~s}$ \\
$\mathbf{4} \mathbf{~ m g}$ & $1.1 \mathrm{~s}$ & $0.44 \mathrm{~s}$ & $0.52 \mathrm{~s}$ & $0.58 \mathrm{~s}$ \\
$\mathbf{1 0} \mathbf{~ m g}$ & $0.74 \mathrm{~s}$ & $0.37 \mathrm{~s}$ & $0.37 \mathrm{~s}^{*}$ & $0.38 \mathrm{~s}^{*}$ \\
\hline
\end{tabular}

Table 5.2. $T_{1}$ values for $S T-10: D M P C(1: 1 \mathrm{w} / \mathrm{w})$ nanodiscs varying the free radicals' concentration in the sample. Highlighted in gray is the condition used in the experiments shown in chapter 3 . The values labeled with the asterisks come from significantly broad peaks and not entirely reliable. Figure 5.5 shows the associated ${ }^{1} \mathrm{H}-\mathrm{NMR}$ spectrum.

Finally, the $\mathrm{T}_{1}$ values of ST-100:DMPC 1:1 (w/w) nanodiscs at different concentrations are reported in Table 3. Compared to ST-10:DMPC 1:1 (w/w) nanodiscs, the drop is more prominent when passing from $1 \mathrm{mg}$ to $10 \mathrm{mg}$. Furthermore, significant is the reduction with the diamagnetic counterpart. On average, the $\mathrm{T}_{1}$-reduction between SMA-EA and ST-100-based nanodiscs is of about $50 \%$ and beyond. 

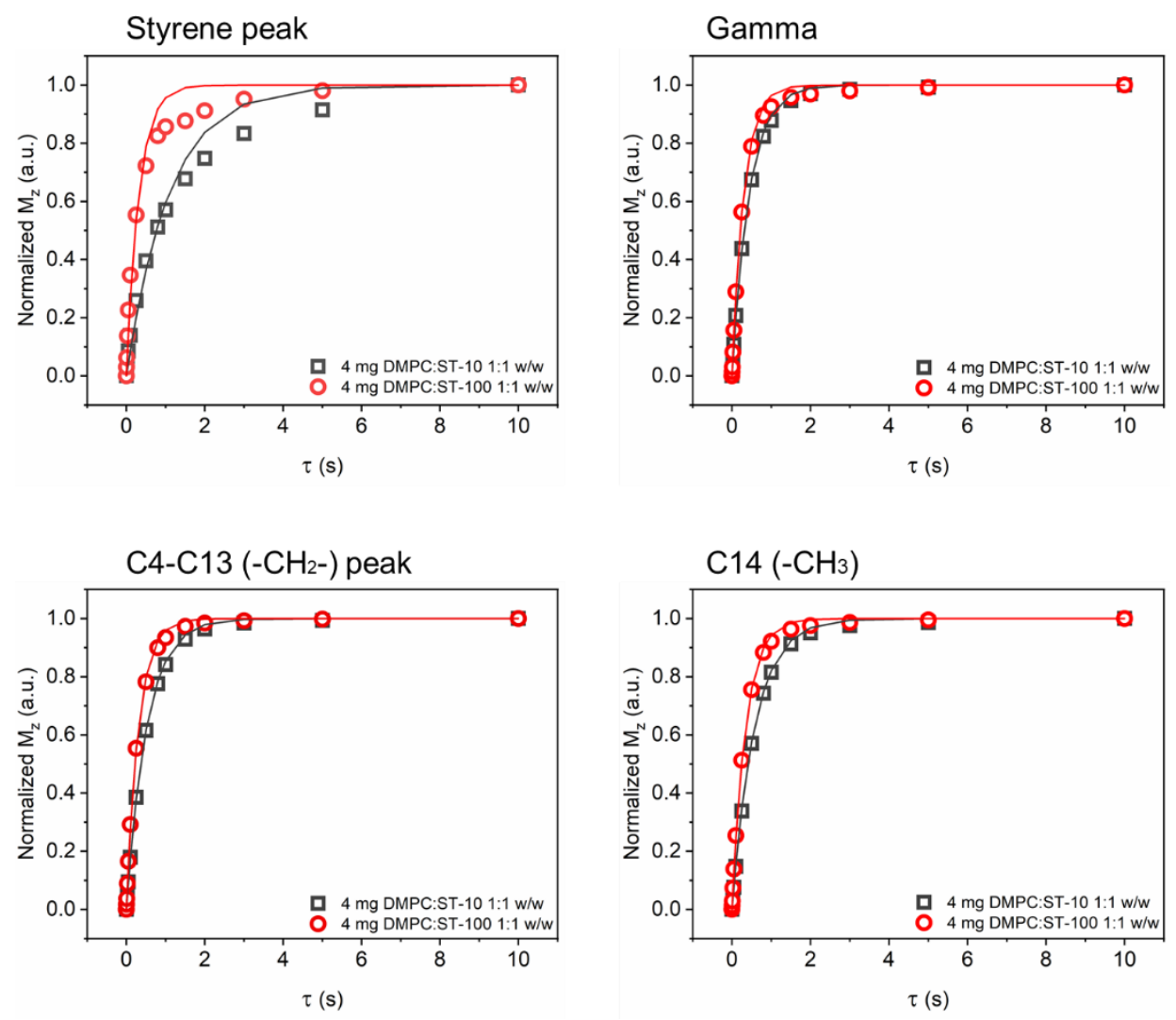

Figure 5.7. A comparison of $\mathrm{T}_{1}$ for protons of ST-10 and ST-100 based nanodiscs. Spin-inversion NMR experimental data obtained from $4 \mathrm{mg}$ 1:1 w/w ST-10:DMPC and ST-100:DMPC nanodiscs to determine $\mathrm{T}_{1}$ values of protons. Equation 1 from chapter 3 was used to obtain the best-fitting values given in Tables 1-3, chapter 5 .

Unfortunately, ST-100 leads to significant line broadening, as reported in Figure 5.8.

\begin{tabular}{c|cccc} 
ST-100:DMPC (1:1 w/w) & Aromatic & Gamma & C4-C13 & C14 \\
\hline $\mathbf{1 ~} \mathbf{~ g g}$ & $0.65 \mathrm{~s}$ & $0.36 \mathrm{~s}$ & $0.38 \mathrm{~s}$ & $0.42 \mathrm{~s}$ \\
$\mathbf{2} \mathbf{~ m g}$ & $0.56 \mathrm{~s}$ & $0.34 \mathrm{~s}$ & $0.36 \mathrm{~s}$ & $0.40 \mathrm{~s}$ \\
$\mathbf{4} \mathbf{~} \mathbf{g}$ & $0.32 \mathrm{~s}$ & $0.30 \mathrm{~s}$ & $0.31 \mathrm{~s}$ & $0.35 \mathrm{~s}$ \\
$\mathbf{1 0} \mathbf{~ m g}$ & $0.28 \mathrm{~s}$ & $0.18 \mathrm{~s}$ & $0.17 \mathrm{~s}^{*}$ & $0.18 \mathrm{~s}^{*}$ \\
\hline
\end{tabular}

Table 5.3. $T_{1}$ values for ST-100:DMPC (1:1 w/w) nanodiscs varying the free radicals' concentration in the sample. Highlighted in gray is the condition used in the experiments shown in chapter 3 . The values labeled with the asterisks come from significantly broad peaks and not entirely reliable. Figure 5.5 shows the associated ${ }^{1} \mathrm{H}-\mathrm{NMR}$ spectrum.

Figure 5.8 shows the comparison of the $\mathrm{T}_{1}$ values of diamagnetic SMA-EA, SMA-EA-DOTA (loaded with $0.5 \mathrm{mM} \mathrm{Gd}^{3+}$ ions), ST-10, and ST-100 nanodiscs. All the reported systems contain $4 \mathrm{mg}$ of lipids and a 1:1 w/w lipid-to-copolymer ratio. As expected, all the paramagnetic systems show a reduction in $\mathrm{T}_{1}$ values. 
SMA-EA-DOTA, ST-10, and ST-100 copolymers shorten $\mathrm{T}_{1}$ times of different ${ }^{1} \mathrm{H}$ peaks in the nanodiscs in a unique way. In chapter 3, SMA-EA-DOTA copolymer loaded with $\mathrm{Gd}^{3+}$ ions showed overall the best performances in terms of $\mathrm{T}_{1}$ reduction if compared to other paramagnetic metals. $\mathrm{T}_{1}$ values for $\mathrm{Gd}^{3+}$-loaded SMA-EA-DOTA nanodiscs are replotted in Figure 5.8 and compared with ST-10 and ST-100 in similar conditions. On the one hand, it is noticeable how the reduction is incredibly efficient for the aromatic and gamma peaks for SMA-EA-DOTA.

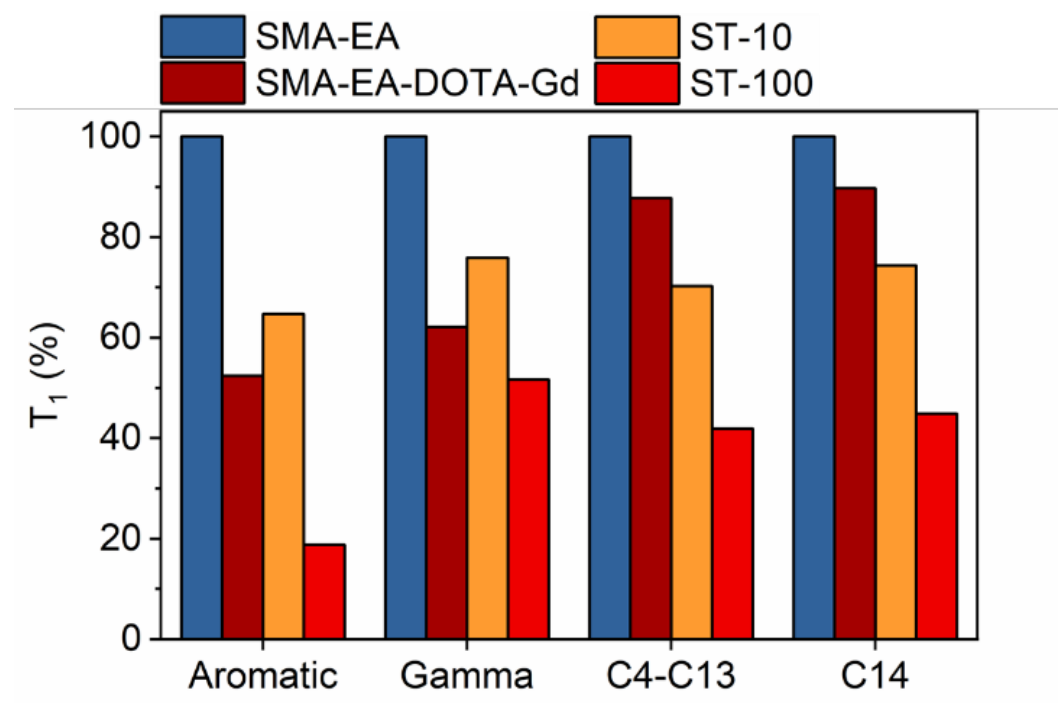

Figure 5.8. Efficiency in paramagnetic labeling of copolymer nanodiscs. Graphical comparison of the $T_{1}$ values reduction in the $1: 1 \mathrm{w} / \mathrm{w}$ copolymer-lipid nanodiscs reported in this thesis. SMA-EA-DOTA loaded with $\left[\mathrm{Gd}^{3+}\right]=0.5$ $\mathrm{mM}$ (reported in chapter 3), $4 \mathrm{mg}$ of ST-10, and $4 \mathrm{mg}$ of ST-100 all show $\mathrm{T}_{1}$ reduction because of the presence of paramagnetic labels in the outer rim of the copolymer-nanodiscs. Values are expressed in percentages for the diamagnetic SMA-EA copolymer ( $\left.\mathrm{T}_{1} 100 \%\right)$.

On the other hand, ST-10 showed more efficiency in reducing the $\mathrm{T}_{1}$ values in the core of the nanoparticle (phospholipid acyl chains or $\mathrm{C} 4-\mathrm{C} 13$ and $\mathrm{C} 14$-associated protons). The speculation is tied to the chemical differences between DOTA and TEMPO units. In fact, DOTA is more hydrophilic than TEMPO; thus, it is legit to believe that DOTA units are more exposed to the surrounding solution, while TEMPO units are more embedded in the hydrophobic core of the nanodiscs. The high content of free radicals makes ST-100 able to reduce $\mathrm{T}_{1}$ for all the considered peaks by $\sim 50 \%$ and more in the same concentrations of nanodiscs and temperature conditions. Further investigations can confirm such speculation and offer better insights into the systems here described. 


\subsection{Conclusions}

SMA-based copolymers have proved their efficiency in the direct extraction of membrane proteins from their native environment, and copolymer-nanodiscs have shown their vast potential as membrane mimetics. Ravula et al. demonstrated that short-chained SMA-based copolymers overcome some of the limitations that affect the early formulations of SMA copolymers ${ }^{17,20,26,27}$. To expand copolymer-nanodiscs applications toward NMR spectroscopy, SMA-EA-DOTA allows PRENMR studies in nanodiscs, offering a less invasive approach toward the preservation of the integrity of the sample, if compared with the alternatives discussed in chapter $2^{1}$. In fact, SMA-EA-DOTAnanodiscs require neither paramagnetic labels on the membrane protein nor the use of paramagnetically-labeled phospholipids. The strong chelating units in the copolymer chains avoid free metals in solution, forcing the paramagnetic sources on the outer rim of the nanodiscs. Thus, SMA-EA-DOTA allows an efficient $\mathrm{T}_{1}$-reduction with minimum-to-no alteration of the spectral quality due to unwanted $\mathrm{T}_{2}$-reduction ${ }^{17,18}$.

In this chapter, ST-10 and ST-100 copolymers are shown to dissolve MLVs of DMPC successfully. The presence of stable organic free radicals on the nanodiscs' outer rim reduces the $\mathrm{T}_{1}$-values as expected. However, the nature of both copolymers makes them less versatile if compared with SMAEA-DOTA. In fact, the paramagnetic properties of SMA-EA-DOTA depend on the concentration of the added paramagnetic ion, of ease control. In contrast, for ST-10 and ST-100, these properties depend on (i) the percentage of functionalization on the copolymer, (ii) the amount of active free radicals on the copolymer chains, (iii) the concentration of the copolymer in the sample.

Further studies might elucidate what the most efficient way of using ST-10 or ST-100 copolymers is. For example, it can be investigated whether it is more convenient to intervene in the final formulation of the copolymer, i.e., by synthesizing new ST-Xs copolymers or whether it is more efficient to work on the final formulation of the copolymer-nanodiscs by merely using mixed copolymers as a consequence of the speculations in chapter 4. Combined with other techniques, they might offer quantitative estimations of the equilibrium nanodiscs-bound-free in solution copolymer chains, discussed in chapter 4. Finally, ST-10 and ST-100 might represent a valid alternative to TOTAPOL and other biradicals in DNP-ssNMR studies on membrane proteins. 


\section{Acknowledgements}

This research was supported by the National Institute of Health (GM084018 to AR). I would like to thank Dr. Nathaniel Hardin for the discussions in designing the synthesis of the copolymers and a special thanks to Benjamin D. Hardin for his contribution collecting the data from the CW-EPR experiments in the laboratory of Gary Lorigan at Miami University $(\mathrm{OH})$. 


\section{References}

(1) Kocman, V.; Di Mauro, G. M.; Veglia, G.; Ramamoorthy, A. Use of Paramagnetic Systems to Speed-up NMR Data Acquisition and for Structural and Dynamic Studies. Solid State Nucl. Magn. Reson. 2019, 102, 36-46.

(2) Akbey, Ü.; Oschkinat, H. Structural Biology Applications of Solid State MAS DNP NMR. 2016.

(3) Rosay, M.; Lansing, J. C.; Haddad, K. C.; Bachovchin, W. W.; Herzfeld, J.; Temkin, R. J.; Griffin, R. G. High-Frequency Dynamic Nuclear Polarization in MAS Spectra of Membrane and Soluble Proteins. J. Am. Chem. Soc. 2003, 125 (45), 13626-13627.

(4) Good, D. B.; Voinov, M. A.; Bolton, D.; Ward, M. E.; Sergeyev, I. V.; Caporini, M.; Scheffer, P.; Lo, A.; Rosay, M.; Marek, A.; et al. A Biradical-Tagged Phospholipid as a Polarizing Agent for Solid-State MAS Dynamic Nuclear Polarization NMR of Membrane Proteins. Solid State Nucl. Magn. Reson. 2019, 100, 92-101.

(5) Maly, T.; Debelouchina, G. T.; Bajaj, V. S.; Hu, K.-N.; Joo, C.-G.; Mak-Jurkauskas, M. L.; Sirigiri, J. R.; Van Der Wel, P. C. A.; Herzfeld, J.; Temkin, R. J.; et al. High-Frequency Dynamic Nuclear Polarization Using Mixtures of TEMPO and Trityl Radicals. Cit. J. Chem. Phys. 2008, 128, 15101.

(6) Gerfen, G. J.; Becerra, L. R.; Hall, D. A.; Griffin, R. G.; Temkin, R. J.; Singel, D. J. High Frequency $(140 \mathrm{GHz})$ Dynamic Nuclear Polarization: Polarization Transfer to a Solute in Frozen Aqueous Solution. J. Chem. Phys. 1995, 102 (24), 9494-9497.

(7) Song, C.; Hu, K. N.; Joo, C. G.; Swager, T. M.; Griffin, R. G. TOTAPOL: A Biradical Polarizing Agent for Dynamic Nuclear Polarization Experiments in Aqueous Media. J. Am. Chem. Soc. 2006, 128 (35), 11385-11390.

(8) Sauvée, C.; Rosay, M.; Casano, G.; Aussenac, F.; Weber, R. T.; Ouari, O.; Tordo, P. Highly 
Efficient, Water-Soluble Polarizing Agents for Dynamic Nuclear Polarization at High Frequency. Angew. Chemie - Int. Ed. 2013, 52 (41), 10858-10861.

(9) Kubicki, D. J.; Casano, G.; Schwarzwälder, M.; Abel, S.; Sauvée, C.; Ganesan, K.; Yulikov, M.; Rossini, A. J.; Jeschke, G.; Copéret, C.; et al. Rational Design of Dinitroxide Biradicals for Efficient Cross-Effect Dynamic Nuclear Polarization. Chem. Sci. 2016, 7 (1), 550-558.

(10) Kaushik, M.; Qi, M.; Godt, A.; Corzilius, B. Bis-Gadolinium Complexes for Solid Effect and Cross Effect Dynamic Nuclear Polarization. Angew. Chemie - Int. Ed. 2017, 56 (15), 42954299.

(11) Gordon, J. W.; Fain, S. B.; Rowland, I. J. Effect of Lanthanide Ions on Dynamic Nuclear Polarization Enhancement and Liquid-State T1 Relaxation. Magn. Reson. Med. 2012, 68 (6), 1949-1954.

(12) Niedbalski, P.; Parish, C.; Wang, Q.; Kiswandhi, A.; Hayati, Z.; Song, L.; Lumata, L. 13C Dynamic Nuclear Polarization Using a Trimeric $\mathrm{Gd}^{3+}$ Complex as an Additive. J. Phys. Chem. A 2017, 121 (27), 5127-5135.

(13) Kaushik, M.; Bahrenberg, T.; Can, T. V; Caporini, M. A.; Silvers, R.; Heiliger, J.; Smith, A. A.; Schwalbe, H.; Griffin, R. G.; Corzilius, B. Gd(III) and Mn(II) Complexes for Dynamic Nuclear Polarization: Small Molecular Chelate Polarizing Agents and Applications with SiteDirected Spin Labeling of Proteins. Phys. Chem. Chem. Phys 2016, 18, 27205-27218.

(14) Ardenkjær-Larsen, J. H.; Fridlund, B.; Gram, A.; Hansson, G.; Hansson, L.; Lerche, M. H.; Servin, R.; Thaning, M.; Golman, K. Increase in Signal-to-Noise Ratio of >10,000 Times in Liquid-State NMR. Proc. Natl. Acad. Sci. U. S. A. 2003, 100 (18), 10158-10163.

(15) Ravula, T.; Ramamoorthy, A. Magnetic Alignment of Polymer Macro-Nanodiscs Enables Residual-Dipolar-Coupling-Based High-Resolution Structural Studies by NMR Spectroscopy. Angew. Chemie - Int. Ed. 2019, 58 (42), 14925-14928.

(16) Ravula, T.; Hardin, N. Z.; Bai, J.; Im, S. C.; Waskell, L.; Ramamoorthy, A. Effect of Polymer Charge on Functional Reconstitution of Membrane Proteins in Polymer Nanodiscs. Chem. Commun. 2018, 54 (69), 9615-9618.

(17) Hardin, N. Z.; Kocman, V.; Di Mauro, G. M.; Ravula, T.; Ramamoorthy, A. Metal-Chelated Polymer Nanodiscs for NMR Studies. Angew. Chemie - Int. Ed. 2019, 58 (48), 17246-17250.

(18) Di Mauro, G. M.; Hardin, N. Z.; Ramamoorthy, A. Lipid-Nanodiscs Formed by Paramagnetic 
Metal Chelated Polymer for Fast NMR Data Acquisition. Biochim. Biophys. Acta-Biomembr. 2020, $1862(9), 183332$.

(19) Ravula, T.; Ramadugu, S. K.; Di Mauro, G. M.; Ramamoorthy, A. Bioinspired, Size-Tunable Self-Assembly of Polymer-Lipid Bilayer Nanodiscs. Angew. Chemie Int. Ed. 2017, 56, $11466-11470$.

(20) Ravula, T.; Ramadugu, S. K.; Di Mauro, G. M.; Ramamoorthy, A. Bioinspired, Size-Tunable Self-Assembly of Polymer-Lipid Bilayer Nanodiscs. Angew. Chemie Int. Ed. 2017, 56, 1146611470.

(21) Sahu, I. D.; Lorigan, G. A. Electron Paramagnetic Resonance as a Tool for Studying Membrane Proteins. Biomolecules 2020, 10 (5).

(22) Mazúr, M.; Valko, M.; Pelikán, P. Quantitative EPR Spectroscopy in Solid State Chemistry. Chem. Pap. 1997, 51 (3), 134-136.

(23) Mazur, M. A Dozen Useful Tips on How to Minimise the Influence of Sources of Error in Quantitative Electron Paramagnetic Resonance (EPR) Spectroscopy - A Review. Anal. Chim. Acta 2006, 561 (1-2), 1-15.

(24) Eaton, G. R.; Eaton, S. S.; Barr, D. P.; Weber, R. T. Quantitative EPR; SpringerWienNewYork, Ed.; New York, 1390.

(25) Dvinskikh, S. V; Zimmermann, H.; Maliniak, A.; Sandström, D. Measurements of Motionally Averaged Heteronuclear Dipolar Couplings in MAS NMR Using R-Type Recoupling. $J$. Magn. Reson. 2004, 168 (2), 194-201.

(26) Ravula, T.; Hardin, N. Z.; Ramadugu, S. K.; Ramamoorthy, A.pH Tunable and Divalent Metal Ion Tolerant Polymer Lipid Nanodiscs. Langmuir 2017, 33 (40), 10655-10662.

(27) Ravula, T.; Hardin, N. Z.; Ramadugu, S. K.; Cox, S. J.; Ramamoorthy, A. Formation of pHResistant Monodispersed Polymer-Lipid Nanodiscs. Angew. Chemie Int. Ed. 2018, 57 (5), 1342-1345. 


\section{Chapter 6}

Future Directions and Conclusions.

Alternative Uses of Copolymer-Based Nanodiscs

The chapter contains some unpublished data, speculations, and conclusions. 


\subsection{Future directions}

\subsubsection{Polymer-nanodiscs in nanomedicine}

Started with the visionary idea that "tiny nanorobots and related machines could be designed, manufactured, and introduced into the human body to perform cellular repairs at the molecular level," the field of nanotechnology has grown exponentially in the last 30 years ${ }^{1}$. Nowadays, it is difficult to think of any industrial sectors not directly affected by nanotechnology. Adhesive/nonadhesive surfaces, biocompatible surfaces in prosthetics, thin-film coatings in the electronic industry, special-paintings, nanosensors, catalysts, and many more nanostructured materials are just a few examples to underline both the variety and importance of nanotechnology for our current and future technology.

Nanomedicine, i.e., nanotechnology applied to medicine, opened exciting frontiers in the diagnostics, pharmaceuticals delivery, and theranostics, offering novel treatments for many human diseases, including cancer and infectious neurological, musculoskeletal, cardiovascular diseases, etc. ${ }^{2}$ (Figure $^{2}$ 6.1). Despite the regulatory challenges, the use of nanomedicine is spreading rapidly, and a growing number of therapeutics have entered routine clinical $\mathrm{use}^{3,4}$.

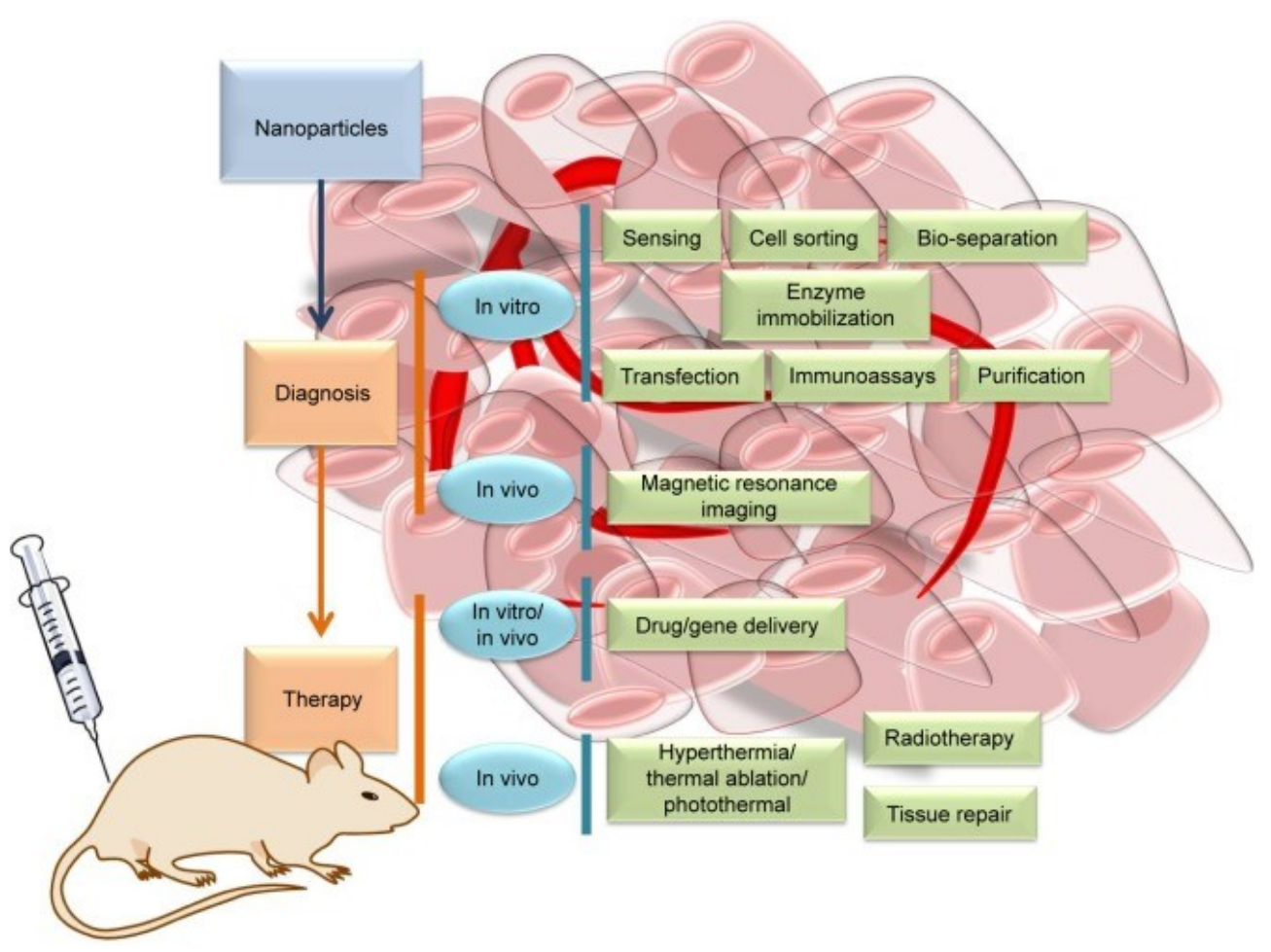

Figure 6.1. Examples of nanomedicine. Different applications of nanoparticles involved in therapy and diagnosis. Figure reproduced with permission from reference ${ }^{5}$. Copyright 2017 Dove Press. 
Pharmaceutical sciences have studied nanoparticles for drug delivery to reduce toxicity and side effects of drugs. The goal is to enhance drug performances while reducing toxicity and eventual side effects. To make drugs more effective, the nanodrugs must accumulate at the target tissue. Thus, the nanoparticles used as carriers must be of sizes compatible with the pores of the target tissues' vasculature. While many substances are currently under investigation, the toxicology should be focused on both the active compound and the nanocarrier. Indeed, the potential interaction with tissues and cells, i.e., the potential toxicity, depends on the actual composition of the nanoparticle formulation ${ }^{4}$.

Many of the systems used to mimic biological membranes and discussed in chapter 1 have been proposed as valuable nanomedicine solutions. For example, the concept of using liposomes as drugcarriers was proposed in the $1970 \mathrm{~s}^{6}$, and since then widely investigated ${ }^{7-14}$. Because of their supramolecular architecture, liposomes can encapsulate both hydrophilic and hydrophobic drugs. Furthermore, they can be functionalized, allowing both active and passive targeting ${ }^{5}$ (Figure 6.2).

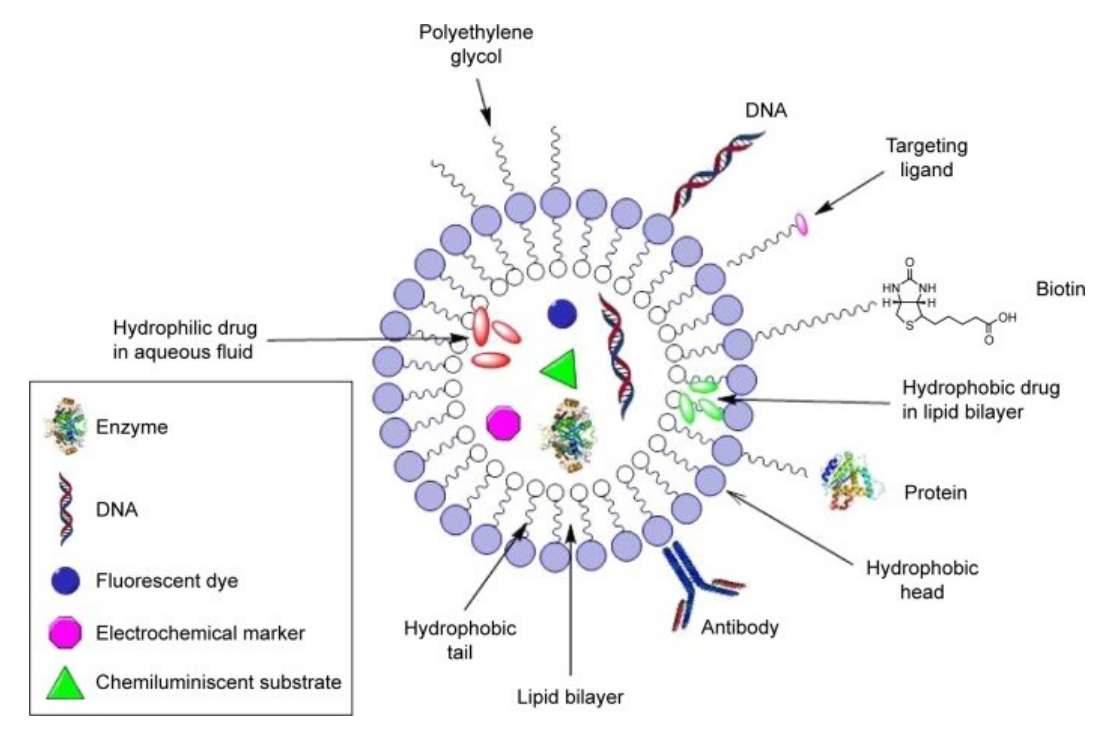

Figure 6.2. Structure of liposomes for drug delivery and diagnostics. Liposomes are colloidal drug carriers consisting of a phospholipid bilayer surface enclosing an aqueous core. Hydrophilic components can be entrapped inside the aqueous core, while the lipophilic components can be incorporated between the lipid bilayers. On the liposomes surface, different particles that target the interest cells can be attached. To avoid the immune system response, the liposomes surface is loaded with a polymer called polyethylene glycol. Thus, the cargo is protected and is discharged into the target cells. Reproduced with permission from reference ${ }^{5}$. Copyright 2017 Dove Press.

Other membrane mimicking systems such as bicelles ${ }^{15-18}$ and MSP-based nanodiscs are considered viable for drug delivery and diagnostics and reported in the literature as well ${ }^{19-21}$. Synthetic macromolecules such as dendrimers ${ }^{22-28}$ and copolymer-micelles ${ }^{29-41}$ are widely investigated for the 
virtually infinite possibilities of functionalization. Figure 6.3 schematically shows examples of polymer-based nanomedicines and their targets.

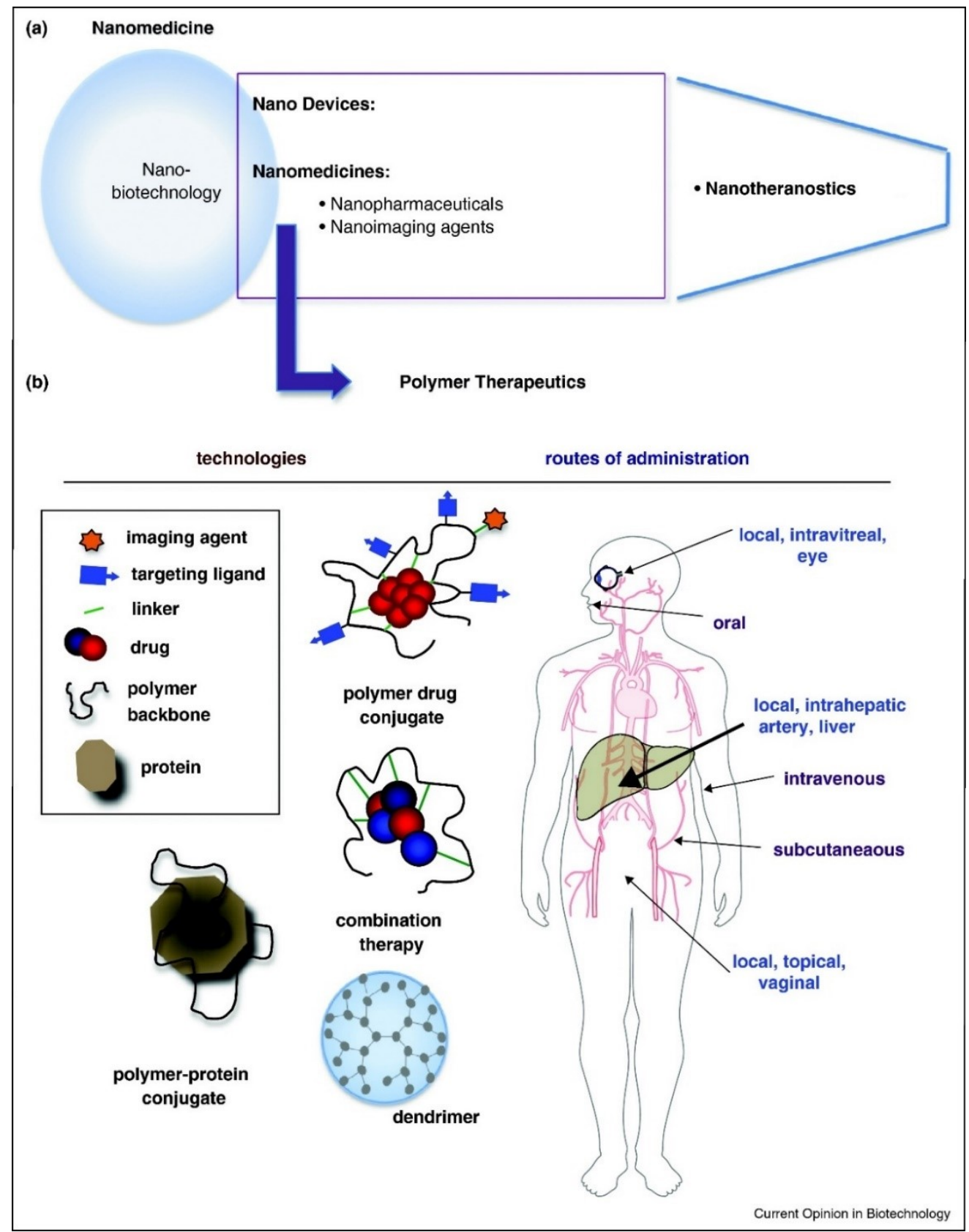

Figure 6.3. Polymer-based nanomedicine. Schematic showing: panel (a) the relationship between nanomedicines and polymer therapeutics, and typical structures of polymer conjugates and their routes of administration. Reproduced with permission from reference ${ }^{3}$. Copyright 2011 Elsevier.

SMA-EA-DOTA copolymer nanodiscs discussed in chapter 3 are hybrid nanoparticles made of phospholipids and synthetic amphipathic copolymers. Initially developed for the investigations of membrane proteins through PRE-NMR and other magnetic resonances techniques ${ }^{42,43}$, they have potential applications in diagnostic as contrast agents for magnetic resonance imaging. 


\subsubsection{Magnetic resonance imaging (MRI) and contrast agents}

Over the years, the development of noninvasive imaging methods offered tremendous opportunities for disease diagnosis and therapeutic intervention ${ }^{44}$. Among these techniques, magnetic resonance imaging is considered a routine diagnostic exam, and every year more than 40 million MRI scans are performed only in the United States ${ }^{45,46}$. MRI and NMR share the same physical principles, and, so far, research has not revealed any risk to health from the static magnetic fields used in the machines of routine exams ${ }^{47}$. Thus, MRI is so popular because (a) is noninvasive, (b) provides threedimensional images with high spatial resolution and high contrast, and (c) does not expose the patient to harmful ionizing radiation.

Magnetic resonance imaging has been possible thanks to the pioneering studies conducted by Paul Lauterbur in the early $1970 \mathrm{~s}^{48,49}$. In recognition of achievements related to the present-day importance of medical research and diagnosis, Lauterbur was awarded the Nobel Prize for Medicine in 2003, shared with the physicist Peter Mansfield.

Lauterbur's experiments used ${ }^{1} \mathrm{H}$ resonances (i) because of their high detection sensitivity and (ii) because all living systems always involve aqueous media. The technique became particularly attractive because relaxation times ( $\mathrm{T}_{1}$ and $\mathrm{T}_{2}$, discussed in Chapter 2 ) of the water protons depend on how water molecules are bound in tissues. Remarkably, one expects to find strong signals from those parts of the body with high-water content, while weak signals are expected from structures containing little water, such as bones. Moreover, water's relaxation properties are also affected by whether the tissues are healthy or diseased, making possible the generation of images with a suitable contrast to reveal solid tumors' exact positions. These images' quality is comparable, if not superior in many cases, to that obtained by X-Ray tomography ${ }^{47}$.

The technique is sensitive to small differences in $T_{1}$ and $T_{2}$. However, it is possible to increase the contrast in MRI images by introducing contrast agents (CA). The first generation of contrast agents, developed between the 1970s and 1980s, included $\mathrm{Gd}^{3+}, \mathrm{Mn}^{2+}$, and $\mathrm{Fe}^{3+}$ complexes and nanoparticles ${ }^{50-53}$. Since then, many other CAs also relying on a variety of mechanisms have been introduced $^{54-56}$. For example, chemical exchange saturation transfer (CEST) and paramagnetic CEST (PARACEST) agents ${ }^{57-60},{ }^{19} \mathrm{~F}$ paramagnetic probes ${ }^{61,62}$, and multimodal agents, i.e., species able to produce MRI contrast and as a probe for other modalities such as positron emission tomography, PET, imaging ${ }^{63}$.

A combination of several factors dictates a good MRI agent: (i) high magnetic moment, (ii) long electron-spin relaxation time, (iii) osmolarity similar to the serum, (iv) low toxicity, (v) high solubility 
in water, (vi) coordinated water molecules, (vii) large molecule with long rotational correlation times ${ }^{47}$. However, each CA is unique, and radiologists must choose among the options knowing both physical and chemical properties. Like any drugs, indeed, contrast agents can cause adverse events and hypersensitivity reactions as well. Thus, radiologists must be aware of the possibility of adverse reactions and balancing them with the clinical benefits of a more accurate diagnosis ${ }^{64}$.

Gadolinium-based contrast agents (GBCA) are the most clinically used CA in MRI because of the electronic properties of $\mathrm{Gd}^{3+}$ ions such as seven unpaired electrons, a high magnetic moment, and long electronic relaxation time ${ }^{55,65,66}$. Free $\mathrm{Gd}^{3+}$, however, is highly toxic ${ }^{67}$. Thus, to be as safe as possible, $\mathrm{Gd}^{3+}$ is administered using thermodynamically stable and kinetically inert chelates ${ }^{55}$. Several formulations approved by the US Food and Drug Administration are available on the market for clinical practice and reported in Table 1. GBCAs are generally classified by the ligands' structure in (i) linear or macrocyclic, (ii) ionic, and non-ionic. Macrocyclic GBCA (e.g., Gadoterate, Gadobutrol, Gadoteridol, etc.) are more stable than the linear (e.g., Gadopentetate, Gadodiamide, etc.). For many years, GBCAs have been considered safe. However, particularly in the last years, linear chelating agents have been under the spotlight. Pieces of evidence of unwanted adverse events ${ }^{68}$ such as allergic reactions ${ }^{69}$, bioaccumulation of $\mathrm{Gd}^{3+}$ ions in bones and brain ${ }^{70-73}$, and nephrogenic systemic fibrosis (NSF) in subjects with impaired renal function ${ }^{74}$ have been piling up. Ionic compounds seem to be more stable than non-ionic and show higher osmolality ${ }^{55,64,67,75}$.

\begin{tabular}{|c|c|c|c|c|c|c|c|c|}
\hline Generic name & Structure & Ionicity & $\mathrm{T}_{1 / 2}$ & $\begin{array}{c}\text { Log } \\
\mathrm{K}_{\text {Therm }}\end{array}$ & $\begin{array}{l}\mathrm{Log} \\
\mathrm{K}_{\text {cond }}\end{array}$ & Stability & NSF risk & $\begin{array}{c}\mathrm{Gd} \\
\text { retention }\end{array}$ \\
\hline Gadodiamate & Linear & Non-ionic & $<5 s$ & 16.9 & 14.9 & Low & High & +++ \\
\hline Gadoversetamide & Linear & Non-ionic & $<5 s$ & 16.6 & 15.0 & Low & High & +++ \\
\hline Gadopentate dimeglumine & Linear & Ionic & $<5 s$ & 22.1 & 17.7 & Intermediate & High & ++ \\
\hline Gadobenate dimeglumine & Linear & Ionic & $<5 s$ & 22.6 & 18.4 & Intermediate & Intermediate & ++ \\
\hline Gadoxetic acid disodium & Linear & Ionic & $<5 s$ & 23.5 & 18.7 & Intermediate & Intermediate & ++ \\
\hline Gadofosveset trisodium & Linear & Ionic & $<5 s$ & 22.1 & 18.9 & Intermediate & Intermediate & ++ \\
\hline Gadoteridol & Macrocyclic & Non-ionic & $3.9 \mathrm{~h}$ & 23.8 & 17.1 & High & Low & + \\
\hline Gadobutrol & Macrocyclic & Non-Ionic & $43 \mathrm{~h}$ & 21.8 & 14.7 & High & Low & + \\
\hline Gadoterate meglumine & Macrocyclic & Non-ionic & $338 \mathrm{~h}$ & 25.6 & 19.3 & High & Low & + \\
\hline
\end{tabular}

Table 6.1. Chemical characteristics of Gadolinium-based contrast agents. Data were taken from reference ${ }^{64}$. 


\subsubsection{Nanodiscs as valid biomimetic MRI contrast agents}

The blood flow through abnormal regions such as tumors is more significant than through the surrounding normal tissues. Besides, the rapid growth of tumors makes blood vessels imperfect, causing the more rapid absorption of any CA. This phenomenon, shown in Figure 6.4, is called enhanced permeability and retention or the EPR effect, and it can be used to design improved $\mathrm{CAs}^{14,76-78}$.

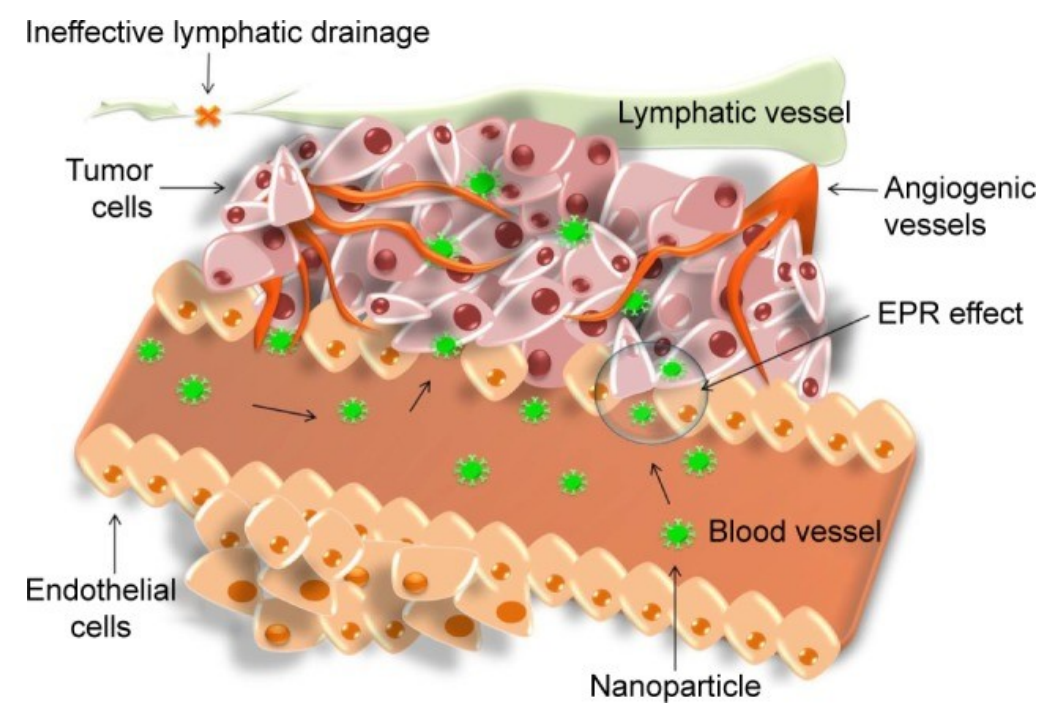

Figure 6.4. Schematics of the EPR effect. Passive targeting relies on cell-specific functions or local environments specific to target the tissue to facilitate uptake and accumulation in tumor tissues and inflammatory sites. Reproduced with permission from reference 5 . Copyright 2017 Dove Press.

Macromolecular MRI CAs composed of multiple $\mathrm{Gd}^{3+}$ chelates proved to be much more efficient and effective in modulating and relaxing water protons than low molecular weight analogs. Furthermore, due to the higher molecular weight, macromolecular CAs are cleared more slowly ${ }^{79-85}$. Copolymernanodiscs are self-assembling colloidal aggregates, describable as nanoparticles made of phospholipid bilayer patches surrounded by an amphipathic belt. These nanoparticles show a supramolecular architecture that imitates the HDL nanoparticles present in the bloodstream. SMAEA-DOTA nanodiscs, discussed in chapter 3 and reproduced in Figure 6.5, might constitute a successful starting point for macromolecular MRI contrast agents. Many are reasons for this speculation. First, as detailed in chapter 3, properties such as size and overall molecular weight make these copolymer-nanodiscs excellent candidates to exploit the EPR effect ${ }^{86-90}$, increasing the loading capacity nanoparticle of the chosen paramagnetic ion. Indeed, SMA-EA-DOTA-based nanodiscs show dimensions in the optimal size range declared for successful drug delivery systems. Second, low toxicity is expected. In fact, their formulation relies on components such as (i) lecithins (i.e., 
DMPC, DPPC, etc.) common components of regular food, (ii) DOTA units, a macrocyclic ionic chelating agent already used in many FDA-approved formulations (Table 1) and not associated with NSF, and (iii) SMA, a copolymer already largely investigated as very low toxic drug carrier ${ }^{91-93}$. The speculation is that the synergy among supramolecular design makes these nanoparticles optimal biocompatible macromolecular contrast agents. In rats, similar systems have shown to be excreted through kidneys ${ }^{21,94}$.

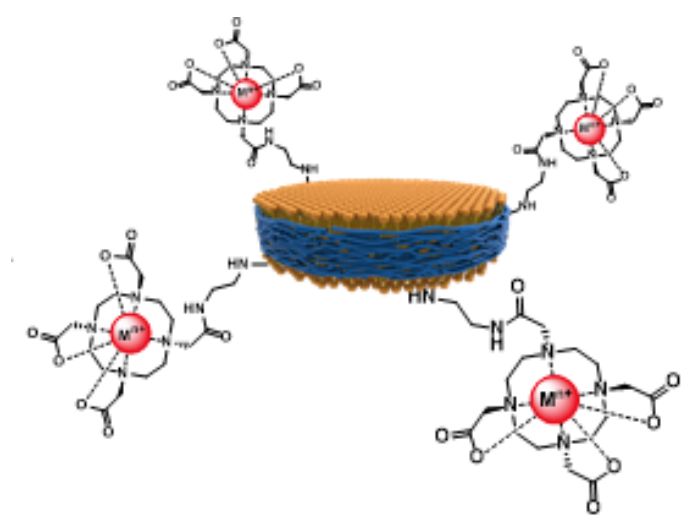

Figure 6.5. SMA-EA-DOTA:lipids nanodiscs for magnetic resonance imaging. The HDL-like supramolecular architecture and the self-assembling nature makes copolymer nanodiscs good MRI CA candidates. All the components are considered to show low toxicity. 


\subsubsection{Preliminary results and discussion}

Compared to the contrast agents reported in Table 6.1, SMA-EA-DOTA-nanodiscs can be classified as ionic (negatively charged) supramolecular contrast agents. Because of multiple copolymer chains per nanodiscs, each nanoparticle has a high- $\mathrm{Gd}^{3+}$ loading capacity compared to the low molecular weight counterparts. Figures 6.6 and 6.7 show preliminary results for inversion recovery experiments on DMPC:SMA-EA-DOTA 1:1 w/w nanodiscs in $\mathrm{D}_{2} \mathrm{O}\left(1 \mathrm{D}{ }^{1} \mathrm{H}\right.$ NMR spectrum is reported in Figure 3.7c, chapter 3). Sample preparation and experimental details are presented in the Materials and Methods section of chapter 3 .

DMPC:SMA-EA 1:1 $(w / w)+G d^{3+}$

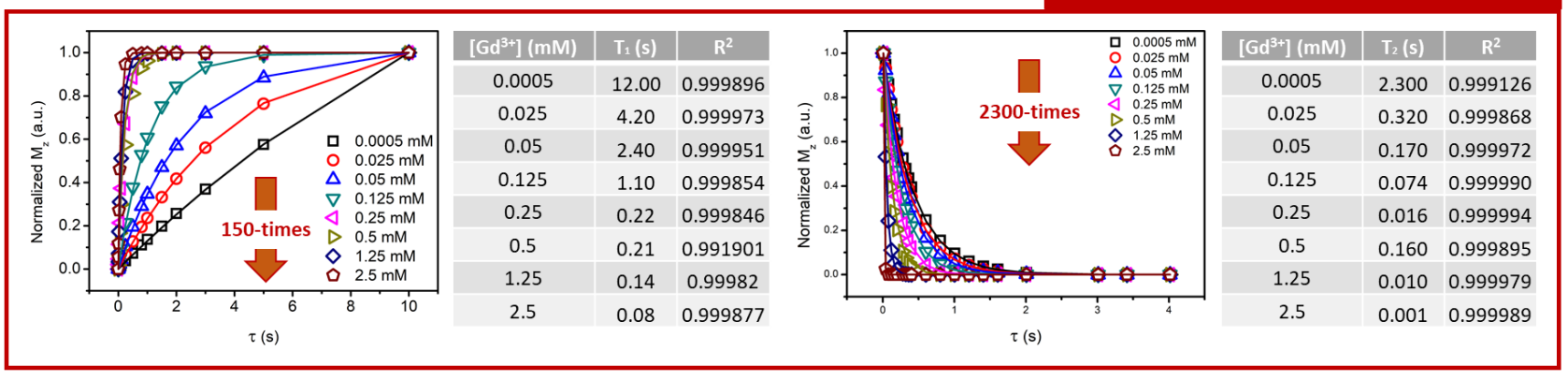

$\left(G d^{3+}\right)$-DMPC:SMA-EA-DOTA 1:1 w/w

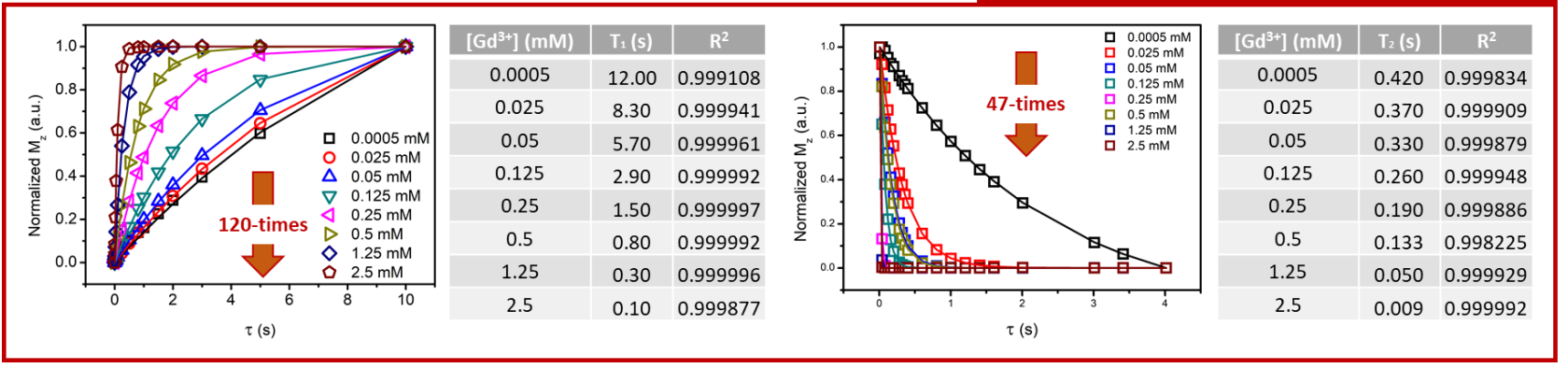

Figure 6.6 - Gd $^{3+}$-loaded DMPC:SMA-EA-DOTA 1:1 w/w nanodises as macromolecular CAs. The figure shows a comparison of the $T_{1}$ and $T_{2}$ times reduction of samples of DMPC:SMA-EA 1:1 (w/w) (top panels) and DMPC:SMAEA-DOTA 1:1 (w/w) (bottom panels) in the presence of $\mathrm{Gd}^{3+}$-ions. As seen in the figure, chelated $\mathrm{Gd}^{3+}$ ions (bottom panels) affect $\mathrm{T}_{1}$ and $\mathrm{T}_{2}$ differently than the free in solution counterpart (top panel).

As expected, both $T_{1}$ and $T_{2}$ of the peak associated with the residual water in the sample $(\sim 4.70 \mathrm{ppm})$ are significantly impacted by increasing $\mathrm{Gd}^{3+}$ ions concentrations. To confirm that this reduction is due to chelated-Gd ${ }^{3+}$ ions, DMPC:SMA-EA-DOTA 1:1 w/w nanodiscs were compared to DMPC:SMA-EA 1:1 w/w nanodiscs with no chelating units. Notably, as reported in Figure 6.6, the free form of $\mathrm{Gd}^{3+}$ ions in solution reduces both $\mathrm{T}_{1}$ and $\mathrm{T}_{2}$ much more than the chelated form; unfortunately, free- $\mathrm{Gd}^{3+}$ ions are toxic; thus, only the chelated form is suitable for MRI applications. 
Copolymer nanodiscs are just the vector containing paramagnetic ions and not the contrast agent per se. To underline the flexibility of this macromolecular contrast agent, also $\mathrm{Mn}^{2+}$-loaded nanodiscs were investigated in the same conditions. Again, the relaxation properties of the sample are drastically affected.

$\left(\mathrm{Mn}^{2+}\right)$-DMPC:SMA-EA-DOTA 1:1 w/w

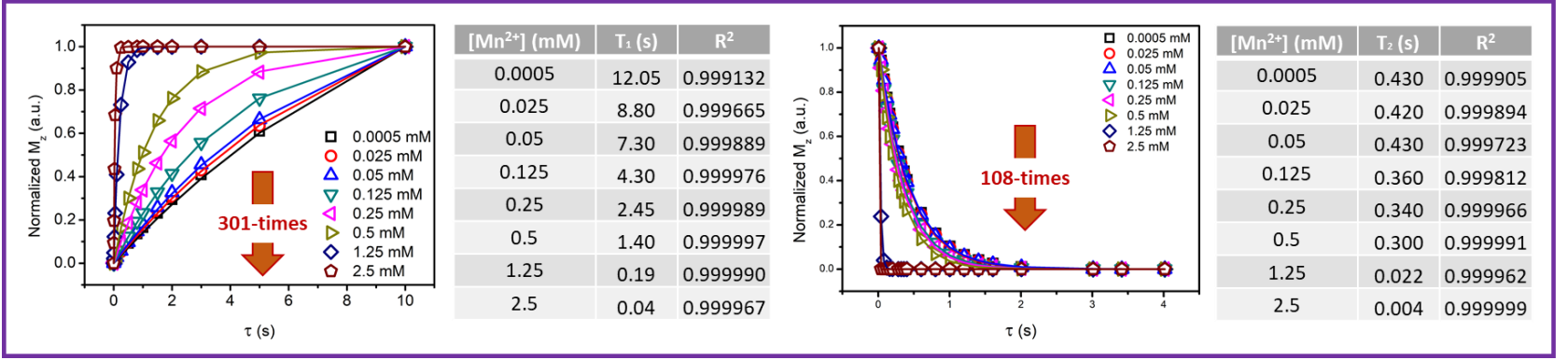

Figure 6.7 - Mn ${ }^{2+}$-loaded DMPC:SMA-EA-DOTA 1:1 w/w nanodiscs as macromolecular CAs. The figure shows a comparison of the $T_{1}$ and $T_{2}$ times reduction of samples of DMPC:SMA-EA-DOTA 1:1 (w/w) loaded with different concentrations of $\mathrm{Mn}^{2+}$ ions.

The main advantage of using $\mathrm{Mn}^{2+}$ in place of $\mathrm{Gd}^{3+}$ would be the absence of NSF risks, but further investigations are needed to investigate both pharmacokinetics and biodistribution of DMPC:SMAEA-DOTA 1:1 (w/w) nanodiscs regardless of the chosen paramagnetic metal. 


\subsection{Conclusions}

Membrane proteins are critical components of any cell, and their malfunction is associated with numerous diseases. For this reason, they represent a primary target for various drugs on the market. Still, academic and pharmaceutical research is hindered by the challenges associated with obtaining stable and functioning samples.

Among all the artificial lipid membranes, copolymer-nanodiscs have shown to be a suitable membrane mimicking system. Moreover, nanodiscs-forming synthetic copolymers can extract membrane proteins directly from their native lipid environment. Acting, de facto, as macromolecular detergents, nanodiscs-forming synthetic copolymers can isolate structurally integer and functionally active membrane proteins into stable discoidal nanoparticles that retain the native lipid composition of the membranes that they were located. Such a feature makes copolymer-nanodiscs a desirable solution for structural biologists, biochemists, and biophysicists.

The Ramamoorthy research group focused on the hydrophilic functionalization of a low molecularweight SMA copolymer. This approach allowed for the tuning and enhancement of these polymers, particularly in the field of NMR spectroscopy.

NMR, widely employed to study nanodiscs reconstituted membrane proteins, suffers from its low intrinsic sensitivity, which necessitates long data acquisition times. PRE is one of the strategies that can be used to enhance the sensitivity of NMR by speeding up the spin-lattice relaxation, a key parameter in assessing the duration of the required data acquisition.

Building up on the hydrophilic functionalization of a low molecular-weight SMA copolymer, this thesis focused on developing novel nanodiscs-forming paramagnetically-labeled copolymers to investigate membrane proteins with magnetic resonance techniques such as PRE-NMR, EPR, and DNP-ssNMR. The goal is to introduce the paramagnetic tags in the sample without significant alteration of the sample. In fact, the current solutions available in the literature involve (i) direct tagging of membrane proteins, (ii) the introduction of modified synthetic lipids, (iii) dissolving paramagnetic species in solution, free to interact in a non-specific way. These approaches, although practical, are invasive and alter the sample.

SMA-EA-DOTA-nanodiscs bridge the advantages of copolymer-nanodiscs as membrane mimetics and PRE effects in NMR, representing a much less invasive approach toward preserving the sample's integrity. As demonstrated in chapter 3, this approach can be used to speed up NMR data acquisition (up to $\sim 50 \%$ ) with minimum-to-no alteration of the spectral quality due to spin-spin relaxation 
enhancement. Comparing the effects of different paramagnetic metals shows that $\mathrm{Gd}^{3+}$ and $\mathrm{Dy}^{3+}$ can be successfully used to shorten $T_{1}$, and so, the recycle delay of NMR experiments. The same idea is explored in chapter 5 to introduce stable organic free radicals such as TEMPO in the copolymer chains of ST-10 and ST-100. As detailed in chapter 5, these copolymers show a substantial reduction of $\mathrm{T}_{1}$-times but are less versatile than the SMA-EA-DOTA. Further investigations are required to assess whether it is more convenient to intervene in the copolymer's final formulation, i.e., by synthesizing new ST-Xs copolymers or whether it is more efficient to work on the final formulation of the copolymer-nanodiscs by merely using mixed copolymers. The belief is that these results can broaden the applications of polymer-nanodiscs in investigating membrane proteins in a native-like environment, using magnetic resonance techniques such as PRE-NMR, EPR, and DNP-ssNMR spectroscopies.

In the comparison of the c.m.c. of a group of commercially available copolymers, pieces of evidence suggest the existence of a chemical equilibrium among the «free» or «micellar» copolymer chains and the «nanodiscs-bound» copolymer chains. Further investigations to evaluate the effect of the removal of free/micellar copolymer on the stability of nanodiscs (unpurified vs. purified behavior) are required to characterize polymers and nanodiscs' properties fully. We speculate that this phenomenon may be exploited to exchange belts in samples of copolymer-lipid nanodiscs. Indeed, a given formulation can be more successful in the extraction but need not be suitable for some biophysical or biochemical techniques. Additionally, a more inexpensive copolymer can be used to extract successfully and stabilize membrane proteins and then be substituted with tagged copolymers, a more expensive solution, optimizing costs and benefits.

Finally, because the introduction of the paramagnetic tags affects $\mathrm{T}_{1}$, it is possible to envision possible uses of these nanoparticles in diagnostics via MRI. As discussed in this chapter 6, factors such as (a) the bioinspired HDL-like shape, (b) the size, and (c) the hybrid composition (copolymer-lipid), make copolymer-nanodiscs suitable in diagnostics and drug delivery. However, in the research for these applications, pharmacokinetics and biodistribution studies are crucial. Thus, further studies are recommended. 


\section{References}

(1) Freitas, R. What Is Nanomedicine? Nanomedicine Nanotechnology, Biol. Med. 2005, 1, 2-9.

(2) Ventola, C. L. The Nanomedicine Revolution: Part 2: Current and Future Clinical Applications. P\&T 2012, 37 (10), 582-591.

(3) Duncan, R. Polymer Therapeutics as Nanomedicines: New Perspectives. Curr. Opin. Biotechnol. 2011, 22 (4), 492-501.

(4) De Jong, W. H.; Borm, P. J. Drug Delivery and Nanoparticles: Applications and Hazards; 2008; Vol. 3.

(5) Jurj, A.; Braicu, C.; Pop, L. A.; Tomuleasa, C.; Gherman, C. D.; Berindan-Neagoe, I. The New Era of Nanotechnology, an Alternative to Change Cancer Treatment. Drug Des. Devel. Ther. 2017, 11, 2871-2890.

(6) Gregoriadis, G. Engineering Liposomes for Drug Delivery: Progress and Problems. Trends Biotechnol. 1995, 13 (12), 527-537.

(7) Litzinger, D. C.; Huang, L. Phosphatodylethanolamine Liposomes: Drug Delivery, Gene Transfer and Immunodiagnostic Applications. BBA - Rev. Biomembr. 1992, 1113 (2), 201227.

(8) Sercombe, L.; Veerati, T.; Moheimani, F.; Wu, S. Y.; Sood, A. K.; Hua, S. Advances and Challenges of Liposome Assisted Drug Delivery. Front. Pharmacol. 2015, 6 (DEC), 1-13.

(9) Schaeffer, H. E.; Krohn, D. L. Liposomes in Topical Drug Delivery. Investig. Ophthalmol. Vis. Sci. 1982, 22 (2), 220-227.

(10) Zhu, Y. X.; Jia, H. R.; Duan, Q. Y.; Liu, X.; Yang, J.; Liu, Y.; Wu, F. G. PhotosensitizerDoped and Plasma Membrane-Responsive Liposomes for Nuclear Drug Delivery and Multidrug Resistance Reversal. ACS Appl. Mater. Interfaces 2020, 12 (33), 36882-36894. 
(11) Lai, W. F.; Wong, W. T.; Rogach, A. L. Molecular Design of Layer-by-Layer Functionalized Liposomes for Oral Drug Delivery. ACS Appl. Mater. Interfaces 2020, 12 (39), 43341-43351.

(12) Chen, Z. J.; Yang, S. C.; Liu, X. L.; Gao, Y.; Dong, X.; Lai, X.; Zhu, M. H.; Feng, H. Y.; Zhu, X. Di; Lu, Q.; et al. Nanobowl-Supported Liposomes Improve Drug Loading and Delivery. Nano Lett. 2020, 20 (6), 4177-4187.

(13) Rayamajhi, S.; Marchitto, J.; Nguyen, T. D. T.; Marasini, R.; Celia, C.; Aryal, S. PHResponsive Cationic Liposome for Endosomal Escape Mediated Drug Delivery. Colloids Surfaces B Biointerfaces 2020, 188, 110804.

(14) Tahara, Y.; Yoshikawa, T.; Sato, H.; Mori, Y.; Zahangir, M. H.; Kishimura, A.; Mori, T.; Katayama, Y. Encapsulation of a Nitric Oxide Donor into a Liposome to Boost the Enhanced Permeation and Retention (EPR) Effect. Medchemcomm 2017, 8 (2), 415-421.

(15) Lin, L.; Wang, X.; Guo, Y.; Ren, K.; Li, X.; Jing, L.; Yue, X.; Zhang, Q.; Dai, Z. Hybrid Bicelles as a pH-Sensitive Nanocarrier for Hydrophobic Drug Delivery. RSC Adv. 2016, 6 (83), 79811-79821.

(16) Rubio, L.; Alonso, C.; Rodríguez, G.; Barbosa-Barros, L.; Coderch, L.; De la Maza, A.; Parra, J. L.; López, O. Bicellar Systems for in Vitro Percutaneous Absorption of Diclofenac. Int. J. Pharm. 2010, 386 (1-2), 108-113.

(17) Lin, L.; Wang, X.; Li, X.; Yang, Y.; Yue, X.; Zhang, Q.; Dai, Z. Modulating Drug Release Rate from Partially Silica-Coated Bicellar Nanodisc by Incorporating PEGylated Phospholipid. Bioconjug. Chem. 2017, 28 (1), 53-63.

(18) Uchida, N.; Horimoto, N. N.; Yamada, K.; Hikima, T.; Ishida, Y. Kinetically Stable Bicelle with Dilution Tolerance, Size Tunability, and Thermoresponsiveness for Drug Delivery Applications. ChemBioChem 2018.

(19) Ng, K. K.; Lovell, J. F.; Vedadi, A.; Hajian, T.; Zheng, G. Self-Assembled Porphyrin Nanodiscs with Structure-Dependent Activation for Phototherapy and Photodiagnostic Applications. ACS Nano 2013, 7 (4), 3484-3490.

(20) Carney, C. E.; Lenov, I. L.; Baker, C. J.; Macrenaris, K. W.; Eckermann, A. L.; Sligar, S. G.; Meade, T. J. Nanodiscs as a Modular Platform for Multimodal MR-Optical Imaging. Bioconjug. Chem. 2015, 26 (5), 899-905.

(21) Huda, P.; Binderup, T.; Pedersen, M. C.; Midtgaard, S. R.; Elema, D. R.; Kjær, A.; Jensen, M.; 
Arleth, L. PET/CT Based in Vivo Evaluation of ${ }^{64} \mathrm{Cu}$ Labelled Nanodiscs in Tumor Bearing Mice. PLoS One 2015, 10 (7).

(22) Liu, M.; Fréchet, J. M. J. Designing Dendrimers for Drug Delivery. Pharm. Sci. Technol. Today 1999, 2 (10), 393-401.

(23) Wijagkanalan, W.; Kawakami, S.; Hashida, M. Designing Dendrimers for Drug Delivery and Imaging: Pharmacokinetic Considerations. Pharm. Res. 2011, 28 (7), 1500-1519.

(24) Caminade, A. M.; Turrin, C. O. Dendrimers for Drug Delivery. J. Mater. Chem. B 2014, 2 (26), 4055-4066.

(25) Wang, H.; Huang, Q.; Chang, H.; Xiao, J.; Cheng, Y. Stimuli-Responsive Dendrimers in Drug Delivery. Biomater. Sci. 2016, 4 (3), 375-390.

(26) Yang, H.; Lopina, S. T.; DiPersio, L. P.; Schmidt, S. P. Stealth Dendrimers for Drug Delivery: Correlation between PEGylation, Cytocompatibility, and Drug Payload. J. Mater. Sci. Mater. Med. 2008, 19 (5), 1991-1997.

(27) Chauhan, A. Dendrimers in Drug Delivery and the Role of "Critical Nanoscale Design Parameters" (CNDPS); Elsevier Inc., 2020.

(28) Huang, D.; Wu, D. Biodegradable Dendrimers for Drug Delivery. Mater. Sci. Eng. C 2018, 90 (October 2017), 713-727.

(29) Kwon, G. S.; Kataoka, K. Block Copolymer Micelles as Long-Circulating Drug Vehicles. Adv. Drug Deliv. Rev. 2012, 64 (SUPPL.), 237-245.

(30) Bachhav, Y. G.; Mondon, K.; Kalia, Y. N.; Gurny, R.; Möller, M. Novel Micelle Formulations to Increase Cutaneous Bioavailability of Azole Antifungals. J. Control. Release 2011, 153 (2), $126-132$.

(31) Kazunori, K.; Glenn S., K.; Masayuki, Y.; Teruo, O.; Yasuhisa, S. Block Copolymer Micelles as Vehicles for Drug Delivery. J. Control. Release 1993, 24 (1-3), 119-132.

(32) Yang, C.; Ebrahim Attia, A. B.; Tan, J. P. K.; Ke, X.; Gao, S.; Hedrick, J. L.; Yang, Y. Y. The Role of Non-Covalent Interactions in Anticancer Drug Loading and Kinetic Stability of Polymeric Micelles. Biomaterials 2012, 33 (10), 2971-2979.

(33) Nasongkla, N.; Bey, E.; Ren, J.; Ai, H.; Khemtong, C.; Guthi, J. S.; Chin, S. F.; Sherry, A. D.; Boothman, D. A.; Gao, J. Multifunctional Polymeric Micelles as Cancer-Targeted, MRI- 
Ultrasensitive Drug Delivery Systems. Nano Lett. 2006, 6 (11), 2427-2430.

(34) Hoppel, M.; Caneri, M.; Glatter, O.; Valenta, C. Self-Assembled Nanostructured Aqueous Dispersions as Dermal Delivery Systems. Int. J. Pharm. 2015, 495 (1), 459-462.

(35) Aliabadi, H. M.; Lavasanifar, A. Polymeric Micelles for Drug Delivery. Expert Opin. Drug Deliv. 2006, 3 (1), 139-162.

(36) Gong, J.; Chen, M.; Zheng, Y.; Wang, S.; Wang, Y. Polymeric Micelles Drug Delivery System in Oncology. J. Control. Release 2012, 159 (3), 312-323.

(37) Yokoyama, M. Clinical Applications of Polymeric Micelle Carrier Systems in Chemotherapy and Image Diagnosis of Solid Tumors. J. Exp. Clin. Med. 2011, 3 (4), 151-158.

(38) Rapoport, N. Physical Stimuli-Responsive Polymeric Micelles for Anti-Cancer Drug Delivery. Prog. Polym. Sci. 2007, 32 (8-9), 962-990.

(39) Kataoka, K.; Harada, A.; Nagasaki, Y. Block Copolymer Micelles for Drug Delivery: Design, Characterization and Biological Significance. Adv. Drug Deliv. Rev. 2012, 64 (SUPPL.), 3748.

(40) Deng, C.; Jiang, Y.; Cheng, R.; Meng, F.; Zhong, Z. Biodegradable Polymeric Micelles for Targeted and Controlled Anticancer Drug Delivery: Promises, Progress and Prospects. Nano Today. 2012, pp 467-480.

(41) Ebrahim Attia, A. B.; Ong, Z. Y.; Hedrick, J. L.; Lee, P. P.; Ee, P. L. R.; Hammond, P. T.; Yang, Y. Y. Mixed Micelles Self-Assembled from Block Copolymers for Drug Delivery. Curr. Opin. Colloid Interface Sci. 2011, 16 (3), 182-194.

(42) Hardin, N. Z.; Kocman, V.; Di Mauro, G. M.; Ravula, T.; Ramamoorthy, A. Metal-Chelated Polymer Nanodiscs for NMR Studies. Angew. Chemie - Int. Ed. 2019, 58 (48), 17246-17250.

(43) Di Mauro, G. M.; Hardin, N. Z.; Ramamoorthy, A. Lipid-Nanodiscs Formed by Paramagnetic Metal Chelated Polymer for Fast NMR Data Acquisition. Biochim. Biophys. Acta-Biomembr. 2020, $1862(9), 183332$.

(44) Weissleder, R.; Pittet, M. J. Imaging in the Era of Molecular Oncology. Nature 2008, 452, $580-589$.

(45) Papanicolas, I.; Woskie, L. R.; Jha, A. Health Care Spending in the United States and Other High-Income Countries. J. Am. Med. Assoc. 2018, 319 (10), 1024-1039. 
(46) Oren, O.; Kebebew, E.; Ioannidis, J. Curbing Unnecessary and Wasted Diagnostic Imaging. J. Am. Med. Assoc. 2019, 321 (3), 245-246.

(47) Cotton, S. Lanthanide and Actinide Chemistry; Wiley, 2006.

(48) Lauterbur, P. C. Image Formation by Induced Local Interactions: Examples Employing Nuclear Magnetic Resonance. Nature 1973, 242, 190-191.

(49) Filler, A. The History, Development and Impact of Computed Imaging in Neurological Diagnosis and Neurosurgery: CT, MRI, and DTI. Nat. Preceedings 2009, 1-76.

(50) Lauffer, R. B. Paramagnetic Metal Complexes as Water Proton Relaxation Agents for NMR Imaging: Theory and Design. Chem. Rev. 1987, 87 (5), 901-927.

(51) Branca, M.; Marciello, M.; Ciuculescu-Pradines, D.; Respaud, M.; Del Puerto Morales, M.; Serra, R.; Casanove, M.-J.; Amiens, C. Towards MRI T2 Contrast Agents of Increased Efficiency. J. Magn. Magn. Mater. 2014, 377, 348-353.

(52) Zhou, Z.; Yang, L.; Gao, J.; Chen, X. Structure-Relaxivity Relationships of Magnetic Nanoparticles for Magnetic Resonance Imaging. Adv. Mater. 2019, 31 (8), 1-32.

(53) Morrow, J. R.; Tóth, É. Next-Generation Magnetic Resonance Imaging Contrast Agents. Inorg. Chem. 2017, 56 (11), 6029-6034.

(54) McMahon, M. T.; Chan, K. W. Y. Developing MR Probes for Molecular Imaging. Adv. Cancer Res. 2014, 124, 297-327.

(55) De Leõn-Rodríguez, L. M.; Martins, A. F.; Pinho, M. C.; Rofsky, N. M.; Sherry, A. D. Basic MR Relaxation Mechanisms and Contrast Agent Design. J. Magn. Reson. Imaging 2015, 42 (3), 545-565.

(56) Bonnet, C. S.; Tóth, É. Smart MR Imaging Agents Relevant to Potential Neurologic Applications. American Journal of Neuroradiology. 2010, pp 401-409.

(57) Sherry, A. D.; Woods, M. Chemical Exchange Saturation Transfer Contrast Agents for Magnetic Resonance Imaging. Annu. Rev. Biomed. Eng. 2008, 10 (1), 391-411.

(58) Vinogradov, E.; Sherry, A. D.; Lenkinski, R. E. CEST: From Basic Principles to Applications, Challenges and Opportunities. J. Magn. Reson. 2013, 229, 155-172.

(59) Woods, M.; Woessner, D. E.; Sherry, A. D. Paramagnetic Lanthanide Complexes as PARACEST Agents for Medical Imaging. Chem. Soc. Rev. 2006, 35 (6), 500-511. 
(60) Hancu, I.; Dixon, W. T.; Woods, M.; Vinogradov, E.; Sherry, A. D.; Lenkinski, R. E. CEST and PARACEST MR Contrast Agents. Acta radiol. 2010, 51 (8), 910-923.

(61) Cakić, N.; Savić, T.; Stricker-Shaver, J.; Truffault, V.; Platas-Iglesias, C.; Mirkes, C.; Pohmann, R.; Scheffler, K.; Angelovski, G. Paramagnetic Lanthanide Chelates for Multicontrast MRI. Chem. Commun. 2016, 52 (59), 9224-9227.

(62) Srivastava, K.; Weitz, E. A.; Peterson, K. L.; Marjańska, M.; Pierre, V. C. Fe- and LnDOTAm-F12 Are Effective Paramagnetic Fluorine Contrast Agents for MRI in Water and Blood. Inorg. Chem. 2017, 56 (3), 1546-1557.

(63) Kumar, A.; Zhang, S.; Hao, G.; Hassan, G.; Ramezani, S.; Sagiyama, K.; Lo, S. T.; Takahashi, M.; Sherry, A. D.; Öz, O. K.; et al. Molecular Platform for Design and Synthesis of Targeted Dual-Modality Imaging Probes. Bioconjug. Chem. 2015, 26 (3), 549-558.

(64) Caschera, L.; Lazzara, A.; Piergallini, L.; Ricci, D.; Tuscano, B.; Vanzulli, A. Contrast Agents in Diagnostic Imaging: Present and Future. Pharmacol. Res. 2016, 110, 65-75.

(65) Caravan, P.; Ellison, J. J.; Mcmurry, T. J.; Lauffer, R. B. Gadolinium(III) Chelates as MRI Contrast Agents: Structure, Dynamics, and Applications. 1999.

(66) Yan, G.-P.; Robinson, L.; Hogg, P. Magnetic Resonance Imaging Contrast Agents: Overview and Perspectives. 2006.

(67) Rogosnitzky, M.; Branch, S. Gadolinium-Based Contrast Agent Toxicity: A Review of Known and Proposed Mechanisms. BioMetals 2016, 29 (3), 365-376.

(68) McDonald, R. J.; McDonald, J. S.; Dai, D.; Schroeder, D.; Jentoft, M. E.; Murray, D. L.; Kadirvel, R.; Eckel, L. J.; Kallmes, D. F. Comparison of Gadolinium Concentrations within Multiple Rat Organs after Intravenous Administration of Linear versus Macrocyclic Gadolinium Chelates. Radiology 2017, 285 (2), 536-545.

(69) Behzadi, A. H.; Zhao, Y.; Farooq, Z.; Prince, M. R. Immediate Allergic Reactions to Gadolinium-Based Contrast Agents: A Systematic Review and Meta-Analysis 1 EVIDENCEBASED PRACTICE: Immediate Allergic Reactions to Gadolinium-Based Contrast Agents Behzadi et Al. Radiology 2018, 286 (2).

(70) Murata, N.; Murata, K.; Gonzalez-Cuyar, L. F.; Maravilla, K. R. Gadolinium Tissue Deposition in Brain and Bone. Magn. Reson. Imaging 2016, 34 (10), 1359-1365.

(71) Radbruch, A. Are Some Agents Less Likely to Deposit Gadolinium in the Brain? 2016. 
(72) Gulani, V.; Calamante, F.; Shellock, F. G.; Kanal, E.; Reeder, S. B. Gadolinium Deposition in the Brain: Summary of Evidence and Recommendations. Lancet Neurol. 2017, 16 (7), 564570.

(73) Fingerhut, S.; Niehoff, A.-C.; Sperling, M.; Jeibmann, A.; Paulus, W.; Niederstadt, T.; Allkemper, T.; Heindel, W.; Holling, M.; Karst, U. Spatially Resolved Quantification of Gadolinium Deposited in the Brain of a Patient Treated with Gadolinium-Based Contrast Agents. J. Trace Elem. Med. Biol. 2018, 45, 125-130.

(74) Kuo, P. H. Gadolinium-Containing MRI Contrast Agents: Important Variations on a Theme for NSF. J. Am. Coll. Radiol. 2008, 5 (1), 29-35.

(75) Fraum, T. J.; Ludwig, D. R.; Bashir, M. R.; Fowler, K. J. Gadolinium-Based Contrast Agents: A Comprehensive Risk Assessment. J. Magn. Reson. Imaging 2017, 46 (2), 338-353.

(76) Kobayashi, H.; Reijnders, K.; English, S.; Yordanov, A. T.; Milenic, D. E.; Sowers, A. L.; Citrin, D.; Krishna, M. C.; Waldmann, T. A.; Mitchell, J. B.; et al. Application of a Macromolecular Contrast Agent for Detection of Alterations of Tumor Vessel Permeability Induced by Radiation. Clin. Cancer Res. 2004, 10 (22), 7712-7720.

(77) Wong, A. D.; Ye, M.; Ulmschneider, M. B.; Searson, P. C. Quantitative Analysis of the Enhanced Permeation and Retention (EPR) Effect. PLoS One 2015, 10 (5), 1-13.

(78) Wong, C.; Stylianopoulos, T.; Cui, J.; Martin, J.; Chauhan, V.; Jiang, W.; Popovic, Z.; Jain, R. K.; Bawendi, M. G.; Fukumura, D. Multistage Nanoparticle Delivery System for Deep Penetration into Tumor Tissue. Proc. Natl. Acad. Sci. U. S. A. 2011, 108 (6), 2426-2431.

(79) Schuhmann-Giampieri, G.; Schmitt-Willich, H.; Frenzel, T.; Press, W.-R.; Weinmann, H.-J. In Vivo and In Vitro Evaluation of Gd-DTPA-Polylysine as a Macromolecular Contrast Agent for Magnetic Resonance Imaging. Invest. Radiol. 1991, 26, 969-974.

(80) Huang, R.; Han, L.; Li, J.; Liu, S.; Shao, K.; Kuang, Y.; Hu, X.; Wang, X.; Lei, H.; Jiang, C. Chlorotoxin-Modified Macromolecular Contrast Agent for MRI Tumor Diagnosis. Biomaterials 2011, 32 (22), 5177-5186.

(81) Kobayashi, H.; Shirakawa, K.; Togashi, K.; Saga, T.; Sato, N.; Hiraga, A.; Konishi, J.; Kawamoto, S.; Watanabe, I.; Heike, Y.; et al. Rapid Accumulation and Internalization of Radiolabeled Herceptin in an Inflammatory Breast Cancer Xenograft with Vasculogenic Mimicry Predicted by the Contrast-Enhanced Dynamic MRI with the Macromolecular Contrast Agent G6-(1B4M-Gd)256. Cancer Res. 2002, 62 (3), 860-866. 
(82) Demsar, F.; Roberts, T. P. L.; Schwickert, H. C.; Shames, D. M.; Van Dijke, C. F.; Mann, J. S.; Saeed, M.; Brasch, R. C. A MRI Spatial Mapping Technique for Microvascular Permeability and Tissue Blood Volume Based on Macromolecular Contrast Agent Distribution. Magn. Reson. Med. 1997, 37 (2), 236-242.

(83) Helbich, T. H.; Gossman, A.; Mareski, P. A.; Radüchel, B.; Roberts, T. P. L.; Shames, D. M.; Mühler, M.; Turetschek, K.; Brasch, R. C. A New Polysaccharide Macromolecular Contrast Agent for MR Imaging: Biodistribution and Imaging Characteristics. J. Magn. Reson. Imaging 2000, $11(6), 694-701$.

(84) Mohs, A. M.; Wang, X.; Goodrich, K. C.; Zong, Y.; Parker, D. L.; Lu, Z. R. PEG-gPoly(GdDTPA-Co-L-Cystine): A Biodegradable Macromolecular Blood Pool Contrast Agent for MR Imaging. Bioconjug. Chem. 2004, 15 (6), 1424-1430.

(85) Kobayashi, H.; Kawamoto, S.; Saga, T.; Sato, N.; Hiraga, A.; Ishimori, T.; Akita, Y.; Mamede, M. H.; Konishi, J.; Togashi, K.; et al. Novel Liver Macromolecular MR Contrast Agent with a Polypropylenimine Diaminobutyl Dendrimer Core: Comparison to the Vascular MR Contrast Agent with the Polyamidoamine Dendrimer Core. Magn. Reson. Med. 2001, 46 (4), 795-802.

(86) Banerjee, A.; Qi, J.; Gogoi, R.; Wong, J.; Mitragotri, S. Role of Nanoparticle Size, Shape and Surface Chemistry in Oral Drug Delivery. J. Control. Release 2016, 238, 176-185.

(87) Gaumet, M.; Vargas, A.; Gurny, R.; Delie, F. Nanoparticles for Drug Delivery: The Need for Precision in Reporting Particle Size Parameters. Eur. J. Pharm. Biopharm. 2008, 69 (1), 1-9.

(88) Singh, R.; Lillard, J. W. Nanoparticle-Based Targeted Drug Delivery. Exp. Mol. Pathol. 2009, $86(3), 215-223$.

(89) Hans, M. .; Lowman, A. . Biodegradable Nanoparticles for Drug Delivery and Targeting. Curr. Opin. Solid State Mater. Sci. 2002, 6 (4), 319-327.

(90) Dreaden, E. C.; Austin, L. A.; MacKey, M. A.; El-Sayed, M. A. Size Matters: Gold Nanoparticles in Targeted Cancer Drug Delivery. Ther. Deliv. 2012, 3 (4), 457-478.

(91) Maeda, H.; Ueda, M.; Morinaga, T.; Matsumotog, T. Conjugation of Poly(Styrene-Co -Maleic Acid) Derivatives to the Antitumor Protein Neocarzinostatin: Pronounced Improvements in Pharmacological Properties. J. Med. Chem 1985, 28, 455-461.

(92) Kobayashi, A.; Oda, T.; Maeda, H. Protein Binding of Macromolecular Anticancer Agent SMANCS: Characterization of Poly(Styrene-Co-Maleic Acid) Derivatives as an Albumin 
Binding Ligand. J. Bioact. Compat. Polym. 1988, 3 (4), 319-333.

(93) Larson, N.; Greish, K.; Bauer, H.; Maeda, H.; Ghandehari, H. Synthesis and Evaluation of Poly(Styrene-Co-Maleic Acid) Micellar Nanocarriers for the Delivery of Tanespimycin. Int. J. Pharm. 2011, 420 (1), 111-117.

(94) Zhou, Z.; Wang, L.; Chi, X.; Bao, J.; Yang, L.; Zhao, W.; Chen, Z.; Wang, X.; Chen, X.; Gao, J. Engineered Iron-Oxide-Based Nanoparticles as Enhanced $\mathrm{T}_{1}$ Contrast Agents for Efficient Tumor Imaging. ACS Nano 2013, 7 (4), 3287-3296. 


\section{Appendix}

\section{Supporting Information for Chapter 3}

\section{"Lipid-Nanodiscs Formed by Paramagnetic Metal Chelated Polymer for Fast NMR Data Acquisition"}

The content for this appendix includes data from the supporting information for the following reference:

Di Mauro Giacomo M., Hardin Nathaniel Z., Ramamoorthy Ayyalusamy, Lipid-nanodiscs formed by paramagnetic metal chelated polymer for fast NMR data acquisition, BBA Biomembranes, $1862,9,09 / 2020,183332$

DOI: 10.1016/j.bbamem.2020.183332 
a) DMPC:SMA-EA 1:1 (w/w) vs [Gd $\left.{ }^{3+}\right]$

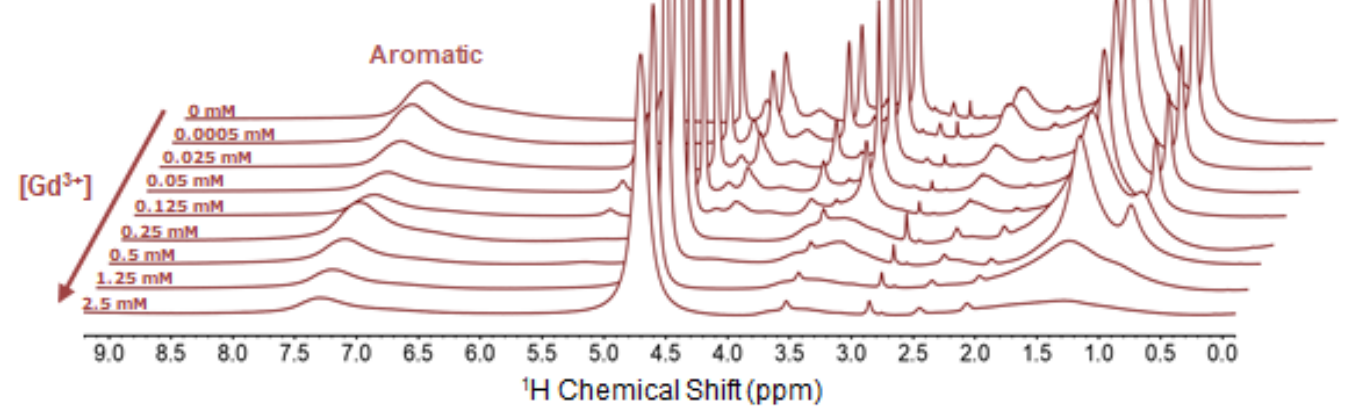

b)

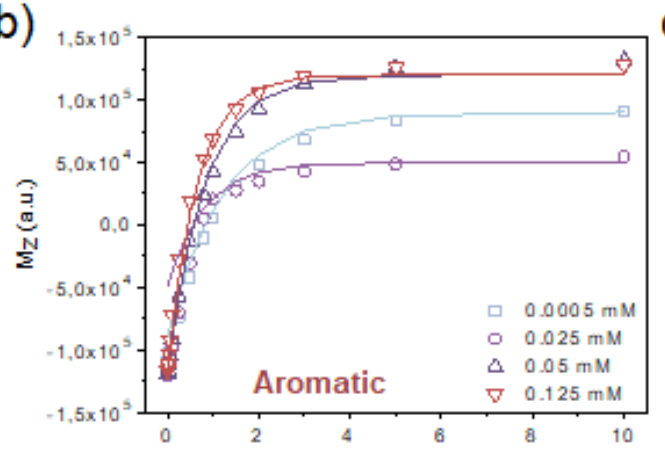

d)

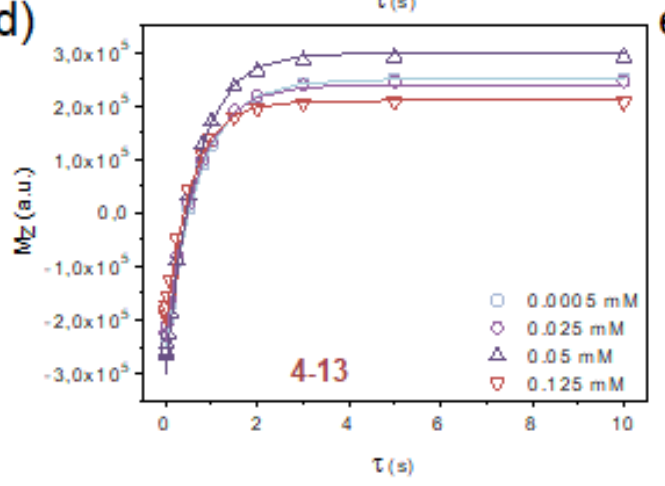

c)

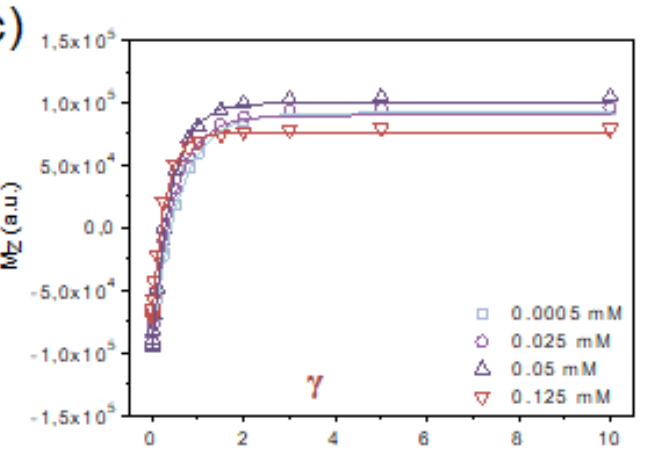

e)

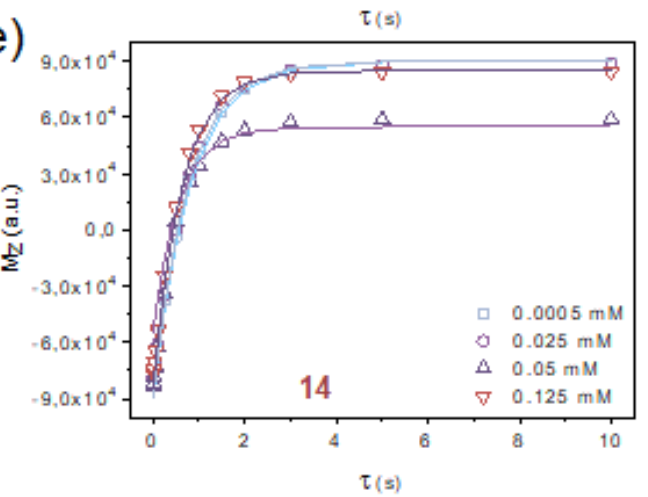

Figure S1. T1 measurements for DMPC: SMA-EA 1:1 (w/w) macro-nanodiscs. a) the stacking of 1D ${ }^{1} \mathrm{H}-\mathrm{NMR}$ spectra in the presence of different concentrations of $\left[\mathrm{Gd}^{3+}\right]$ ions. b), c), d), and e) inversion recovery fitting plots of data for the selected ${ }^{1} \mathrm{H}$ peaks at various $\left[\mathrm{Gd}^{3+}\right]$ concentrations.

\begin{tabular}{|c|cccc|}
\cline { 2 - 5 } \multicolumn{1}{c|}{} & \multicolumn{4}{|c|}{${ }^{1}$ H Peaks } \\
\hline$\left[\mathbf{G d}^{3+}\right] \mathbf{( m M )}$ & Aromatic $(\mathbf{s})$ & $\boldsymbol{\gamma}(\mathbf{s})$ & $\mathbf{4 - 1 3}(\mathbf{s})$ & $\mathbf{1 4}(\mathbf{s})$ \\
\hline 0 & 1.68 & 0.58 & 0.74 & 0.80 \\
0.0005 & 1.20 & 0.58 & 0.72 & 0.81 \\
0.025 & 0.80 & 0.48 & 0.67 & 0.76 \\
0.05 & 0.82 & 0.40 & 0.65 & 0.56 \\
0.125 & 0.64 & 0.27 & 0.56 & 0.62 \\
0.25 & $\mathrm{~N} / \mathrm{D}$ & $\mathrm{N} / \mathrm{D}$ & $\mathrm{N} / \mathrm{D}$ & $\mathrm{N} / \mathrm{D}$ \\
0.5 & $\mathrm{~N} / \mathrm{D}$ & $\mathrm{N} / \mathrm{D}$ & $\mathrm{N} / \mathrm{D}$ \\
1.25 & $\mathrm{~N} / \mathrm{D}$ & $\mathrm{N} / \mathrm{D}$ & $\mathrm{N} / \mathrm{D}$ \\
2.5 & $\mathrm{~N} / \mathrm{D}$ & $\mathrm{N} / \mathrm{D}$ & $\mathrm{N} / \mathrm{D}$ \\
\hline
\end{tabular}

Table S1. T 1 values measured from DMPC:SMA-EA 1:1 (w/w) macro-nanodiscs for varying $\left[\mathbf{G d}^{3+}\right]$. Values marked as N/D are data not determined due to significant line-broadening due to the presence of paramagnetic ions. 


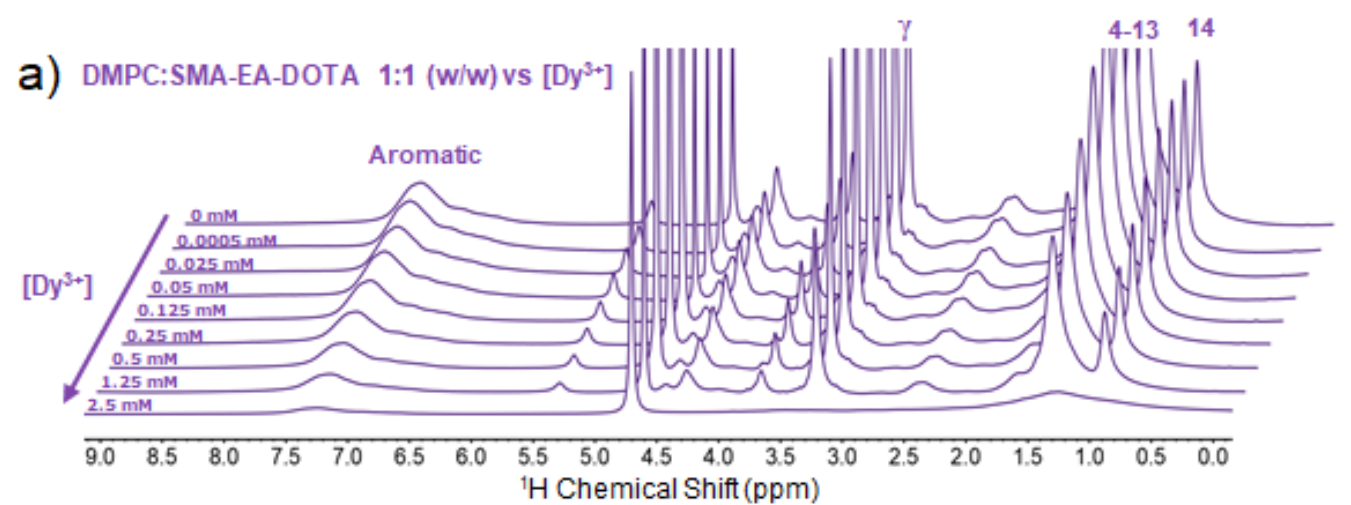

b)

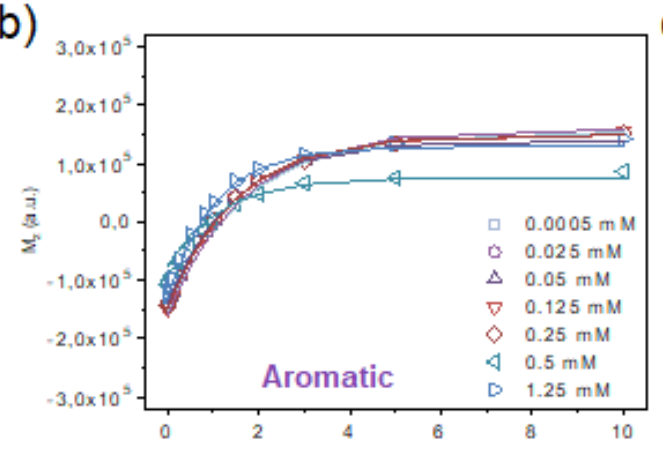

d)

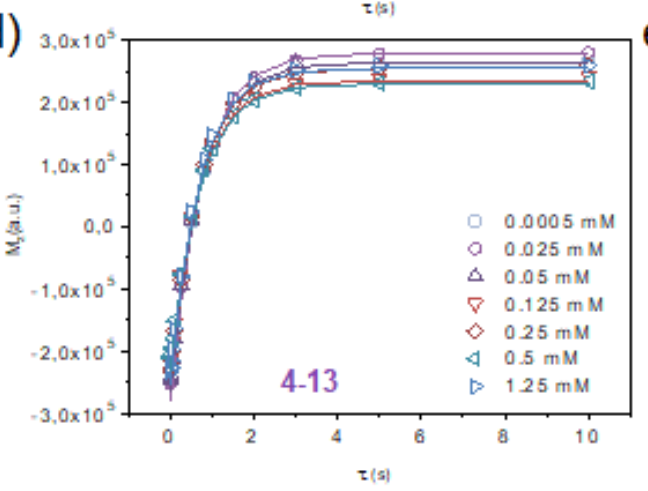

c)

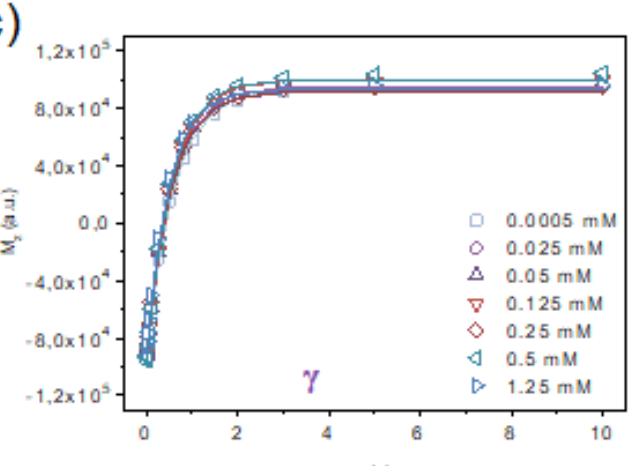

e)

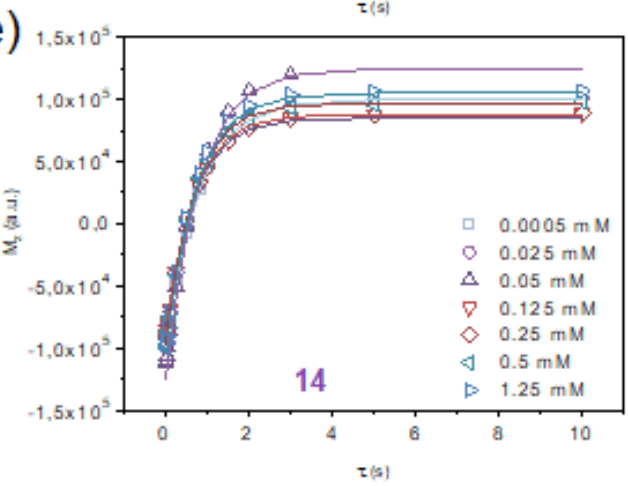

Figure S2. T1 measurements for DMPC: SMA-EA-DOTA 1:1 (w/w) macro-nanodises. a) the stacking of $1 \mathrm{D}^{1} \mathrm{H}-$ NMR spectra in the presence of different concentrations of $\left[D y^{3+}\right]$ ions. b), c), d), and e) inversion recovery plots for the selected ${ }^{1} \mathrm{H}$ peaks at various $\left[\mathrm{Dy}^{3+}\right]$ concentrations

\begin{tabular}{|c|cccc|}
\cline { 2 - 5 } \multicolumn{1}{c|}{} & \multicolumn{4}{c|}{${ }^{1}$ H Peaks } \\
\hline$\left[\mathbf{D y}^{\mathbf{3}+}\right] \mathbf{( m M )}$ & Aromatic (s) & $\boldsymbol{\gamma}(\mathbf{s})$ & $\mathbf{4 - 1 3}(\mathbf{s})$ & $\mathbf{1 4}(\mathbf{s})$ \\
\hline 0 & 1.65 & 0.59 & 0.70 & 0.80 \\
0.0005 & 1.69 & 0.60 & 0.74 & 0.80 \\
0.025 & 1.67 & 0.55 & 0.74 & 0.80 \\
0.05 & 1.44 & 0.55 & 0.73 & 0.80 \\
0.125 & 1.51 & 0.55 & 0.70 & 0.69 \\
0.25 & 1.48 & 0.54 & 0.70 & 0.68 \\
0.5 & 1.18 & 0.52 & 0.70 & 0.70 \\
1.25 & 1.05 & 0.48 & 0.68 & 0.72 \\
2.5 & N/D & N/D & N/D & N/D \\
\hline
\end{tabular}

Table S2. $T_{1}$ values of DMPC: SMA-EA-DOTA 1:1 (w/w) macro nanodises for varying $\left[\mathrm{Dy}^{3+}\right]$. Values marked as N/D are data not determined due to significant line-broadening due to the presence of paramagnetic ions. 


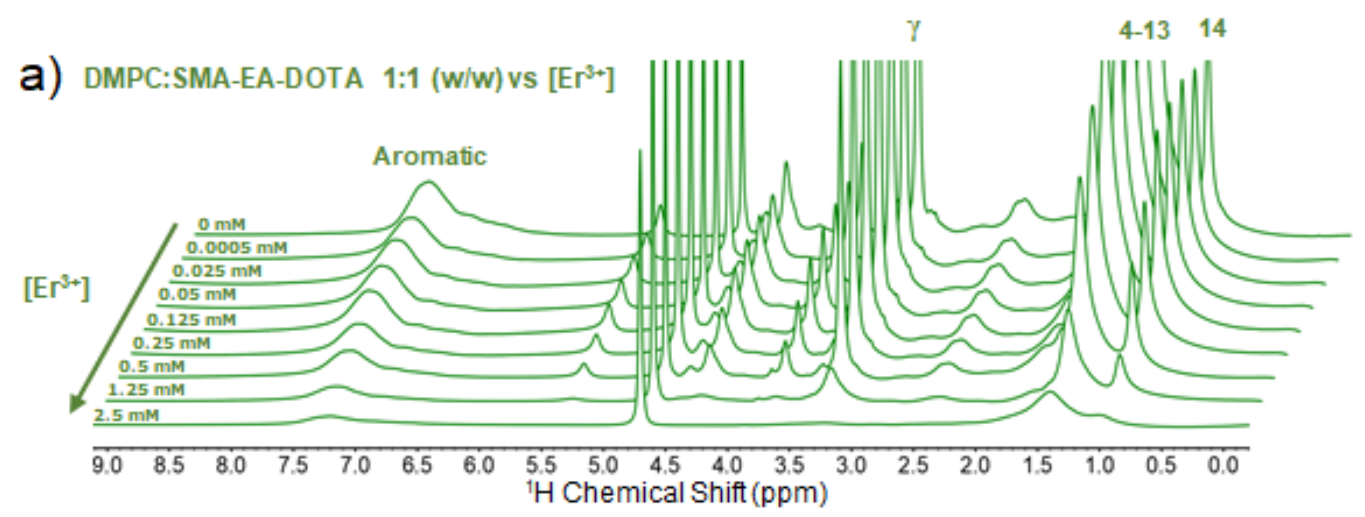

b)

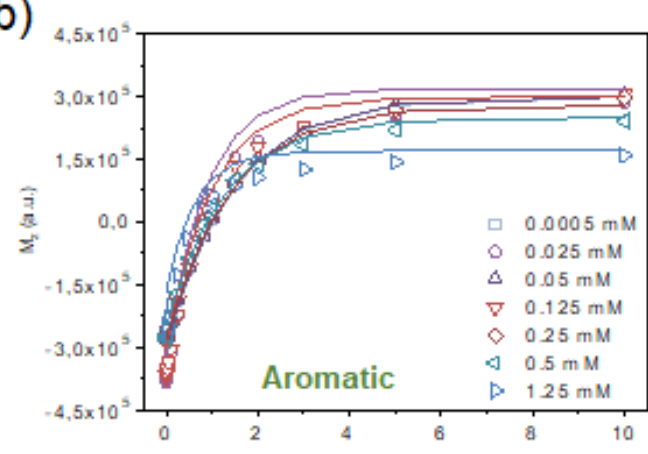

d)

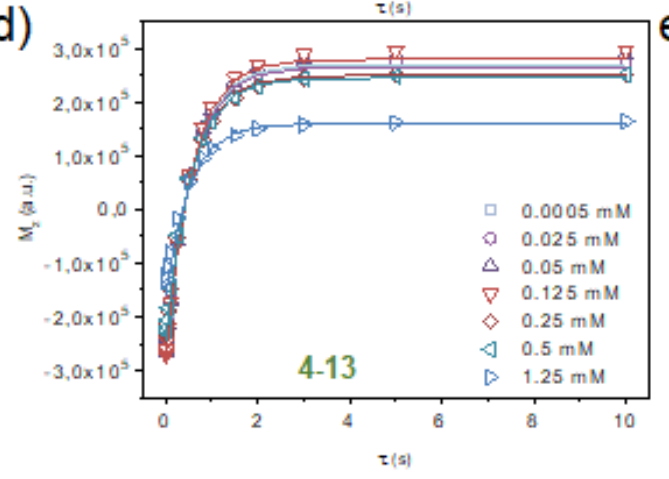

c)

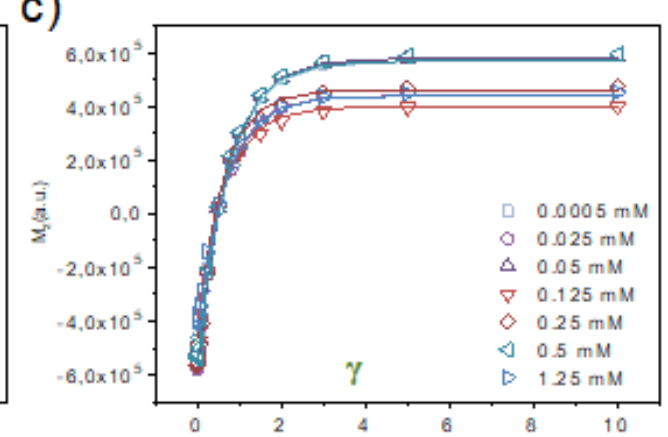

e)

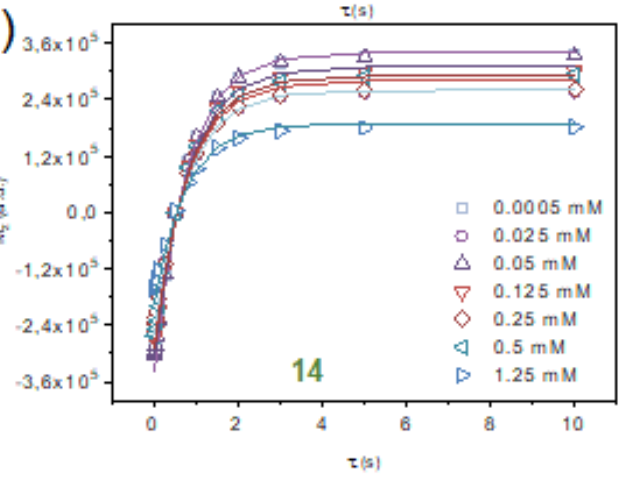

Figure S3. T$_{1}$ measurements for DMPC: SMA-EA-DOTA 1:1 (w/w) macro-nanodises. a) the stacking of $1 \mathrm{D}{ }^{1} \mathrm{H}-$ NMR spectra in the presence of different concentrations of $\left[\mathrm{Er}^{3+}\right]$ ions. b), c), d), and e) inversion recovery plots for the selected ${ }^{1} \mathrm{H}$ peaks at various $\left[\mathrm{Er}^{3+}\right]$ concentrations.

\begin{tabular}{|c|cccc|}
\cline { 2 - 5 } \multicolumn{1}{c|}{} & \multicolumn{4}{|c|}{${ }^{\mathbf{1}}$ H Peaks } \\
\hline$\left[\mathbf{E r}^{\mathbf{3}}\right] \mathbf{( m M )}$ & Aromatic $(\mathbf{s})$ & $\boldsymbol{\gamma}(\mathbf{s})$ & $\mathbf{4 - 1 3}(\mathbf{s})$ & $\mathbf{1 4}(\mathbf{s})$ \\
\hline 0 & 1.65 & 0.59 & 0.70 & 0.80 \\
0.0005 & 1.48 & 0.56 & 0.73 & 0.77 \\
0.025 & 0.88 & 0.56 & 0.66 & 0.78 \\
0.05 & 1.46 & 0.56 & 0.72 & 0.77 \\
0.125 & 1.00 & 0.56 & 0.66 & 0.78 \\
0.25 & 1.42 & 0.56 & 0.60 & 0.78 \\
0.5 & 1.10 & 0.55 & 0.70 & 0.77 \\
1.25 & 0.62 & 0.52 & 0.68 & 0.75 \\
2.5 & $\mathrm{~N} / \mathrm{D}$ & $\mathrm{N} / \mathrm{D}$ & $\mathrm{N} / \mathrm{D}$ & $\mathrm{N} / \mathrm{D}$ \\
\hline
\end{tabular}

Table S3. $T_{1}$ values of DMPC: SMA-EA-DOTA 1:1 (w/w) macro nanodiscs for varying $\left[\mathbf{E r}^{3+}\right]$. Values marked as N/D are data not determined due to significant line-broadening due to the presence of paramagnetic ions. 


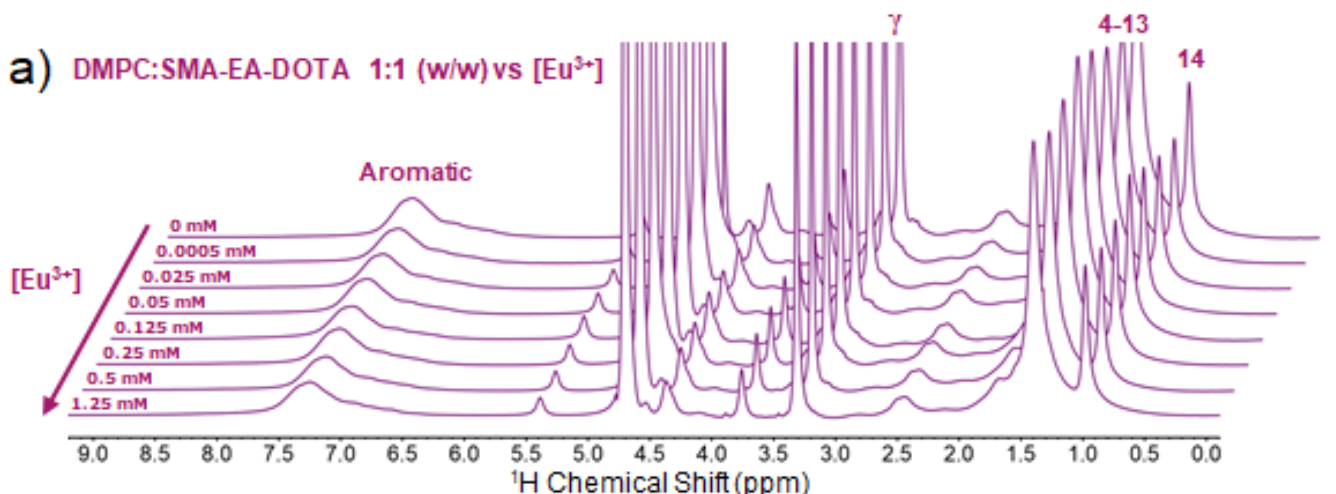

b)

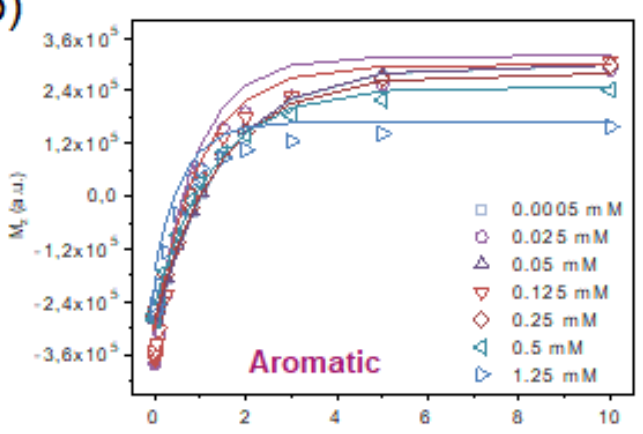

d)

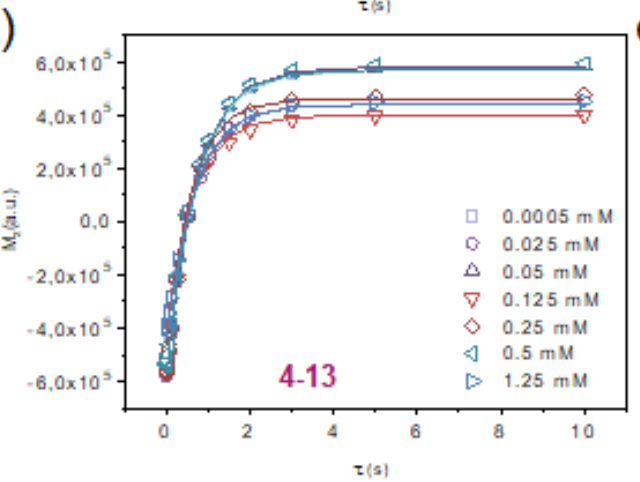

c)

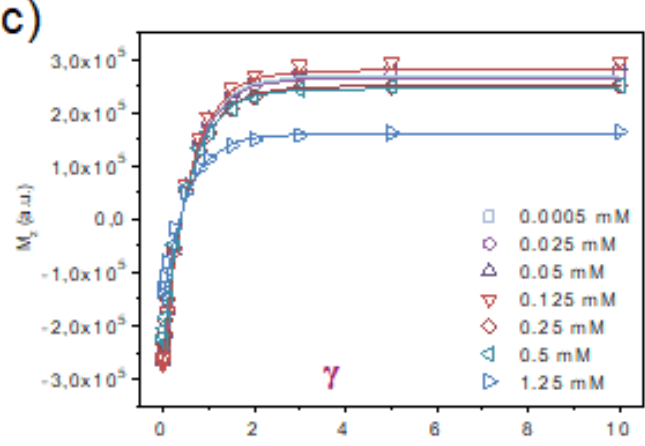

e)

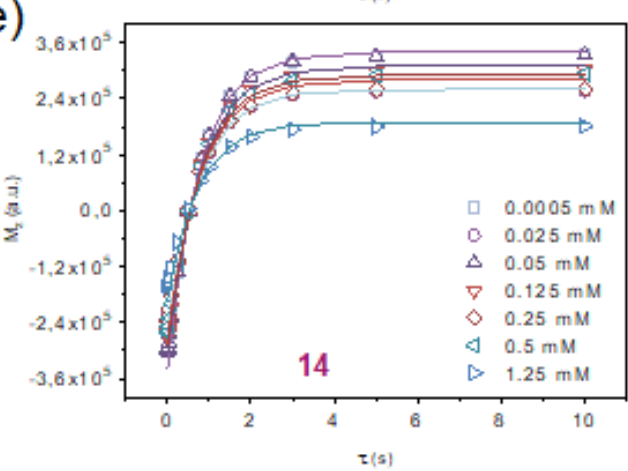

Figure S4. T1 measurements for DMPC: SMA-EA-DOTA 1:1 (w/w) macro-nanodiscs. a) the stacking of $1 \mathrm{D}{ }^{1} \mathrm{H}-$ NMR spectra in the presence of different concentrations of $\left[\mathrm{Eu}^{3+}\right]$ ions. b), c), d), and e) inversion recovery fitting plots for the selected ${ }^{1} \mathrm{H}$ peaks at various $\left[\mathrm{Eu}^{3+}\right]$ concentrations.

\begin{tabular}{|c|cccc|}
\cline { 2 - 5 } \multicolumn{1}{c|}{} & \multicolumn{4}{c|}{${ }^{\mathbf{1}}$ H Peaks } \\
\hline$\left[\mathbf{E u}^{\mathbf{3}+}\right] \mathbf{( m M )}$ & Aromatic $(\mathbf{s})$ & $\boldsymbol{\gamma}(\mathbf{s})$ & $\mathbf{4 - 1 3}(\mathbf{s})$ & $\mathbf{1 4}(\mathbf{s})$ \\
\hline 0 & 1.65 & 0.59 & 0.70 & 0.80 \\
0.0005 & 1.85 & 0.66 & 0.72 & 0.78 \\
0.025 & 1.90 & 0.60 & 0.72 & 0.76 \\
0.05 & 1.76 & 0.58 & 0.74 & 0.80 \\
0.125 & 1.68 & 0.58 & 0.74 & 0.80 \\
0.25 & 1.70 & 0.60 & 0.74 & 0.80 \\
0.5 & 1.70 & 0.58 & 0.73 & 0.80 \\
1.25 & 1.64 & 0.60 & 0.74 & 0.80 \\
2.5 & N/D & N/D & N/D & N/D \\
\hline
\end{tabular}

Table S4. T 1 values of DMPC:SMA-EA-DOTA 1:1 (w/w) macro nanodises for varying $\left[\mathrm{Eu}^{3+}\right]$. Values marked as N/D are data not determined due to significant line-broadening due to the presence of paramagnetic ions. 


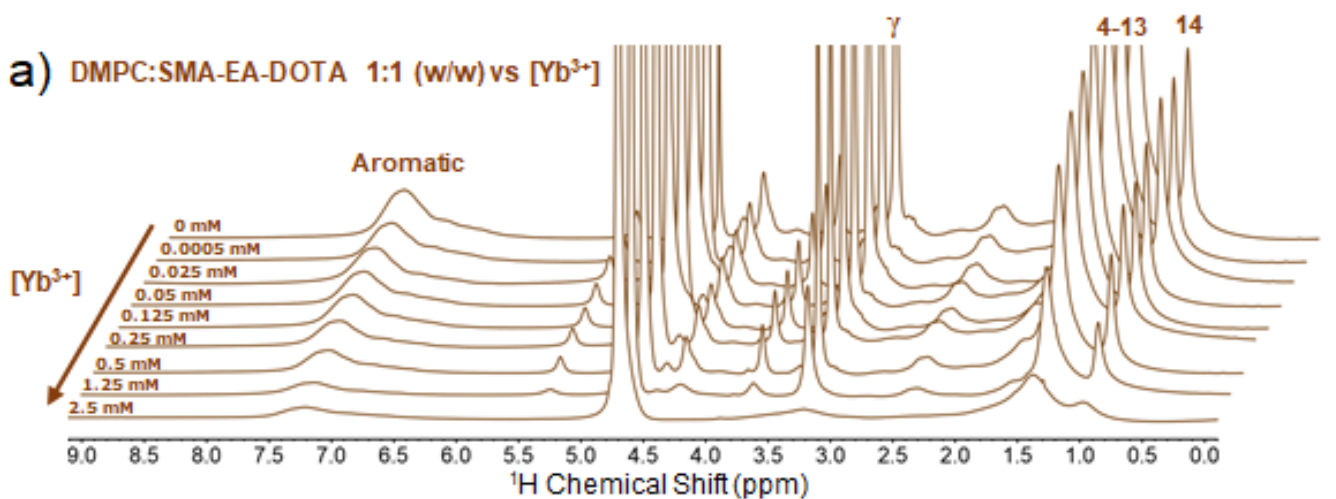

b)

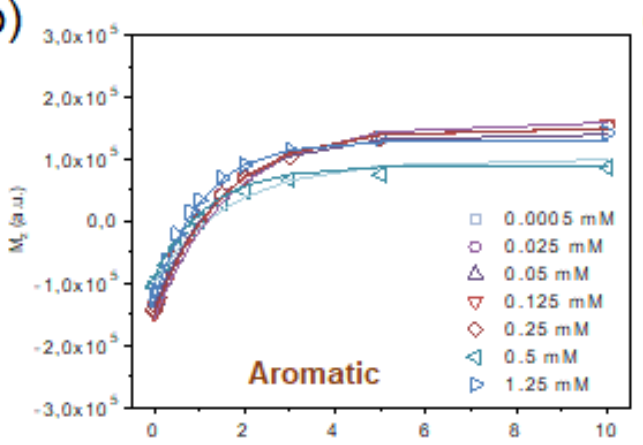

d)

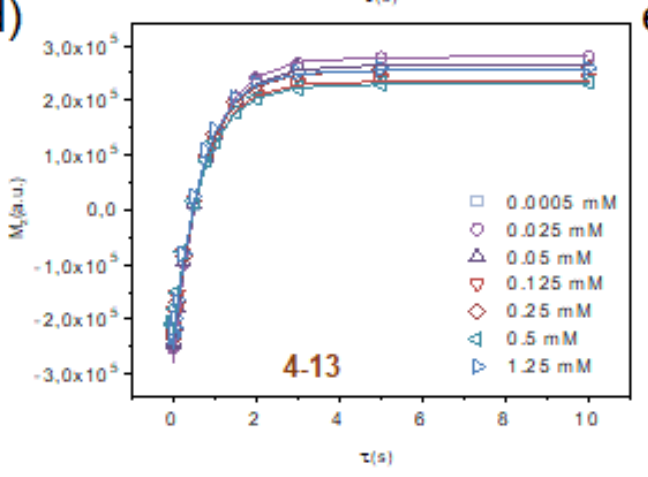

c)

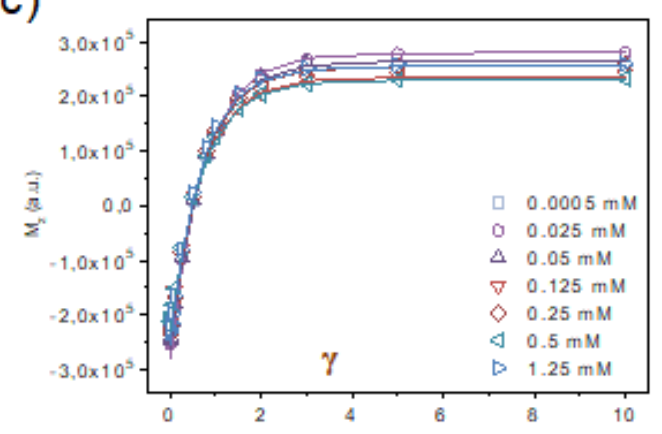

e)

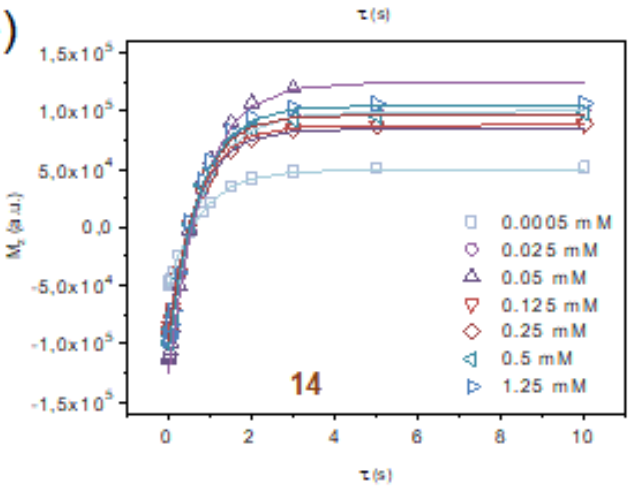

Figure S5. T1 measurements for DMPC: SMA-EA-DOTA 1:1 (w/w) macro-nanodiscs. a) the stacking of $1 \mathrm{D}{ }^{1} \mathrm{H}-$ NMR spectra in the presence of different concentrations of $\left[\mathrm{Yb}^{3+}\right]$ ions. b), c), d), and e) inversion recovery plots for the selected ${ }^{1} \mathrm{H}$ peaks at various $\left[\mathrm{Yb}^{3+}\right]$ concentrations.

\begin{tabular}{|c|cccc|}
\cline { 2 - 5 } \multicolumn{1}{c|}{} & \multicolumn{4}{c|}{${ }^{1}$ H Peaks } \\
\hline$\left.\left[\mathbf{Y b}^{\mathbf{3}+}\right] \mathbf{( m M}\right)$ & Aromatic (s) & $\boldsymbol{\gamma}(\mathbf{s})$ & $\mathbf{4 - 1 3}(\mathbf{s})$ & $\mathbf{1 4}(\mathbf{s})$ \\
\hline 0 & 1.65 & 0.59 & 0.70 & 0.80 \\
0.0005 & 1.78 & 0.58 & 0.73 & 0.78 \\
0.025 & 1.78 & 0.58 & 0.73 & 0.78 \\
0.05 & 1.74 & 0.58 & 0.73 & 0.80 \\
0.125 & 1.72 & 0.58 & 0.72 & 0.80 \\
0.25 & 1.72 & 0.57 & 0.73 & 0.78 \\
0.5 & 1.67 & 0.57 & 0.72 & 0.79 \\
1.25 & 1.66 & 0.54 & 0.71 & 0.77 \\
2.5 & N/D & N/D & N/D & N/D \\
\hline
\end{tabular}

Table S5. $\mathbf{T}_{1}$ values of DMPC:SMA-EA-DOTA 1:1 $(\mathrm{w} / \mathrm{w})$ macro nanodises for varying $\left[\mathrm{Yb}^{3+}\right]$. Values marked as N/D are data not determined due to significant line-broadening due to the presence of paramagnetic ions. 
a) DMPC:SMA-EA-DOTA $1: 1(\mathrm{w} / \mathrm{w})$ vs $\left[\mathrm{Eu}^{3+}\right]$

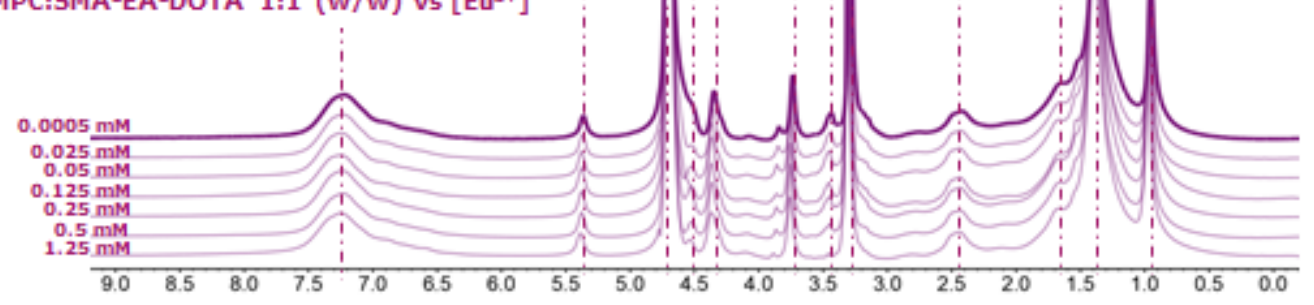

b) DMPC:SMA-EA-DOTA $1: 1(\mathrm{w} / \mathrm{w})$ vs $\left[\mathrm{Gd}^{3+}\right]$

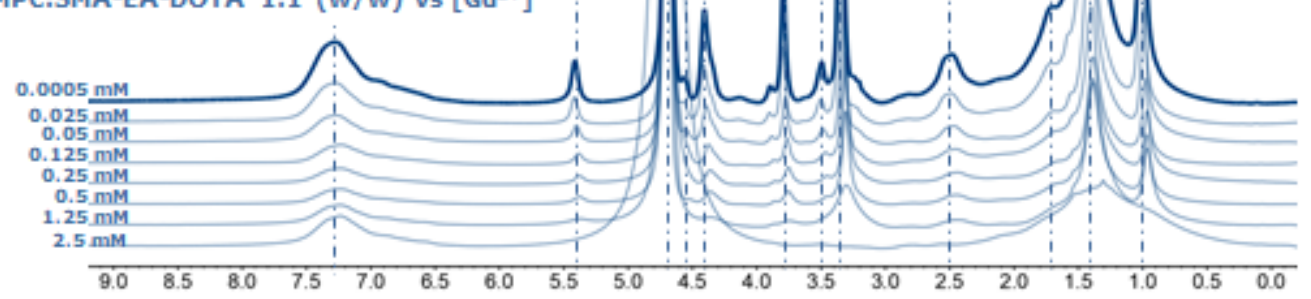

C) DMPC:SMA-EA $1: 1(\mathrm{w} / \mathrm{w})$ vs $\left[\mathrm{Dy}^{3+}\right]$

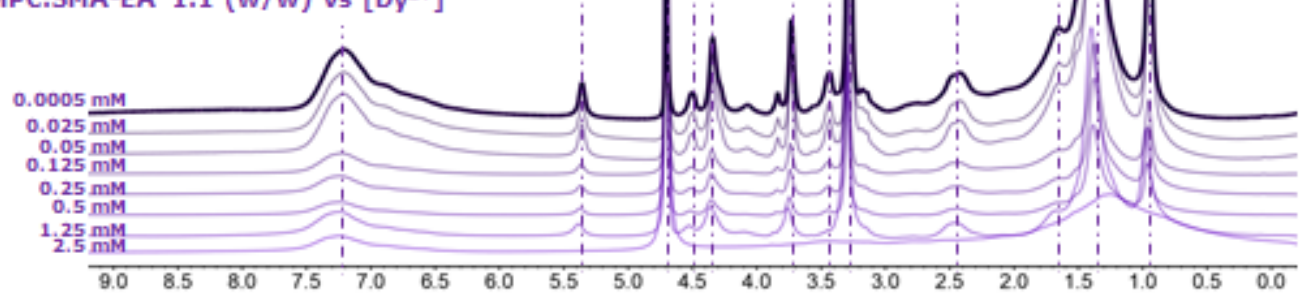

d) DMPC:SMA-EA-DOTA $1: 1(\mathrm{w} / \mathrm{w})$ vs $\left[\mathrm{Er}^{3+}\right]$

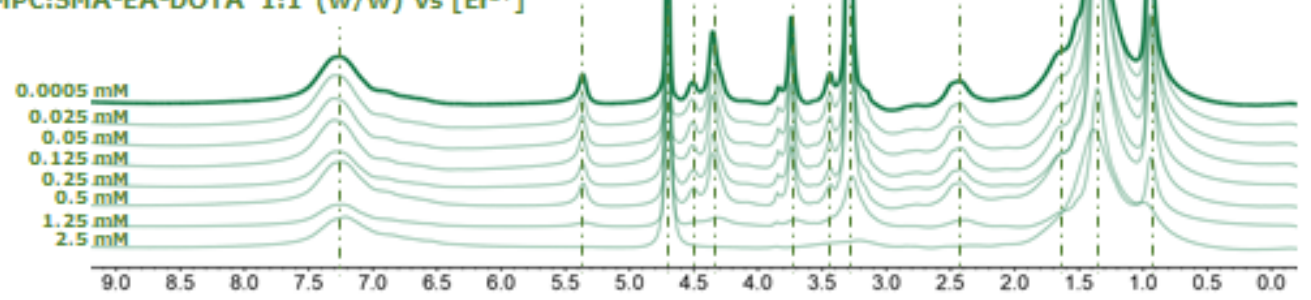

e) DMPC:SMA-EA-DOTA $1: 1(\mathrm{w} / \mathrm{w})$ vs $\left[\mathrm{Yb}^{3+}\right]$

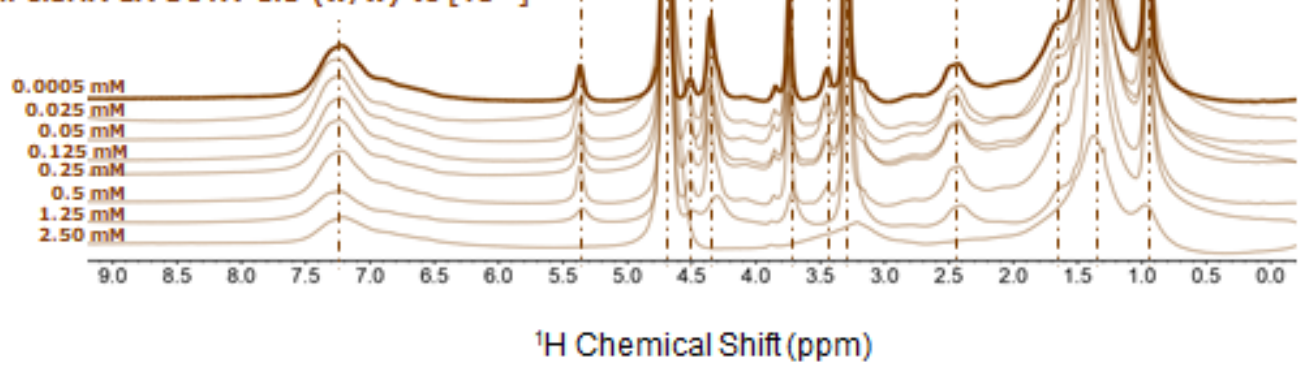

Figure S6. NMR spectra of SMA-EA-DOTA in the presence of paramagnetic metals. Stacking of $1 \mathrm{D}{ }^{1} \mathrm{H}-\mathrm{NMR}$ of DMPC:SMA-EA-DOTA 1:1 (w/w) macro-nanodiscs in the presence of different $\mathrm{Ln}^{3+}$ ions. Lanthanide trivalent ions such as $\mathrm{Eu}^{3+}, \mathrm{Gd}^{3+}, \mathrm{Dy}^{3+}, \mathrm{Er}^{3+}, \mathrm{Yb}^{3+}$ are respectively shown in a), b), c), d), and e). 

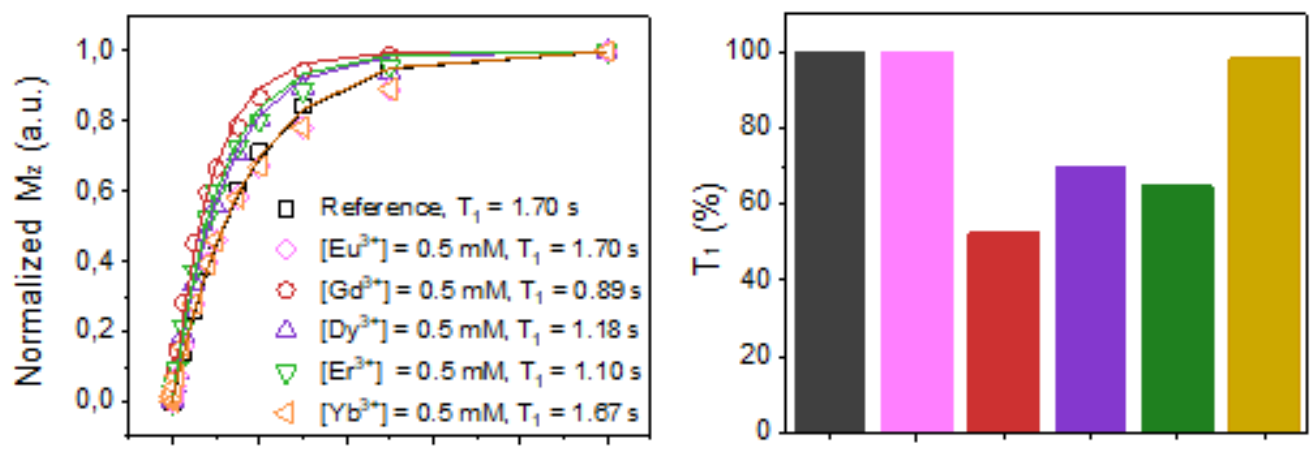

Aromatic
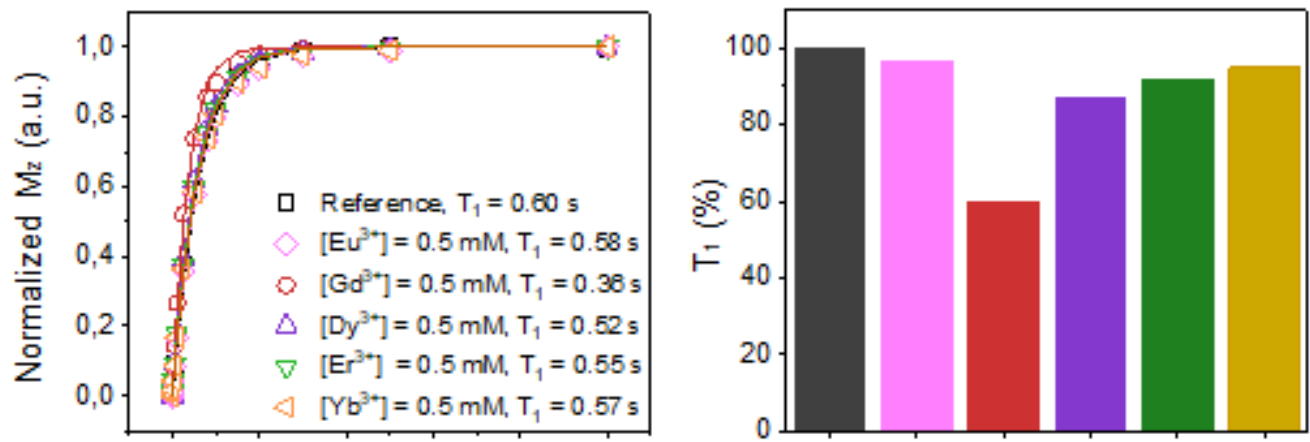

$\gamma$
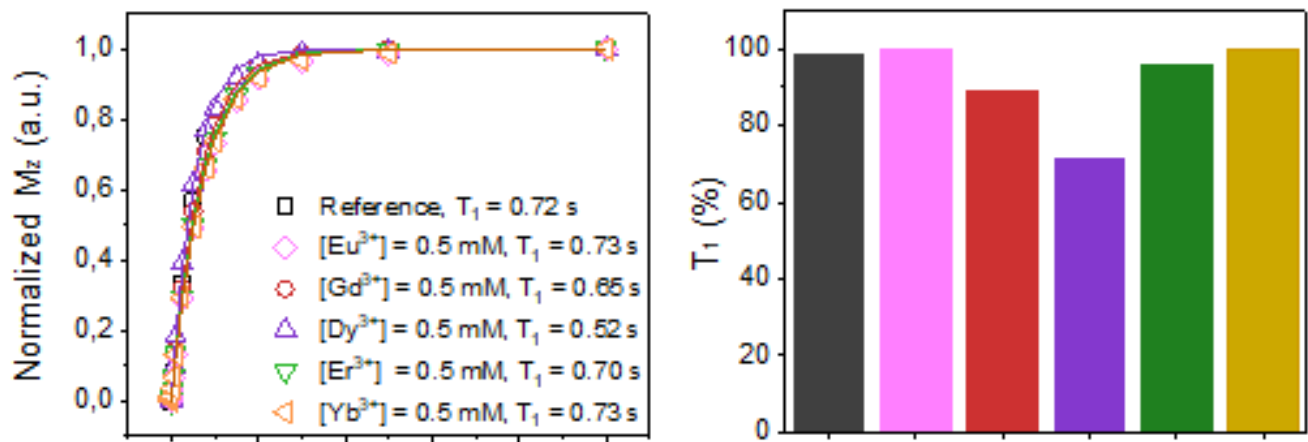

4-13
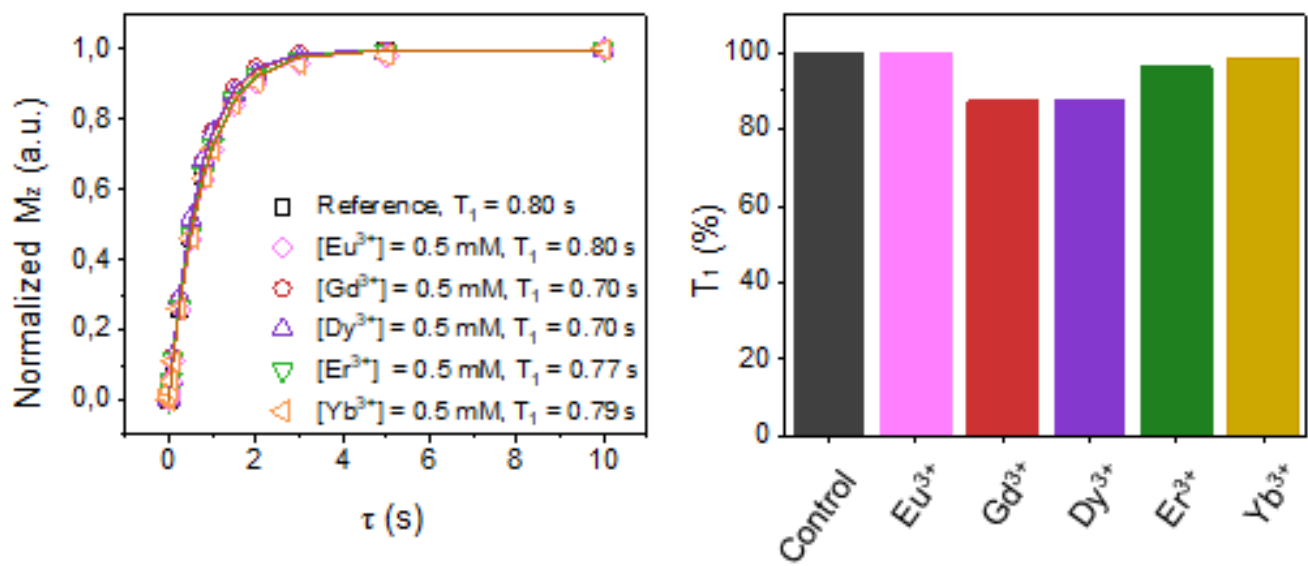

Figure S7. Normalized fitting (left panel) and normalized $T_{1}$ values (right panel) for DMPC: SMA-EA-DOTA 1:1 (w/w) macro-nanodises in the presence of $0.5 \mathbf{M ~ L n}^{3+}$. From top to bottom data relative to the aromatic, gamma, 2-13, and $14{ }^{1} \mathrm{H}$ peaks 